\title{
Interpolating 't Hooft model between instant and front forms
}

\author{
Bailing Ma๑ and Chueng-Ryong Ji® \\ Department of Physics, North Carolina State University, Raleigh, North Carolina 27695-8202, USA
}

(Received 21 May 2021; accepted 15 June 2021; published 16 August 2021)

The 't Hooft model, i.e., the two-dimensional quantum chromodynamics in the limit of infinite number of colors, is interpolated by an angle parameter $\delta$ between $\delta=0$ for the instant form dynamics (IFD) and $\delta=\pi / 4$ for the light-front dynamics (LFD). With this parameter $\delta$, we formulate the interpolating mass gap equation which takes into account the nontrivial vacuum effect on the bare fermion mass to find the dressed fermion mass. Our interpolating mass gap solutions not only reproduce the previous IFD result at $\delta=0$ as well as the previous LFD result at $\delta=\pi / 4$ but also link them together between the IFD and LFD results with the $\delta$ parameter. We find the interpolation angle independent characteristic energy function which satisfies the energy-momentum dispersion relation of the dressed fermion, identifying the renormalized fermion mass function and the wave function renormalization factor. The renormalized fermion condensate is also found independent of $\delta$, indicating the persistence of the nontrivial vacuum structure even in the LFD. Using the dressed fermion propagator interpolating between IFD and LFD, we derive the corresponding quark-antiquark bound-state equation in the interpolating formulation verifying its agreement with the previous bound-state equations in the IFD and LFD at $\delta=0$ and $\delta=\pi / 4$, respectively. The mass spectra of mesons bearing the feature of the Regge trajectories are found independent of the $\delta$-parameter reproducing the previous results in LFD and IFD for the equal mass quark and antiquark bound states. The Gell-Mann-Oakes-Renner relation for the pionic ground-state in the zero fermion mass limit is confirmed indicating that the spontaneous breaking of the chiral symmetry occurs in the 't Hooft model regardless of the quantization for $0 \leq \delta \leq \pi / 4$. We obtain the corresponding bound-state wave functions and discuss their reference frame dependence with respect to the frame independent LFD result. Applying them for the computation of the so-called quasi-parton distribution functions now in the interpolating formulation between IFD and LFD, we note a possibility of utilizing not only the reference frame dependence but also the interpolation angle dependence to get an alternative effective approach to the LFDlike results.

DOI: 10.1103/PhysRevD.104.036004

\section{INTRODUCTION}

The two-dimensional quantum chromodynamics $\left(\mathrm{QCD}_{2}\right)$ with the number of colors $N_{c} \rightarrow \infty$ has served as a theoretical laboratory for the study of strong interactions. In 't Hooft's seminal paper in 1974 [1], the power of $1 / N_{c}$ expansion [2] was demonstrated in solving $\mathrm{QCD}_{2}$ in the limit of $N_{c} \rightarrow \infty$, which was then widely studied also in relation to the string model and dual theories with the idea of $1 / N_{c}$ expansion as a topological expansion in the motion of physical strings (e.g., by Witten [3]). Under the large $N_{c}$ approximation, nonplanar diagrams are negligible and thus, for example, only the rainbow diagrams need to be summed over for the computation of the

Published by the American Physical Society under the terms of the Creative Commons Attribution 4.0 International license. Further distribution of this work must maintain attribution to the author(s) and the published article's title, journal citation, and DOI. Funded by SCOAP ${ }^{3}$. quark's self-mass. The two other parameters in $\mathrm{QCD}_{2}$ besides $N_{c}$, are the dimensionful coupling constant $g$ and the quark mass $m$. Sharing the same mass dimension, $g$ and $m$ play an important role in determining the phase of $\mathrm{QCD}_{2}[4]$. Depending on the value of the dimensionless coupling $g^{2} N_{c} / m^{2}$, it is known that there are at least two phases in $\mathrm{QCD}_{2}$ [5]. While the regime of the strong coupling phase which does not require the finiteness condition on the dimensionless coupling $g^{2} N_{c} / m^{2}$ [6] can be studied by the bosonization method [7], the regime of the weak coupling phase which keeps the so-called "'t Hooft coupling" $\lambda \sim$ $g^{2} N_{c}$ finite in the limit of not only $N_{c} \rightarrow \infty$ but also $g \rightarrow 0$ is investigated typically in $\mathrm{QCD}_{2}$. Although the strong coupling regime of $\mathrm{QCD}_{2}$ is interesting and deserves further study, the scope of the present work is limited to the weak coupling regime of $\mathrm{QCD}_{2}$. Yet, we notice that solving $\mathrm{QCD}_{2}$ in the weak coupling regime, i.e., 't Hooft model, is still highly nontrivial as the theory captures the property of quark confinement and involves the infrared-cutoff procedures discussed in two-dimensional gauge field theories $[8,9]$. 
In particular, we notice that the 't Hooft model was originally formulated and solved in the light front dynamics (LFD) [1] well before it was rederived and discussed in the instant form dynamics (IFD) [10]. The numerical solution of the 't Hooft model in the IFD was presented in the rest frame of the meson [11] and more recently also in the moving frames [12]. While a particular family of the axial gauges interpolated between the IFD and the LFD was explored, the principal-value prescription for regulating the infrared divergences was shown to be inconsistent with the interpolated general axial gauges [9]. Since then, however, this issue involving the interpolation between the IFD and the LFD has not yet been examined any further although the nontrivial vacua in two-dimensional models including $\mathrm{QED}_{2}$ have been extensively discussed [13]. It thus motivates us to explore the interpolation of the 't Hooft model between the IFD and the LFD fully beyond the gauge sector and discuss the outcome of the full interpolation which naturally remedies the previous issue on novel inconsistency with the interpolating gauge [9].

Before we get into the details and specific discussions on the $1+1$ dimensional nature of the 't Hooft model, we first briefly summarize the general remarks on distinguished features of the IFD and the LFD proposed originally by Dirac in 1949 [14] and the efforts of interpolating them together [13,15-21]. The LFD has the advantage of having the maximum number (seven) of the kinematic operators among the ten Poincaré operators. More kinematic operators provide more symmetries that effectively save the efforts of solving dynamic equations. The conversion of the dynamic operator in one form of the dynamics into the kinematic operator in another form of the dynamics can be traced by introducing an interpolation angle parameter spanning between the two different forms of the dynamics. This in fact motivates the study of the interpolation between the IFD and the LFD.

In our previous works, we have applied the interpolation method to the scattering amplitude of two scalar particles [17], the electromagnetic gauge fields [18], as well as the helicity spinors [19] and established the interpolating QED theory between the IFD and LFD [15]. In particular, we presented [15] the formal derivation of the interpolating QED in the canonical field theory approach and discussed the constraint fermion degrees of freedom, which appear uniquely in the LFD. The constraint component of the fermion degrees of freedom in LFD results in the instantaneous contribution to the fermion propagator, which is genuinely distinguished from the ordinary equal-time forward and backward propagation of the relativistic fermion degrees of freedom. The helicity of the onmass-shell fermion spinors in LFD is also distinguished from the ordinary Jacob-Wick helicity in the IFD with respect to whether the helicity depends on the reference frame or not. Our analyses clarified any conceivable confusion in the prevailing notion of the equivalence between the infinite momentum frame (IMF) approach and the LFD.

To link the $1+1$ dimensional IFD space-time coordinates with the LFD ones, we introduce the "hat notation" for the interpolating variables, as we have done in Refs. [15,17-19]:

$$
\left(\begin{array}{l}
x^{\hat{+}} \\
x^{\hat{*}}
\end{array}\right)=\left(\begin{array}{cc}
\cos \delta & \sin \delta \\
\sin \delta & -\cos \delta
\end{array}\right)\left(\begin{array}{l}
x^{0} \\
x^{1}
\end{array}\right) \text {, }
$$

where the interpolation angle $\delta$ is allowed to be in the region of $0 \leq \delta \leq \frac{\pi}{4}$. When $\delta=0$, we recover the IFD coordinates $\left(x^{0},-x^{1}\right)$, and when $\delta=\pi / 4$, we arrive at the LFD coordinates denoted typically by $x^{ \pm}=\left(x^{0} \pm x^{1}\right) / \sqrt{2}$ without the "hat."

In this coordinate system, the metric becomes

$$
g^{\hat{\mu} \hat{\nu}}=g_{\hat{\mu} \hat{\nu}}=\left(\begin{array}{cc}
\mathbb{C} & \mathbb{S} \\
\mathbb{S} & -\mathbb{C}
\end{array}\right),
$$

where we use the short-hand notation $\mathbb{C}=\cos 2 \delta$ and $\mathbb{S}=\sin 2 \delta$. Apparently, the interpolating $g^{\hat{\mu} \hat{\nu}}$ goes to the IFD metric $\left(\begin{array}{ll}1 & 0 \\ 0 & -1\end{array}\right)$ when $\delta=0$, and the LFD metric $\left(\begin{array}{ll}0 & 1 \\ 1 & 0\end{array}\right)$ when $\delta=\pi / 4$.

The components of covariant and contravariant twovector $a$ are then related with each other by

$$
\begin{array}{ll}
a_{\hat{+}}=\mathbb{C} a^{\hat{+}}+\mathbb{S} a^{\hat{\imath}} ; & a^{\hat{+}}=\mathbb{C} a_{\hat{+}}+\mathbb{S} a_{\hat{\iota}} ; \\
a_{\hat{\iota}}=\mathbb{S} a^{\hat{+}}-\mathbb{C} a^{\hat{\epsilon}} ; & a^{\hat{\imath}}=\mathbb{S} a_{\hat{+}}-\mathbb{C} a_{\hat{\varkappa}},
\end{array}
$$

and the inner product of two vectors $a$ and $b$ can be written as

$$
a^{\hat{\mu}} b_{\hat{\mu}}=\mathbb{C}\left(a^{\hat{+}} b^{\hat{+}}-a^{\hat{*}} b^{\hat{\varkappa}}\right)+\mathbb{S}\left(a^{\hat{+}} b^{\hat{-}}+a^{\hat{*}} b^{\hat{+}}\right) .
$$

The same transformation as shown in Eq. (1) applies to momentum variables as well, i.e.,

$$
\begin{aligned}
& p^{\hat{+}}=p^{0} \cos \delta+p^{1} \sin \delta \\
& p^{\hat{\varkappa}}=p^{0} \sin \delta-p^{1} \cos \delta .
\end{aligned}
$$

According to Eq. (3), we also have

$$
\begin{aligned}
& p_{\hat{+}}=p^{0} \cos \delta-p^{1} \sin \delta ; \\
& p_{\text {^ }}=p^{0} \sin \delta+p^{1} \cos \delta .
\end{aligned}
$$

A useful relationship for the energy-momentum of the onmass-shell particle with mass $m$ and two-momentum vector $p^{\hat{\mu}}$ can be found as follows 


$$
\left(p^{\hat{+}}\right)^{2}=\left(p_{\hat{\sim}}\right)^{2}+\mathbb{C} m^{2} .
$$

With the "hat notation," the theory of $\mathrm{QCD}_{1+1}$ in the interpolating quantization is then given by the Lagrangian density $^{1}$

$$
\mathcal{L}=-\frac{1}{4} F_{\hat{\mu} \hat{\nu}}^{a} F^{\hat{\mu} \hat{\nu} a}+\bar{\psi}\left(i \gamma^{\hat{\mu}} D_{\hat{\mu}}-m\right) \psi
$$

where

$$
D_{\hat{\mu}}=\partial_{\hat{\mu}}-i g A_{\hat{\mu}}^{a} t_{a}
$$

and

$$
F_{\hat{\mu} \hat{\nu}}^{a}=\partial_{\hat{\mu}} A_{\hat{\nu}}^{a}-\partial_{\hat{\nu}} A_{\hat{\mu}}^{a}+g f^{a b c} A_{\hat{\mu}}^{b} A_{\hat{\nu}}^{c} .
$$

In this work, we start from the interpolating Lagrangian density, Eq. (9), derive the corresponding Hamiltonian and solve the mass gap equation which interpolates between the IFD and the LFD. We then apply the solutions of mass gap equation to the calculations of chiral condensates and the quark-antiquark bound states to find the meson mass spectra and the corresponding wave functions. As expected for any physical observables, the meson mass spectra are found to be independent of the interpolation angle parameter. Since we obtain the meson wave functions in terms of the interpolation angle parameter $\delta$, we use these $\delta$ dependent wave functions to compute the corresponding parton distribution functions (PDFs), comparing them with the PDFs in the LFD and the so-called quasi-PDFs based on the IMF approach in IFD [22].

The paper is organized as follows. In Sec. II, we derive the fermion mass gap equation in $\mathrm{QCD}_{1+1}\left(N_{c} \rightarrow \infty\right)$ in the quantization interpolating between the IFD and the LFD, using a couple of different methods, namely, the Hamiltonian method and the Feynman-diagram method. In Sec. III, we present the solutions of the mass gap equation numerically spanning the interpolation angle between $\delta=0$ (IFD) and $\delta=\pi / 4$ (LFD). In Sec. IV, we apply the mass gap solutions to the calculations of the chiral condensates and the constituent quark mass defined in the full fermion propagator. In Sec. V, we derive the quark-antiquark bound-state equations in the interpolating dynamics and present their solutions in Sec. VI, including the meson mass spectra, wave functions, and (quasi-)PDFs in Secs. VI A, VIB and VIC, respectively. The summary

\footnotetext{
${ }^{1}$ It is worth noting that our definition of $g$ is the same with that of Ref. [10], but differs with that of Ref. [1] by a factor of $\frac{1}{\sqrt{2}}$, i.e., when $\delta \rightarrow \frac{\pi}{4}$, (quantities with a superscript " $\mathrm{t}$ " denote the notation used in Ref. [1], and the ones without are ours)
}

$$
A_{\mu}^{\mathrm{t}}=-i \sqrt{2} A_{\mu}^{a} t_{a}, \quad g^{\mathrm{t}}=\frac{1}{\sqrt{2}} g, \quad \text { and } \quad \gamma_{\mu}^{\mathrm{t}}=-i \gamma^{\mu} .
$$

and conclusions follow in Sec. VII. In Appendix A, we describe in detail the derivation of the interacting quark/ antiquark spinor representation using the Bogoliubov transformation. In Appendix B, we show the method of minimizing the vacuum energy with respect to the Bogoliubov angle in getting the mass gap equation. In Appendix $\mathrm{C}$, we discuss the interpolating mass gap equation and solution in terms of the rescaled variables with respect to the mass dimension $\sqrt{2 \lambda}$ and its treatment associated with the $\lambda=0$ (Free) case vs the $\lambda \neq 0$ (Interacting) case. In Appendix D, we present additional numerical solutions of the mesonic wave function for a few different quark masses beyond the ones presented in Sec. VIB. The corresponding quasi-PDFs are discussed in Appendix E. In Appendix F, we present the quarkantiquark bound-state equations and solutions in the rest frame of the meson.

\section{THE MASS GAP EQUATION}

In this section, we will derive the quark self-energy equation in $\mathrm{QCD}_{1+1}\left(N_{c} \rightarrow \infty\right)$ in the interpolating dynamics between the IFD and the LFD. While we use two different methods, i.e., the Hamiltonian method in Sec. II A and the Feynman-diagram method in Sec. II B, we show that both methods provide exactly the same set of equations. When $\delta \rightarrow 0, \mathbb{C} \rightarrow 1$ and $p_{\hat{\imath}} \rightarrow p^{1}$, these equations become the IFD mass gap equations presented in Ref. [10] (i.e., Eqs. (3.18) and (3.19) of Ref [10]). The agreement to Ref. [1] of the $\delta \rightarrow \frac{\pi}{4}$ limit is discussed in Sec. II C.

\section{A. The Hamiltonian method}

Before we start, we need to choose a gauge as in the case of any gauge field theory. We adopt here the interpolating axial gauge, i.e., $A_{\wedge}^{a}=0$, as explored previously in Ref. [9]. In this gauge, the gluon self-couplings are absent. With the gauge condition, Eq. (9) reduces to

$$
\mathcal{L}=\frac{1}{2}\left(\partial_{\hat{\imath}} A_{\hat{+}}^{a}\right)^{2}+\bar{\psi}\left(i \gamma^{\hat{+}} D_{\hat{+}}+i \gamma^{\hat{\prime}} \partial_{\hat{-}}-m\right) \psi .
$$

As no interpolation-time derivative of $A_{\hat{+}}^{a}$, i.e., $\partial_{\hat{+}} A_{\hat{+}}^{a}$, appears in Eq. (12), $A_{\hat{+}}^{a}$ is not a dynamical variable but a constrained degree of freedom. We substitute this constrained degree of freedom using the equation of motion for the gluon field $A_{\hat{+}}^{a}$ given by

$$
\partial_{\hat{\imath}}^{2} A_{\hat{+}}^{a}=\psi^{\dagger} \gamma^{0} \gamma^{\hat{+}} g t^{a} \psi \equiv \rho^{a}=J^{\hat{+} a} .
$$

The general solution of Eq. (13) is given by

$$
\begin{aligned}
& A_{\hat{+}}^{a}\left(x^{\hat{+}}, x^{\hat{*}}\right) \\
& =\frac{1}{2} \int d y^{\hat{\imath}}\left|x^{\hat{\varkappa}}-y^{\hat{\imath}}\right| \rho^{a}\left(x^{\hat{+}}, y^{\hat{\varkappa}}\right)-x^{\hat{\varkappa}} F^{a}\left(x^{\hat{+}}\right)+B^{a}\left(x^{\hat{+}}\right),
\end{aligned}
$$


where $F^{a}$ and $B^{a}$ are constant. While $B^{a}$ is irrelevant as it can always be eliminated by a gauge transformation, $F^{a}$ is a background electric field which can provide some interesting physical effect such as the axial anomaly in Abelian gauge field theory [23]. For the color-singlet sector in the non-Abelian gauge field theory, however, the background field $F^{a}$ has no effect, e.g., on the spectrum of hadrons in the $q \bar{q}$ channel. For this reason, we drop the background field $F^{a}$ and take the first term of Eq. (14) as the solution of $A_{\hat{+}}^{a}\left(x^{\hat{+}}, x^{\hat{\mathcal{A}}}\right)$ in this work. More details of the discussion on the effect from dropping the background field in the 't Hooft model can be found in Ref. [10].

The energy-momentum tensor in the interpolation form is

$$
T^{\hat{\mu}}{ }_{\hat{\nu}}=-F^{\hat{\mu} \hat{\lambda} a} F_{\hat{\nu} \hat{\lambda}}^{a}+i \bar{\psi} \gamma \gamma^{\hat{\mu}} D_{\hat{\nu}} \psi-g^{\hat{\mu}} \hat{\nu} \mathcal{L} .
$$

Thus, the interpolating Hamiltonian is

$$
\begin{aligned}
& H \equiv P_{\hat{+}}=\int d x^{\hat{\imath}} T_{\hat{+}}^{\hat{+}}=\int d x^{\hat{\imath}}\left(\frac{1}{2}\left(\partial_{\hat{\imath}} A_{\hat{+}}^{a}\right)^{2}\right. \\
& \left.+\psi^{\dagger}\left(x^{\hat{-}}\right)\left(-i \gamma^{0} \gamma^{\hat{-}} \partial_{\hat{-}}+\gamma^{0} m\right) \psi\left(x^{\hat{*}}\right)\right) \text {. }
\end{aligned}
$$

As we have shown in [15], all components of the $\psi$ field are dynamical degrees of freedom for $0 \leq \delta<\pi / 4$, while half of the components become constrained for $\delta=\pi / 4$. The field operator conjugate to $\psi(x)$ is

$$
\Pi(x)=\frac{\partial \mathcal{L}}{\partial\left(\partial_{\hat{+}} \psi(x)\right)}=i \gamma^{0} \gamma^{\hat{+}} \psi^{\dagger}(x) .
$$

The anticommutation relation at $x^{\hat{+}}=x^{\prime \hat{+}}$ is

$$
\begin{aligned}
& \left\{\Pi(x), \psi\left(x^{\prime}\right)\right\}_{x^{\hat{+}}=x^{\prime \hat{\dagger}}}=i \gamma^{0} \gamma^{\hat{+}}\left\{\psi^{\dagger}\left(x^{\hat{-}}\right), \psi\left(x^{\prime \hat{A}}\right)\right\} \\
& =i \delta\left(x^{\hat{\imath}}-x^{\prime \hat{\wedge}}\right) \text {. }
\end{aligned}
$$

Consequently,

$$
\left\{\psi^{\dagger}\left(x^{\hat{\imath}}\right), \psi\left(x^{\prime \hat{\imath}}\right)\right\}=\left(\gamma^{0} \gamma^{\hat{+}}\right)^{-1} \delta\left(x^{\hat{\imath}}-x^{\prime \hat{\imath}}\right) .
$$

The Dirac field $\psi$ can be expanded in terms of the quark creation and annihilation operators

$$
\begin{aligned}
& \psi\left(x^{\hat{\imath}}\right)=\int \frac{d p_{\hat{\iota}}}{2 \pi \sqrt{2 p^{\hat{+}}}} \\
& \times\left[b\left(p_{\hat{\iota}}\right) u\left(p_{\hat{\iota}}\right)+d^{\dagger}\left(-p_{\hat{\iota}}\right) v\left(-p_{\hat{\iota}}\right)\right] \mathrm{e}^{-i p_{\hat{\iota}} x^{\underline{\underline{ }}}} .
\end{aligned}
$$

When the form goes to the limit of IFD, i.e., $\delta \rightarrow 0$, $p_{\curlywedge} \rightarrow-p_{1}=p^{1}$, and $x^{\wedge} \rightarrow-x^{1}$, and Eq. (20) becomes the ordinary field operator expansion in IFD. ${ }^{2}$

\footnotetext{
${ }^{2}$ It differs from the expression in Ref. [10] by a normalization factor $\frac{1}{\sqrt{2 p^{\dagger}}}$, which was inserted in order to be consistent with standard textbook [24].
}

The nontrivial vacuum, $\mid \Omega>$, which is defined by

$$
b^{i}\left|\Omega>=0, \quad d^{i}\right| \Omega>=0,
$$

is different from the trivial vacuum $\mid 0>$ defined by

$$
b^{i(0)}\left|0>=0, \quad d^{i(0)}\right| 0>=0 .
$$

The trivial and nontrivial sets of creation and annihilation operators are related by a Bogoliubov transformation

$$
\begin{aligned}
\left(\begin{array}{c}
b^{i}\left(p_{\hat{\lrcorner}}\right) \\
d^{i \dagger}\left(-p_{\hat{\iota}}\right)
\end{array}\right)= & \left(\begin{array}{cc}
\cos \zeta\left(p_{\hat{\iota}}\right) & -\sin \zeta\left(p_{\hat{\lrcorner}}\right) \\
\sin \zeta\left(p_{\hat{\iota}}\right) & \cos \zeta\left(p_{\hat{\iota}}\right)
\end{array}\right) \\
& \cdot\left(\begin{array}{c}
b^{i(0)}\left(p_{\hat{\iota}}\right) \\
d^{i(0) \dagger}\left(-p_{\hat{\iota}}\right)
\end{array}\right) .
\end{aligned}
$$

The nontrivial set of operators, just like the trivial ones, satisfy the canonical anticommutation relations at $x^{\hat{+}}=x^{\prime \hat{+}}$

$$
\begin{aligned}
& \left\{b^{i}\left(p_{\hat{\iota}}, x^{\hat{+}}\right), b^{\dagger j}\left(p_{\hat{\iota}}^{\prime}, x^{\prime \hat{+}}\right)\right\}_{x^{\hat{+}}=x^{\prime \hat{+}}}=2 \pi \delta\left(p_{\hat{\iota}}-p_{\hat{\lrcorner}}^{\prime}\right) \delta^{i j}, \\
& \left\{d^{i}\left(-p_{\hat{\iota}}, x^{\hat{+}}\right), d^{\dagger j}\left(-p_{\hat{\iota}}^{\prime}, x^{\prime \hat{+}}\right)\right\}_{x^{\hat{+}}=x^{\prime \hat{+}}}=2 \pi \delta\left(p_{\hat{\iota}}-p_{\hat{\iota}}^{\prime}\right) \delta^{i j},
\end{aligned}
$$

and all others are zero.

The spinors can be defined through a combination of boost and Bogoliubov transformation, which can be represented by

$$
\theta\left(p_{\hat{\lrcorner}}\right)=\theta_{f}\left(p_{\hat{\iota}}\right)+2 \zeta\left(p_{\hat{\iota}}\right),
$$

where $\theta_{f}\left(p_{\lrcorner}\right)$is the boost part given by

$$
\theta_{f}\left(p_{\hat{\sim}}\right)=\arctan \frac{p_{\hat{\varkappa}}}{\sqrt{\mathbb{C}} m},
$$

and $\zeta\left(p_{\hat{\iota}}\right)$ is the Bogoliubov angle defined in Eq. (23).

While the details of the derivation are given in Appendix A, the results of the spinors are given by

$$
u\left(p_{\hat{\lrcorner}}\right)=\sqrt{2 p^{\hat{+}}}\left(\begin{array}{c}
\sqrt{\frac{1-\sin \theta\left(p_{\lrcorner}\right)}{2(\cos \delta-\sin \delta)}} \\
\sqrt{\frac{1+\sin \theta\left(p_{\lrcorner}\right)}{2(\cos \delta+\sin \delta)}}
\end{array}\right)
$$

and

$$
v\left(-p_{\hat{\prime}}\right)=\sqrt{2 p^{\hat{+}}}\left(\begin{array}{c}
\sqrt{\frac{1+\sin \theta\left(p_{\dot{\Delta}}\right)}{2(\cos \delta-\sin \delta)}} \\
-\sqrt{\frac{1-\sin \theta\left(p_{\dot{\Delta}}\right)}{2(\cos \delta+\sin \delta)}}
\end{array}\right) .
$$

In the case of free particles, Eqs. (28) and (29) get simplified with $\theta\left(p_{\hat{\varkappa}}\right)=\theta_{f}\left(p_{\hat{\iota}}\right)$ given by Eq. (27) as 


$$
u^{(0)}\left(p_{\hat{\varkappa}}\right)=\left(\begin{array}{c}
\sqrt{\frac{p^{\hat{f}}-p_{\hat{\iota}}}{\cos \delta-\sin \delta}} \\
\sqrt{\frac{p^{\hat{f}}+p_{\hat{\prime}}}{\cos \delta+\sin \delta}}
\end{array}\right)
$$

and

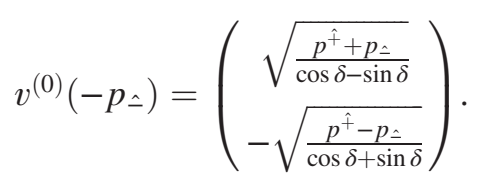

Now, plugging the gluon field solution Eq. (14) without the background field into Eq. (16), we obtain the interpolating Hamiltonian as

$$
\begin{aligned}
& H=\int d x^{\hat{\imath}} \psi^{\dagger}\left(x^{\hat{-}}\right)\left(-i \gamma^{0} \gamma^{\hat{\imath}} \partial_{\hat{\imath}}+\gamma^{0} m\right) \psi\left(x^{\hat{\varkappa}}\right) \\
& -\frac{1}{4} \int d x^{\hat{\imath}} \int d y^{\hat{\imath}} \rho^{a}\left(x^{\hat{\imath}}\right)\left|x^{\hat{\imath}}-y^{\hat{\imath}}\right| \rho^{a}\left(y^{\hat{\varkappa}}\right) \\
& =T+V \text {, }
\end{aligned}
$$

where the kinetic energy

$$
T=\int d x^{\hat{\imath}} \psi^{\dagger}\left(x^{\hat{\imath}}\right)\left(-i \gamma^{0} \gamma^{\hat{\imath}} \partial_{\hat{-}}+\gamma^{0} m\right) \psi\left(x^{\hat{\imath}}\right),
$$

and the potential energy

$$
\begin{aligned}
& V=-\frac{1}{4} \int d x^{\hat{\imath}} \int d y^{\hat{\imath}} \rho^{a}\left(x^{\hat{\imath}}\right)\left|x^{\hat{\imath}}-y^{\hat{\imath}}\right| \rho^{a}\left(y^{\hat{\sim}}\right) \\
& =-\frac{g^{2}}{4} \int d x^{\hat{\imath}} \int d y^{\hat{\imath}}\left|x^{\hat{\imath}}-y^{\hat{\imath}}\right| \psi^{\dagger}\left(x^{\hat{\imath}}\right) \gamma^{0} \gamma^{\hat{\dagger}} t^{a} \psi\left(x^{\hat{\imath}}\right) \\
& \times \psi^{\dagger}\left(y^{\hat{-}}\right) \gamma^{0} \gamma^{\hat{+}} t^{a} \psi\left(y^{\hat{A}}\right) \text {. }
\end{aligned}
$$

Now, if we define the 't Hooft coupling as

$$
\lambda=\frac{g^{2}\left(N_{c}-1 / N_{c}\right)}{4 \pi},
$$

then $\lambda$ has the dimension of mass squared in $1+1$ dimension. By normal-ordering the Hamiltonian, we can write it in three pieces

$$
H=L N_{c} \mathcal{E}_{v}+: H_{2}:+: H_{4}:
$$

Here, the vacuum energy density $\mathcal{E}_{v}$ for the onedimensional volume $L$ is given by

$$
\begin{aligned}
& \mathcal{E}_{v}=\int \frac{d p_{\hat{\varkappa}}}{(2 \pi)\left(2 p^{\hat{+}}\right)} \operatorname{Tr}\left[\left(-\gamma^{0} \gamma^{\hat{\underline{\prime}}} p_{\hat{\iota}}+m \gamma^{0}\right) v\left(-p_{\hat{\iota}}\right) v^{\dagger}\left(-p_{\hat{\iota}}\right)\right] \\
& +\frac{\lambda}{4 \pi} \int \frac{d p_{\hat{\epsilon}}}{2 p^{\hat{+}}} \int \frac{d k_{\hat{\varkappa}}}{2 k^{\hat{+}}} \frac{1}{\left(p_{\hat{\iota}}-k_{\hat{\iota}}\right)^{2}} \operatorname{Tr}\left[\gamma^{0} \gamma^{\hat{+}} u\left(k_{\hat{\varkappa}}\right) u^{\dagger}\left(k_{\hat{\varkappa}}\right) \gamma^{0} \gamma^{\hat{+}} v\left(-p_{\hat{\iota}}\right) v^{\dagger}\left(-p_{\hat{\varkappa}}\right)\right] \text {, }
\end{aligned}
$$

the two-body interaction term including the kinetic energy is given by

$$
: H_{2}:=: T:+: V_{2}:
$$

with

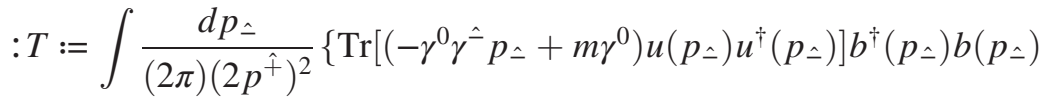

$$
\begin{aligned}
& +\operatorname{Tr}\left[\left(-\gamma^{0} \gamma^{\hat{\prime}} p_{\hat{\iota}}+m \gamma^{0}\right) v\left(-p_{\hat{\iota}}\right) u^{\dagger}\left(p_{\hat{\iota}}\right)\right] b^{\dagger}\left(p_{\hat{\varkappa}}\right) d^{\dagger}\left(-p_{\hat{\varkappa}}\right) \\
& +\operatorname{Tr}\left[\left(-\gamma^{0} \gamma^{\hat{\epsilon}} p_{\hat{\iota}}+m \gamma^{0}\right) u\left(p_{\hat{\iota}}\right) v^{\dagger}\left(-p_{\hat{\iota}}\right)\right] d\left(-p_{\hat{\iota}}\right) b\left(p_{\hat{\varkappa}}\right)
\end{aligned}
$$

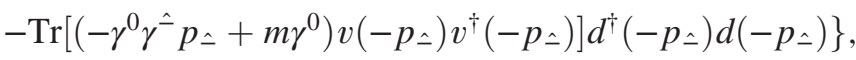

and

$$
\begin{aligned}
& : V_{2}:=\frac{\lambda}{2} \int \frac{d p_{\hat{\imath}}}{(2 \pi)\left(2 p^{\hat{+}}\right)^{2}} \int \frac{d k_{\varkappa}}{\left(2 k^{\hat{+}}\right)\left(p_{\iota}-k_{\varkappa}\right)^{2}}
\end{aligned}
$$

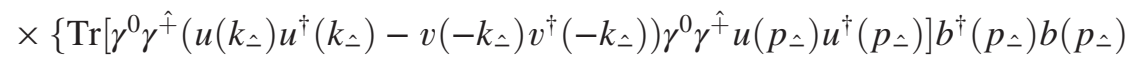

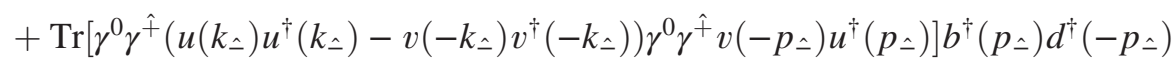

$$
\begin{aligned}
& +\operatorname{Tr}\left[\gamma^{0} \gamma^{\hat{+}}\left(u\left(k_{\hat{\varkappa}}\right) u^{\dagger}\left(k_{\hat{\varkappa}}\right)-v\left(-k_{\hat{\varkappa}}\right) v^{\dagger}\left(-k_{\hat{\varkappa}}\right)\right) \gamma^{0} \gamma^{\hat{+}} u\left(p_{\hat{\varkappa}}\right) v^{\dagger}\left(-p_{\hat{\iota}}\right)\right] d\left(-p_{\hat{\varkappa}}\right) b\left(p_{\hat{\varkappa}}\right) \\
& \left.-\operatorname{Tr}\left[\gamma^{0} \gamma^{\hat{+}}\left(u\left(k_{\hat{\varkappa}}\right) u^{\dagger}\left(k_{\hat{\varkappa}}\right)-v\left(-k_{\hat{\varkappa}}\right) v^{\dagger}\left(-k_{\hat{\varkappa}}\right)\right) \gamma^{0} \gamma^{\hat{+}} v\left(-p_{\hat{\varkappa}}\right) v^{\dagger}\left(-p_{\hat{\varkappa}}\right)\right] d^{\dagger}\left(-p_{\hat{\varkappa}}\right) d\left(-p_{\hat{\varkappa}}\right)\right\} \text {, }
\end{aligned}
$$


and the four-body interaction term is given by

$$
: H_{4}:=-\frac{g^{2}}{4} \int d x^{\hat{\imath}} \int d y^{\hat{\imath}}\left|x^{\hat{\imath}}-y^{\hat{\imath}}\right|: \psi^{\dagger}\left(x^{\hat{\imath}}\right) \gamma^{0} \gamma^{\hat{\dagger}} t^{a} \psi\left(x^{\hat{\imath}}\right) \psi^{\dagger}\left(y^{\hat{\imath}}\right) \gamma^{0} \gamma^{\hat{\dagger}} t^{a} \psi\left(y^{\hat{\imath}}\right): .
$$

Although the mass gap equation can be obtained either by minimizing $\mathcal{E}_{v}$ with respect to the Bogoliubov angle or by requiring $: \mathrm{H}_{2}$ : to be diagonal in the quark antiquark creation and annihilation operator basis, both methods provide the same resulting equations. While we present the derivation of minimizing $\mathcal{E}_{v}$ in Appendix B, we derive here the mass gap equations by requiring $: H_{2}:$ to be diagonal. The requirement of $: H_{2}:$ to be diagonal means that it must take the form

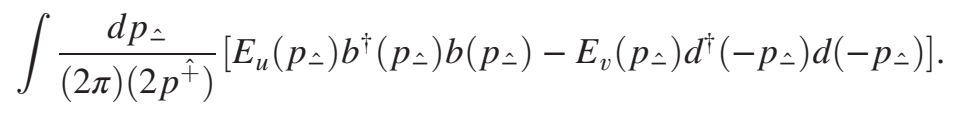

The divergent piece that comes out during the normal-ordering process is regulated removing the infinite energy [10], and using the principal value prescription as was done in Ref. [11],

$$
\int \frac{d y}{(x-y)^{2}} f(y) \rightarrow \int \frac{d y}{(x-y)^{2}}\left[f(y)-f(x)-(y-x) \frac{d f(x)}{d x}\right] \equiv f \frac{d y}{(x-y)^{2}} f(y) .
$$

Thus, the eigenvalue conditions on the spinors are given by

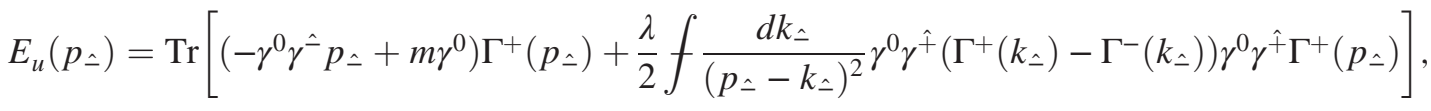

$$
\begin{aligned}
& E_{v}\left(p_{\hat{\iota}}\right)=\operatorname{Tr}\left[\left(-\gamma^{0} \gamma^{\hat{\imath}} p_{\hat{\iota}}+m \gamma^{0}\right) \Gamma^{-}\left(p_{\hat{\iota}}\right)+\frac{\lambda}{2} f \frac{d k_{\hat{\iota}}}{\left(p_{\hat{\iota}}-k_{\hat{\iota}}\right)^{2}} \gamma^{0} \gamma^{\hat{+}}\left(\Gamma^{+}\left(k_{\hat{\iota}}\right)-\Gamma^{-}\left(k_{\hat{\iota}}\right)\right) \gamma^{0} \gamma^{\hat{+}} \Gamma^{-}\left(p_{\hat{\iota}}\right)\right],
\end{aligned}
$$

where $\Gamma^{ \pm}$is defined by

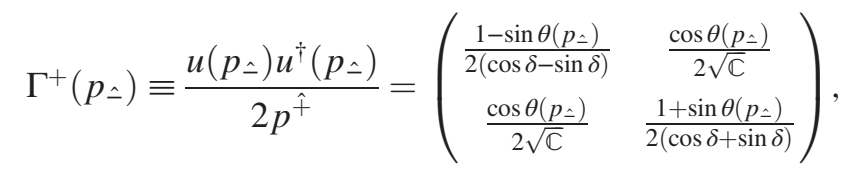

$$
\begin{aligned}
& \Gamma^{-}\left(p_{\lrcorner}\right) \equiv \frac{v\left(-p_{\hat{\lrcorner}}\right) v^{\dagger}\left(-p_{\hat{\lrcorner}}\right)}{2 p^{\hat{+}}}=\left(\begin{array}{cc}
\frac{1+\sin \theta\left(p_{\lrcorner}\right)}{2(\cos \delta-\sin \delta)} & -\frac{\cos \theta\left(p_{\lrcorner}\right)}{2 \sqrt{\complement}} \\
-\frac{\cos \theta\left(p_{\lrcorner}\right)}{2 \sqrt{\complement}} & \frac{1-\sin \theta\left(p_{\lrcorner}\right)}{2(\cos \delta+\sin \delta)}
\end{array}\right) .
\end{aligned}
$$

By using Eqs. (28) and (29), one may see that the matrices on the right-hand side of Eqs. (45) and (46) can be obtained by direct computation. Now, let us define

$$
E\left(p_{\hat{\lrcorner}}\right) \equiv \frac{\mathbb{C}}{2}\left[E_{u}\left(p_{\hat{\iota}}\right)-E_{v}\left(p_{\hat{\prime}}\right)\right]
$$

Then, by subtracting Eqs. (44a) and (44b) as well as plugging in Eqs. (45) and (46), we arrive at

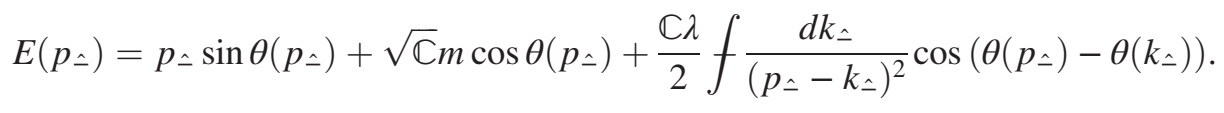

On the other hand, by adding them, we get

$$
E_{u}\left(p_{\curlywedge}\right)+E_{v}\left(p_{\lrcorner}\right)=-\frac{2 \mathbb{S}}{\mathbb{C}} p_{\lrcorner} .
$$

Also, we know that the off-diagonal elements of : $H_{2}:$ have to vanish 


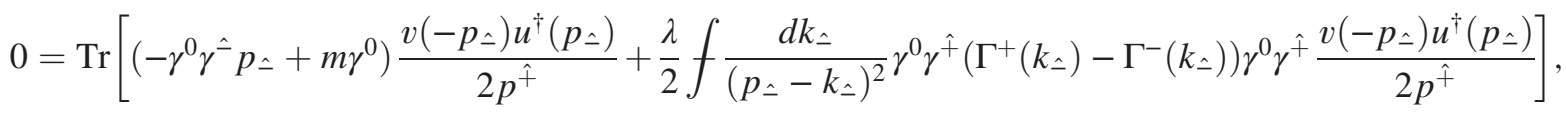

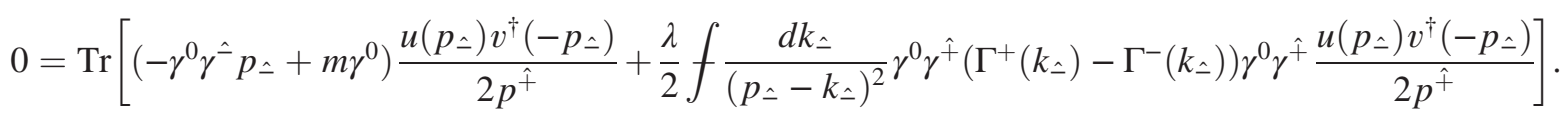

From either Eq. (50a) or Eq. (50b), we get

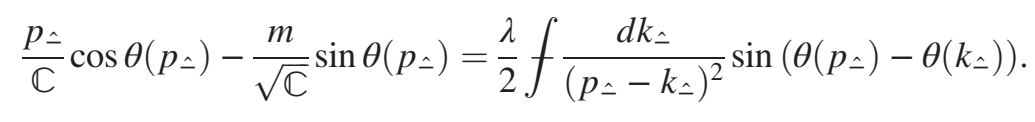

Equations (48) and (51) are the mass gap equations in the interpolating dynamics. The same set of mass gap equations can be derived using the Feynman diagram method as we present in the following Sec. II B.

\section{B. The Feynman diagram method}

The self-energy equation in the large $N_{c}$ approximation is drawn pictorially in Fig. 1. Following the Feynman rules for the gluon propagator, the free quark propagator and the vertex as $\frac{1}{k_{\perp}^{2}}, \frac{1}{\not-m+i \epsilon}$, and $g \gamma^{\hat{+}} t^{a}$, respectively, with the momentum assignment shown in Fig. 1, we have

$$
\Sigma\left(p_{\hat{\varkappa}}\right)=i \frac{\lambda}{2 \pi} f \frac{d k_{\llcorner} d k_{\hat{+}}}{\left(p_{\hat{-}}-k_{\hat{\varkappa}}\right)^{2}} \gamma^{\hat{+}} \frac{1}{\not k-m-\Sigma\left(k_{\hat{\varkappa}}\right)+i \epsilon} \gamma^{\hat{+}} .
$$

Writing the self-energy as

$$
\begin{aligned}
& \Sigma\left(p_{\hat{\iota}}\right)=\sqrt{\mathbb{C}} A\left(p_{\hat{\iota}}\right)+\gamma_{\hat{\iota}} B\left(p_{\hat{\iota}}\right) \\
& =\sqrt{\mathbb{C}} A\left(p_{\hat{\varkappa}}\right)+\left(\mathbb{S} \gamma^{\hat{+}}-\mathbb{C} \gamma^{\hat{\imath}}\right) B\left(p_{\hat{\iota}}\right),
\end{aligned}
$$

we express the dressed quark propagator as

$$
\begin{aligned}
& S(k)=\left[\not k-m-\Sigma\left(k_{\hat{\lrcorner}}\right)+i \epsilon\right]^{-1} \\
& =\left[\gamma^{\hat{+}}\left(k_{\hat{+}}-\mathbb{S} B\left(k_{\hat{\varkappa}}\right)\right)+\gamma^{\hat{\imath}}\left(k_{\hat{\iota}}+\mathbb{C} B\left(k_{\hat{\iota}}\right)\right)-\left(m+\sqrt{\mathbb{C}} A\left(k_{\varkappa}\right)\right)+i \epsilon\right]^{-1} .
\end{aligned}
$$

This dressed quark propagator can be obtained from the bare quark propagator with the replacement given by

$$
\left\{\begin{array}{l}
k_{\hat{+}} \rightarrow k_{\hat{+}}-\mathbb{S} B\left(k_{\hat{\varkappa}}\right) \\
k_{\hat{\lrcorner}} \rightarrow k_{\hat{\iota}}+\mathbb{C} B\left(k_{\hat{\varkappa}}\right) . \\
m \rightarrow m+\sqrt{\mathbb{C}} A\left(k_{\hat{\iota}}\right)
\end{array}\right.
$$

Then, Eq. (52) becomes

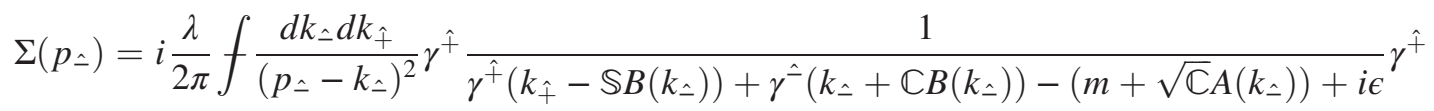

$$
\begin{aligned}
& =i \frac{\lambda}{2 \pi} f \frac{d k_{\varkappa} d k_{\hat{+}}}{\left(p_{\hat{\iota}}-k_{\hat{\iota}}\right)^{2}}
\end{aligned}
$$

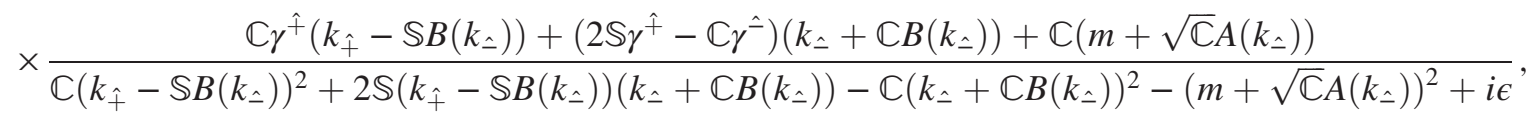

where we have used the algebra for the interpolating $\gamma$ matrices $\left(\gamma^{\hat{+}}\right)^{2}=\mathbb{C} \cdot \mathbf{I}_{2 \times 2}, \quad\left(\gamma^{\hat{A}}\right)^{2}=-\mathbb{C} \cdot \mathbf{I}_{2 \times 2}$, and $\left\{\gamma^{\hat{+}}, \gamma^{\hat{*}}\right\}=2 \mathbb{S} \cdot \mathbf{I}_{2 \times 2}$. Now, the two poles of $k_{\hat{+}}$ in the denominator of Eq. (56) are given by 


$$
-\frac{\mathbb{S}}{\mathbb{C}} k_{\hat{\varkappa}} \pm \sqrt{\left(\frac{k_{\hat{\epsilon}}}{\mathbb{C}}+B\left(k_{\hat{\varkappa}}\right)\right)^{2}+\left(\frac{m}{\sqrt{\mathbb{C}}}+A\left(k_{\hat{\varkappa}}\right)\right)^{2}} \mp i \epsilon^{\prime} .
$$

After doing the $k_{\hat{+}}$ pole integration using Cauchy's theorem, we get

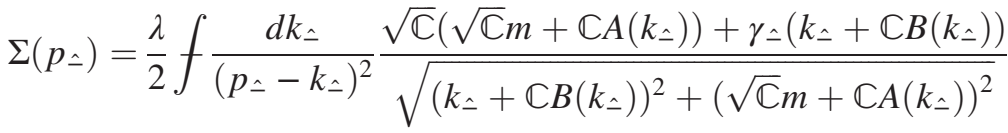

$$
\begin{aligned}
& =\frac{\lambda}{2} f \frac{d k_{\hat{\varkappa}}}{\left(p_{\hat{\iota}}-k_{\hat{\iota}}\right)^{2}}\left(\sqrt{\mathbb{C}} \cos \theta\left(k_{\hat{\varkappa}}\right)+\gamma_{\hat{\iota}} \sin \theta\left(k_{\hat{\iota}}\right)\right) \text {, }
\end{aligned}
$$

where $\theta\left(k_{\wedge}\right)$ is defined by

$$
\theta\left(k_{\hat{\iota}}\right)=\tan ^{-1}\left[\frac{k_{\hat{\iota}}+\mathbb{C} B\left(k_{\hat{\varkappa}}\right)}{\sqrt{\mathbb{C}} m+\mathbb{C} A\left(k_{\hat{\varkappa}}\right)}\right] .
$$

By comparing Eq. (58) with Eq. (53), we can identify

$$
A\left(p_{\hat{\varkappa}}\right)=\frac{\lambda}{2} f \frac{d k_{\hat{\varkappa}}}{\left(p_{\hat{\iota}}-k_{\hat{\iota}}\right)^{2}} \cos \theta\left(k_{\hat{\iota}}\right)
$$

and

$$
B\left(p_{\varkappa}\right)=\frac{\lambda}{2} f \frac{d k_{\hat{\lrcorner}}}{\left(p_{\hat{\iota}}-k_{\varkappa}\right)^{2}} \sin \theta\left(k_{\varkappa}\right) .
$$

From Eq. (59), we may geometrically represent the effective mass and longitudinal momentum of the particle

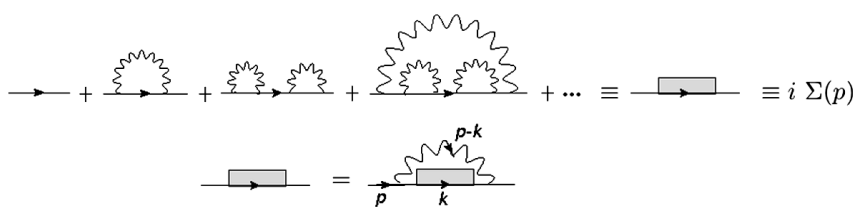

FIG. 1. Self-energy equation.

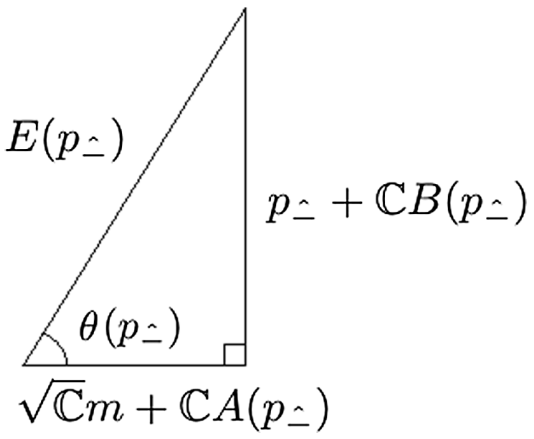

FIG. 2. Geometrical representation of mass gap equations representing Eqs. (62)-(64). moving in nontrivial vacuum by drawing the triangle picture shown in Fig. 2. From Fig. 2, we identify the energy $E\left(p_{\wedge}\right)$ as

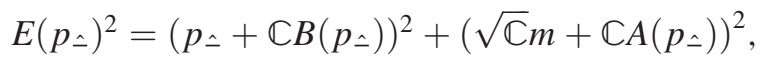

and find the mass gap equations

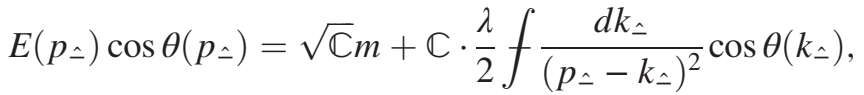

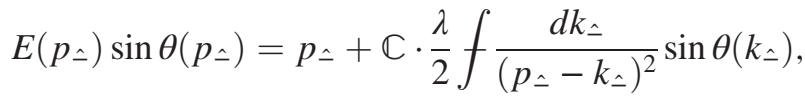

where we used Eqs. (60) and (61) for $A\left(p_{\hat{\iota}}\right)$ and $B\left(p_{\hat{\iota}}\right)$. Now, multiplying Eq. (63) by $\sin \theta\left(p_{\hat{\Lambda}}\right)$ and Eq. (64) by $\cos \theta\left(p_{\iota}\right)$ and subtracting them, we get the exact same equation in Sec. II A as given by Eq. (51). Also, by multiplying Eq. (63) by $\cos \theta\left(p_{\wedge}\right)$ and Eq. (64) by $\sin \theta\left(p_{\wedge}\right)$ and adding them, we get the same equation as Eq. (48) in Sec. II A.

In Sec. III, we numerically solve Eq. (51) to obtain $\theta\left(p_{\hat{\sim}}\right)$ and plug it into Eq. (48) to find $E\left(p_{\wedge}\right)$. We note that the solution of $E\left(p_{\wedge}\right)$ is not always positive and thus the geometric interpretation of Fig. 2 should be regarded as a pictorial device to represent Eqs. (62)-(64) without assuming that the lengths of the triangle sides are positive. We also recognize Eq. (57) as $E_{u}$ and $E_{v}$ mentioned in the previous subsection, Sec. II A, i.e.,

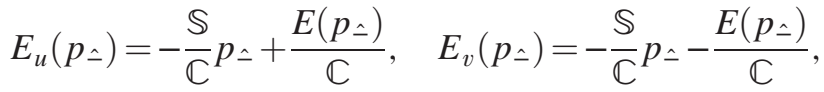

where we note $E_{u}\left(-p_{\hat{\iota}}\right)=-E_{v}\left(p_{\hat{\iota}}\right)$ and $E_{v}\left(-p_{\hat{\iota}}\right)=$ $-E_{u}\left(p_{\hat{\Lambda}}\right)$ due to the evenness of $E\left(p_{\hat{\Lambda}}\right)$ under $p_{\hat{\iota}} \leftrightarrow-p_{\hat{\iota}}$, i.e., $E\left(p_{\hat{\iota}}\right)=E\left(-p_{\hat{\varkappa}}\right)$. When $E\left(p_{\hat{\varkappa}}\right)$ is 
positive, $E_{u}$ is the first energy pole corresponding to the plus sign in Eq. (57), and $E_{v}$ to the minus sign as written in Eq. (65). When $E\left(p_{\lrcorner}\right)$is negative, however, $E_{u}$ is the pole with minus sign, and $E_{v}$ the plus sign. Moreover, one can naturally obtain Eqs. (47) and (49) by adding and subtracting $E_{u}$ and $E_{v}$ in Eq. (65).

\section{Behavior of the gap equation when approaching the light front}

We note that the interpolating mass gap equations are greatly simplified in the limit to the LFD, i.e., $\mathbb{C} \rightarrow 0$. In this limit, Eq. (51) becomes

$$
p^{+} \cos \theta\left(p^{+}\right)=0
$$

where one should note $p_{\text {^ }}=p_{-}=p^{+}$as $\mathbb{C} \rightarrow 0$. The solution of Eq. (66) is analytically given by

$$
\theta\left(p^{+}\right)=\frac{\pi}{2} \operatorname{sgn}\left(p^{+}\right)
$$

Likewise, Eq. (48) is simplified as

$$
E\left(p^{+}\right)=p^{+} \sin \theta\left(p^{+}\right) .
$$

Moreover, Eqs. (60) and (61) are now given by

$$
\begin{gathered}
A\left(p^{+}\right)=0\left(\text { except } p^{+}=0\right), \\
B\left(p^{+}\right)=\frac{\lambda}{2} f \frac{d k^{+}}{\left(p^{+}-k^{+}\right)^{2}} \operatorname{sgn}\left(k^{+}\right),
\end{gathered}
$$

where the light-front zero-mode $p^{+}=0$ contribution should be taken into account separately with the form of $A\left(p^{+}\right)=A(0) \delta\left(p^{+}\right)$solution in mind. Besides the $p^{+}=0$ contribution, it is interesting to note a remarkable simplification of the self-energy in the LFD given by $\Sigma\left(p^{+}\right)=$ $\gamma^{+} B\left(p^{+}\right)$due to the absence of the scalar part, i.e., $A\left(p^{+}\right)=0$. For the computation of $B\left(p^{+}\right)$in Eq. (70), 't Hooft [1] did not use the principal value but discussed how to make the infrared region finite by introducing the infrared cutoff parameter $\varepsilon$ as summarized below for $p^{+}>0$ and $p^{+}<0$, respectively, i.e., in the limit $\varepsilon \rightarrow 0$, for $p^{+}>0$,

$$
\begin{aligned}
B\left(p^{+}\right)= & \frac{\lambda}{2}\left(\int_{-\infty}^{0}-\frac{d k^{+}}{\left(p^{+}-k^{+}\right)^{2}}+\int_{0}^{p^{+}-\varepsilon} \frac{d k^{+}}{\left(p^{+}-k^{+}\right)^{2}}\right. \\
& \left.+\int_{p^{+}+\varepsilon}^{+\infty} \frac{d k^{+}}{\left(p^{+}-k^{+}\right)^{2}}\right) \\
= & -\lambda\left(\frac{1}{p^{+}}-\frac{1}{\varepsilon}\right),
\end{aligned}
$$

while for $p^{+}<0$,

$$
\begin{aligned}
B\left(p^{+}\right)= & \frac{\lambda}{2}\left(\int_{-\infty}^{p^{+}-\varepsilon}-\frac{d k^{+}}{\left(p^{+}-k^{+}\right)^{2}}+\int_{p^{+}+\varepsilon}^{0}-\frac{d k^{+}}{\left(p^{+}-k^{+}\right)^{2}}\right. \\
& \left.+\int_{0}^{+\infty} \frac{d k^{+}}{\left(p^{+}-k^{+}\right)^{2}}\right) \\
= & -\lambda\left(\frac{1}{p^{+}}+\frac{1}{\varepsilon}\right) .
\end{aligned}
$$

Thus, the 't Hooft's solution for $B\left(p^{+}\right)$is given by

$$
B\left(p^{+}\right)=\lambda\left(\frac{\operatorname{sgn}\left(p^{+}\right)}{\varepsilon}-\frac{1}{p^{+}}\right) .
$$

With these solutions, the replacement given by Eq. (55) in the LFD limit $\left(\delta \rightarrow \frac{\pi}{4}\right)$ becomes

$$
\left\{\begin{array} { l } 
{ p _ { \hat { + } } \rightarrow p _ { \hat { + } } - \mathbb { S } B } \\
{ p _ { \hat { \iota } } \rightarrow p _ { \hat { \iota } } + \mathbb { C } B \stackrel { \mathbb { C } \rightarrow 0 } { \rightarrow } } \\
{ m \rightarrow m + \sqrt { \mathbb { C } } A }
\end{array} \left\{\begin{array}{l}
p^{-} \rightarrow p^{-}+\frac{\lambda}{p^{+}}-\lambda \frac{\operatorname{sgn}\left(p^{+}\right)}{\varepsilon} \\
p^{+} \rightarrow p^{+} \\
m \rightarrow m,
\end{array}\right.\right.
$$

and it leads to the dressed fermion propagator given by

$$
\begin{aligned}
S(p) & =\frac{\gamma^{+}\left(p^{-}+\frac{\lambda}{p^{+}}-\lambda \frac{\operatorname{sgn}\left(p^{+}\right)}{\varepsilon}\right)+\gamma^{-} p^{+}+m}{2 p^{+} p^{-}-m^{2}+2 \lambda-2 \lambda \frac{\left|p^{+}\right|}{\varepsilon}+i \epsilon} \\
& =\frac{\gamma^{+}\left(p^{-}-p_{\mathrm{on}}^{-}+\frac{\lambda}{p^{+}}-\lambda \frac{\operatorname{sgn}\left(p^{+}\right)}{\varepsilon}\right)+\gamma^{+} p_{\mathrm{on}}^{-}+\gamma^{-} p^{+}+m}{2 p^{+}\left(p^{-}-p_{\mathrm{on}}^{-}+\frac{\lambda}{p^{+}}-\lambda \frac{\operatorname{sgn}\left(p^{+}\right)}{\varepsilon}\right)+i \epsilon} \\
& =\frac{\gamma^{+}}{2 p^{+}}+\frac{\not \mathrm{on}+m}{p^{2}-m^{2}+2 \lambda-2 \lambda \frac{\left|p^{+}\right|}{\varepsilon}+i \epsilon},
\end{aligned}
$$

where $p_{\mathrm{on}}^{-}=\frac{m^{2}}{2 p^{+}}$and $p_{\mathrm{on}}^{+}=p^{+}$. Here, we note the splitting of the instantaneous contribution $\left(\sim \gamma^{+}\right)$and the so-called on-mass-shell part $\sim\left(\not p_{\text {on }}+m\right)$ of the fermion propagator in LFD. While the interpolating fermion propagator can split into the forward moving part with the energy denominator $1 /\left(p_{\hat{+}}-E_{u}\left(p_{\hat{\iota}}\right)\right)$ and the backward moving part with the energy denominator $1 /\left(p_{\hat{+}}-E_{v}\left(p_{\hat{\iota}}\right)\right)$ as we discuss the details of the fermion propagator in Sec. IV B, one can notice the behaviors of $E_{u}\left(p_{\hat{\iota}}\right)$ and $E_{v}\left(p_{\hat{\iota}}\right)$ in the limit $\mathbb{C} \rightarrow 0$ as $E_{u}\left(p_{\hat{\iota}}\right) \rightarrow B\left(p^{+}\right)+\frac{m^{2}}{2 p^{+}}$and $E_{v}\left(p_{\hat{\iota}}\right) \rightarrow-\left(\frac{2 p^{+}}{\mathbb{C}}+B\left(p^{+}\right)+\right.$ $\left.\frac{m^{2}}{2 p^{+}}\right)$for $p^{+}>0$ while $E_{u}\left(-p_{\varkappa}\right) \rightarrow+\left(\frac{2 p^{+}}{\mathbb{C}}+B\left(p^{+}\right)+\frac{m^{2}}{2 p^{+}}\right)$ and $E_{v}\left(-p_{\hat{\iota}}\right) \rightarrow-\left(B\left(p^{+}\right)+\frac{m^{2}}{2 p^{+}}\right)$for $p^{+}<0$ using Eqs. (62) and (65). Taking $p^{+}>0$ for the sake of discussion, one can rather easily identify that the on-mass-shell part $\sim\left(\not p_{\text {on }}+m\right)$ of the dressed fermion propagator corresponds to the forward moving part from the 't Hooft solution for $B\left(p^{+}\right)$given by Eq. (73) as well as the correspondence $\not_{\mathrm{on}}+$ $m$ with the spinor biproduct $u\left(p^{+}\right) \bar{u}\left(p^{+}\right)$of the dressed fermion. Likewise, one can identify the instantaneous contribution $\left(\sim \gamma^{+}\right)$to the backward moving part by noting the 
cancellation of $1 / \mathbb{C}$ factor in $E_{v}\left(p_{\lrcorner}\right) \sim-\frac{2 p^{+}}{\mathbb{C}}$ with the $1 / \mathbb{C}$ factor in the backward moving spinor biproduct $v\left(-p^{+}\right) \bar{v}\left(-p^{+}\right) \sim \frac{2 p^{+} \gamma^{+}}{\mathbb{C}}$. However, the instantaneous contribution effectively vanishes in the actual calculation of the rainbow and ladder diagrams as every quark line is multiplied by the vertex factor $g \gamma^{+}$from both sides and $\left(\gamma^{+}\right)^{2}=0$. Moreover, as $\left\{\gamma^{+}, \gamma^{-}\right\}=2$, the dressed quark propagator can be effectively given by

$$
S(p)=\frac{p^{+}}{2 p^{+} p^{-}-m^{2}+2 \lambda-2 \lambda \frac{\left|p^{+}\right|}{\varepsilon}+i \epsilon},
$$

with the effective vertex factor $2 g$. We can now see that, due to the $1 / \varepsilon$ infrared divergence, the on-mass-shell pole of this dressed quark propagator moves toward the infinity from the on-mass-shell pole $p_{\text {on }}^{-}=\frac{m^{2}}{2 p^{+}}$. This disappearance of the onmass-shell pole due to the infrared cutoff term was interpreted as the confinement of the fermions in the 't Hooft model [1].

As shown in Ref. [1], however, the infrared cutoff terms cancel themselves in the bound-state spectroscopy calculation. Thus, one can use the principal value prescription as defined in Eq. (43) to regulate the infinite piece. With the same principal value prescription, $B\left(p^{+}\right)$in the limit of $\varepsilon \rightarrow 0$ is given by

$$
\begin{aligned}
B\left(p^{+}\right)= & \frac{\lambda}{2} \int \frac{d k^{+}}{\left(p^{+}-k^{+}\right)^{2}}\left(\operatorname{sgn}\left(k^{+}\right)-\operatorname{sgn}\left(p^{+}\right)\right) \\
= & \lambda\left(\frac{\operatorname{sgn}\left(p^{+}\right)}{\varepsilon}-\frac{1}{p^{+}}\right)-\frac{\lambda}{2} \operatorname{sgn}\left(p^{+}\right) \\
& \times\left(\int_{-\infty}^{p^{+}-\varepsilon} \frac{d k^{+}}{\left(p^{+}-k^{+}\right)^{2}}+\int_{p^{+}+\varepsilon}^{+\infty} \frac{d k^{+}}{\left(p^{+}-k^{+}\right)^{2}}\right) \\
= & -\frac{\lambda}{p^{+}} .
\end{aligned}
$$

The reduced quark propagator without the $1 / \varepsilon$ infrared divergence factor in the denominator is then given by

$$
S(p)=\frac{p^{+}}{2 p^{+} p^{-}-m^{2}+2 \lambda+i \epsilon},
$$

which leads to the same effective planar Feynman rule of the fermion propagator in the light-front gauge presented in Ref. [1] besides the notation difference explained in the footnote in Sec. I.

\section{THE MASS GAP SOLUTION}

The mass gap equation given by Eq. (51) is solved numerically using a generalized Newton method mentioned in Ref. [11] as well as in Ref. [12], and we use the same numerical method elaborated in Refs. [11,12]. Using the same 200 grid points and choosing the same quark mass values (in unit of $\sqrt{2 \lambda}$ ) as in Ref. [11] and in Ref. [12], we obtain the numerical solutions of Eq. (51). To cover the entire interpolating longitudinal momentum range, $-\infty<p_{\hat{\imath}}<+\infty$, we use the variable $\xi=\tan ^{-1} p_{\hat{\iota}}$, where $\xi \in\left(-\frac{\pi}{2}, \frac{\pi}{2}\right)$. As the solutions of $\theta\left(p_{-}\right)$are antisymmetric under the transformation of $p_{\hat{\Lambda}} \rightarrow-p_{\hat{\Lambda}}$, i.e., $\theta\left(-p_{\hat{\Lambda}}\right)=$ $-\theta\left(p_{\hat{A}}\right)$, we present the results for the region $0<p_{\text {А }}<$ $+\infty$ only, i.e., $0<\xi<\frac{\pi}{2}$, for several quark mass values in

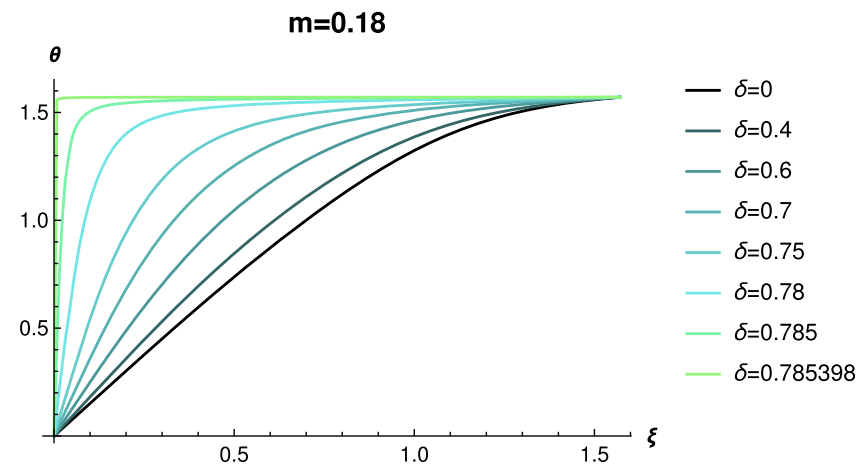

(a)
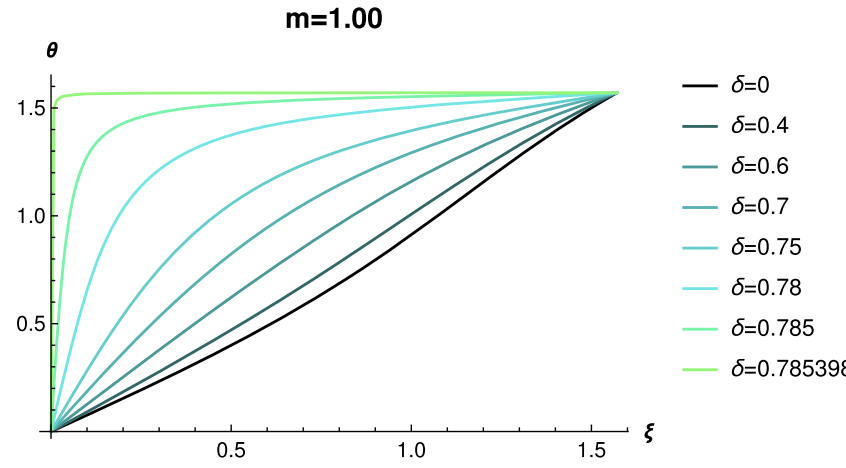

(b)

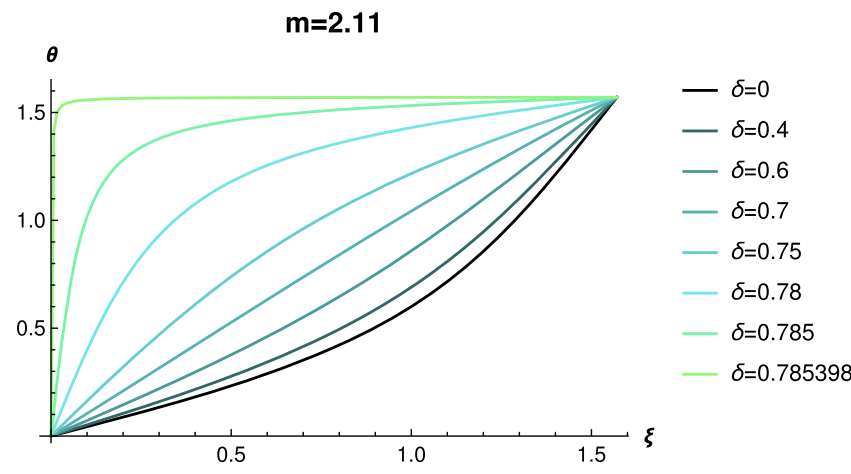

(c)

FIG. 3. The numerical solutions of $\theta\left(p_{\wedge}\right)$ for several interpolation angles, corresponding to the quark mass values in Fig. 4 of Ref. [11]. All the mass values are in the unit of $\sqrt{2 \lambda}$. Note that $\theta\left(p_{\hat{\iota}}\right)$ is an odd function of $p_{\hat{\imath}}$ and only the positive $p_{\hat{\imath}}$ range is plotted with the variable $\xi=\tan ^{-1} p_{\hat{\wedge}}$. The closeness of $\delta=0.785398$ to $\pi / 4$ can be assessed by the ratio $\frac{0.785398}{\pi / 4} \approx 0.999999792$. 


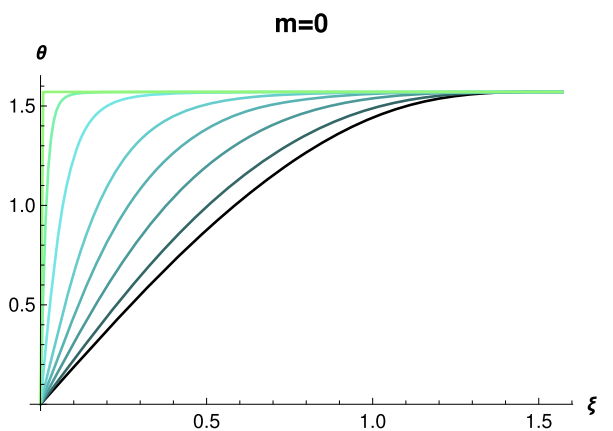

(a)

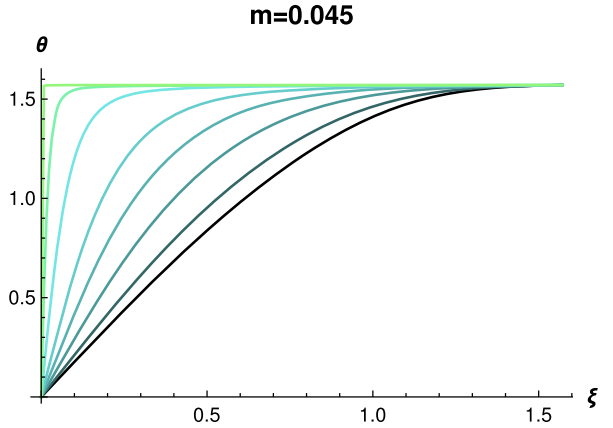

(b)

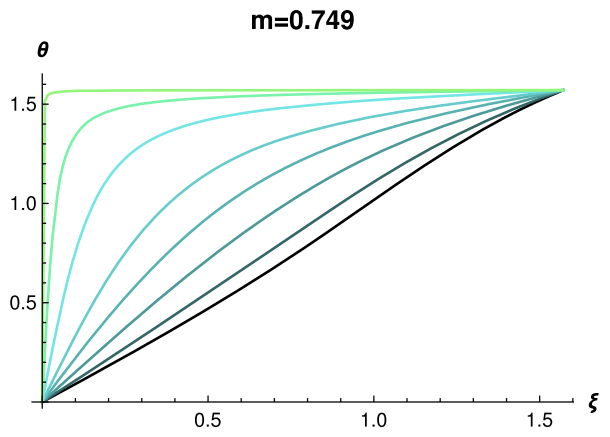

(c)

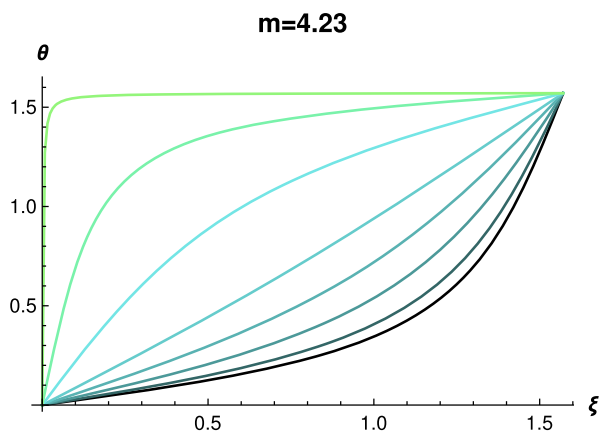

(d)

FIG. 4. The numerical solutions of $\theta\left(p_{\hat{\iota}}\right)$ for several interpolation angles, corresponding to the quark mass values in Fig. 2 of Ref. [12]. All the mass values are in the unit of $\sqrt{2 \lambda}$. Note that $\theta\left(p_{\hat{\iota}}\right)$ is an odd function of $p_{\hat{\imath}}$ and only the positive $p_{\hat{\imath}}$ range is plotted with the variable $\xi=\tan ^{-1} p_{\hat{\iota}}$. The closeness of $\delta=0.785398$ to $\pi / 4$ can be assessed by the ratio $\frac{0.785398}{\pi / 4} \approx 0.999999792$.

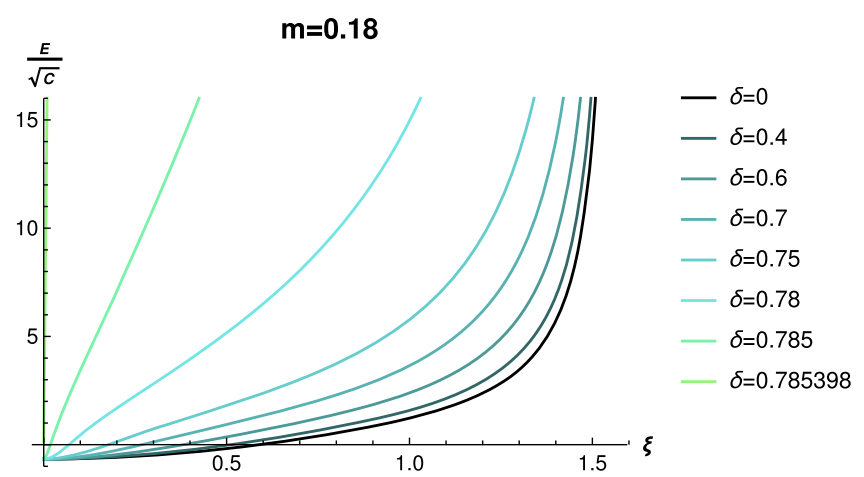

(a)

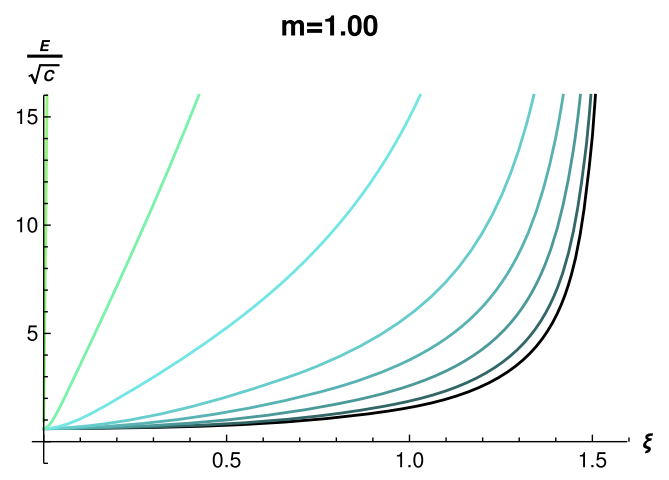

(b)

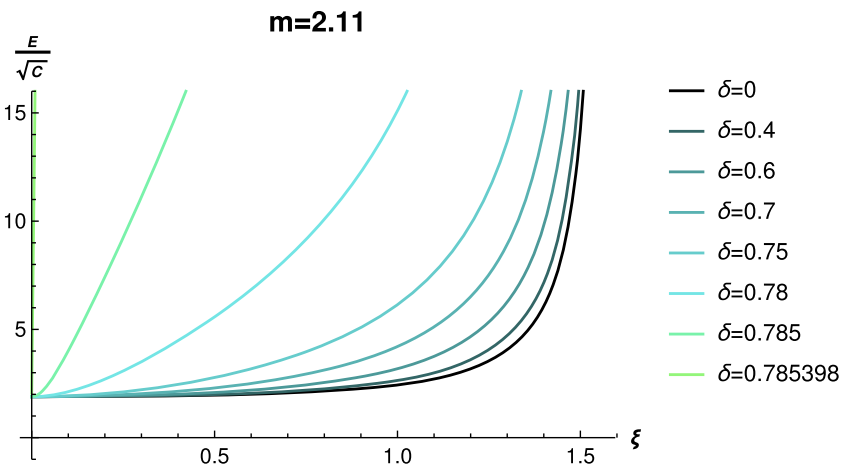

(c)

FIG. 5. The solutions of $E\left(p_{\hat{\wedge}}\right) / \sqrt{\mathbb{C}}$ for several interpolation angles for different choices of quark mass corresponding to the quark mass values in Fig. 5 of Ref. [11]. All quantities are in proper units of $\sqrt{2 \lambda} . E\left(p_{\hat{\iota}}\right)$ is an even function of $p_{\hat{\iota}}$. We plot only for positive $p_{\hat{\iota}}$ with the variable $\xi=\tan ^{-1} p_{\hat{\iota}}$.

Figs. 3 and 4. In Fig. 3, the numerical solutions of $\theta\left(p_{\wedge}\right)$ for several interpolation angles are plotted for the quark mass values presented in Ref. [11]. The profiles of $\theta(\xi)$ for $\delta=0$ coincide with the IFD results provided in Ref. [11]'s Fig. 4. Also, the profiles of $\theta(\xi)$ for $\delta \rightarrow \pi / 4$ approach to the analytic solution given by Eq. (67) in LFD. It is interesting to note that the IFD results exhibit a convex profile for the lighter quark mass $(m=0.18)$, and as the quark mass increases $(m=1.00$ and 2.11$)$ the profile gets more and 
more concave. For the quark mass values presented in Ref. [12], the numerical solutions of $\theta\left(p_{\wedge}\right)$ for several interpolation angles are plotted in Fig. 4. The authors of Ref. [12] chose the ' $t$ Hooft coupling $\lambda$ as $\pi \lambda=0.18 \mathrm{GeV}^{2}$ in conformity to the value of the string tension in the realistic $\mathrm{QCD}_{4}$, and then determined the light quark mass $m_{u / d}=$ 0.045 to obtain the physical pion mass $M_{\pi}=0.41$ in the unit of $\sqrt{2 \lambda}$ by solving the bound-state equation, which we present in Sec. V. Likewise, the heavy quark mass $m_{c}=4.23$ was determined to get the physical $J / \psi$ mass $M_{J / \psi}=9.03$ in the same unit. The strange quark mass $m_{s}=0.749$ was taken to provide a threshold, below (above) which is called light (heavy) flavor, by minimizing the relative distance between the $\theta(\xi)$ solution and the straight line $\theta(\xi)=\xi$ in IFD as the profile of $\theta(\xi)$ in IFD (i.e., $\delta=0$ ) passes from the convex to concave with the increasing quark mass as discussed in Fig. 3. The chiral limit with $m_{u}=0$ was also considered and the Gell-Mann-Oakes-Renner relation (GOR) was discussed in Ref. [12]. For those quark masses, $m=0$, $0.045,0.749$ and 4.23 , we obtain the interpolating mass gap solutions for several $\delta$ values between $\delta=0$ (IFD) and $\delta=\pi / 4$ (LFD) as plotted in Fig. 4. Our numerical solutions for $\delta=0$ coincide with the IFD results provided in Ref. [12]'s Fig. 2 (left panel). Again, the profiles of $\theta(\xi)$ for $\delta \rightarrow \pi / 4$ approach to the analytic solution given by Eq. (67) in LFD.

We then obtain the solutions for $E\left(p_{\hat{A}}\right)$ by plugging the mass gap solutions presented in Figs. 3 and 4 into Eq. (48). As the plots of $E\left(p_{\hat{\wedge}}\right)$ themselves for different interpolation angles are too close to each other to observe clearly, we present the solutions of $E\left(p_{\lrcorner}\right) / \sqrt{\mathbb{C}}$ in Figs. 5 and 6. The quark mass values in Figs. 5 and 6 correspond to those in Figs. 3 and 4 , respectively. At $\delta=0$, the profiles of $E\left(p_{\hat{\varkappa}}\right) / \sqrt{\mathbb{C}}=E\left(p^{1}\right)$ in Figs. 5 and 6 coincide with the IFD results provided in Ref. [11]'s Fig. 5 and Ref. [12]'s Fig. 2 (right panel), respectively. Examining the results of $E\left(p_{\wedge}\right)$ themselves in the limit $\delta \rightarrow \pi / 4$, we also find that all of them approach to the analytic result of $E\left(p^{+}\right)$given by Eq. (68) independent of quark mass $m$. Moreover, the LFD result of $E\left(p^{+}\right)$given by Eq. (68) is always positive regardless of $p^{+}$while the IFD $(\delta=0)$ results of $E\left(p^{1}\right)$ for small quark mass values, e.g., $m=0.18$ in Fig. 5(a) and $m=0$ and 0.045 in Figs. 6(a) and 6(b), respectively, are negative for small momentum regions. Indeed, we notice that the region of small momentum for the negative value of $E\left(p^{1}\right)$ gets shrunken as $m$ gets larger but persists up to $m \approx 0.56$. It was argued in Ref. [10] that the existence of negative quark self-energy does not cause any concern though, due to the lack of observability for the energy of a confined single quark. While similar aspect of the negative quark energy exists for other interpolation angle values unless $\delta=\pi / 4$, the corresponding range of the small momentum gets reduced as $\delta$ approaches to $\pi / 4$ as depicted in Figs. 5(a), 6(a), and 6(b). It is interesting to note that our

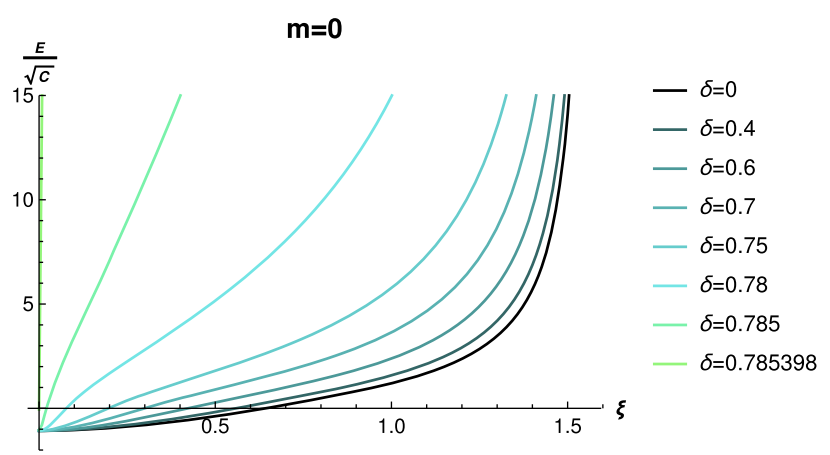

(a)

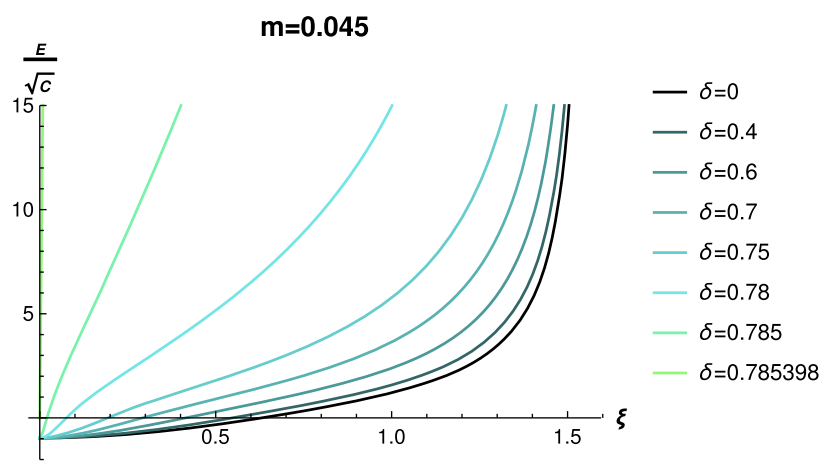

(b)

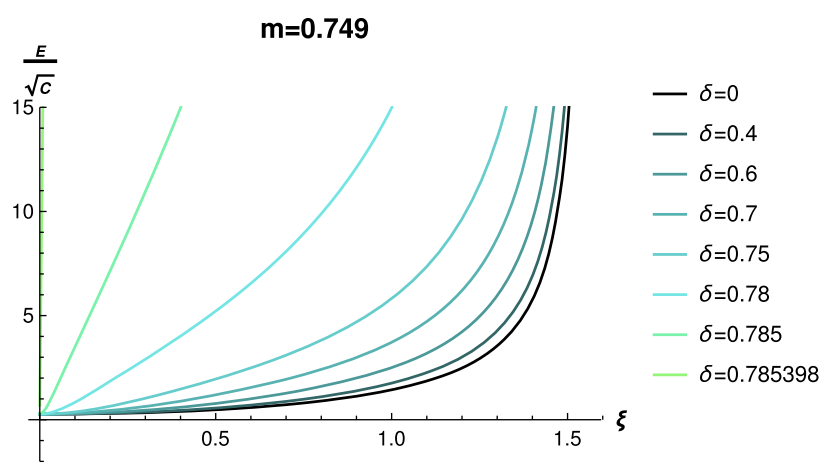

(c)

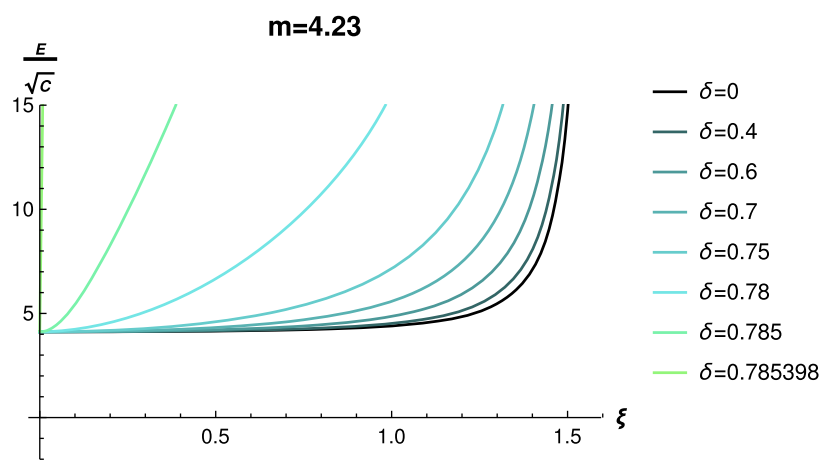

(d)

FIG. 6. The solutions of $E\left(p_{\hat{\wedge}}\right) / \sqrt{\mathbb{C}}$ for several interpolation angles for different choices of quark mass, corresponding to the quark mass values in Fig. 2 of Ref. [12]. All quantities are in proper units of $\sqrt{2 \lambda} . E\left(p_{\hat{\iota}}\right)$ is an even function of $p_{\hat{\iota}}$. We plot only for positive $p_{\hat{\wedge}}$ with the variable $\xi=\tan ^{-1} p_{\hat{\iota}}$. 
observation of diminishing the negative quark self-energy region in the limit $\delta \rightarrow \pi / 4$ (LFD) appears consistent with the result that the single particle energies do not change sign due to the additional constant term appearing in converting the light-front longitudinal momentum sum to a principal value integral discussed in Ref. [25] with the formulation of a finite light-front $x^{-}$coordinate interval.

\section{CHIRAL CONDENSATE AND CONSTITUENT QUARK MASS}

As we have obtained the mass gap solutions, we now utilize them to discuss the chiral condensate and the constituent quark mass in this section.

\section{A. The chiral condensate}

While the Coleman's theorem [26] prohibits the spontaneously broken chiral symmetry (SBCS) in two-dimensional theories with a finite number of degrees of freedom, the large $N_{c}$ limit of the 't Hooft model does not contradict with the Coleman's theorem. The exact result for the chiral condensate was found in the chiral limit $(m \rightarrow 0)$ for the weak coupling regime of $\mathrm{QCD}_{2}\left(m \gg g \sim 1 / \sqrt{N_{c}}\right)$ [4] as

$$
\langle\bar{\psi} \psi\rangle=-N_{c} / \sqrt{12}
$$

in the unit of the mass dimension $\sqrt{2 \lambda}$ with the definition of $\lambda$ given by Eq. (35). This indicates that the SBCS occurs in the 't Hooft model, in contrast to the strong coupling regime of $\mathrm{QCD}_{2}$ in which the SBCS does not occur according to the Coleman's theorem [26]. Ramifications of the nontrivial chiral condensates with respect to the vacuum in the LFD as well as its nonanalytic behavior were discussed for nonzero quark masses and its chiral limit [27]. For nonzero quark masses, the renormalized quark condensation was defined by subtracting the free field expectation value to render the quark condensation finite [27],

$$
\left.\langle\bar{\psi} \psi\rangle\right|_{\text {ren }} \equiv\langle\bar{\psi} \psi\rangle-\left.\langle\bar{\psi} \psi\rangle\right|_{g=0} .
$$

In the chiral limit, $m \rightarrow 0,\left.\langle\bar{\psi} \psi\rangle\right|_{g=0}=0$ and thus $\left.\langle\bar{\psi} \psi\rangle\right|_{\text {ren }}=\langle\bar{\psi} \psi\rangle$. The numerical computation verifying Eq. (79) was presented in IFD [28] substituting the mass gap solution for $\theta\left(p^{1}\right)$ in the chiral limit $m \rightarrow 0$ as

$$
\left.\langle\bar{\psi} \psi\rangle\right|_{\text {ren }}=-N_{c} \int_{-\infty}^{+\infty} \frac{d p^{1}}{2 \pi} \cos \theta\left(p^{1}\right) \approx-0.29 N_{c} .
$$

With the interpolating quark field between IFD and LFD given by Eq. (20), we find

$$
\begin{aligned}
& \left.\langle\bar{\psi} \psi\rangle\right|_{\text {ren }}=N_{c} \int_{-\infty}^{+\infty} \frac{d p_{\text {合 }}}{(2 \pi)\left(2 p^{\hat{+}}\right)} \\
& \times\left[\operatorname{Tr}\left\{v\left(-p_{\hat{\iota}}\right) \bar{v}\left(-p_{\hat{\iota}}\right)\right\}-\operatorname{Tr}\left\{v^{(0)}\left(-p_{\hat{\iota}}\right) \bar{v}^{(0)}\left(-p_{\hat{\iota}}\right)\right\}\right] \\
& =-\frac{N_{c}}{2 \pi \sqrt{\mathbb{C}}} \int_{-\infty}^{+\infty} d p_{\lrcorner}\left[\cos \theta\left(p_{\wedge}\right)-\cos \theta_{f}\left(p_{\text {^ }}\right)\right],
\end{aligned}
$$

where we have used the interpolating spinors given by Eqs. (29) and (31). We numerically compute Eq. (82) in the chiral limit, $m \rightarrow 0$, for different interpolation angles, and the results are shown in Table I. We observe that the closer one gets to the LFD $\left(\delta=\frac{\pi}{4}\right)$, the higher accuracy one needs for the numerical computation and thus we list the results obtained by increasing the number of grid points used. One can see that when $\delta$ is away from $\pi / 4$, the coarser grid is already good enough to obtain an accurate result. For $\delta$ closer to $\pi / 4$, the number of grid points should be increased to improve the numerical accuracy. Table I indicates an eventual agreement to the analytical value $-1 / \sqrt{12} \approx$ -0.29 regardless of the interpolation angle $\delta$ with the enhancement of the numerical accuracy.

Even if the chiral limit is not taken, we notice that the renormalized chiral condensate must be independent of the interpolating angle $\delta$ as it must be the characteristic quantity determining the vacuum property for a given phase of the theory. In fact, the interpolating longitudinal momentum variable $p_{\text {s }}$ can be scaled out by the interpolating parameter $\sqrt{\mathbb{C}}$ and Eq. (82) can be given by the rescaled variable $p_{\hat{\iota}}^{\prime}=p_{\iota} / \sqrt{\mathbb{C}}$, i.e.,

TABLE I. The numerically calculated condensation values in the chiral limit with different interpolation angles and computational accuracy. All quantities are in proper units of $\sqrt{2 \lambda}$.

\begin{tabular}{lcc}
\hline \hline$\delta$ & Number of grid points & $\left.\langle\bar{\psi} \psi\rangle\right|_{m \rightarrow 0} / N_{c}$ \\
\hline 0 & 200 & -0.285209 \\
& 600 & -0.287508 \\
0.4 & 200 & -0.285164 \\
& 600 & -0.287496 \\
0.6 & 200 & -0.284792 \\
& 600 & -0.287375 \\
0.7 & 200 & -0.283836 \\
& 600 & -0.287059 \\
0.75 & 200 & -0.281837 \\
& 600 & -0.286396 \\
0.78 & 200 & -0.296575 \\
& 600 & -0.291334 \\
0.785 & 600 & -0.298104 \\
& 1000 & -0.294377 \\
0.78535 & 3000 & -0.290590 \\
& 1000 & -0.304659 \\
& 3000 & -0.294134 \\
\hline \hline
\end{tabular}




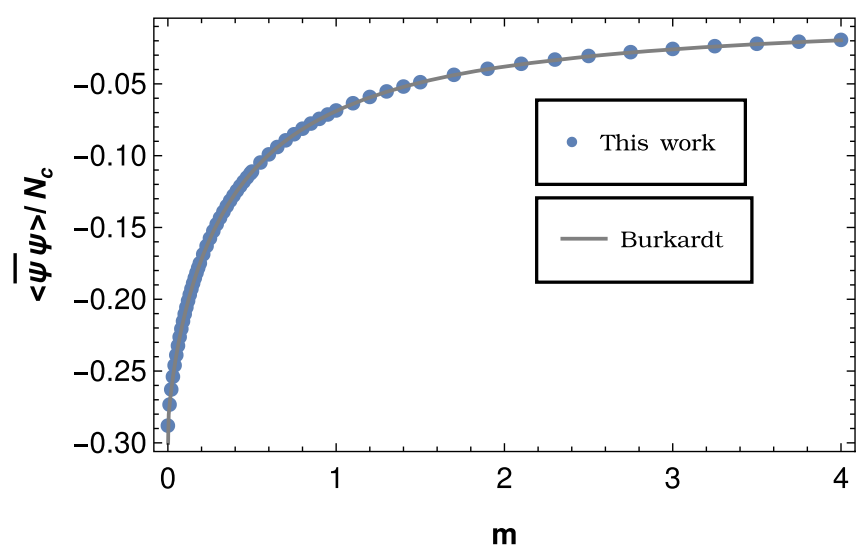

FIG. 7. Numerical results of the condensation $\left.\langle\bar{\psi} \psi\rangle\right|_{\text {ren }}$ as a function of $m$ in comparison with the analytic result in Ref. [27]. All quantities are in proper units of $\sqrt{2 \lambda}$.

$\left.\langle\bar{\psi} \psi\rangle\right|_{\text {ren }}=-\frac{N_{c}}{2 \pi} \int_{-\infty}^{+\infty} d p_{\wedge}^{\prime}\left[\cos \theta\left(p_{\wedge}^{\prime}\right)-\cos \theta_{f}\left(p_{\wedge}^{\prime}\right)\right]$.

The same variable change can be applied to the interpolating mass gap equation given by Eq. (51) as we illustrate in Appendix $\mathrm{C}$ to obtain the rescaled mass gap equation without any apparent interpolation angle dependence as given by Eq. (C6). With $\theta\left(p_{\dot{A}}^{\prime}\right)$ being the solution of Eq. (C6) as well as $\theta_{f}\left(p_{\lrcorner}^{\prime}\right)=\tan ^{-1}\left(p_{\lrcorner}^{\prime} / m\right)$ from Eq. (27), we can confirm that the chiral condensate is indeed independent of the interpolation angle $\delta$. Thus, the result of the chiral condensate must be identical whichever dynamics is chosen for the computation between $\delta=0$ (IFD) and $\delta=\pi / 4$ (LFD). Computing $\left.\langle\bar{\psi} \psi\rangle\right|_{\text {ren }}$ in Eq. (82) for any given interpolation angle between $\delta=0$ and $\delta=\pi / 4$, one can numerically verify the uniqueness of the result for each and every quark mass values that one takes. Varying the quark masses between $m=0$ and $m=4$ in the unit of $\sqrt{2 \lambda}$, we computed $\left.\langle\bar{\psi} \psi\rangle\right|_{\text {ren }}$ from Eq. (83) and obtained the numerical result shown in Fig. 7. Our numerical result for $m=0$ in Fig. 7 confirms the results obtained in Table I reproducing the analytic value $-1 / \sqrt{12} \approx-0.29$. Likewise, our numerical results for $0<m \leq 4$ coincide with the analytic result given by Eq. (2.19) of Ref. [27] which was also numerically confirmed in Ref. [12].

In the formulation of a finite light-front $x^{-}$coordinate interval [25], a phase transition was reported in the weak coupling limit as a dramatic change of the quark condensate value to zero was observed. In the continuum limit, however, there is no such phase transition and the nonzero condensate value is intact regardless of the form of dynamics between $\delta=0$ (IFD) and $\delta=\pi / 4$ (LFD) as discussed in this subsection. In this respect, it is important to realize that the phase characterized by the SBCS is uniquely viable in the continuum 't Hooft model. While there is a simple analytic step function solution of Eq. (51) in IFD $(\delta=0)$ given by $\theta\left(p^{1}\right)=\frac{\pi}{2} \operatorname{sgn}\left(p^{1}\right)$ in the chiral limit [10], it should be clearly distinguished from the step function solution in LFD given by Eq. (67). The step function solution in IFD leads to the zero chiral condensate $\langle\bar{\psi} \psi\rangle=0$ in contrast to the nontrivial chiral condensate given by Eq. (79). It was indeed demonstrated in Ref. [29] that this chirally symmetric step function solution in IFD reveals the possession of infinite vacuum energy compared to the vacuum energy of the SBCS solution. Thus, the step function solution in IFD should be clearly distinguished from the physically viable solution characterized by the SBCS [30] discussed in this subsection.

\section{B. The fermion propagator and constituent mass}

The interpolating free fermion propagator has been discussed at length in our previous work [15] and can be readily applied for the interpolating bare quark propagator in $1+1$ dimension with the notation of the on-massshell two-momenta $P_{a \hat{\mu}}=\left(P_{a \hat{+}}, p_{\hat{\iota}}\right), P_{b \hat{\mu}}=\left(-P_{b \hat{+}},-p_{\hat{\varkappa}}\right)$ taking $P_{a \hat{+}}$ and $-P_{b \hat{+}}$ as the positive and negative on-shell interpolating energies of the bare quark, i.e.,

$$
P_{a \hat{+}}=-\frac{\mathbb{S}}{\mathbb{C}} p_{\hat{\imath}}+\frac{\sqrt{p_{\hat{\imath}}^{2}+\mathbb{C}^{2}}}{\mathbb{C}}=\frac{-\mathbb{S} p_{\hat{\iota}}+p^{\hat{+}}}{\mathbb{C}},
$$

and

$$
-P_{b \hat{+}}=-\frac{\mathbb{S}}{\mathbb{C}} p_{\hat{\imath}}-\frac{\sqrt{p_{\hat{\imath}}^{2}+\mathbb{C} m^{2}}}{\mathbb{C}}=\frac{-\mathbb{S} p_{\hat{\iota}}-p^{\hat{+}}}{\mathbb{C}} .
$$

The interpolating bare quark propagator is then given by

$S(p)_{\mathrm{f}}=\frac{1}{2 p^{\hat{+}}}\left(\frac{P_{a}+m}{p_{\hat{+}}-P_{a \hat{+}}+i \epsilon}+\frac{P_{b}-m}{p_{\hat{+}}+P_{b \hat{+}}-i \epsilon}\right)$,

where $\left(\not P_{a}+m\right)=u^{(0)}\left(p_{\iota}\right) \bar{u}^{(0)}\left(p_{\iota}\right)$ and $\quad\left(\not P_{b}-m\right)=$ $v^{(0)}\left(-p_{\lrcorner}\right) \bar{v}^{(0)}\left(-p_{\lrcorner}\right)$can be easily verified using the interpolating free spinors given by Eqs. (30) and (31). In Eq. (86), we call $m$ as the bare quark mass. As discussed in Sec. II B, the interpolating dressed quark propagator can be obtained from the interpolating bare quark propagator with the replacement given by Eq. (55). Effectively, $P_{a \hat{+}}$ and $P_{b \hat{+}}$ are replaced by $E_{u}$ and $E_{v}$ in Eq. (65) as well as the free spinors, $u^{(0)}\left(p_{\hat{\iota}}\right)$ and $v^{(0)}\left(p_{\hat{\varkappa}}\right)$, are replaced by the spinors given by Eqs. (28) and (29), $u\left(p_{\hat{\iota}}\right)$ and $v\left(p_{\hat{\iota}}\right)$, respectively. The dressed quark propagator is then obtained as

$S(p)=\frac{1}{2 p^{\hat{+}}}\left[\frac{u\left(p_{\hat{\iota}}\right) \bar{u}\left(p_{\hat{\iota}}\right)}{p_{\hat{+}}-E_{u}\left(p_{\hat{\iota}}\right)+i \epsilon}+\frac{v\left(-p_{\hat{\iota}}\right) \bar{v}\left(-p_{\hat{\iota}}\right)}{p_{\hat{+}}-E_{v}\left(p_{\hat{\iota}}\right)-i \epsilon}\right]$,

where the first and second terms correspond to the forward and backward moving propagators, respectively. The equivalence of Eq. (87) to Eq. (54) can be verified using the relations 


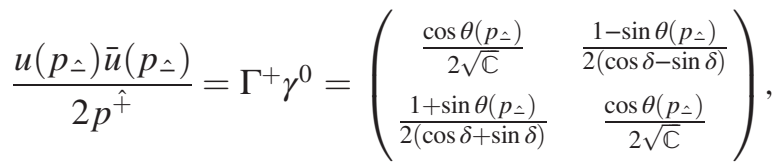

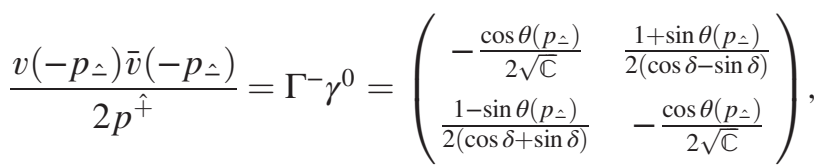

where $\Gamma^{+}$and $\Gamma^{-}$are given by Eqs. (45) and (46), respectively. As discussed in Sec. II C, one can verify that the forward and backward moving parts in the limit $\mathbb{C} \rightarrow 0$ correspond to the on-mass-shell part $\sim\left(\not p_{\text {on }}+m\right)$ and the instantaneous contribution $\left(\sim \gamma^{+}\right)$in LFD, respectively.

To discuss the dressed fermion propagator in more physical terms [31], one can express the dressed quark propagator given by Eq. (54) in terms of the mass function $M\left(p_{\hat{\prime}}\right)$ and the wave function renormalization factor $F\left(p_{\text {^ }}\right)$, i.e.,

$$
S(p)=\frac{F\left(p_{\curlywedge}\right)}{\not p-M\left(p_{\curlywedge}\right)},
$$

and identify $M\left(p_{\hat{\varkappa}}\right)$ and $F\left(p_{\hat{\varkappa}}\right)$ respectively as

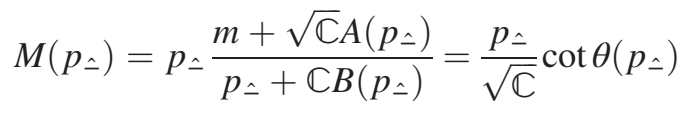

and

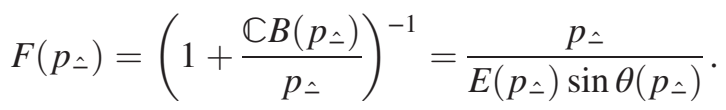

We then numerically compute $M\left(p_{\hat{\iota}}\right)$ and $F\left(p_{\hat{\iota}}\right)$ using the mass gap solutions $\theta\left(p_{\hat{\Lambda}}\right)$ and $E\left(p_{\hat{\Lambda}}\right)$ obtained in Sec. III.

In Figs. 8 and 9, the results of the mass function $M\left(p_{\hat{A}}\right)$ are shown as a function of the variable $\xi=\tan ^{-1} p_{\text {^ }}$ for the bare quark mass values in Ref. [11] $(m=0.18,1.00,2.11)$ and Ref. [12] ( $m=0,0.045,0.749,4.23)$, respectively. As we can see in Figs. 8 and 9, the mass function $M\left(p_{\text {A }}\right)$ approaches the respective bare quark mass value $m$ as $\xi \rightarrow \pi / 2$ or $p_{\hat{\imath}} \rightarrow \infty$ while it gets to the respective characteristic mass value $M(0)$ at $\xi=0$ or $p_{\hat{\imath}}=0$ regardless of the interpolation angle $\delta$, although the profile of $M\left(p_{\wedge}\right)$ in $0<\xi<\pi / 2$ does depend on the value of $\delta$. The characteristic mass value $M(0)$ may be regarded as the dressed quark mass acquired from the dynamical selfenergy interaction depicted in Fig. 1 . We note that the profile of $M\left(p_{\hat{\lrcorner}}\right)$ as $\delta \rightarrow \pi / 4$ approaches to the shape of the step-function which drops from $M(0)$ to $m$ away from $p^{+}=0$.

In Figs. 10 and 11, the results of the wave function renormalization factor $F\left(p_{\hat{\iota}}\right)$ are plotted with the same variable $\xi$ for the bare quark mass values in Ref. [11] $(m=0.18,1.00,2.11)$ and Ref. [12] $(m=0,0.045,0.749$,

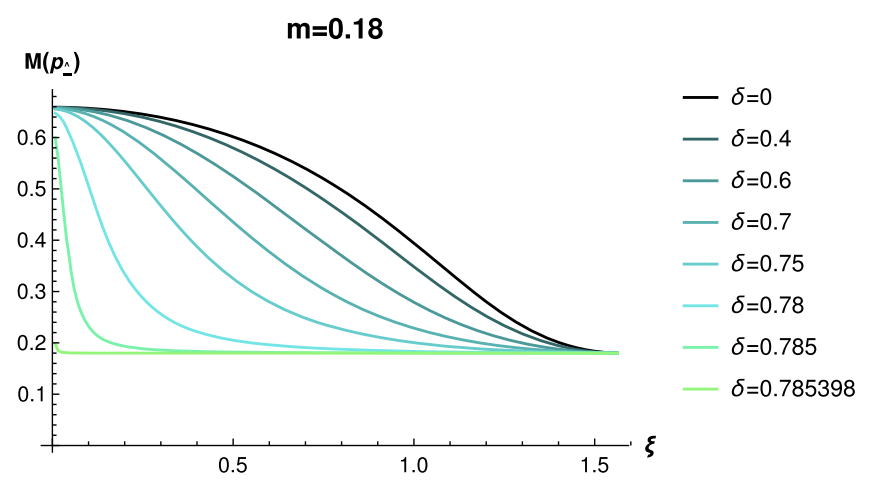

(a)
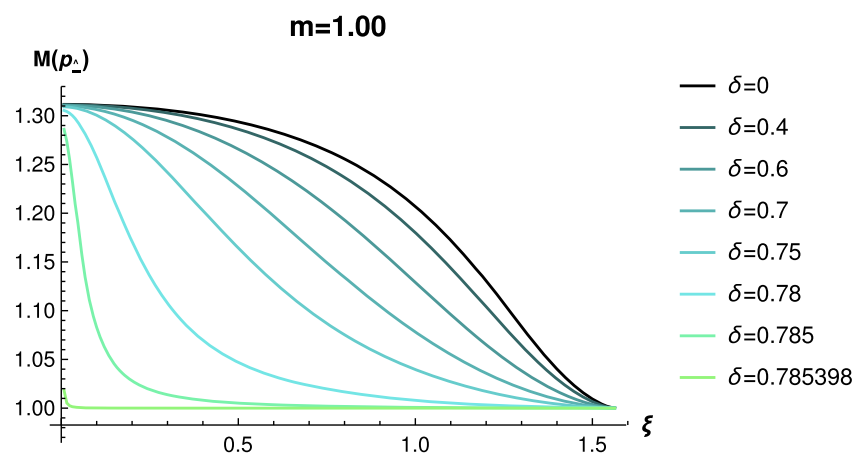

(b)

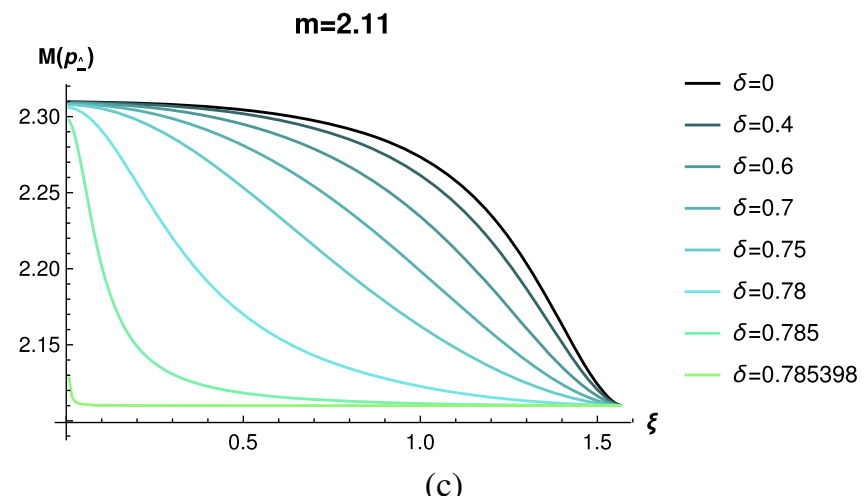

FIG. 8. Constituent mass as a function of $\xi=\tan ^{-1} p_{\text {^ }}$ for (a) $m=0.18$, (b) $m=1.00$, and (c) $m=2.11$. All quantities are in proper units of $\sqrt{2 \lambda}$.

4.23), respectively. Rather immediately, we notice a dramatic difference in the results of $F\left(p_{\hat{\Lambda}}\right)$ for the lower values of bare quark mass $m=0.18$ in Fig. 10 as well as $m=0$ and 0.045 in Fig. 11 due to the negative values for small $\xi=\tan ^{-1} p_{\text {^ }}$ region. Interestingly, the appearance of the negative values in $F\left(p_{\hat{\varkappa}}\right)$ for those bare quark mass values is correlated with the negative values of $E\left(p_{\hat{\varkappa}}\right) / \sqrt{\mathbb{C}}$ discussed previously for Figs. 5(a) $(m=0.18), 6$ (a) $(m=0)$, and 6(b) $(m=0.045)$. To comprehend the sign correlation between $F\left(p_{\hat{\iota}}\right)$ and $E\left(p_{\hat{\iota}}\right)$, we write $\cos \theta\left(p_{\hat{\iota}}\right)$ and $\sin \theta\left(p_{\hat{\iota}}\right)$ in terms of $M\left(p_{\hat{\iota}}\right)$ and $F\left(p_{\hat{\iota}}\right)$ from Eqs. (89) and (90), as 


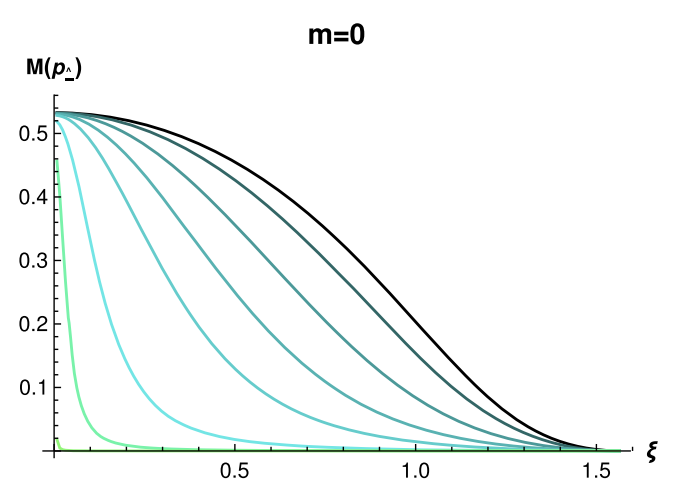

(a)

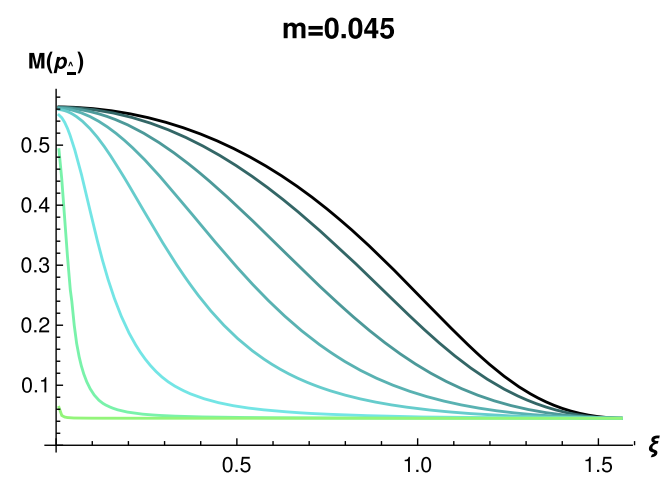

(b)

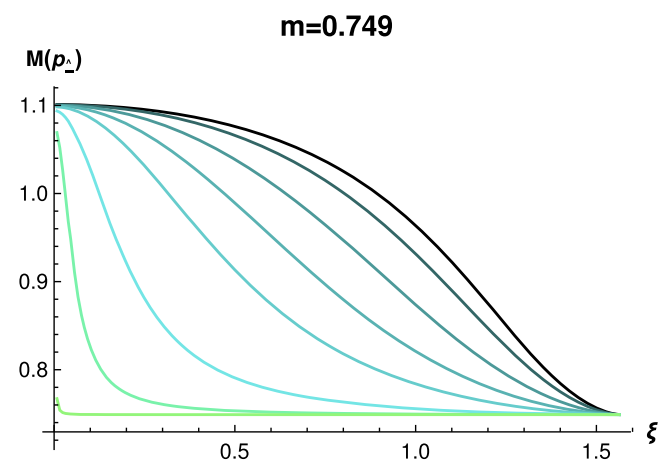

(c)
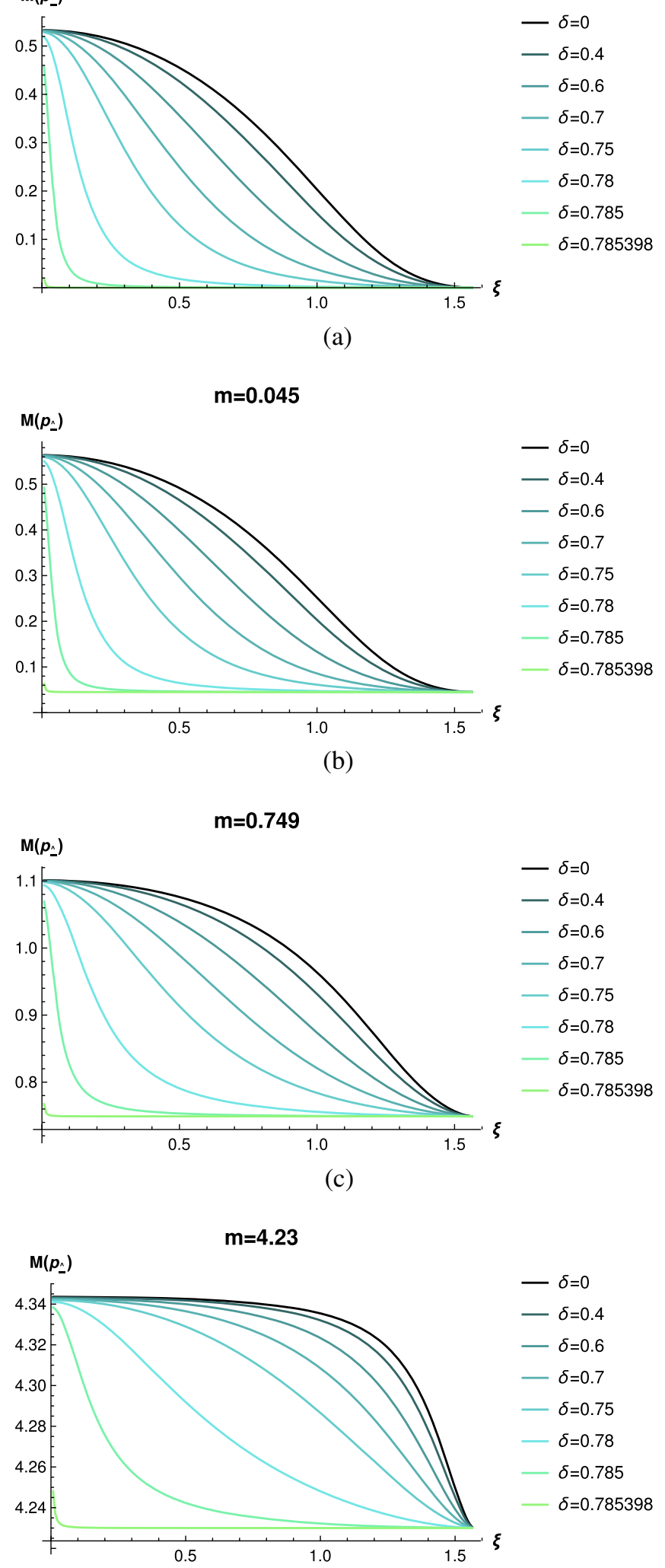

(d)

FIG. 9. Constituent mass as a function of $\xi=\tan ^{-1} p_{\hat{\alpha}}$ for (a) $m=0$, (b) $m=0.045$, (c) $m=0.749$, and (d) $m=4.23$. All quantities are in proper units of $\sqrt{2 \lambda}$. $\mathrm{m}=\mathbf{0 . 1 8}$

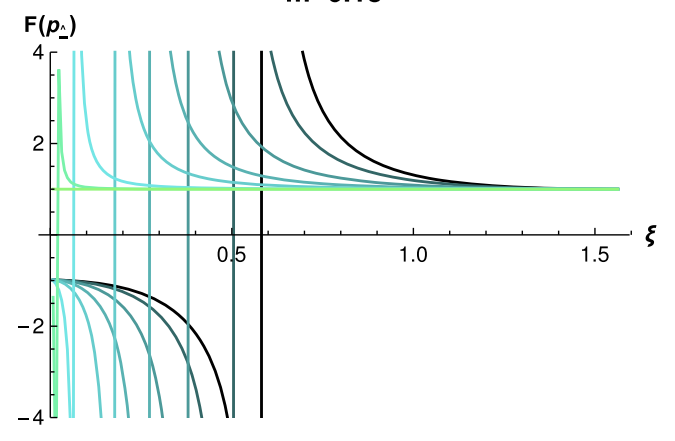

$-\delta=0$
$-\delta=0.4$
$-\delta=0.6$
$-\delta=0.7$
$-\delta=0.75$
$-\delta=0.78$
$-\delta=0.785$
$-\delta=0.785398$

(a)

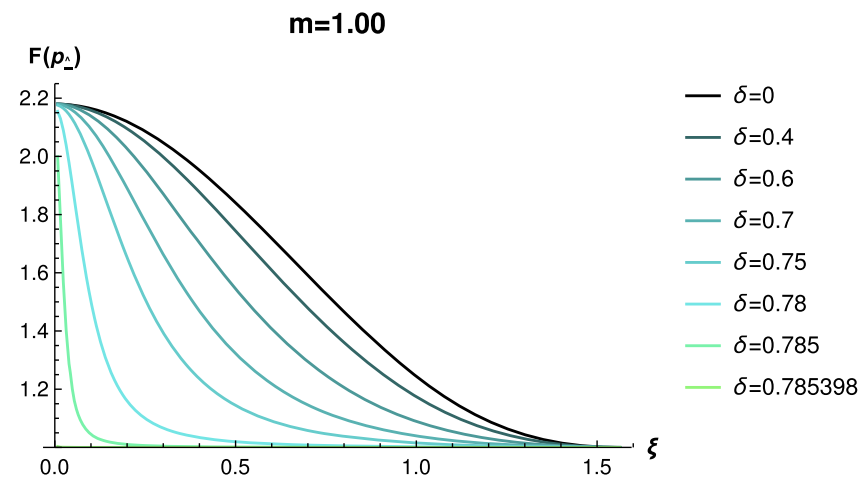

(b)

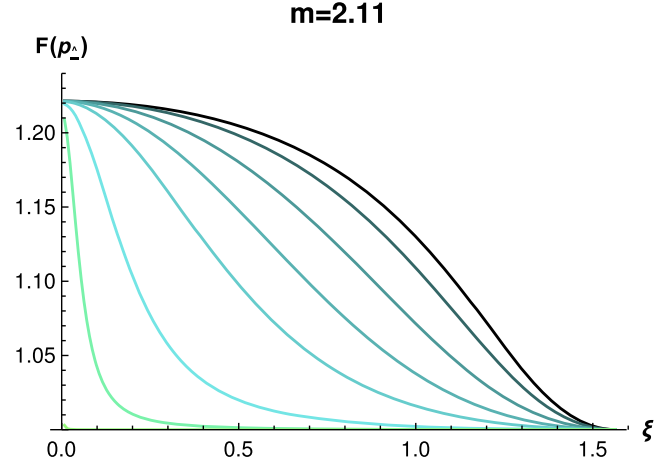

$-\delta=0$

$-\delta=0.4$

$-\delta=0.6$

$-\delta=0.7$

$-\delta=0.75$

$-\delta=0.78$

$\delta=0.785$

$\delta=0.785398$

(c)

FIG. 10. Wave function renormalization as a function of $\xi=\tan ^{-1} p_{\text {合 }}$ for (a) $m=0.18$, (b) $m=1.0$, and (c) $m=2.11$. All quantities are in proper units of $\sqrt{2 \lambda}$.

$$
\cos \theta\left(p_{\hat{\iota}}\right)=\frac{\sqrt{\mathbb{C}} M\left(p_{\hat{\iota}}\right)}{F\left(p_{\hat{\lrcorner}}\right) E\left(p_{\hat{\iota}}\right)},
$$

and

$$
\sin \theta\left(p_{\hat{\iota}}\right)=\frac{p_{\hat{\iota}}}{F\left(p_{\hat{\iota}}\right) E\left(p_{\hat{\iota}}\right)},
$$

so that we may rewrite Eq. (62) associated with the triangle diagram shown in Fig. 2 as 


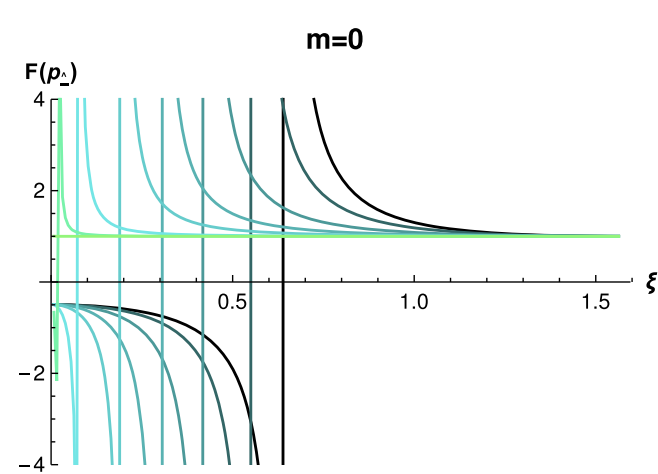

(a)

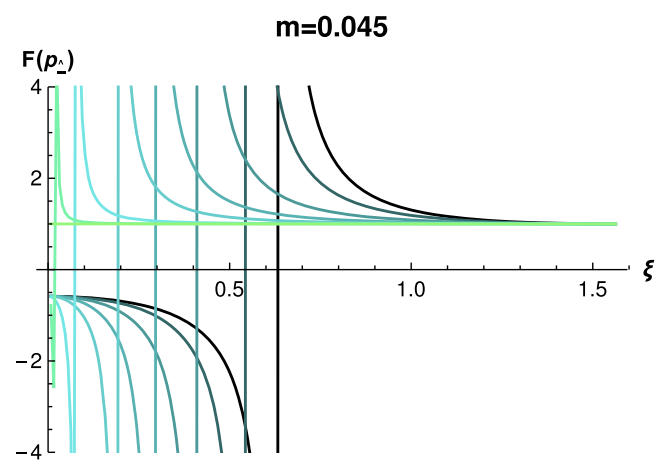

(b)

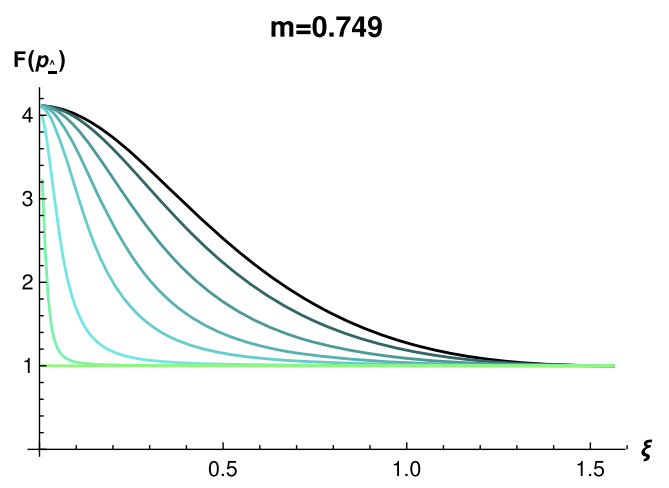

(c)

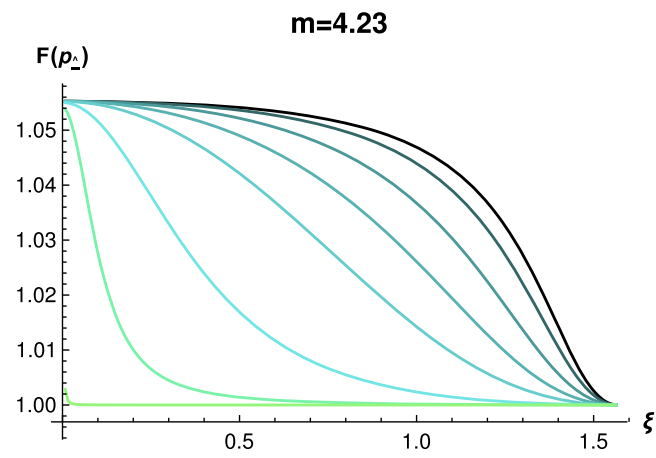

(d)

FIG. 11. Wave function renormalization as a function of $\xi=$ $\tan ^{-1} p_{\text {^ }}$ for (a) $m=0$, (b) $m=0.045$, (c) $m=0.749$, and (d) $m=4.23$. All quantities are in proper units of $\sqrt{2 \lambda}$.

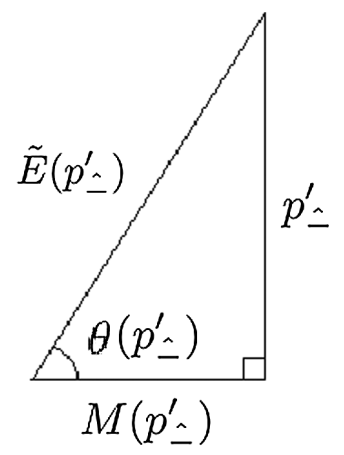

FIG. 12. Geometrical representation of mass gap equations with the interpolation angle independent energy function $\tilde{E}\left(p_{\hat{A}}^{\prime}\right)$ defined in Eq. (95).

$$
F\left(p_{\hat{\sim}}\right) E\left(p_{\hat{\sim}}\right)=\sqrt{\mathbb{C} M\left(p_{\hat{\iota}}\right)^{2}+p_{\hat{\iota}}^{2}},
$$

or

$$
E\left(p_{\hat{\iota}}\right)=\frac{\sqrt{\mathbb{C} M\left(p_{\hat{\iota}}\right)^{2}+p_{\hat{\varkappa}}^{2}}}{F\left(p_{\hat{\imath}}\right)} .
$$

In contrast to Eq. (62), we can now express $E\left(p_{\hat{\Delta}}\right)$ itself without squaring it as $E\left(p_{\hat{\varkappa}}\right)^{2}$ with the support from the wave function renormalization factor $F\left(p_{\hat{\iota}}\right)$ as well as the mass function $M\left(p_{\hat{\sim}}\right)$. This is rather remarkable because the issue of $E\left(p_{\hat{\sim}}\right)$ not being always positive for $m \lesssim 0.56$, which was discussed in Sec. III, is now resolved by expressing the dressed quark propagator $S(p)$ in terms of $F\left(p_{\hat{\iota}}\right)$ and $M\left(p_{\hat{\iota}}\right)$ as given by Eq. (88). While $E\left(p_{\hat{\iota}}\right)$ can be negative, $F\left(p_{\hat{\Lambda}}\right) E\left(p_{\hat{\Lambda}}\right)$ is always positive due to the sign correlation between $E\left(p_{\hat{\epsilon}}\right)$ and $F\left(p_{\hat{\epsilon}}\right)$ as one can see from Eq. (90) or equivalently from Eq. (92) due to the sign correlation between $\theta\left(p_{\hat{\iota}}\right)$ and $p_{\hat{\iota}}$. This allows us more physically transparent interpretation of the energymomentum dispersion relation for the interpolating dressed quark with its self-energy. Moreover, using the rescaled variable $p_{\hat{\iota}}^{\prime}=p_{\iota} / \sqrt{\mathbb{C}}$ introduced in Eq. (83) for the renormalized chiral condensate, we can assert the interpolation angle independence of the rescaled energymomentum dispersion given by

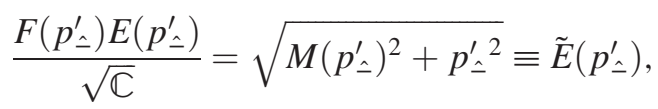

where we define the interpolation angle independent energy function $\tilde{E}\left(p_{\hat{\prime}}^{\prime}\right)$ which extends the interpolating energymomentum dispersion relation of the on-mass-shell particle given by Eq. (7) to the case of the dressed quark with the rescaled variable, i.e.,

$$
\tilde{E}\left(p_{\hat{\sim}}^{\prime}\right)^{2}=p_{\hat{\underline{\Lambda}}}^{\prime 2}+M\left(p_{\hat{\sim}}^{\prime}\right)^{2} .
$$

As the solution of $\tilde{E}\left(p_{\hat{\sim}}^{\prime}\right)$ is always positive in contrast to $E\left(p_{\hat{\wedge}}\right)$, we can now promote the mere pictorial device of 


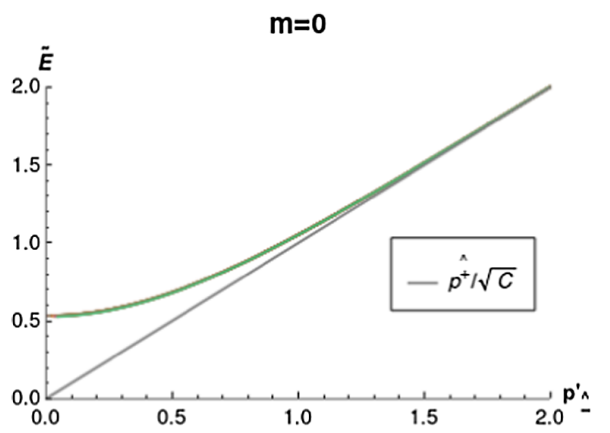

(a)

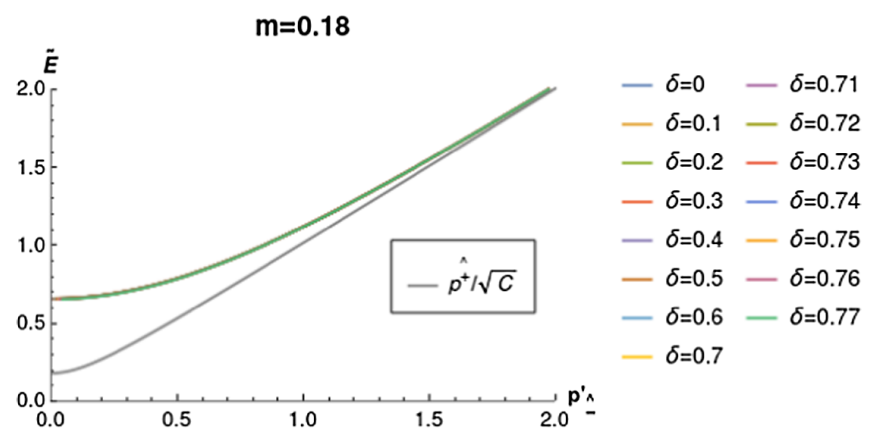

(b)

FIG. 13. The profiles of $\tilde{E}\left(p_{\hat{\varkappa}}^{\prime}\right)$ for several interpolation angles for two example small quark masses (a) $m=0$ and (b) $m=0.18$, in comparison with $p^{\hat{+}} / \sqrt{\mathbb{C}}$ for a free particle of the corresponding same masses, as a function of $p_{\hat{\wedge}}^{\prime}$. All quantities are in proper units of $\sqrt{2 \lambda}$.

geometric interpretation depicted in Fig. 2 to the more physically meaningful geometric interpretation with $\tilde{E}\left(p_{\wedge}^{\prime}\right), M\left(p_{\wedge}^{\prime}\right)$ and $p_{\wedge}^{\prime}$ as shown in Fig. 12 with all the positive lengths of the triangle sides. From Eqs. (7) and (96), we also note the correspondence $m \leftrightarrow M\left(p_{\wedge}^{\prime}\right)$ and $p^{\hat{+}} / \sqrt{\mathbb{C}} \leftrightarrow \tilde{E}\left(p_{\wedge}^{\prime}\right)$ between the bare quark and the dressed quark. As an illustration of this correspondence, we plot the profiles of $\tilde{E}$ as a function of $p_{\wedge}^{\prime}$ for the two cases of $m=0$ and $m=0.18$ in Fig. 13. It is evident that $\tilde{E}\left(p_{\wedge}^{\prime}\right) \rightarrow$ $p^{\hat{+}} / \sqrt{\mathbb{C}}$ as $p_{\wedge}^{\prime} \rightarrow \infty$, which is consistent with the result that the mass function $M\left(p_{\lrcorner}\right)$approaches the bare quark mass value $m$ as $\xi \rightarrow \pi / 2$ or $p_{\wedge} \rightarrow \infty$ (See Figs. 8 and 9). As $p_{\hat{\wedge}}^{\prime} \rightarrow 0$, however, $\widetilde{E}\left(p_{\hat{\lrcorner}}^{\prime}\right)$ approaches the characteristic mass value $M(0)$ as shown in Fig. 13. Indeed we note $\tilde{E}(0)=\frac{F(0) E(0)}{\sqrt{\mathbb{C}}}=M(0)$, confirming the sign correlation between $E\left(p_{\hat{\iota}}\right)$ and $F\left(p_{\hat{\iota}}\right)$ mentioned earlier, i.e., the negativity of $E\left(p_{\hat{\wedge}}\right)$ for the small $p_{\hat{\wedge}}$ region is compensated by the corresponding negativity of $F\left(p_{\hat{\prime}}\right)$ to yield the mass function $M\left(p_{\hat{\prime}}\right)$ positive always for any kinematic region of $p_{\hat{\imath}}$. In Table II, the numerical values of $M(0)$ and $F(0)$ are tabulated for the quark mass values shown in Figs. 8 and 9 as well as in Figs. 10 and 11. As expected, $F(0)$ values are negative for the small bare quark mass values $(m \lesssim 0.56)$ to compensate the corresponding negative values of $E(0)$ while $M(0)$ values are all positive.

Now, between the two limits $\left(p^{\prime}{ }_{\hat{\varkappa}} \rightarrow 0\right.$ and $\left.p_{\hat{\wedge}}^{\prime} \rightarrow \infty\right)$, both $\tilde{E}\left(p_{\hat{\prime}}^{\prime}\right)$ and $M\left(p_{\hat{\prime}}^{\prime}\right)$ are running with the variable $p_{\hat{\Lambda}}^{\prime}$ and their profiles of the $p_{\wedge}^{\prime}$-dependence are completely independent of the interpolation angle $\delta$. The invariance of $\tilde{E}\left(p_{\wedge}^{\prime}\right)$ and $M\left(p_{\wedge}^{\prime}\right)$ under the interpolation between IFD and LFD indicates their universal nature as physically meaningful quantities. In this respect, one may call $\tilde{E}\left(p_{\lrcorner}^{\prime}\right)$ and $M\left(p_{\wedge}^{\prime}\right)$ as the effective energy and the constituent mass of the dressed quark moving with the scaled longitudinal momentum $p_{\star}^{\prime}$. In principle, these physical quantities can be also computed in the well-known Euclidean approaches [32] such as the lattice QCD [33] and the manifestly covariant Dyson-Schwinger formulation. In the Euclidean formulation, which can in principle be applied to the interpolation dynamics as far as $0 \leq \delta<\pi / 4$ except $\delta=\pi / 4$ due to the light-like nature of LFD, the effective energy and the constituent quark mass would be given in terms of the Lorentz-invariant Euclidean variable $\tilde{P}^{2}<0$, i.e., $\tilde{E}\left(\tilde{P}^{2}\right)$ and $M\left(\tilde{P}^{2}\right)$. In IFD, one may correspond the Wick rotated energy $\tilde{P}^{0}$ with the imaginary effective energy, i.e., $\tilde{P}^{0}=i \tilde{E}\left(\tilde{P}^{2}\right)$ (purely imaginary value), and the longitudinal momentum $\tilde{P}^{1}$ with $p^{1}$ of the dressed quark, so that $\tilde{P}^{2}=\left(\tilde{P}^{0}\right)^{2}+$ $\left(\tilde{P}^{1}\right)^{2}=-\tilde{E}\left(\tilde{P}^{2}\right)^{2}+\left(p^{1}\right)^{2}=-\left(p^{1}\right)^{2}-M\left(\tilde{P}^{2}\right)^{2}+\left(p^{1}\right)^{2}=$ $-M\left(\tilde{P}^{2}\right)^{2}<0$. In the same token, as $p^{2}=\frac{\left(p^{+}\right)^{2}-\left(p_{\Perp}\right)^{2}}{\mathbb{C}}$ generally in the interpolating dynamics, the Wick rotated interpolating energy $\tilde{P}^{\hat{+}}$ with the imaginary effective energy, i.e., $\tilde{P}^{\hat{+}} / \sqrt{\mathbb{C}}=i \tilde{E}\left(p_{\hat{\varkappa}}^{\prime}{ }^{2}\right)$ (purely imaginary value), and the longitudinal momentum $\tilde{P}_{\hat{\iota}} / \sqrt{\mathbb{C}}$ with $p_{\wedge}^{\prime}$ of the dressed

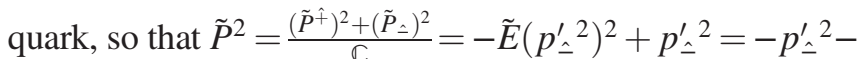
$M\left(p_{\hat{\Lambda}}^{\prime}\right)^{2}+p_{\hat{\Lambda}}^{\prime}{ }^{2}=-M\left(p_{\wedge}^{\prime}\right)^{2}<0$. Thus, it is natural to correspond the square of the rescaled longitudinal momentum $\left(p_{\wedge}^{\prime}\right)$ to the Euclidean variable $\tilde{P}^{2}$ for the space-like region $\tilde{P}^{2}<0$ in the interpolating dynamics, i.e.,

$$
p_{\hat{\Lambda}}^{\prime 2} \leftrightarrow-\tilde{P}^{2} .
$$

TABLE II. The numerical values of $M(0)$ and $F(0)$ for several different quark mass values. All quantities are in proper units of $\sqrt{2 \lambda}$.

\begin{tabular}{lrrrrrrr}
\hline \hline$m$ & 0 & 0.045 & 0.18 & 0.749 & 1.00 & 2.11 & 4.23 \\
\hline$M(0)$ & 0.532778 & 0.563644 & 0.659112 & 1.10105 & 1.31167 & 2.30969 & 4.34358 \\
$F(0)$ & -0.495173 & -0.584175 & -0.987673 & 4.11079 & 2.17976 & 1.22134 & 1.05526 \\
\hline \hline
\end{tabular}


This correspondence is supported not only from the relativistic form invariance of $\tilde{E}\left(p_{\wedge}^{\prime}\right)$ and $M\left(p_{\wedge}^{\prime}\right)$ but also from the matching condition between the Minkowsky space and the Euclidean space. Namely, when the real energy value in the Minkowsky space is converted into the purely imaginary value in the Euclidean space, the matching between the real value and the purely imaginary value occurs precisely where the value itself is zero. For instance, in IFD, the Wick rotation of the energy $p^{0} \rightarrow \tilde{P}^{0}=i p^{0}$ has the common energy value $p^{0}=\widetilde{P}^{0}=0$ so that the Lorentzinvariant momentum-squared value in the Minkowsky space can be matched with the corresponding Euclidean momentum-squared value, $\tilde{P}^{2}$, by taking simultaneously both the real value $p^{0}=0$ in the Minkowsky space and the purely imaginary value $\tilde{P}^{0}=i p^{0}=0$. Likewise, in the interpolation of the relativistic dynamics for $0 \leq \delta<\pi / 4$, one can match the Minkowsky space and the Euclidean space by taking $p^{\hat{+}} / \sqrt{\mathbb{C}}=i p^{\hat{+}} / \sqrt{\mathbb{C}}=\tilde{P}^{\hat{+}} / \sqrt{\mathbb{C}}=0$, confirming the correspondence given by Eq. (97).

\section{THE BOUND-STATE EQUATION}

Having solved the mass gap equation and obtained the dressed quark propagator interpolating between IFD and LFD, we now derive the quark-antiquark bound-state equation in the interpolating form. While we consider the quark-antiquark bound-state with the equal bare mass $m$ in this work, one may generalize it rather straightforwardly to the unequal quark and antiquark mass cases. Bound-states for the unequal quark and antiquark mass cases were analyzed in Refs. [1,34], and the quark-hadron

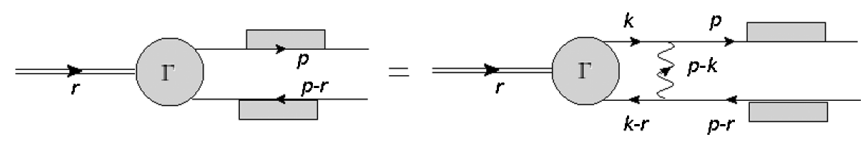

FIG. 14. Diagrammatic representation of the Bethe-Salpeter Equation.

duality, analytical heavy quark expansion and chiral symmetry breaking effects in the heavy-light mesons have been discussed respectively in Refs. [35-37]. Denoting the covariant Bethe-Salpeter amplitude $\Gamma(r, p)$ of the twobody bound state with the two-momentum $r^{\hat{\mu}}$ of the bound state and the two-momentum $p^{\hat{\mu}}$ of one of the two constituents depicted in Fig. 14, we first write down the Bethe-Salpeter equation following the Feynman rules of the gluon propagator and the dressed quark propagator in the 't Hooft model

$\Gamma(r, p)=\frac{i \lambda}{2 \pi} f \frac{d k_{\llcorner} d k_{\hat{+}}}{\left(p_{\varkappa}-k_{\lrcorner}\right)^{2}} S(p) \gamma^{\hat{+}} \Gamma(r, k) \gamma^{\hat{+}} S(p-r)$.

To project the covariant Bethe-Salpeter amplitude $\Gamma(r, p)$ into the interpolating equal-time wave function, we first integrate out the interpolating energy $p_{\hat{\gamma}}$ and define the wave function $\phi\left(r_{\hat{\iota}}, p_{\hat{\iota}}\right)$ as $\phi\left(r_{\hat{\iota}}, p_{\hat{\iota}}\right)=\int d p_{\hat{+}} \Gamma(r, p)$ with the on-mass-shell condition to fix the external interpolating energy $r_{\hat{+}}$. Then, Eq. (98) is converted to the equation for $\phi\left(r_{\hat{\varkappa}}, p_{\hat{\varkappa}}\right)$

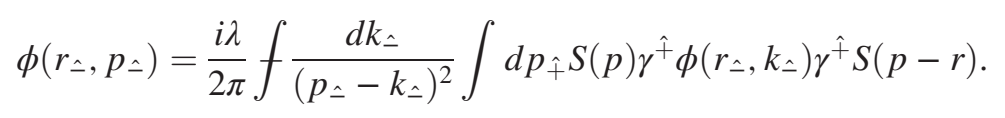

Now, we also write the fermion propagator $S(p)$ given by Eq. (87) as

$$
S(p)=\frac{\tilde{T}\left(p_{\hat{\iota}}\right) \Lambda^{+} \tilde{T}^{\dagger}\left(p_{\hat{\iota}}\right) \gamma^{0}}{p_{\hat{+}}-E_{u}\left(p_{\hat{\iota}}\right)+i \epsilon}+\frac{\tilde{T}\left(p_{\hat{\iota}}\right) \Lambda^{-} \tilde{T}^{\dagger}\left(p_{\hat{\iota}}\right) \gamma^{0}}{p_{\hat{+}}-E_{v}\left(p_{\hat{\iota}}\right)-i \epsilon},
$$

using Eqs. (A21) and (A22) to rewrite $u\left(p_{\hat{\iota}}\right) \bar{u}\left(p_{\hat{\iota}}\right)$ and $v\left(-p_{\hat{\iota}}\right) \bar{v}\left(-p_{\hat{\iota}}\right)$ as

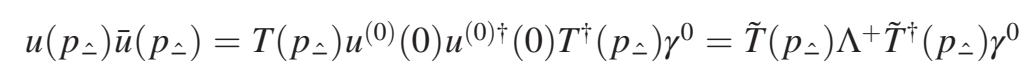

and

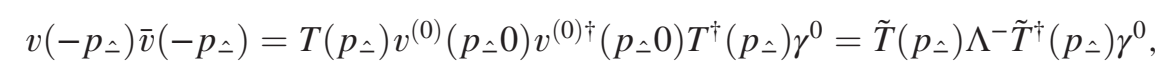

where we define

$$
\Lambda^{+} \equiv \frac{u^{(0)}(0) u^{(0) \dagger}(0)}{2 \sqrt{\mathbb{C}} m}=\left(\begin{array}{cc}
\frac{1}{2(\cos \delta-\sin \delta)} & \frac{1}{2 \sqrt{\complement}} \\
\frac{1}{2 \sqrt{\complement}} & \frac{1}{2(\cos \delta+\sin \delta)}
\end{array}\right)
$$




$$
\Lambda^{-} \equiv \frac{v^{(0)}(0) v^{(0) \dagger}(0)}{2 \sqrt{\mathbb{C}} m}=\left(\begin{array}{cc}
\frac{1}{2(\cos \delta-\sin \delta)} & -\frac{1}{2 \sqrt{\mathbb{C}}} \\
-\frac{1}{2 \sqrt{\mathbb{C}}} & \frac{1}{2(\cos \delta+\sin \delta)}
\end{array}\right)
$$

and

$$
\tilde{T}\left(p_{\hat{\prime}}\right)=\exp \left[-\frac{1}{2} \theta\left(p_{\hat{\prime}}\right) \frac{\gamma_{\hat{\varkappa}}}{\sqrt{\mathbb{C}}}\right]
$$

Substituting then Eq. (100) to Eq. (99) and performing the $p_{\hat{+}}$ integration, we get

$$
\begin{aligned}
& \phi\left(r_{\hat{\iota}}, p_{\hat{\iota}}\right)=\lambda f \frac{d k_{\hat{\varkappa}}}{\left(p_{\hat{\imath}}-k_{\hat{\iota}}\right)^{2}}
\end{aligned}
$$

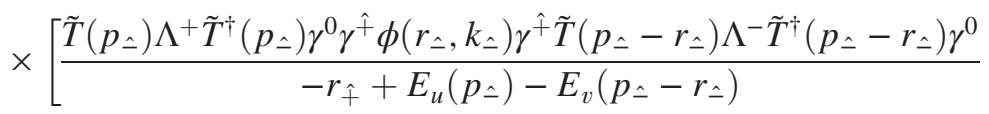

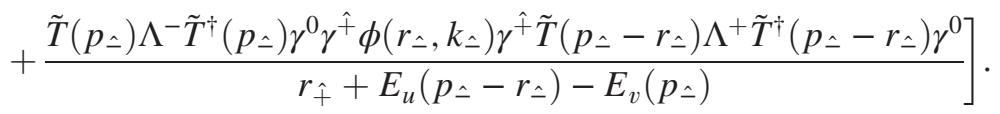

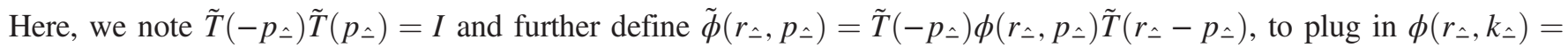
$\tilde{T}\left(k_{\hat{\iota}}\right) \tilde{\phi}\left(r_{\hat{\iota}}, k_{\hat{\varkappa}}\right) \tilde{T}\left(k_{\hat{\iota}}-r_{\hat{\iota}}\right)$ and obtain

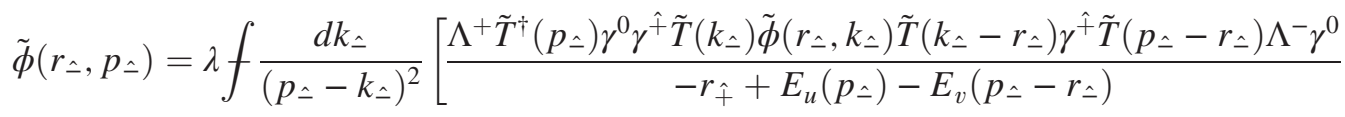

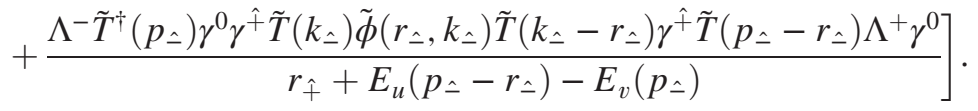

Examining the general structure of $\tilde{\phi}$ in Eq. (107), we realize that it can be split into the forward moving part $\hat{\phi}_{+}$and the backward moving part $\hat{\phi}_{-}$with the two $2 \times 2$ matrices $\hat{M}^{+}$and $\hat{M}^{-}$, i.e., $\tilde{\phi}=\hat{\phi}_{+} \hat{M}^{+}+\hat{\phi}_{-} \hat{M}^{-}$, where

$$
\begin{aligned}
& \hat{M}^{+}=\frac{\gamma^{5} \sqrt{\mathbb{C}}+\gamma_{\hat{\varkappa}}}{2}=\left(\begin{array}{cc}
-\frac{\sqrt{\mathbb{C}}}{2} & \frac{\cos \delta+\sin \delta}{2} \\
-\frac{\cos \delta-\sin \delta}{2} & \frac{\sqrt{\mathbb{C}}}{2}
\end{array}\right), \\
& \hat{M}^{-}=\frac{\gamma^{5} \sqrt{\mathbb{C}}-\gamma_{\hat{\epsilon}}}{2}=\left(\begin{array}{cc}
-\frac{\sqrt{\mathbb{C}}}{2} & -\frac{\cos \delta+\sin \delta}{2} \\
\frac{\cos \delta-\sin \delta}{2} & \frac{\sqrt{\mathbb{C}}}{2}
\end{array}\right) .
\end{aligned}
$$

We note here that $\hat{M}^{+}$and $\hat{M}^{-}$coincide with the two $2 \times 2$ matrices $M^{+}$and $M^{-}$in the IFD given by Eq. (4.9) of Ref. [10] as $\delta \rightarrow 0$ or $\mathbb{C} \rightarrow 1$, i.e., $M^{+}=\frac{\gamma^{5}+\gamma^{1}}{2}=\frac{1+\gamma^{0}}{2} \gamma^{5}$, and $M^{-}=\frac{\gamma^{5}-\gamma^{1}}{2}=\frac{1-\gamma^{0}}{2} \gamma^{5}$, due to $\gamma^{0} \gamma^{5}=\gamma^{1}$, while $\hat{M}^{ \pm} \rightarrow M_{\mathrm{LF}}^{ \pm}= \pm \frac{\gamma^{+}}{2}$ in the LFD limit $\delta \rightarrow \pi / 4$ or $\mathbb{C} \rightarrow 0$. For the interpolation angle $0 \leq \delta \leq \pi / 4$ in general, from the direct calculation of the matrix multiplications, we find

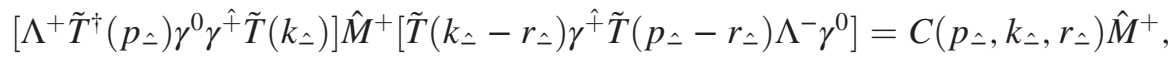

$$
\begin{aligned}
& {\left[\Lambda^{+} \tilde{T}^{\dagger}\left(p_{\hat{\iota}}\right) \gamma^{0} \gamma^{\hat{+}} \tilde{T}\left(k_{\hat{\iota}}\right)\right] \hat{M}^{-}\left[\tilde{T}\left(k_{\hat{\iota}}-r_{\hat{\iota}}\right) \gamma^{\hat{+}} \tilde{T}\left(p_{\hat{\iota}}-r_{\hat{\iota}}\right) \Lambda^{-} \gamma^{0}\right]=-S\left(p_{\hat{\iota}}, k_{\hat{\iota}}, r_{\hat{\iota}}\right) \hat{M}^{+},}
\end{aligned}
$$

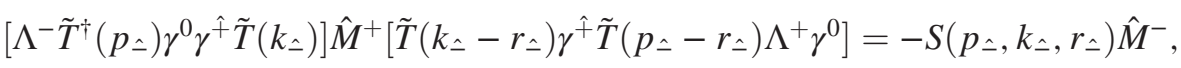

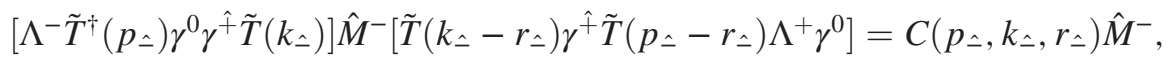

where 


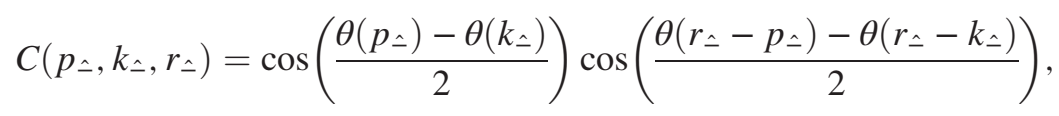

and

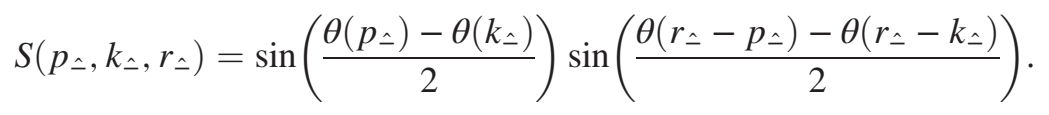

With Eqs. (110)-(113), we finally split Eq. (107) into the two coupled bound-state equations of $\hat{\phi}_{+}$and $\hat{\phi}_{\text {今 }}$

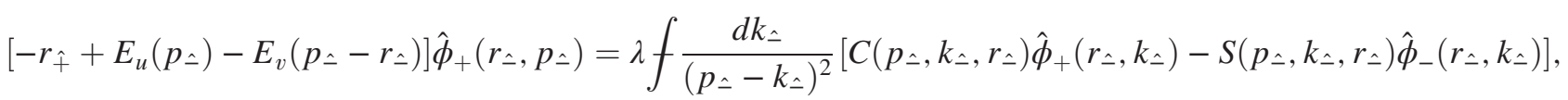

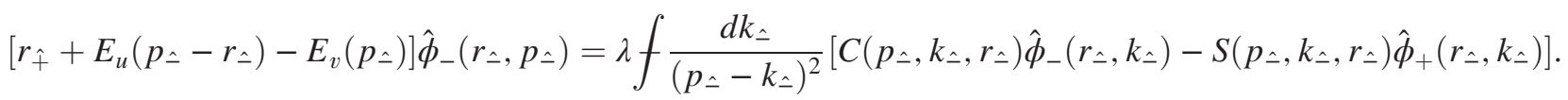

We again note here that Eqs. (116a)-(116b) coincide with Eq. (4.12) of Ref. [10] in the IFD as $\delta \rightarrow 0$ because not only the energies of particle and antiparticle become $E_{u}\left(p_{\lrcorner}\right) \stackrel{\delta \rightarrow 0}{\rightarrow} E\left(p^{1}\right)$ and $E_{v}\left(p_{\lrcorner}\right) \stackrel{\delta \rightarrow 0}{\rightarrow}-E\left(p^{1}\right)$ but also the rest of the variables correspond to their IFD counterparts, e.g., $r_{\hat{+}} \stackrel{\delta \rightarrow 0}{\rightarrow} r^{0}$, etc..

In the LFD limit $\mathbb{C} \rightarrow 0$ (or $\delta \rightarrow \pi / 4$ ) on the other hand, as discussed earlier in Sec. II C, $E_{u}\left(p_{\hat{\iota}}\right) \stackrel{\delta \rightarrow \pi / 4}{\rightarrow} B\left(p^{+}\right)+$ $\frac{m^{2}}{2 p^{+}}=\frac{m^{2}-2 \lambda}{2 p^{+}}$and $E_{v}\left(p_{\iota}-r_{\lrcorner}\right) \stackrel{\delta \rightarrow \pi / 4}{\rightarrow}-B\left(r^{+}-p^{+}\right)-\frac{m^{2}}{2\left(r^{+}-p^{+}\right)}=-\frac{m^{2}-2 \lambda}{2\left(r^{+}-p^{+}\right)}$using Eq. (77) while $E_{u}\left(p_{\lrcorner}-r_{\lrcorner}\right) \stackrel{\delta \rightarrow \pi / 4}{\rightarrow} \frac{2\left(r^{+}-p^{+}\right)}{\mathbb{C}}+$ $B\left(r^{+}-p^{+}\right)+\frac{m^{2}}{2\left(r^{+}-p^{+}\right)}$and $E_{v}\left(p_{\iota}\right) \stackrel{\delta \rightarrow \pi / 4}{\rightarrow}-\frac{2 p^{+}}{\mathbb{C}}$ for the bound-state kinematics $0<p^{+}<r^{+}$. In this limit then, noting $C\left(p^{+}, k^{+}, r^{+}\right) \rightarrow 1$ and $S\left(p^{+}, k^{+}, r^{+}\right) \rightarrow 0$ from Eq. (67), we note that Eq. (116a) gets reduced to

$$
\left[-r^{-}+\frac{m^{2}-2 \lambda}{2 p^{+}}+\frac{m^{2}-2 \lambda}{2\left(r^{+}-p^{+}\right)}\right] \phi\left(r^{+}, p^{+}\right)=\lambda f \frac{d k^{+}}{\left(p^{+}-k^{+}\right)^{2}} \phi\left(r^{+}, k^{+}\right),
$$

where $\phi\left(r^{+}, p^{+}\right)$corresponds to $\hat{\phi}_{+}\left(r_{\lrcorner}, p_{\lrcorner}\right)$in the LFD limit. Also, a solution of zero for $\hat{\phi}_{-}$can be attained rather immediately from Eq. (116b) by dividing it by the energy denominator factor $\left[r_{\hat{+}}+E_{u}\left(p_{\hat{\iota}}-r_{\hat{\iota}}\right)-E_{v}\left(p_{\hat{\iota}}\right)\right]$ and noting the correspondence $1 /\left(r_{\hat{+}}+E_{u}\left(p_{\hat{\iota}}-r_{\hat{\iota}}\right)-E_{v}\left(p_{\hat{\iota}}\right)\right) \rightarrow \mathbb{C} /\left(2 r^{+}+\mathbb{C}\left(r^{-}+B\left(r^{+}-p^{+}\right)+B\left(p^{+}\right)+\frac{m^{2}}{2\left(r^{+}-p^{+}\right)}+\frac{m^{2}}{2\left(p^{+}\right)}\right) \rightarrow 0\right.$ as $\mathbb{C} \rightarrow 0$. Substituting the on-mass-shell condition $r^{-}=\mathcal{M}^{2} /\left(2 r^{+}\right)$for the bound-state meson of mass $\mathcal{M}$, defining the manifestly boost-invariant light-front momentum fraction variables $x=\frac{p^{+}}{r^{+}}(0 \leq x \leq 1)$ and $y=\frac{k^{+}}{r^{+}}(0 \leq y \leq 1)$ and multiplying both sides of the equation by $\left(-2 r^{+}\right)$, we obtain

$$
\left[\mathcal{M}^{2}-\frac{m^{2}-2 \lambda}{x}-\frac{m^{2}-2 \lambda}{1-x}\right] \phi(x)=-2 \lambda f_{0}^{1} \frac{d y}{(x-y)^{2}} \phi(y)
$$

where the $r^{+}$independence of $\phi\left(r^{+}, p^{+}\right)$and $\phi\left(r^{+}, k^{+}\right)$is imposed in $\phi(x)$ and $\phi(y)$ due to the boost-invariance of the light-front bound-state equation correctly reproducing 't Hooft's bound-state equation, Eq. (25) in Ref. [1].

In general, relating $E_{u}$ 's and $E_{v}$ 's in Eq. (116) to the solutions of $E$ in Eq. (65), we summarize the interpolating coupled bound-state equations for $\hat{\phi}_{+}\left(r_{\hat{\iota}}, p_{\iota}\right)$ and $\hat{\phi}_{-}\left(r_{\iota}, p_{\hat{\iota}}\right)$ between IFD and LFD as follows:

$$
\begin{aligned}
& {\left[-r_{\hat{+}}+\frac{-\mathbb{S} p_{\hat{\iota}}+E\left(p_{\hat{\iota}}\right)}{\mathbb{C}}+\frac{\mathbb{S}\left(p_{\hat{\iota}}-r_{\hat{\iota}}\right)+E\left(p_{\hat{\iota}}-r_{\hat{\iota}}\right)}{\mathbb{C}}\right] \hat{\phi}_{+}\left(r_{\hat{\iota}}, p_{\hat{\iota}}\right)}
\end{aligned}
$$

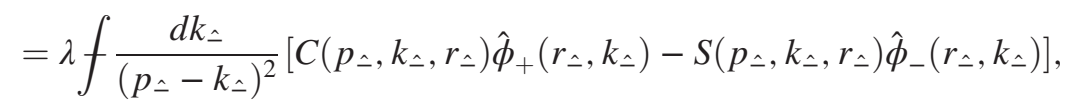




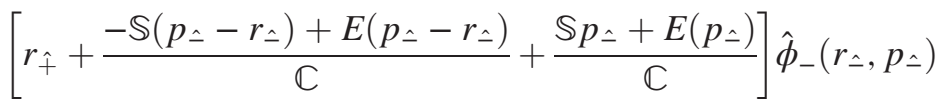

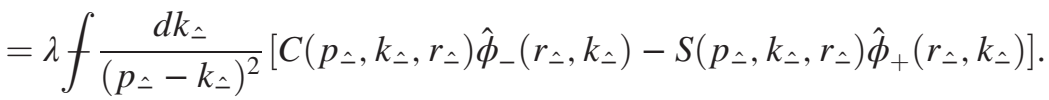

In the next section, Sec. VI, we solve Eqs. (119a)-(119b) using the solutions of Eqs. (48) and (51) to determine the bound-state mass spectrum as well as the corresponding bound-state wave functions $\hat{\phi}_{+}\left(r_{\hat{\iota}}, p_{\hat{\iota}}\right)$ and $\hat{\phi}_{-}\left(r_{\hat{\iota}}, p_{\hat{\iota}}\right)$ interpolating between IFD and LFD.

\section{THE BOUND-STATE SOLUTION}

Solving Eqs. (119a)-(119b) in practice, we use $r^{\hat{+}}=\mathbb{C} r_{\hat{+}}+\mathbb{S} r_{\lrcorner}$and scale the interpolating quark momentum variables $p_{\wedge}$ and $k_{\wedge}$ with respect to the interpolating bound-state momentum $r_{\hat{\imath}}$ introducing the interpolating momentum fraction variables $x=p_{\wedge} / r_{\varkappa}$ and $y=k_{\varkappa} / r_{\hat{\iota}}$, respectively. ${ }^{3}$

Noting the conversion between the upper and lower indices of the momentum variable, we rewrite Eqs. (119a) and (119b) respectively as follows,

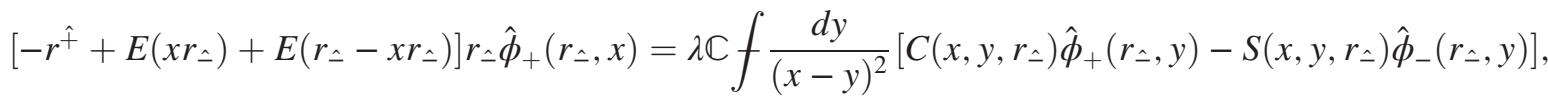

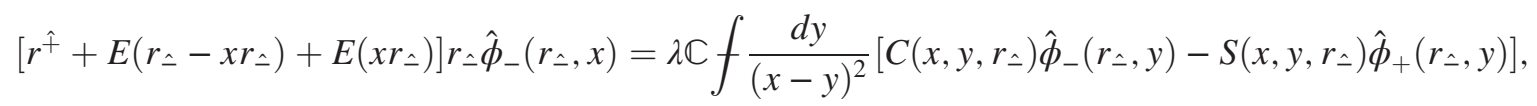

where

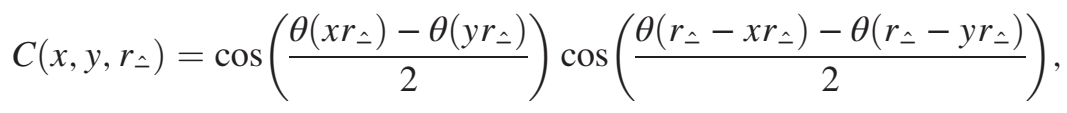

and

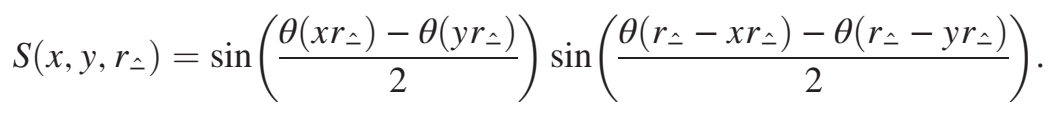

One may note here that the ordinary longitudinal momentum $r^{1}$ is related to the interpolating longitudinal momentum $r_{\wedge}$ as

$$
r^{1}=\frac{1}{\mathbb{C}}\left(r_{\wedge} \cos \delta-\sqrt{r_{\wedge}^{2} \cos ^{2} \delta-\mathbb{C}\left(r_{\wedge}^{2}-\mathcal{M}^{2} \sin ^{2} \delta\right)}\right)
$$

due to the on-mass-shell condition for the meson mass $\mathcal{M}$ as $r^{\hat{\mu}} r_{\hat{\mu}}=r^{\mu} r_{\mu}=\mathcal{M}^{2}$. In this section, we solve the coupled equations Eqs. (120a) and (120b) to find the bound-state mass spectrum $\mathcal{M}_{(n)}^{2}=\frac{\left(r_{(n)}^{+}\right)^{2}-\left(r_{\varkappa}\right)^{2}}{\mathbb{C}}$ and the corresponding wave functions $\hat{\phi}_{ \pm}^{(n)}\left(r_{\lrcorner}, x\right)$ for the $n$th bound state as we discuss in Sec. VI A and Sec. VI B, respectively. Then, we also apply the $\hat{\phi}_{ \pm}^{(n)}\left(r_{\hat{\iota}}, x\right)$ solutions to obtain the interpolating PDFs and discuss the comparison with the quasi-PDFs in Sec. VIC.

\section{A. Spectroscopy}

As discussed in Ref. [30], the bound-state wave functions $\hat{\phi}_{ \pm}^{(n)}\left(r_{\hat{\iota}}, x\right)$ correspond to the coefficients of the compound operators creating/annihilating color singlet quark and antiquark bound states in the generalized Bogoliubov transformation. In contrast to the Bogoliubov transformation for the fermion operators given by Eq. (23) with the coefficients satisfying the normalization $\cos ^{2} \zeta+\sin ^{2} \zeta=1$, the coefficients of the generalized Bogoliubov transformation for the meson states satisfy the normalization condition [12]

\footnotetext{
${ }^{3}$ For the computation with $r_{\imath}=0$ as in the IFD calculation at the meson rest frame [11], we do not scale the interpolating momentum variables but directly take $r_{\hat{\imath}}=0$ in Eqs. (119a)-(119b) as separately presented in Appendix F.
} 


$$
\int d x\left\{\left|\hat{\phi}_{+}^{(n)}\left(r_{\hat{\iota}}, x\right)\right|^{2}-\left|\hat{\phi}_{-}^{(n)}\left(r_{\hat{\sim}}, x\right)\right|^{2}\right\}=1 .
$$

In conjunction with the compound operators [30], it is interesting to note that the scalar or fermionic matter fields transforming in the adjoint representation of $S U(N)$ have been discussed in the literature [38].

In solving numerically the coupled integral equations, Eqs. (120a) and (120b), we use the spectrum method illustrated in Refs. [11,12]. While the rest frame basis wave function [11] was generalized to the moving frame basis wave function [12] in IFD, we further generalize it to the interpolating basis wave function applicable to any interpolation angle $\delta$ between IFD and LFD, i.e.,

$$
\begin{aligned}
& \Psi_{m}\left(\alpha, r_{\hat{\lrcorner}}, x\right) \\
& =\sqrt{\frac{\left|r_{\hat{\lrcorner}}\right| \alpha}{2^{m} m ! \sqrt{\pi}}} \exp \left[-\frac{\alpha^{2} r_{\hat{\lrcorner}}^{2}(1-2 x)^{2}}{8}\right] H_{m}\left(\frac{\alpha r_{\hat{二}}}{2}(1-2 x)\right),
\end{aligned}
$$

where $H_{m}$ is the $m$-th Hermite polynomial and $\alpha$ is the variational parameter which can be tuned to minimize the mass of the ground state. The reason for the Hermite polynomial basis can be traced back to the similarity between the $\lambda=0$ free Hamiltonian expressed in terms of the quark-antiquark compound operators [30] and the simple harmonic oscillator Hamiltonian. Due to the charge conjugation symmetry of the bound states, the wave functions of the mesons $\hat{\phi}_{+}$and $\hat{\phi}_{-}$are then the superpositions of these basis functions

$\hat{\phi}_{ \pm}^{(n)}\left(r_{\hat{\iota}}, x\right)= \begin{cases}\sum_{m=0}^{N-1} a_{m}^{(n) \pm} \Psi_{2 m}\left(\alpha, r_{\hat{\lrcorner}}, x\right), & n \text { even } \\ \sum_{m=0}^{N-1} b_{m}^{(n) \pm} \Psi_{2 m+1}\left(\alpha, r_{\hat{\lrcorner}}, x\right), & n \text { odd }\end{cases}$

where the momentum fraction $x=p_{\text {^ }} / r_{\hat{\wedge}}$ goes to the familiar light-front momentum fraction $p^{+} / r^{+}$as $\delta \rightarrow \pi / 4$. While $x=p_{\text {^ }} / r_{\text {^ }}$ is unbounded, $x \in(-\infty, \infty)$, for $0 \leq \delta<\pi / 4$ in solving the interpolating coupled bound-state equations given by Eqs. (120a)-(120b), $x$ gets bounded to be in $[0,1]$ consistently satisfying the boundstate kinematics $0 \leq p^{+} \leq r^{+}$in LFD at $\delta=\pi / 4$ (or $\mathbb{C}=0$ ) and the two coupled equations, Eqs. (120a) and (120b), get reduced to the single light-front bound-state equation given by Eq. (118). We check this LFD reduction by confirming the correspondence of the interpolating solutions $\hat{\phi}_{+}\left(r_{\hat{\iota}}, p_{\hat{\iota}}\right) \rightarrow \phi(x)$ and $\hat{\phi}_{-}\left(r_{\hat{\iota}}, p_{\hat{\iota}}\right) \rightarrow 0$ in the limit $\delta \rightarrow \pi / 4$ (or $\mathbb{C} \rightarrow 0$ ).

In solving the coupled equations, Eqs. (120a)-(120b), for the ground state $n=0$ as well as the $n$ even excited states, we use the orthonormal basis of $\Psi_{2 m}\left(\alpha, r_{\hat{\wedge}}, x\right)$ with $m=0,1,2, \ldots N-1$ due to the $x \leftrightarrow 1-x$ symmetry. In contrast, for the $n$ odd excited states, we use the orthonormal basis of $\Psi_{2 m+1}\left(\alpha, r_{\wedge}, x\right)$ with $m=$ $0,1,2, \ldots N-1$ due to the $x \leftrightarrow 1-x$ antisymmetry dictated by the charge conjugation symmetry. By projecting Eqs. (120a) and (120b) onto the given set of orthonormal basis functions and integrating over the momentum fraction $x$ on both sides, Eqs. (120a)-(120b) are then transformed into a matrix eigenvalue equation in the orthonormal basis given by Eq. (126) with $m=0,1,2, \ldots N-1$, i.e., $\Psi_{2 m}$ or $\Psi_{2 m+1}$ depending on whether $n$ is even or odd. Due to the coupling between $\hat{\phi}_{+}$and $\hat{\phi}_{-}$, the size of the matrix to be diagonalized is then $2 N \times 2 N$ for either $n$ even or $n$ odd states. While we get $2 N$ of $r_{(n)}^{\hat{+}}$ eigenvalues by diagonalizing the $2 N \times 2 N$ matrix, what we actually find in the meson spectroscopy is just $N$ number of meson masses via $\mathcal{M}_{(n)}^{2}=\frac{\left(r_{(n)}^{+}\right)^{2}-\left(r_{\Perp}\right)^{2}}{\mathbb{C}}$ as the half of the $2 N$ eigenvalues of $r_{(n)}^{+}$ are the same but opposite sign of the other half of the $r_{(n)}^{\hat{+}}$ eigenvalues. For the ground state $n=0$ and the $n$ even excited states, the $N$ number of $\mathcal{M}_{(n)}$ values are found from the lowest one as the ground state meson mass $\mathcal{M}_{(0)}$ to the exited state meson masses $\mathcal{M}_{(2)}, \mathcal{M}_{(4)}$, etc., all the way up to $\mathcal{M}_{2(N-1)}$. For the $n$ odd excited states, the $N$ number of $\mathcal{M}_{(n)}$ values are found from the lowest one as the first excited state meson mass $M_{(1)}$ to the higher exited state meson masses $\mathcal{M}_{(3)}, \mathcal{M}_{(5)}$, etc., all the way up to $\mathcal{M}_{(2 N-1)}$.

In the numerical computation, we note that the numerical accuracy of the results depends not only on the number of basis functions but also the number of grid points for the numerical integrations. In practice, we take $N=20$ in Eq. (126) to get the mass spectrum of the lowest 8 states, i.e., $n=0,1,2, \ldots, 7$, and the number of grid points as 200 for the computation of the integrations in Eqs. (120a) and (120b). As our numerical method involves the values of the interpolation angle $\delta$ and the interpolating longitudinal momentum $r_{\wedge}$, we check the stability of our numerical results and their accuracy by varying $\delta$ and $r_{\hat{\Lambda}}$. While our numerical program provides typically the numerical accuracy of up to 4 or 5 digits for most $\delta$ and $r_{\wedge}$ values with the number of grid points being 200 for the computation of the integrations, we need to pay attention to the accuracy of the numerical values for the mass spectrum when we take $\delta$ close to $\pi / 4$ or $r_{\text {^ }}$ large. Especially, as the meson mass $\mathcal{M}_{(n)}=\sqrt{\frac{\left(r_{(n)}^{+}\right)^{2}-\left(r_{\Perp}\right)^{2}}{\mathbb{C}}}$ gets smaller, the eigenvalues $r_{(n)}^{\hat{+}}$ that we obtain from Eqs. (120a) and (120b) gets closer to the value $r_{-}$that we take for our numerical computation so that it may lead to the cancellation of the two large values in getting the small value. The demand of numerical accuracy gets even more enhanced when $\delta$ gets close to $\pi / 4$ (or $\mathbb{C}$ gets close to zero) and $r_{-}$gets large as both the values in the numerator and the denomination get closer to zero.

In particular, as the bare quark mass $m \rightarrow 0$, we need to check our numerical accuracy paying attention to the GOR 


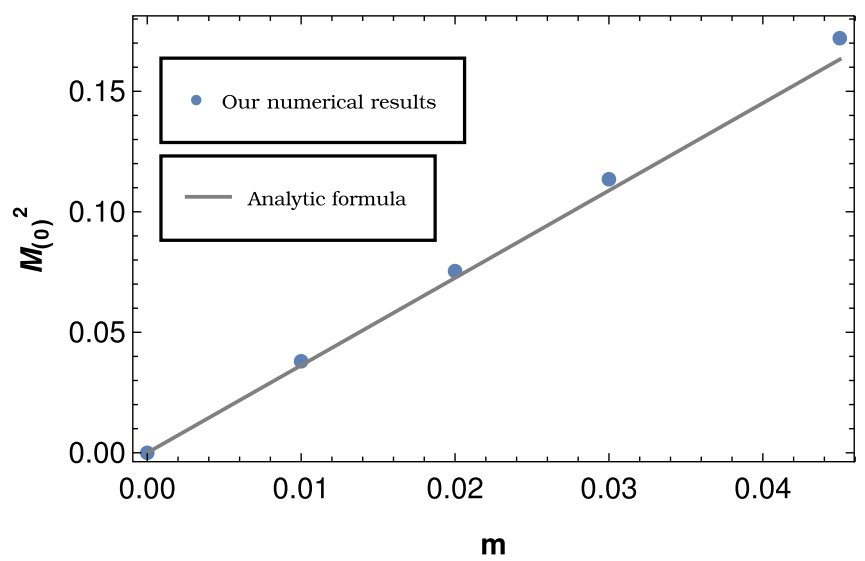

FIG. 15. The bare quark mass $m$ dependence of $\mathcal{M}_{\pi}{ }^{2}$. All quantities are in proper units of $\sqrt{2 \lambda}$. Our numerical results are compared with the analytic result given by Eq. (127).

relation which was explicitly derived in the 't Hooft model [30] and identifying the pionic bound state $\mathcal{M}_{(0)}^{2} \sim$ $m \sqrt{\lambda} \rightarrow 0$. To confirm the validity of GOR relation numerically, we have computed $\mathcal{M}_{(0)}^{2}$ with 600 grid points (rather than 200 grid points normally used in our computation) for the very small $m$ values ( $m=0,0.01,0.02,0.03,0.045$ ) The results are plotted in Fig. 15. As we can see in Fig. 15, we numerically confirm the behavior of $\mathcal{M}_{(0)}^{2} \sim m \sqrt{\lambda}$ and indeed obtain the slope of the straight line consistent with the analytic result given by ${ }^{4}$

$$
\mathcal{M}_{\pi}^{2}=-\frac{4 m\langle\bar{\psi} \psi\rangle}{f_{\pi}^{2}}=\sqrt{\frac{8 \pi^{2} m^{2} \lambda}{3}},
$$

where we used Eq. (79) for the vacuum condensation $\langle\bar{\psi} \psi\rangle$ and the pion decay constant $f_{\pi}=\sqrt{N_{c} / \pi}$ derived [30] from the matrix element of the axial vector current between the pionic ground state and the nontrivial vacuum. Here, the pionic bound state in the chiral limit corresponds to the zero mass bound state that occurs when both quarks have mass zero as pointed out by 't Hooft [1]. The corresponding bound state wave function in LFD is given by $\phi(x)=1$ for $x \in[0,1]$ as we will discuss in the next subsection, Sec. VI B. These results are consistent with the discussions $[4,12,30,37]$ on the SBCS in the 't Hooft model $\left(N_{c} \rightarrow \infty\right)$ which does not contradict with the Coleman's theorem [26] that would prohibit the SBCS in the case of a finite $N_{c}$. As the result of $\mathcal{M}_{(0)}$ must be zero theoretically for the bare quark mass $m=0$, the scrutiny of the numerical sensitivity check depending on the values of $\delta$ and $r_{\wedge}$ as well as the number of computational grid points is maximally enhanced in the $m=0$ case. In Table III, the

\footnotetext{
${ }^{4}$ There is the factor 2 difference between Refs. [30] and $[12,34,39]$ in the coefficient of Eq. (127). Our result is consistent with Refs. [12,34,39].
}

TABLE III. Numerical results of the ground-state meson mass $\mathcal{M}_{(0)}$ depending on the values of $\delta$ and $r_{\hat{\wedge}}$ as well as the number of computational grid points for the quark bare mass $m=0$ case. All the mass spectra are given in six significant figures with the proper unit of $\sqrt{2 \lambda}$. The ground-state meson mass $\mathbf{M}_{0.18}=0.88$ for $m=0.18$ is taken as the reference value of the interpolating longitudinal momentum $r_{\hat{\imath}}$. The results for $r_{\hat{\imath}}=0$ were obtained with the method presented in Appendix F.

\begin{tabular}{lccl}
\hline \hline$\delta$ & $r_{\varkappa}$ & Number of grid points & \multicolumn{1}{c}{$\mathcal{M}_{(0)}$} \\
\hline 0 & 0 & 200 & 0.0607101 \\
& & 600 & 0.0361024 \\
0 & $0.2 \mathbf{M}_{0.18}$ & 200 & 0.0632755 \\
& & 600 & 0.0395562 \\
0 & $2 \mathbf{M}_{0.18}$ & 200 & 0.107216 \\
& & 600 & 0.0532456 \\
0.6 & 0 & 200 & 0.0690707 \\
& & 600 & 0.0407446 \\
0.6 & $0.2 \mathbf{M}_{0.18}$ & 200 & 0.0678997 \\
& & 600 & 0.0472921 \\
0.6 & $2 \mathbf{M}_{0.18}$ & 200 & 0.143686 \\
& & 600 & 0.0599700 \\
\hline \hline
\end{tabular}

numerical values of $\mathcal{M}_{(0)}$ for the bare quark mass $m=0$ are listed depending on the values of $\delta$ and $r_{\lrcorner}$as well as the number of computational grid points. We take the values of $r_{\hat{\imath}}$ as $r_{\hat{-}}=0,0.2 \mathbf{M}_{0.18}$, and $2 \mathbf{M}_{0.18}$, with the scale of $\mathbf{M}_{0.18}$ which is the lowest bound-state mass for $m=0.18$, i.e., $\mathbf{M}_{0.18}=0.88$, as the lowest bound-state mass for $m=0$ is zero, i.e., $\mathbf{M}_{0}=0$, and cannot be taken as any reference value of $r_{\lrcorner}$. While it is highly challenging to achieve the typical numerical accuracy mentioned earlier for the bare quark mass $m=0$, our numerical results appear to consistently approach the theoretical value $\mathcal{M}_{(0)}=0$ as the number of grid points is increased from 200 to 600 for the ranges of the $\delta$ and $r_{\wedge}$ values in Table III. As $\delta$ gets close to $\pi / 4$, our numerical computation demands much higher numerical accuracy. Although we have not increased the number of grid points beyond 600, we anticipate that our numerical results would get closer and closer to zero as we increase the number of grid points even if $\delta$ gets close to $\pi / 4$.

In Table IV, we list the results of the meson mass spectra $\mathcal{M}_{(n)}$ up to $n=7$ for the bare quark mass $m=0$ including $\mathcal{M}_{(0)}$ obtained with the 600 grid points for $\delta=0,0.6$ and 0.78. For the mass spectra of excited states, $\mathcal{M}_{(n)}(n=1,2, \ldots, 7)$, the results were obtained with the number of grid points just 200 for $\delta=0$ and 0.6 although the number of grid points for the $\delta=0.78$ case was still kept as 600. Similarly, we present the results of the meson mass spectra for the bare quark mass $m=0.18$ in Table V. Here, all the results $\mathcal{M}_{(n)}(n=0,1,2, \ldots, 7)$ including the ground-state were obtained with the 200 grid points for $\delta=0$ and 0.6 while for $\delta=0.78$ with the 380 grid points. We note that our numerical results are consistent with each 
TABLE IV. Meson mass spectra for the bare quark mass $m=0$ with the variation of $\delta$ and $r_{\wedge}$ values. All the mass spectra are given in six significant figures with the proper unit of $\sqrt{2 \lambda}$. The ground-state meson mass $\mathbf{M}_{0.18}=0.88$ for $m=0.18$ is taken as the reference value of the interpolating longitudinal momentum $r_{\hat{\imath}}$. The results for $r_{\hat{\imath}}=0$ were obtained with the method presented in Appendix F.

\begin{tabular}{lccccc}
\hline \hline$\delta$ & $r_{\wedge}$ & $\mathcal{M}_{(0)}$ & $\mathcal{M}_{(2)}$ & $\mathcal{M}_{(4)}$ & $\mathcal{M}_{(6)}$ \\
\hline 0 & 0 & 0.0361024 & 3.76245 & 5.68513 & 7.15304 \\
& $0.2 \mathbf{M}_{0.18}$ & 0.0395562 & 3.76266 & 5.68573 & 7.15381 \\
& $2 \mathbf{M}_{0.18}$ & 0.0532456 & 3.76433 & 5.68703 & 7.16921 \\
0.6 & 0 & 0.0407446 & 3.76302 & 5.68552 & 7.15329 \\
& $0.2 \mathbf{M}_{0.18}$ & 0.0472921 & 3.76285 & 5.68542 & 7.15361 \\
& $2 \mathbf{M}_{0.18}$ & 0.0599700 & 3.76441 & 5.68690 & 7.15444 \\
0.78 & 0 & 0.0884992 & 3.76458 & 5.68665 & 7.15417 \\
& $0.2 \mathbf{M}_{0.18}$ & 0.173979 & 3.76292 & 5.69002 & 7.15655 \\
& $2 \mathbf{M}_{0.18}$ & 0.106365 & 3.77182 & 5.69178 & 7.16045 \\
$\delta$ & $r_{А}$ & $\mathcal{M}_{(1)}$ & $\mathcal{M}_{(3)}$ & $\mathcal{M}_{(5)}$ & $\mathcal{M}_{(7)}$ \\
0 & 0 & 2.42728 & 4.80619 & 6.45726 & 7.79124 \\
& $0.2 \mathbf{M}_{0.18}$ & 2.42773 & 4.80668 & 6.45797 & 7.79191 \\
& $2 \mathbf{M}_{0.18}$ & 2.42861 & 4.80603 & 6.45736 & 7.79204 \\
0.6 & 0 & 2.42772 & 4.80668 & 6.45756 & 7.79145 \\
& $0.2 \mathbf{M}_{0.18}$ & 2.42815 & 4.80693 & 6.45796 & 7.79205 \\
& $2 \mathbf{M}_{0.18}$ & 2.43215 & 4.80696 & 6.45845 & 7.79172 \\
0.78 & 0 & 2.42906 & 4.80807 & 6.45857 & 7.79239 \\
& $0.2 \mathbf{M}_{0.18}$ & 2.43734 & 4.80832 & 6.45799 & 7.79376 \\
& $2 \mathbf{M}_{0.18}$ & 2.43520 & 4.81589 & 6.46355 & 7.79905 \\
\hline \hline & & & & &
\end{tabular}

TABLE V. Meson mass spectra for the bare quark mass $m=$ 0.18 with the variation of $\delta$ and $r_{\wedge}$ values. All the mass spectra are given in six significant figures with the proper unit of $\sqrt{2 \lambda}$. The ground-state meson mass $\mathbf{M}_{0.18}=0.88$ for $m=0.18$ is taken as the reference value of the interpolating longitudinal momentum $r_{\wedge}$. The results for $r_{\wedge}=0$ were obtained with the method presented in Appendix F.

\begin{tabular}{lccccc}
\hline \hline$\delta$ & $r_{\text {A }}$ & $\mathcal{M}_{(0)}$ & $\mathcal{M}_{(2)}$ & $\mathcal{M}_{(4)}$ & $\mathcal{M}_{(6)}$ \\
\hline 0 & 0 & 0.880457 & 3.99902 & 5.85781 & 7.29550 \\
& $0.2 \mathbf{M}_{0.18}$ & 0.880686 & 3.99928 & 5.85843 & 7.29630 \\
& $2 \mathbf{M}_{0.18}$ & 0.883080 & 3.99921 & 5.85774 & 7.29676 \\
0.6 & 0 & 0.881753 & 3.99943 & 5.85807 & 7.29566 \\
& $0.2 \mathbf{M}_{0.18}$ & 0.881526 & 3.99943 & 5.85812 & 7.29612 \\
& $2 \mathbf{M}_{0.18}$ & 0.886997 & 4.00048 & 5.85938 & 7.29641 \\
0.78 & 0 & 0.889730 & 4.00233 & 5.86016 & 7.29718 \\
& $0.2 \mathbf{M}_{0.18}$ & 0.856979 & 3.99561 & 5.85482 & 7.29324 \\
& $2 \mathbf{M}_{0.18}$ & 0.914992 & 4.01176 & 5.86721 & 7.30555 \\
$\delta$ & $r_{\text {A }}$ & $\mathcal{M}_{(1)}$ & $\mathcal{M}_{(3)}$ & $\mathcal{M}_{(5)}$ & $\mathcal{M}_{(7)}$ \\
0 & 0 & 2.73527 & 5.00349 & 6.61265 & 7.92348 \\
& $0.2 \mathbf{M}_{0.18}$ & 2.73565 & 5.00401 & 6.61336 & 7.92401 \\
& $2 \mathbf{M}_{0.18}$ & 2.73588 & 5.00338 & 6.61320 & 7.92469 \\
0.6 & 0 & 2.73559 & 5.00384 & 6.61285 & 7.92360 \\
& $0.2 \mathbf{M}_{0.18}$ & 2.73595 & 5.00406 & 6.61329 & 7.92419 \\
& $2 \mathbf{M}_{0.18}$ & 2.73818 & 5.00430 & 6.61360 & 7.92388 \\
0.78 & 0 & 2.73843 & 5.00638 & 6.61463 & 7.92496 \\
& $0.2 \mathbf{M}_{0.18}$ & 2.73213 & 5.00048 & 6.60966 & 7.92062 \\
& $2 \mathbf{M}_{0.18}$ & 2.75685 & 5.01497 & 6.62144 & 7.93055 \\
\hline \hline & & & & &
\end{tabular}

TABLE VI. Summary of meson mass spectra $\mathcal{M}_{(n)}$ $(n=0,1,2, \ldots, 7)$ for the bare quark mass values $m=0$, $0.045,0.18,1.00,2.11$ in the unit of $\sqrt{2 \lambda}$.

\begin{tabular}{lcccccccc}
\hline \hline$n$ & 0 & 1 & 2 & 3 & 4 & 5 & 6 & 7 \\
\hline$m=0$ & 0 & 2.43 & 3.76 & 4.81 & 5.69 & 6.46 & 7.15 & 7.79 \\
$m=0.045$ & 0.42 & 2.50 & 3.82 & 4.85 & 5.73 & 6.49 & 7.19 & 7.82 \\
$m=0.18$ & 0.88 & 2.74 & 4.00 & 5.00 & 5.86 & 6.61 & 7.30 & 7.92 \\
$m=1.00$ & 2.70 & 4.16 & 5.21 & 6.09 & 6.85 & 7.53 & 8.16 & 8.75 \\
$m=2.11$ & 4.91 & 6.17 & 7.06 & 7.83 & 8.51 & 9.13 & 9.69 & 10.23 \\
\hline \hline
\end{tabular}

other up to the second digit after the decimal point for the $\delta$ values not close to $\pi / 4$ such as $\delta=0$ and 0.6 and the $r_{-}$ values not so large such as $r_{\hat{-}}=0$ and $0.2 \mathbf{M}_{0.18}$ as listed in Tables IV and V. Such stability persists in all the cases of the bare quark mass values $(m=0,0.045,0.18,1.00,2.11)$ that we considered for the computation of the meson mass spectra in this work.

In Table VI, we summarize these stable numerical results up to the second digit after the decimal point. Here, we take $\mathcal{M}_{(0)}=0$ for the case $m=0$ from the theoretical SBCS ground. The values in Table VI are also shown in Fig. 16 depicting the feature of "Regge trajectories" for the quarkantiquark bound states each with the corresponding equal bare mass $m[1,8,11,12,34]$. It is interesting to note that the Regge trajectory gets slightly modified from just the linear trajectory behavior, developing a bit of curvature in the trajectory for the ground-state and the low-lying excited states. For the small mass, in particular $m=0$, the trajectory looks a little concave shape while for the larger mass the curvature turns somewhat into a convex shape.

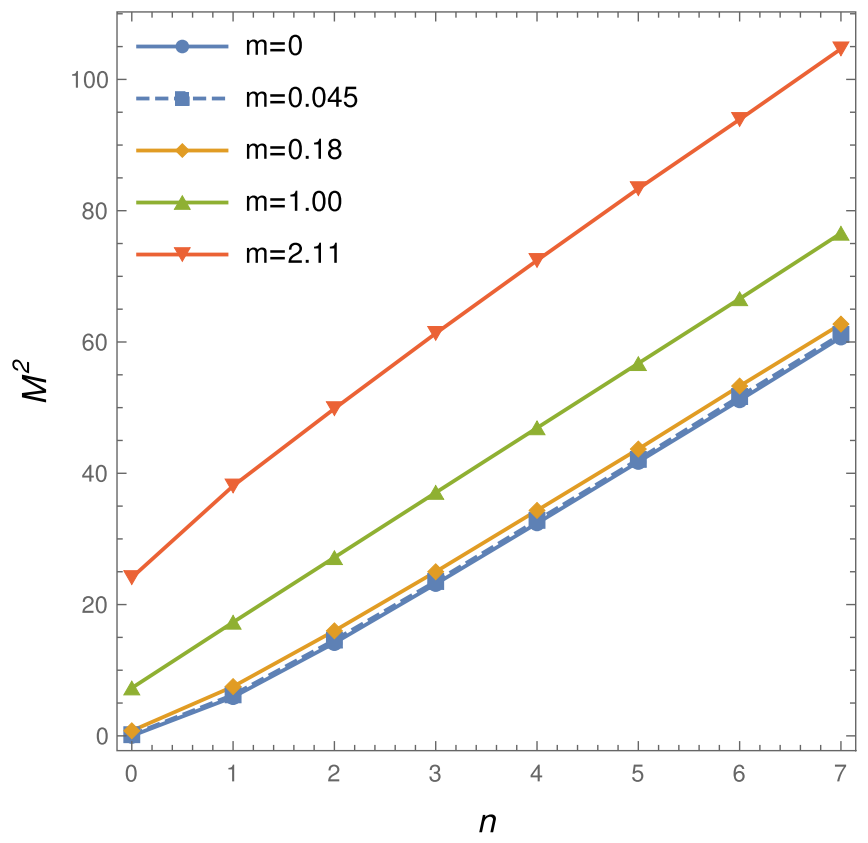

FIG. 16. The feature of "Regge trajectories" for the quarkantiquark bound states each with the corresponding equal bare mass $m$. All quantities are in proper units of $\sqrt{2 \lambda}$. 
This seems to reflect the fact that the GOR works in the chiral limit as shown in Fig. 15 but the chiral symmetry gets broken as the quark mass gets larger. The convex feature of the Regge trajectory for the heavy quarkonia model was shown in Ref. [40].

\section{B. Wave functions}

We present here our numerical solutions of the boundstate wave functions $\hat{\phi}_{ \pm}^{(n)}\left(r_{\hat{\iota}}, x\right)$ interpolating between IFD and LFD for the ground state $n=0$ and the first excited state $n=1$ with $r_{\wedge} \neq 0$ in terms of the interpolating longitudinal momentum fraction variable $x=p_{\wedge} / r_{\wedge}$. The results for $r_{\hat{\iota}}=0$ are presented separately in Appendix $\mathrm{F}$ in terms of the variable $\xi=\tan ^{-1}\left(p_{\hat{\iota}}\right)$ without scaling the interpolating momentum variable $p_{\hat{\imath}}$ with respect to $r_{\hat{\wedge}}$. In the chiral limit, where the GOR relation
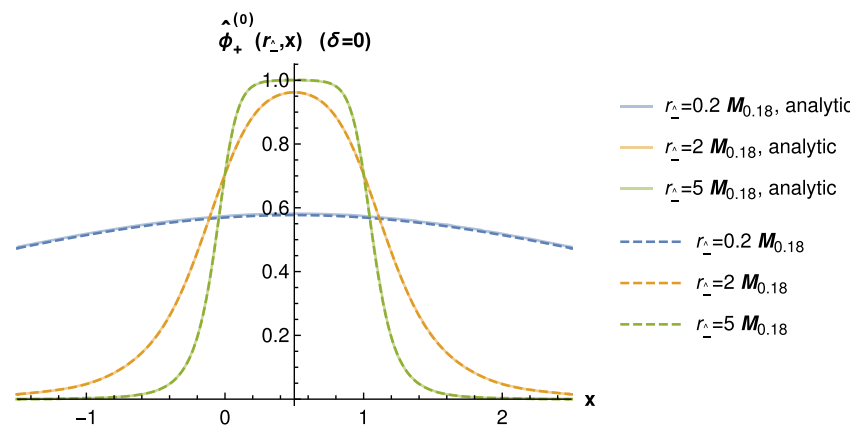

(a)

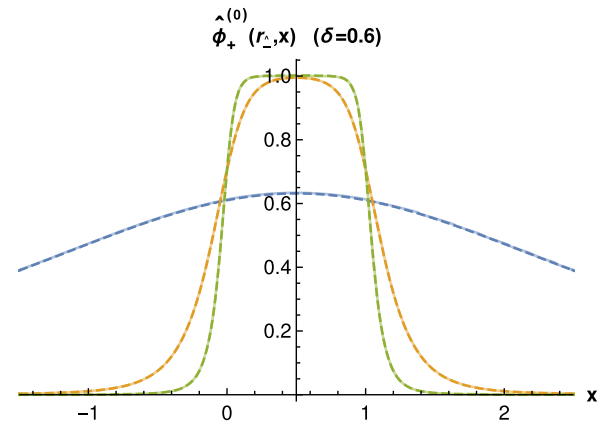

(c)
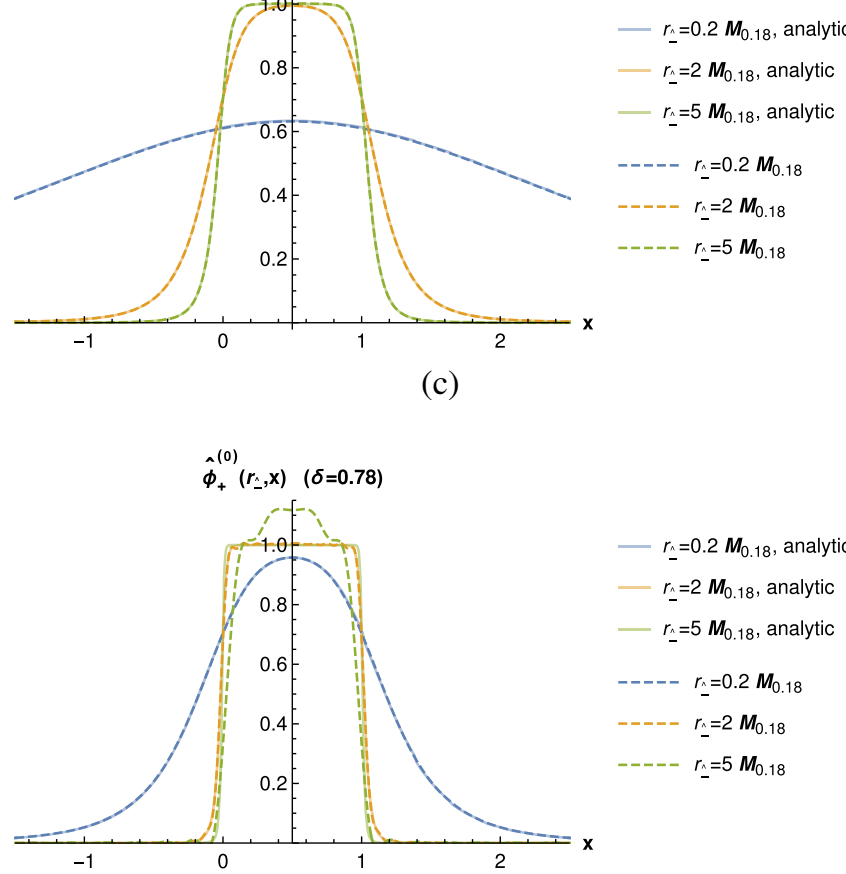

(e)
$\mathcal{M}_{(0)}^{2} \sim m \sqrt{\lambda} \rightarrow 0$ is satisfied, the analytic solution of the pionic ground-state wave function $\phi_{ \pm}$in IFD, i.e., $\hat{\phi}_{ \pm}^{(0)}$ for $\delta=0$, is known [30] in terms of the IFD longitudinal momentum variables $p^{1}$ and $r^{1}$ of the quark and the pionic meson, respectively. Corresponding the IFD longitudinal momentum $p^{1}$ and $r^{1}$ to the interpolating longitudinal momentum $p_{\text {^ }}$ and $r_{\text {^ }}$ and confirming the consistency with the LFD analytic mass gap solution discussed in Sec. II C, we note that the corresponding analytic solution in the interpolating formulation is given by

$$
\begin{aligned}
& \hat{\phi}_{ \pm}^{(0)}\left(r_{\hat{\lrcorner}}, p_{\hat{\lrcorner}}\right)=\frac{1}{2}\left(\cos \frac{\theta\left(r_{\hat{\lrcorner}}-p_{\hat{\lrcorner}}\right)-\theta\left(p_{\hat{\varkappa}}\right)}{2}\right. \\
& \left. \pm \sin \frac{\theta\left(r_{\hat{\iota}}-p_{\hat{\iota}}\right)+\theta\left(p_{\hat{\iota}}\right)}{2}\right) \text {, }
\end{aligned}
$$

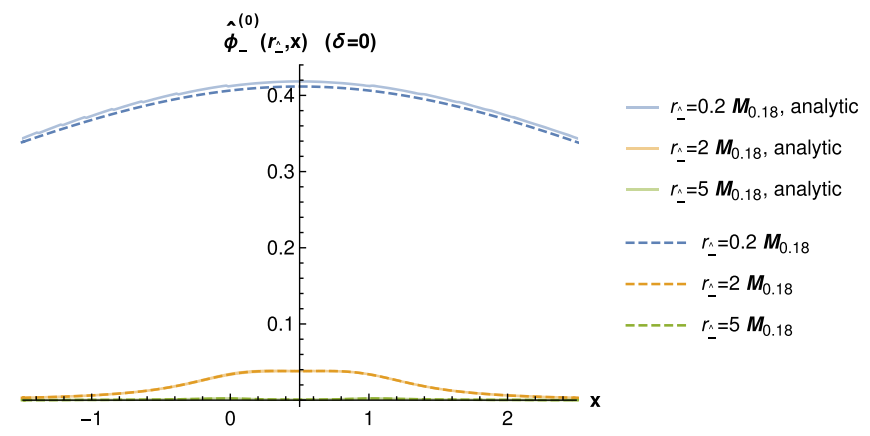

(b)

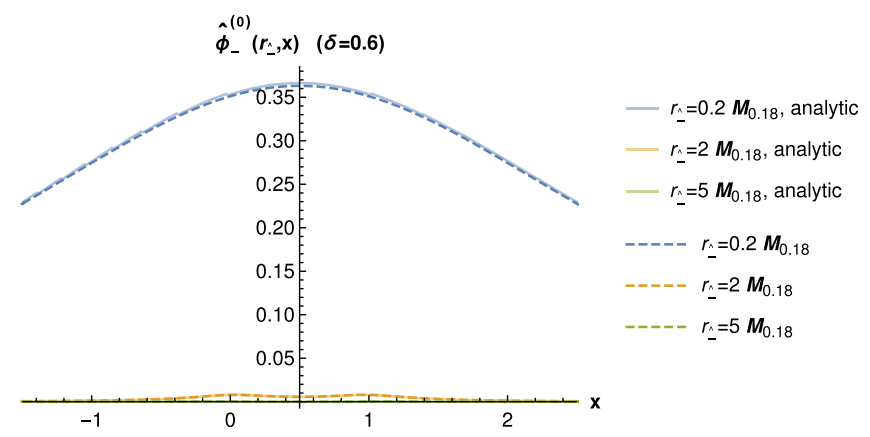

(d)

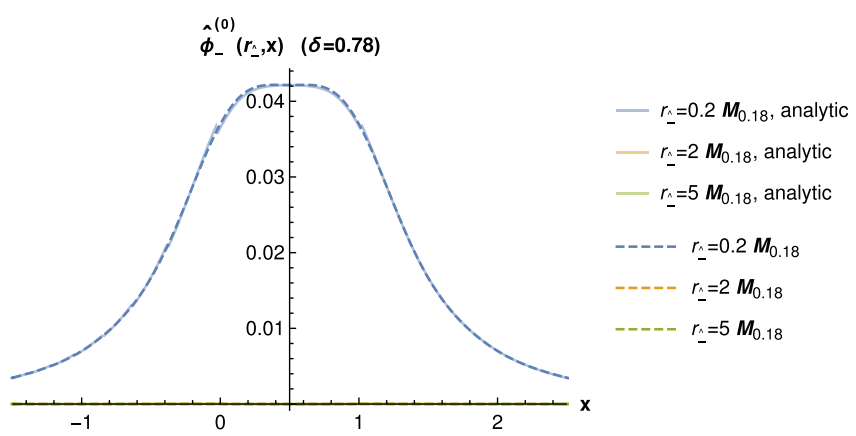

(f)

FIG. 17. Ground state wave functions $\hat{\phi}_{+}^{(0)}\left(r_{\lrcorner}, x\right)$ and $\hat{\phi}_{-}^{(0)}\left(r_{\iota}, x\right)$ for $m=0$. All quantities are in proper units of $\sqrt{2 \lambda}$. 
where the normalization is taken to satisfy Eq. (124) [12]. In Fig. 17, our numerical results of the plus and minus components of the bound-state wave function for the ground state, i.e., $\hat{\phi}_{ \pm}^{(0)}\left(r_{\hat{\iota}}, x\right)$, are shown for the bare quark mass value $m=0$ in comparison with the interpolating analytic solution given by Eq. (128). The results of $\delta=0$, 0.6 and 0.78 are shown in the top, middle, and bottom panels, respectively. In each panel, the results of $r_{\hat{\wedge}}=$ $0.2 \mathbf{M}_{0.18}, 2 \mathbf{M}_{0.18}$ and $5 \mathbf{M}_{0.18}$ with the scale of the groundstate meson mass $\mathbf{M}_{0.18}=0.88$, i.e., $r_{\hat{\wedge}}=0.176,1.76$ and 4.4 (all in units of $\sqrt{2 \lambda}$ ), are depicted by the solid lines for the analytic results and the dashed lines for the numerical results in blue, yellow, and green, respectively. Our numerical results coincide with the analytic results given by Eq. (128) as shown in Fig. 17 except for some wiggle and bulge in the numerical result of $\delta=0.78$ and $r_{\hat{\imath}}=$ $5 \mathbf{M}_{0.18}$ in Fig. 17(e) due to the numerical sensitivity near the LFD $(\mathbb{C} \rightarrow 0)$ and large longitudinal momentum $r_{\wedge}$. Our results in Fig. 17 appear to confirm the validity of our numerical results as well as the analytic results. As the longitudinal momentum $r_{\hat{\imath}}$, i.e., $r^{1}$ for $\delta=0$, gets large, the numerical results of $\hat{\phi}_{+}^{(0)}\left(r_{\hat{\iota}}, x\right)$ approach to $\phi(x)=1$ for $x \in[0,1]$, which is the solution of the 't Hooft equation given by Eq. (118) in LFD [1,34], while $\hat{\phi}_{-}^{(0)}\left(r_{\wedge}, x\right)$ results tend to diminish although for very small momentum, e.g., $r^{1}=0.2 \mathbf{M}_{0.18}$, it is still of comparable order of magnitude to $\hat{\phi}_{+}^{(0)}\left(r_{\lrcorner}, x\right)$ as noted also in Ref. [12]. Reference [22] also noted that for light mesons the large-momentum IFD

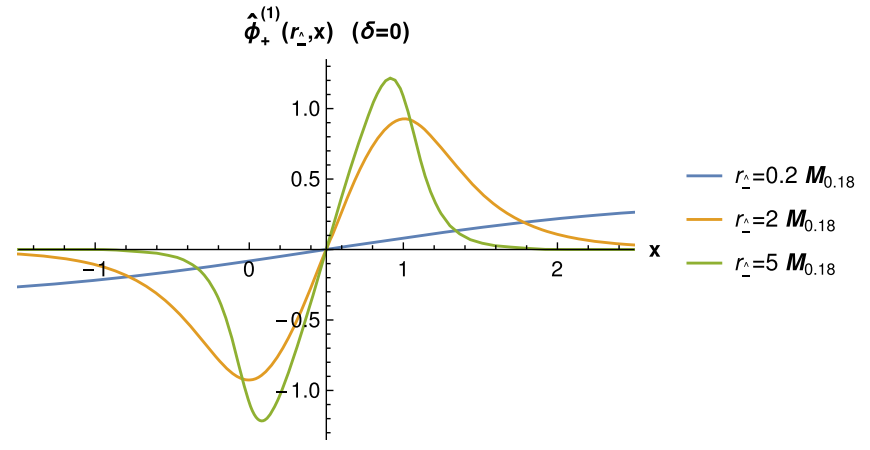

(a)

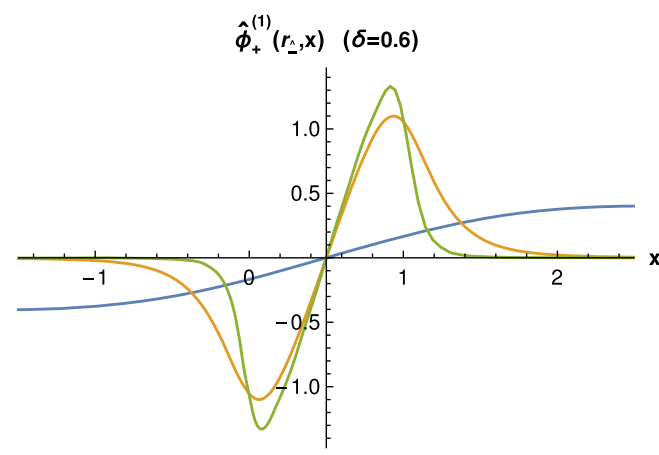

(c)

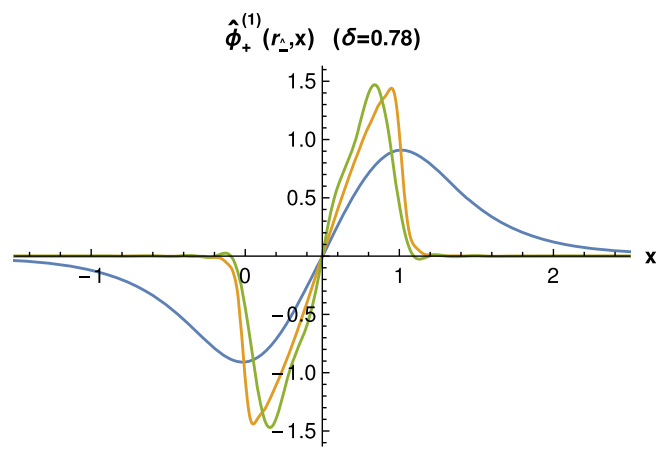

(e)

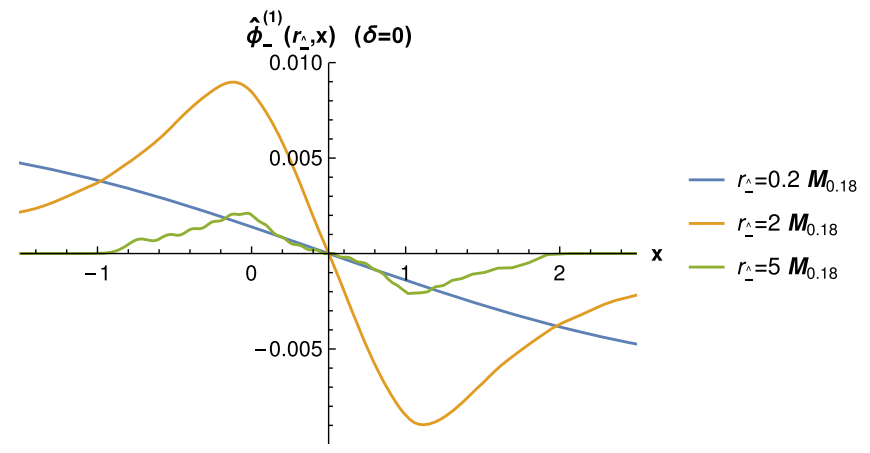

(b)

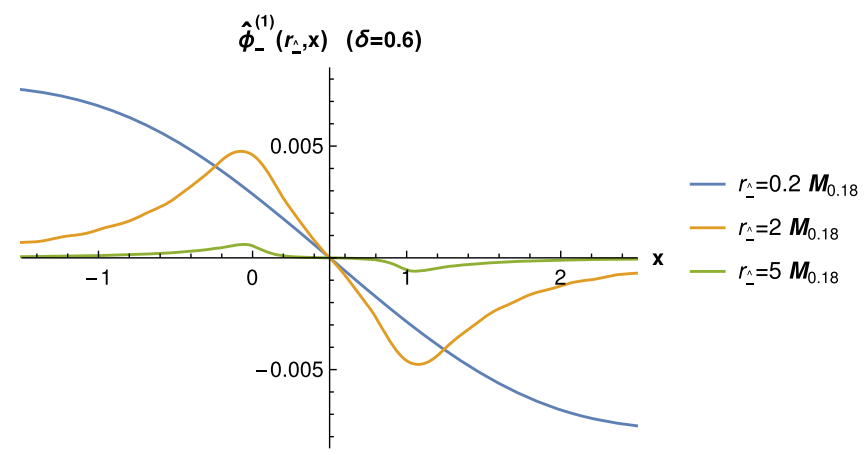

(d)

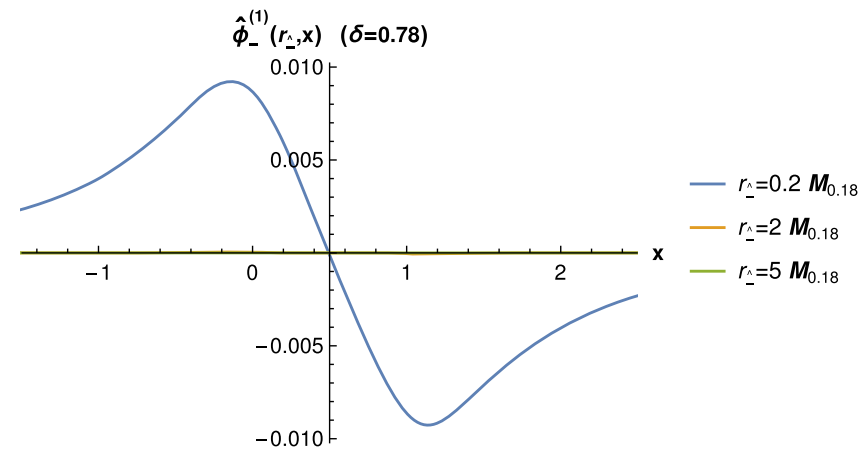

(f)

FIG. 18. First excited state wave functions $\hat{\phi}_{+}^{(1)}\left(r_{\lrcorner}, x\right)$ and $\hat{\phi}_{-}^{(1)}\left(r_{\hat{\iota}}, x\right)$ for $m=0$. All quantities are in proper units of $\sqrt{2 \lambda}$. 
numerical results approach the exact light-front solution very slowly. While the LFD solution for $m=0$ exhibits an infinite slope at the endpoints $x=0,1$, such feature is not achieved in the IFD large momentum method [12,22]. As $\delta$ gets closer to $\pi / 4$, however, the resemblance to the LFD solutions is attained even in the smaller longitudinal momentum (e.g., $r_{\hat{\imath}}=2 \mathbf{M}_{0.18}$ ) and thus the large $r_{\hat{\imath}}$ (e.g., $r_{\hat{\imath}}=5 \mathbf{M}_{0.18}$ ) does not need to be taken for the confirmation of the LFD solutions. The similar behavior of resemblance to the LFD results depending on the values of $\delta$ and $r_{\lrcorner}$is also found in the first excited states $\hat{\phi}_{+}^{(1)}\left(r_{\hat{\iota}}, x\right)$ and $\hat{\phi}_{-}^{(1)}\left(r_{-}, x\right)$ shown in Fig. 18. As dictated by the charge conjugation symmetry, under the exchange of $x \leftrightarrow 1-x$, $\hat{\phi}_{+}^{(1)}\left(r_{-}, x\right)$ and $\hat{\phi}_{-}^{(1)}\left(r_{\lrcorner}, x\right)$ are antisymmetric while $\hat{\phi}_{+}^{(0)}\left(r_{\lrcorner}, x\right)$ and $\hat{\phi}_{-}^{(0)}\left(r_{\lrcorner}, x\right)$ are symmetric. We note also that our results for $\delta=0$ shown in the top panels of Figs. 17-18, i.e., the plots of Figs. 17(a), 17(b), 18(a), 18(b) appear to be consistent with the results in Ref. [12] although only a qualitative comparison can be made as different momentum values are taken for the moving frames in Ref. [12] compared to what we present here.

In contrast to the case of $m=0$, there are no known analytic solutions of the bound-state wave functions for $m \neq 0$. While we present the numerical results of $\hat{\phi}_{+}^{(0)}\left(r_{\lrcorner}, x\right)$ and $\hat{\phi}_{+}^{(1)}\left(r_{\hat{\imath}}, x\right)$ for the cases of $m=0.045$, 1.0 and 2.11 in Appendix D, we take here $m=0.18$ to

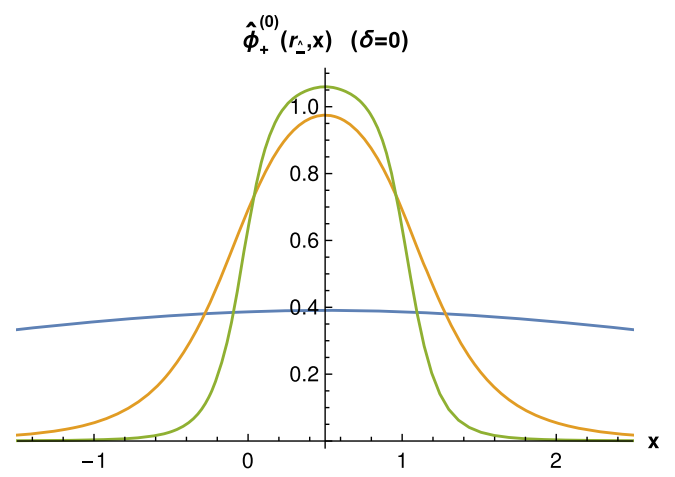

(a)

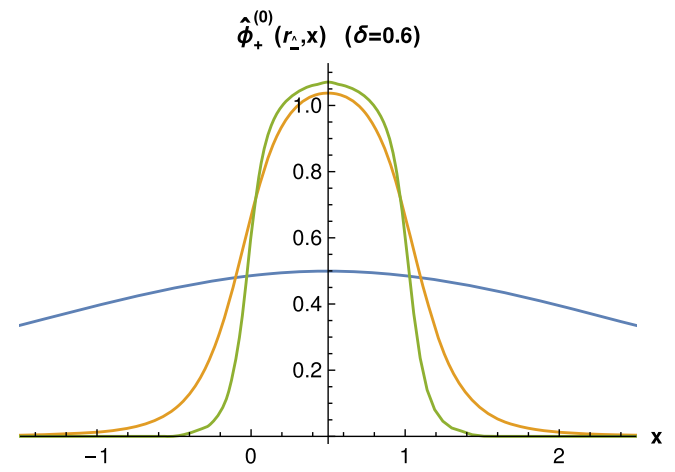

(c)

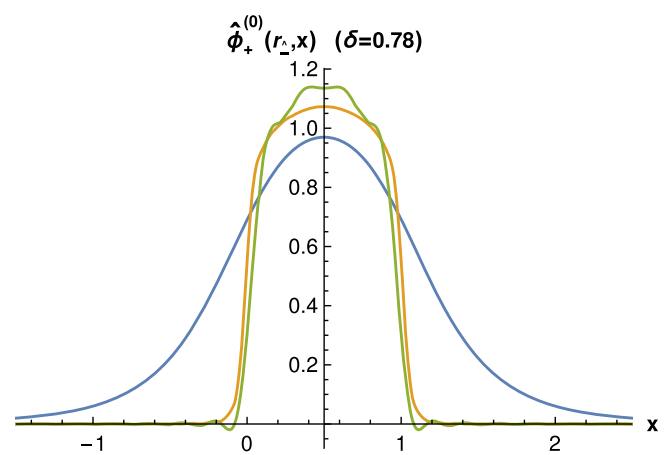

(e)
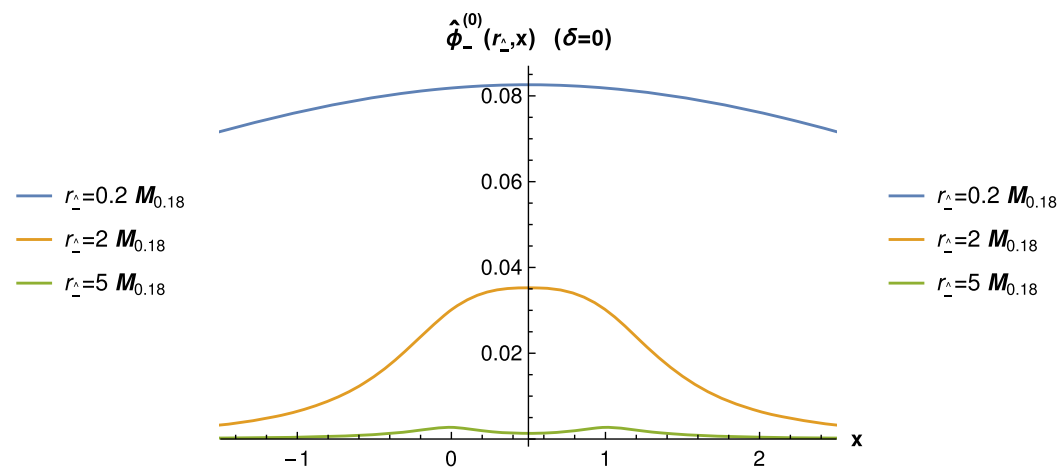

(b)
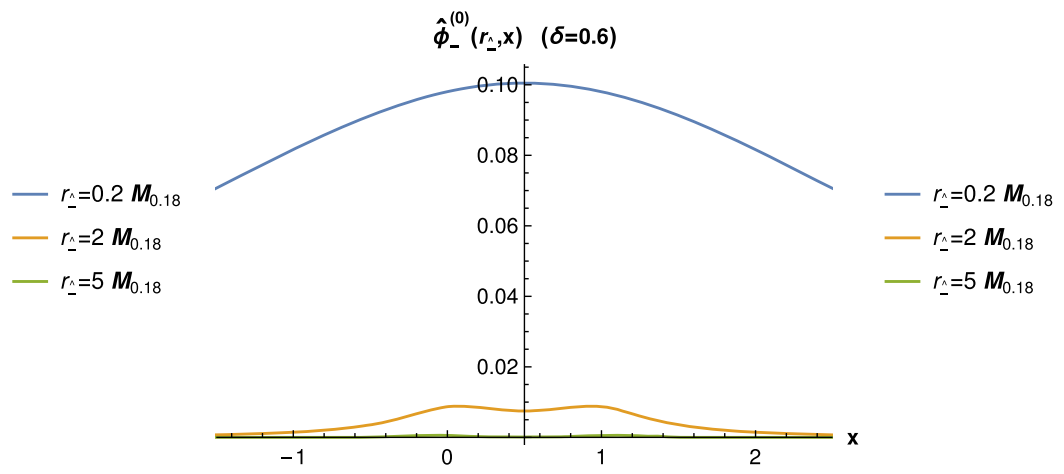

(d)
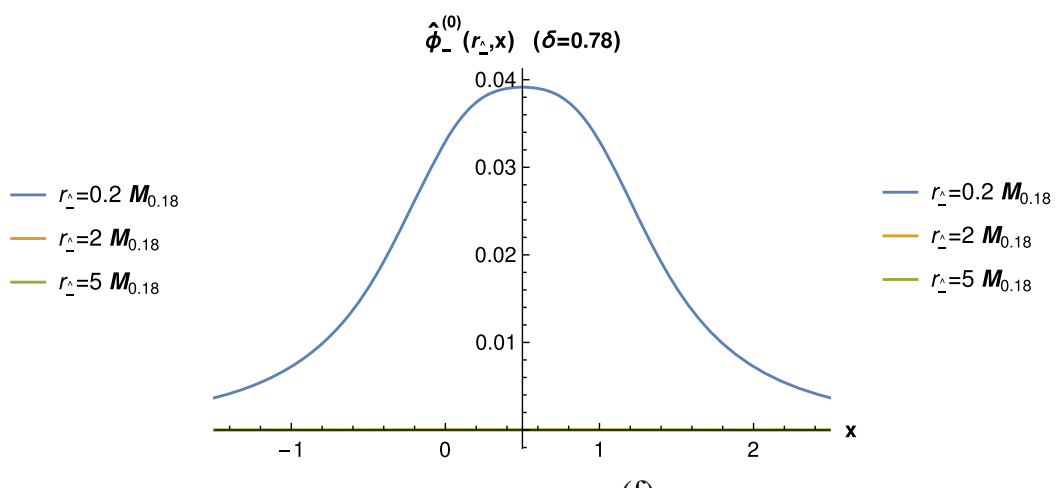

(f)

FIG. 19. Ground state wave functions $\hat{\phi}_{+}^{(0)}\left(r_{\hat{\iota}}, x\right)$ and $\hat{\phi}_{-}^{(0)}\left(r_{\hat{\iota}}, x\right)$ for $m=0.18$. All quantities are in proper units of $\sqrt{2 \lambda}$. 
correspond with the spectroscopy discussion in the last subsection (Sec. VI A) and show its numerical results of $\hat{\phi}_{ \pm}^{(n)}\left(r_{\star}, x\right)$ for the ground state $n=0$ and the first excited state $n=1$. In Fig. 19, the numerical results of $\hat{\phi}_{+}^{(0)}\left(r_{\lrcorner}, x\right)$ and $\hat{\phi}_{-}^{(0)}\left(r_{\lrcorner}, x\right)$ for $\delta=0,0.6$, and 0.78 are shown in the top, middle, and bottom panels, respectively. Similarly, in Fig. 20, the numerical results of $\hat{\phi}_{+}^{(1)}\left(r_{\hat{\iota}}, x\right)$ and $\hat{\phi}_{-}^{(1)}\left(r_{\hat{\iota}}, x\right)$ for $\delta=0,0.6$, and 0.78 are shown in the top, middle and bottom panels, respectively. In each panel, the results of $r_{\hat{\imath}}=0.2 \mathbf{M}_{0.18}, 2 \mathbf{M}_{0.18}$ and $5 \mathbf{M}_{0.18}$ with the scale of $\mathbf{M}_{0.18}=0.88$, i.e., $r_{\hat{\imath}}=0.176,1.76$ and 4.4 (all in units of $\sqrt{2 \lambda}$ ), are depicted by the solid lines in blue, yellow, and green, respectively. As noted for the case of $m=0$, the large-momentum IFD $(\delta=0)$ numerical results approach

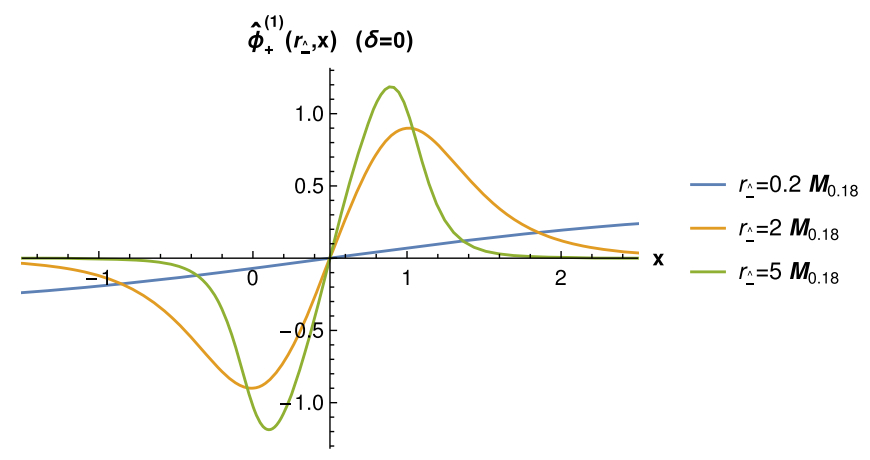

(a)

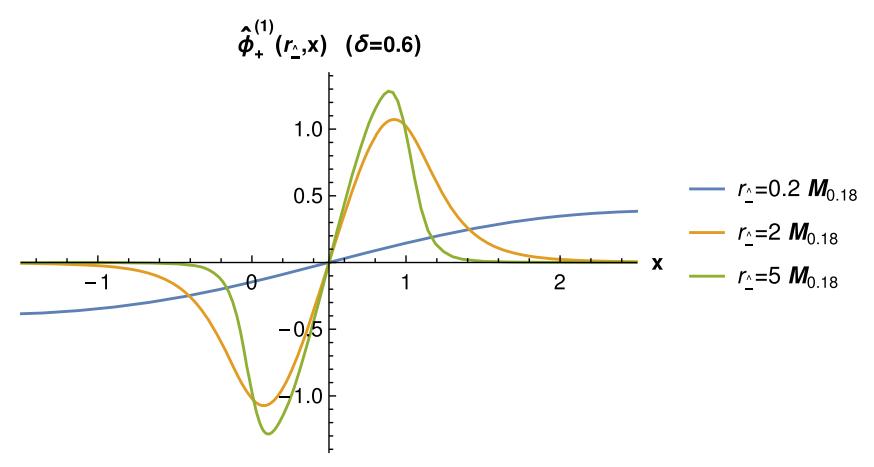

(c)

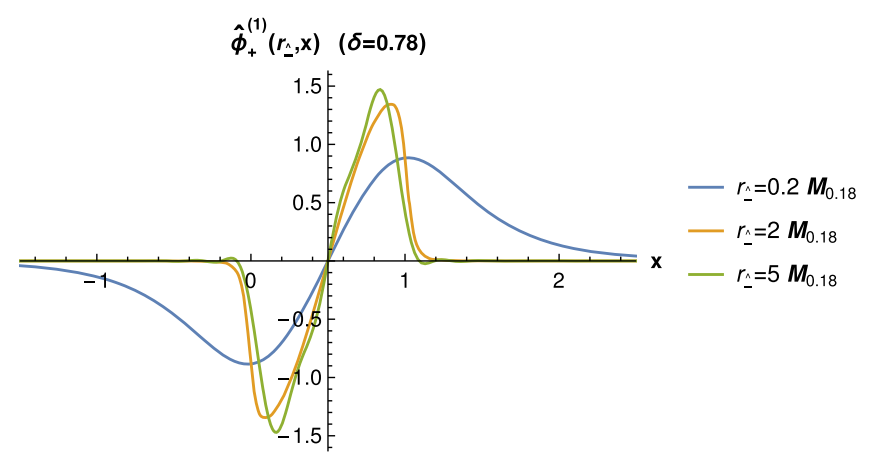

(e) the LFD results very slowly also for the case of $m=0.18$. While the LFD results $\left(\hat{\phi}_{+}^{(n)}\left(r_{\hat{\iota}}, x\right)=\phi^{(n)}(x)\right)$ should be constrained in the $x$-region $[0,1]$, the IFD results in the top left panel of Fig. 19, i.e., Fig. 19a, exhibit rather long tails outside the $[0,1]$ region even for the pretty large longitudinal momentum $r^{1}=5 \mathbf{M}_{0.18}=4.4$. For $\delta=0.78$, however, i.e., very close to the LFD $(\pi / 4 \approx 0.785398)$, shown in Fig. 19e, the wave functions for the relatively larger momenta $r_{\text {乞 }}=2 \mathbf{M}_{0.18}=1.76$ and $5 \mathbf{M}_{0.18}=4.4$ almost coincide with each other while closely fitting in the region $[0,1]$ although the result with very small longitudinal momentum $\left(r_{\hat{\imath}}=0.2 \mathbf{M}_{0.18}=0.176\right)$ has a long tail outside the $[0,1]$ region similar to the IFD $(\delta=0)$ result. While we notice some wiggle and bulge in $\hat{\phi}_{+}^{(0)}\left(r_{\hat{\varkappa}}, x\right)(\delta=$ 0.78) for $r_{\hat{\iota}}=5 \mathbf{M}_{0.18}$, we did not pursue any further

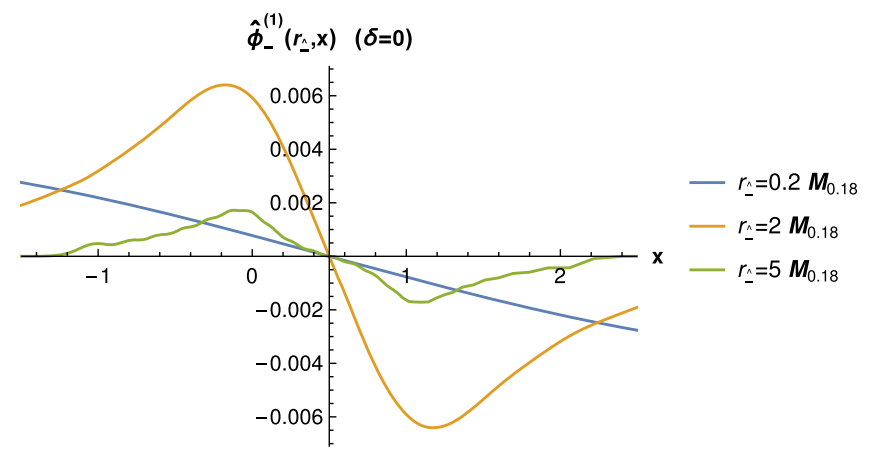

(b)

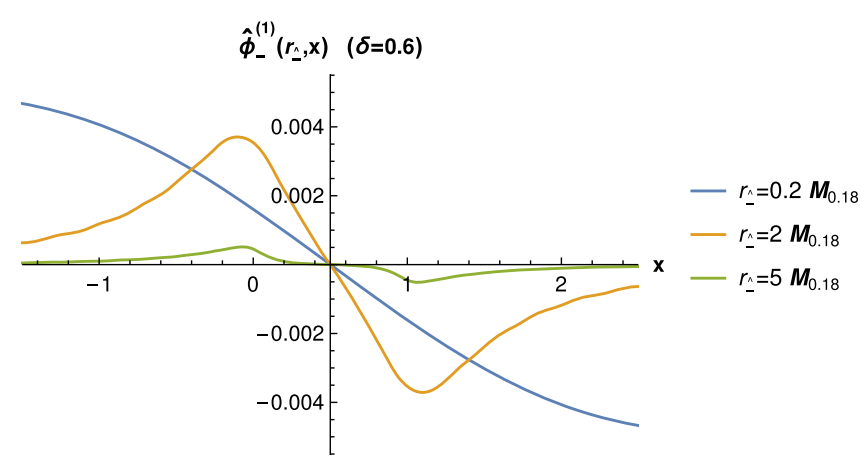

(d)

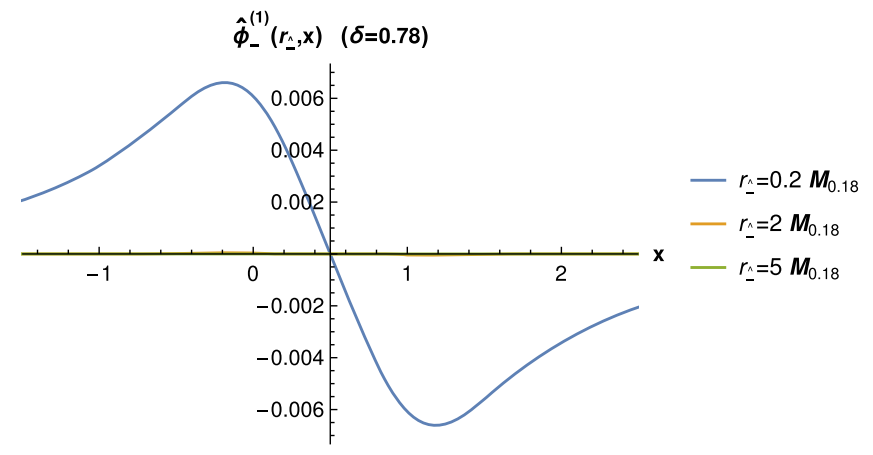

(f)

FIG. 20. First excited state wave functions $\hat{\phi}_{+}^{(1)}\left(r_{\underline{\Delta}}, x\right)$ and $\hat{\phi}_{-}^{(1)}\left(r_{\underline{\Delta}}, x\right)$ for $m=0.18$. All quantities are in proper units of $\sqrt{2 \lambda}$. 
numerical accuracy as we understand that it is due to the computational sensitivity arising in the interpolation region where $\mathbb{C}$ gets close to 0 in particular as $r_{\text {A }}$ gets very large. Since the LFD results of $\hat{\phi}_{-}^{(n)}\left(r_{\hat{-}}, x\right)$ must vanish as discussed in the derivation of the 't Hooft's bound-state equation given by Eq. (118), it is manifest in Fig. 19(b) that the large-momentum IFD $(\delta=0)$ results approach to the $\operatorname{LFD}(\delta=\pi / 4)$ result again very slow for $\hat{\phi}_{-}^{(0)}\left(r_{\wedge}=r^{1}, x\right)$ while the $\delta=0.78$ results in Fig. 19f are rather immediately close to the LFD result. Figure 20 shows the first excited state wave functions $\hat{\phi}_{+}^{(1)}\left(r_{\hat{\iota}}, x\right)$ and $\hat{\phi}_{-}^{(1)}\left(r_{\hat{\iota}}, x\right)$ for the input bare quark mass value $m=0.18$. The results for $m=0.18$ in Fig. 20 look quite similar to the results for $m=0$ in Fig. 18. They share the same feature of the charge conjugation symmetry, under the exchange of $x \leftrightarrow 1-x$, i.e., $\hat{\phi}_{+}^{(1)}\left(r_{\wedge}, x\right)$ and $\hat{\phi}_{-}^{(1)}\left(r_{\lrcorner}, x\right)$ are antisymmetric while $\hat{\phi}_{+}^{(0)}\left(r_{\lrcorner}, x\right)$ and $\hat{\phi}_{-}^{(0)}\left(r_{\lrcorner}, x\right)$ are symmetric. They also share the similar behavior of resemblance to the LFD results depending on the values of $\delta$ and $r_{\hat{\imath}}$, which we have discussed for the ground state previously.

\section{Quasi-PDFs}

Since we obtained the bound-state wave functions, we now apply them to compute the so-called quasi-PDFs which have been discussed extensively even in the 't Hooft model application [22] due to the possibility of computing directly the longitudinal momentum fraction $x$-dependence of the parton distributions in Euclidean lattice approach using the large momentum effective field theory (LaMET) program [41]. In our interpolating 't Hooft model computation, the "quasi-PDFs" may be defined as the following matrix element for the $n$th state of the meson with the interpolating longitudinal momentum $r_{\hat{\Lambda}}$ :

$$
\begin{aligned}
& \tilde{q}_{(n)}\left(r_{\hat{\lrcorner}}, x\right)=\int_{-\infty}^{+\infty} \frac{d x^{\hat{\wedge}}}{4 \pi} \mathrm{e}^{i x^{\wedge} r_{\varkappa}}\left\langle r_{(n)}^{\hat{+}}, r_{\hat{\sim}}\right| \bar{\psi}\left(x^{\hat{\varkappa}}\right) \\
& \times \gamma_{\hat{\lrcorner}} \mathcal{W}\left[x^{\hat{\alpha}}, 0\right] \psi(0)\left|r_{(n)}^{\hat{+}}, r_{\hat{\lrcorner}}\right\rangle_{C},
\end{aligned}
$$

where $r_{(n)}^{\hat{+}}=\sqrt{r_{\hat{\varkappa}}^{2}+\mathbb{C} \mathcal{M}_{(n)}^{2}}$ as obtained from Eq. (7), and the range of the longitudinal momentum fraction $x=p_{\wedge} / r_{\text {A }}$ is unconstrained, $-\infty<x<+\infty$, for $0 \leq \delta<\pi / 4$, while bounded, $0 \leq x \leq 1$, for $\delta=\pi / 4$. One should note that this definition of the "quasi-PDFs" is not unique, e.g., taking $\gamma^{\hat{f}}$ instead of $\gamma_{\wedge}$ in front of the interpolating gauge link $\mathcal{W}\left[x^{\wedge}, 0\right]$ in Eq. (129), but still uniquely approach the PDF defined in the LFD as $\delta \rightarrow \pi / 4$, whichever definition is taken. While one may consider the so-called universality class [42] of the interpolating "quasi-PDFs," we note that the definition given by Eq. (129) coincides with the canonical definition of the quasi-PDFs in IFD $(\delta=0)$ [22]. While it has been discussed which definition approaches the PDFs in LFD faster for the perspectives of the LaMET program $[22,41]$, we will take the definition given by Eq. (129) in this work and discuss our numerical results corresponding to this definition. The interpolating gauge link

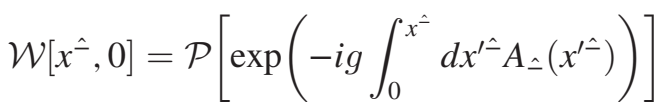

inserted in Eq. (129) assures the gauge invariance of the interpolating "quasi-PDFs". The subscript $C$ in Eq. (129) indicates the removal of the disconnected contribution discussed [43] for the forward matrix element computation:

$$
\begin{aligned}
& \left\langle r_{(n)}^{\hat{+}}, r_{\hat{\wedge}} \mid r_{(n)}^{\hat{+}}, r_{\hat{\Lambda}}\right\rangle
\end{aligned}
$$

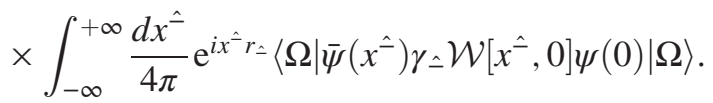

As we adopted the axial gauge in the interpolation form, i.e., $A_{-}^{a}=0$, the gauge link becomes an identity and the quantization procedure illustrated in Sec. II A yields

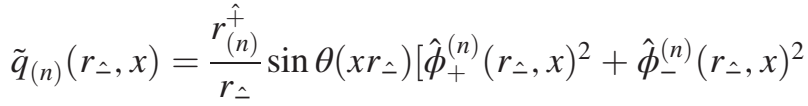

$$
\begin{aligned}
& \left.+\hat{\phi}_{+}^{(n)}\left(r_{\hat{\lrcorner}},-x\right)^{2}+\hat{\phi}_{-}^{(n)}\left(r_{\hat{\iota}},-x\right)^{2}\right] .
\end{aligned}
$$

For $\delta=0$, i.e., IFD, Eq. (132) coincides with Eq. (74) of Ref. [22].

Based on this formula, we compute the interpolating "quasi-PDFs" for the cases of $\delta=0,0.6$, and 0.78. In Figs. 21 and 22, the interpolating "quasi-PDFs" of the ground state $(n=0)$ and the first excited state $(n=1)$ are shown for the case of $\delta=0$, respectively. The two panels in each of these figures exhibit our numerical results for the bare quark mass $m=0$ and $m=0.18$, respectively. Numerical results for other mass cases $(m=0.045,1.0$ and 2.11) are summarized in Appendix E. In each panel, the results for the meson longitudinal momentum $r_{\hat{\wedge}}=r^{1}=$ $0.2 \mathbf{M}_{0.18}, 2 \mathbf{M}_{0.18}, 5 \mathbf{M}_{0.18}$ are depicted by blue, yellow and green solid lines, respectively. The general behaviors of our numerical results with respect to the variation of $r^{1}$ values from small $\left(0.2 \mathbf{M}_{0.18}\right)$ to large $\left(5 \mathbf{M}_{0.18}\right)$ agree with the results presented in Ref. [22], although different longitudinal momentum values were taken between ours and Ref. [22]. As $r^{1}$ gets larger, the numerical results of the quasi-PDFs resemble the PDFs in LFD more closely fitting in $x \in[0,1]$ and $[-1,0]$. It is our interest to take a look at the rate of achieving the resemblance to the PDFs in LFD as $\delta$ gets away from the IFD $(\delta=0)$ and $r_{\imath}$ gets larger. The numerical results of the ground state $(n=0)$ and the first excited state $(n=1)$ are shown for the case of $\delta=0.6$ in Figs. 23 and 24 and for the case of $\delta=0.78$ in Figs. 25 and 26, respectively. As noticed previously in Figs. 17 and 19, the wiggle and bulge in $\hat{\phi}_{+}^{(0)}\left(r_{\hat{\imath}}, x\right)(\delta=0.78)$ for $r_{\hat{\iota}}=$ $5 \mathbf{M}_{0.18}$ is due to the computational sensitivity arising in the 


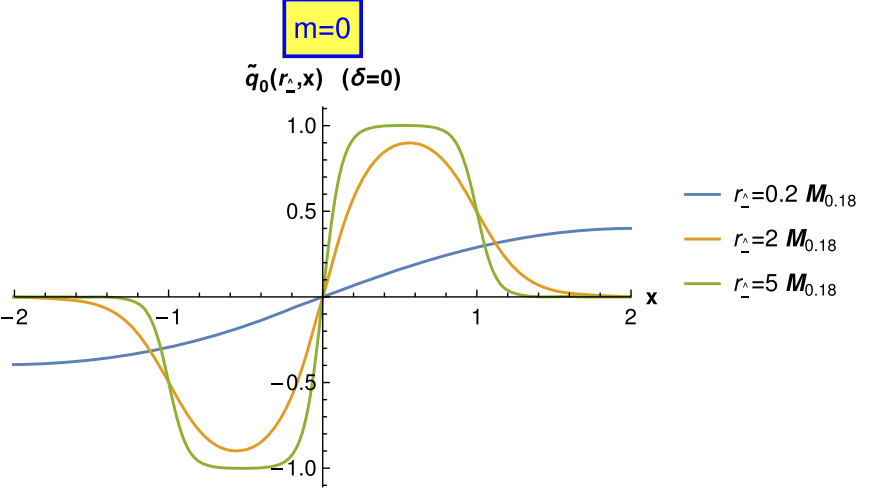

(a)

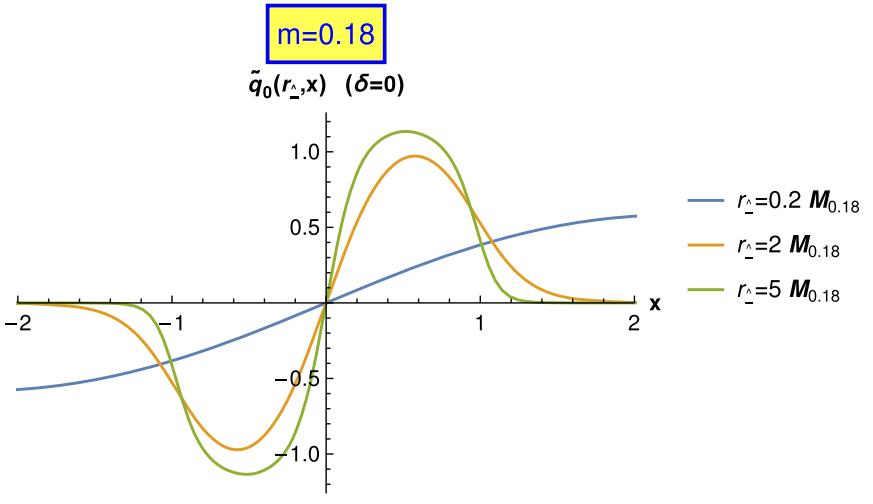

(b)

FIG. 21. Quasi-PDFs in IFD $(\delta=0)$ for the ground state $(n=0)$ wave functions of (a) $m=0$, (b) $m=0.18$. All quantities are in proper units of $\sqrt{2 \lambda}$.

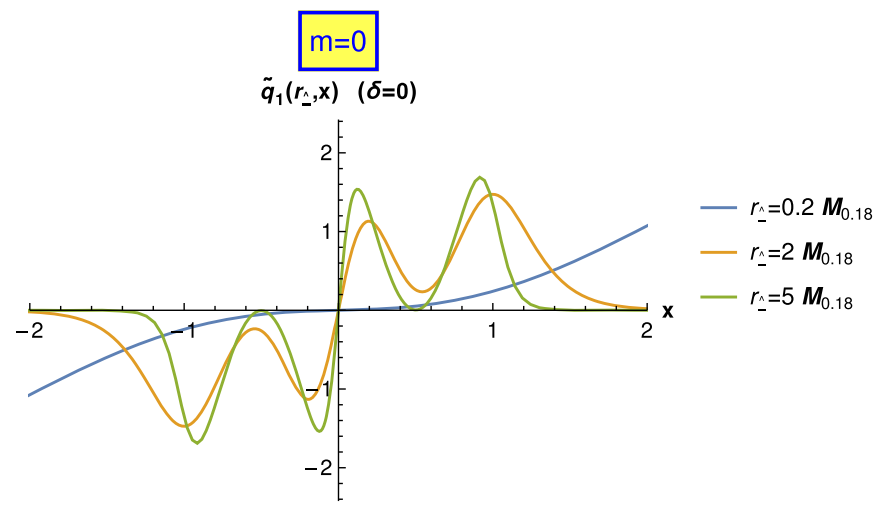

(a)

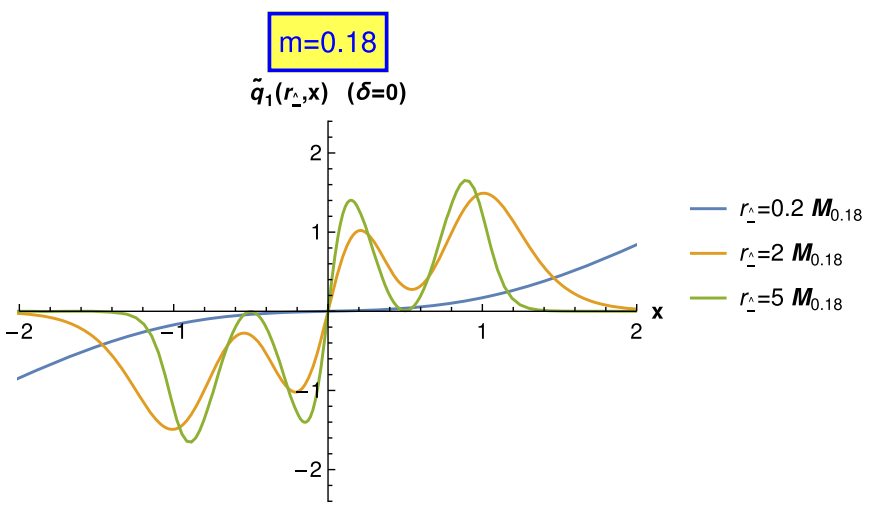

(b)

FIG. 22. Quasi-PDFs in IFD $(\delta=0)$ for the first excited state $(n=1)$ wave functions of (a) $m=0$, (b) $m=0.18$. All quantities are in proper units of $\sqrt{2 \lambda}$.

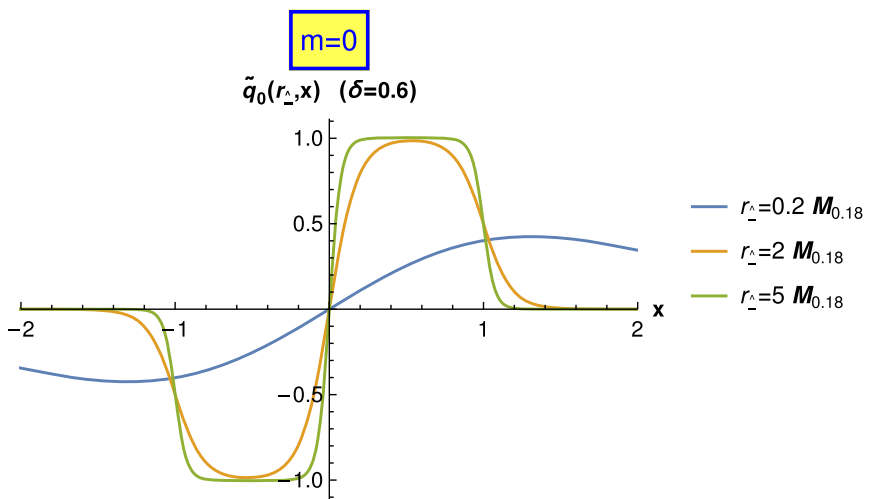

(a)

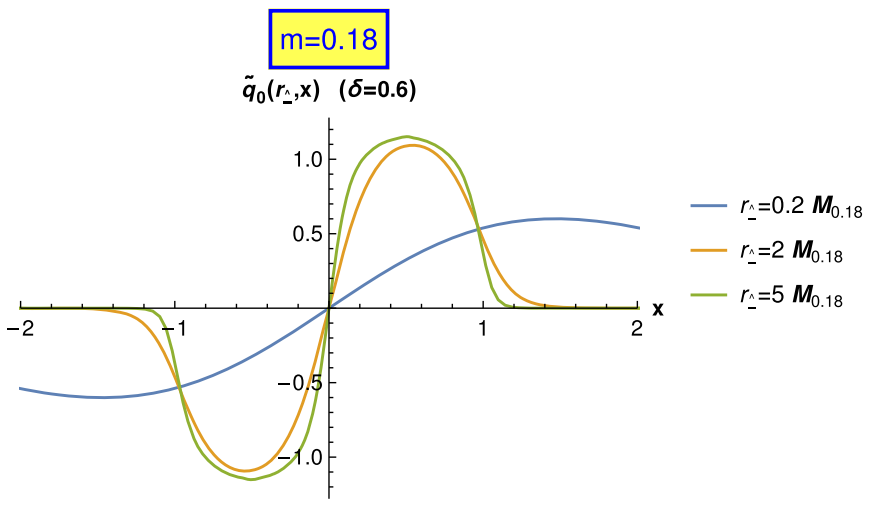

(b)

FIG. 23. $\delta=0.6$ interpolating "quasi-PDFs" for the ground state $(n=0)$ wave functions of (a) $m=0$, (b) $m=0.18$. All quantities are in proper units of $\sqrt{2 \lambda}$. 


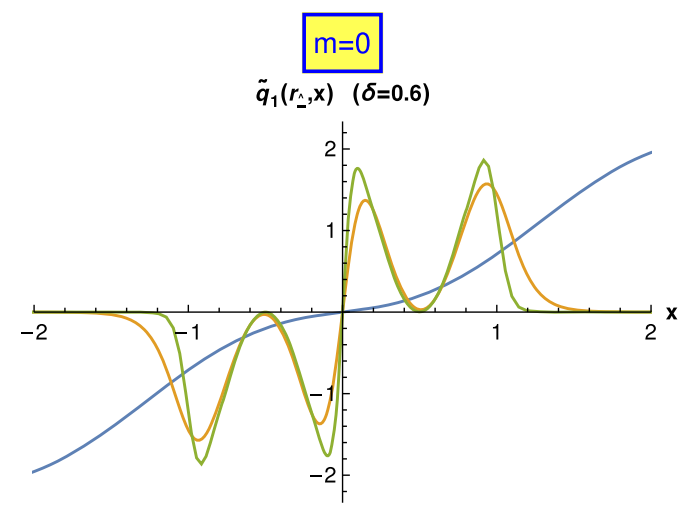

(a)

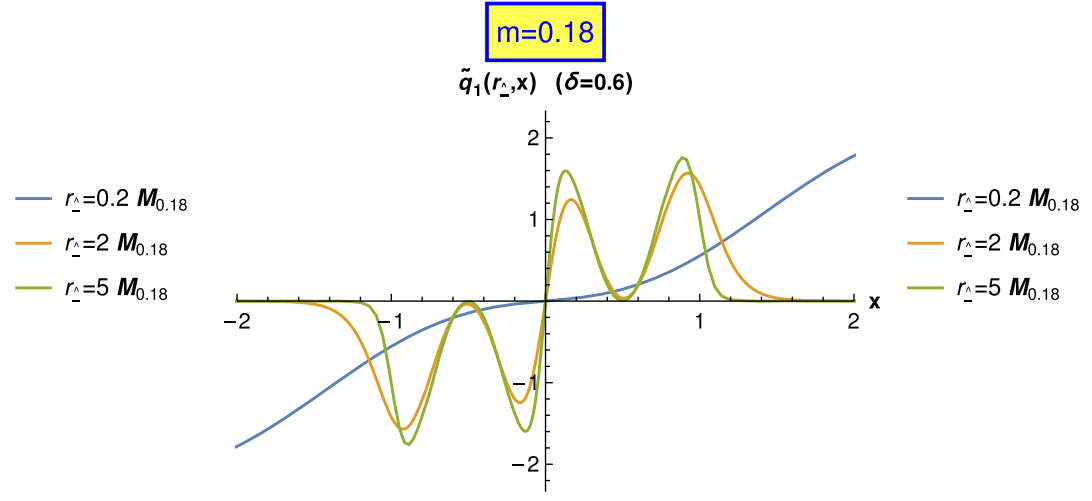

(b)

FIG. 24. $\delta=0.6$ interpolating "quasi-PDFs" for the first excited state $(n=1)$ wave functions of (a) $m=0,(b) m=0.18$. All quantities are in proper units of $\sqrt{2 \lambda}$.

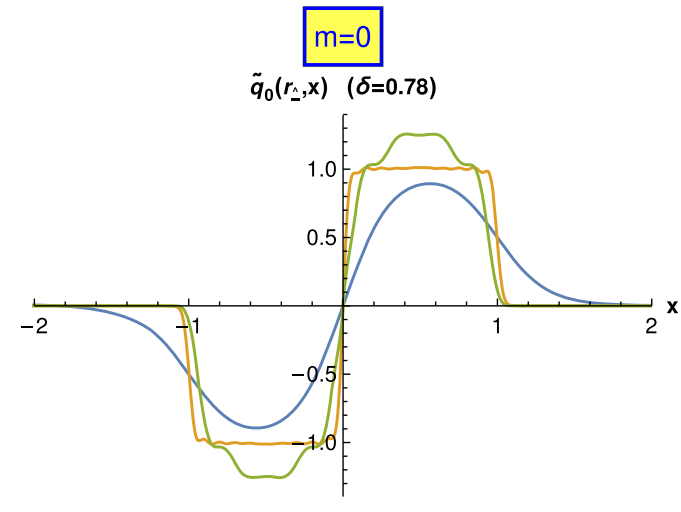

(a)

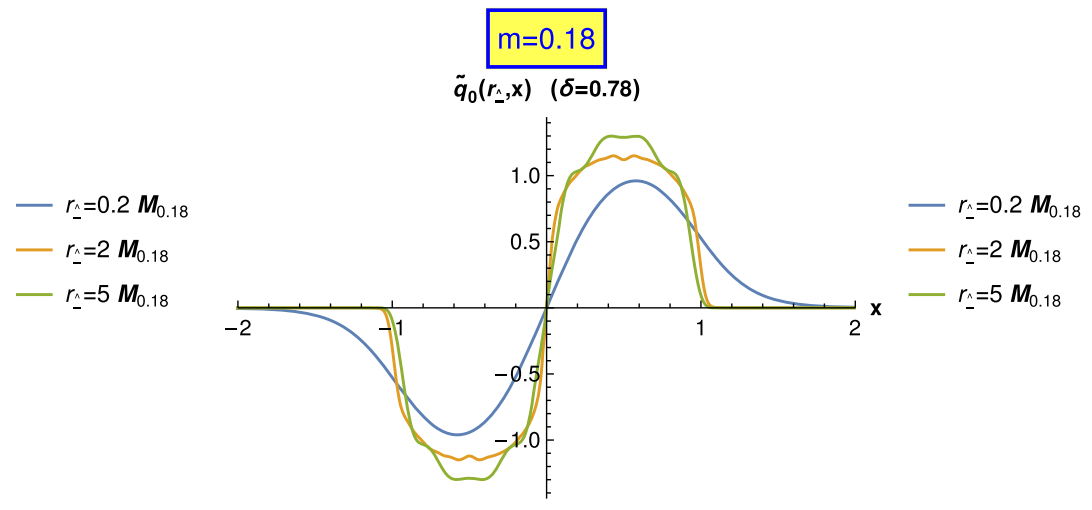

(b)

FIG. 25. $\delta=0.78$ interpolating "quasi-PDFs" for the ground state $(n=0)$ wave functions of (a) $m=0$, (b) $m=0.18$. All quantities are in proper units of $\sqrt{2 \lambda}$.

interpolation region where $\mathbb{C}$ gets close to 0 in particular as $r_{\text {. }}$ gets very large. The corresponding wiggle and bulge in $\tilde{q}_{(0)}\left(r_{\hat{\iota}}, x\right)$ is noticed also in Fig. 25. Besides such numerical sensitivity for the very large value of $r_{\hat{\lrcorner}}$, it is apparent that the $\delta=0.78$ results are rather immediately close to the LFD result.

Interestingly, our numerical results of the interpolating "quasi-PDFs" in the moving frames indicate a possibility to utilize both variation of $\delta$ and $r_{\text {A }}$ to attain the LFD result more effectively. Namely, one may not need to boost the longitudinal momentum $r_{\wedge}$ too large but search for a "sweet spot" by varying both $\delta$ and $r_{-}$together to obtain the "LFDlike" result. In IFD, $\delta=0$ is fixed and thus the boost to the large longitudinal momentum is necessary for a successful approach to the LFD result. However, in the interpolating formulation between the IFD and the LFD, the LFD result can be approached even at rather small $r_{\hat{A}}$. Moreover, the application to the lattice formulation may be also possible with the existing technique of Wick rotation replacing the ordinary instant form time $x^{0}$ by the interpolating time $x^{\hat{+}}$ in the process of taking the "imaginary time" in the lattice as far as $\delta$ remains in the region $0 \leq \delta<\pi / 4$ avoiding the light-like surface $\delta=\pi / 4$. As discussed in the later part of Sec. IV B, one can match the Minkowsky space and the Euclidean space confirming the correspondence given by Eq. (97).

For an illustration of the $\delta$ variation for a given finite $r_{\hat{\lambda}}$, we take $r_{\hat{\imath}}=2 \mathbf{M}_{0.18}$ for the case of $m=0$ and show the "quasi-PDFs" of the ground-state and the first excited-state for the variation of $\delta$ parameter as $\delta=0, \delta=0.6$ and $\delta=$ 0.78 in Fig. 27. It indicates that a pretty slow approach to the LFD result in the large-momentum IFD can be fairly well expedited by taking $\delta$ away from the IFD $(\delta=0)$ and getting closer to the $\operatorname{LFD}(\delta=\pi / 4)$ while the same value of the longitudinal momentum $r_{\hat{\imath}}=2 \mathbf{M}_{0.18}$ is taken. The numerical sensitivity arising near $\delta=\pi / 4$ for the large $r_{\hat{\wedge}}$, e.g., the "wiggle and bulge" mentioned for $r_{\hat{\imath}}=5 \mathbf{M}_{0.18}$ in Figs. 25 and 26 for $\delta=0.78$, is also dodged by taking the smaller value of $r_{\hat{\iota}}$, e.g., $r_{\hat{\iota}}=2 \mathbf{M}_{0.18}$ for this illustration. We think it would be worthwhile to explore this idea of 


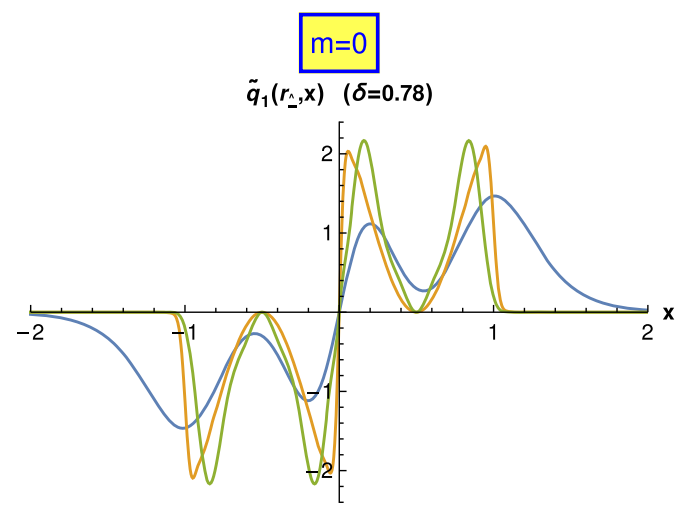

(a)

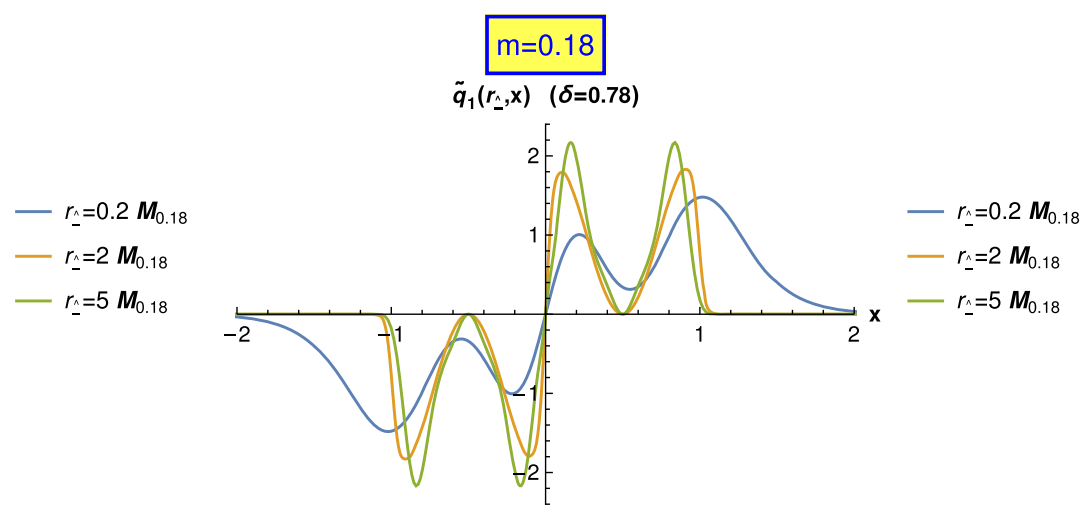

(b)

FIG. 26. $\delta=0.78$ interpolating "quasi-PDFs" for the first excited state $(n=1)$ wave functions of (a) $m=0$, (b) $m=0.18$. All quantities are in proper units of $\sqrt{2 \lambda}$.

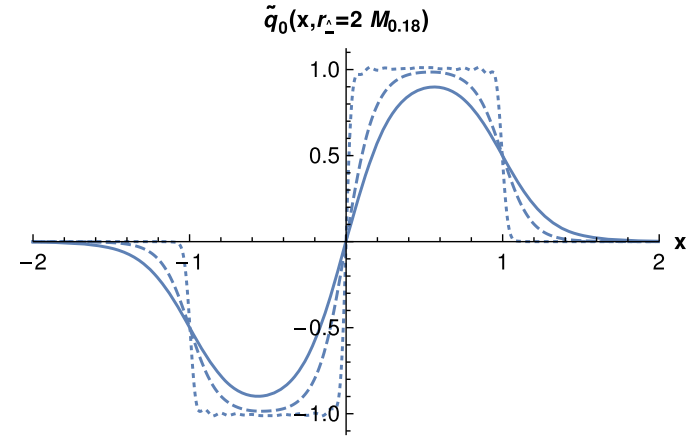

(a)

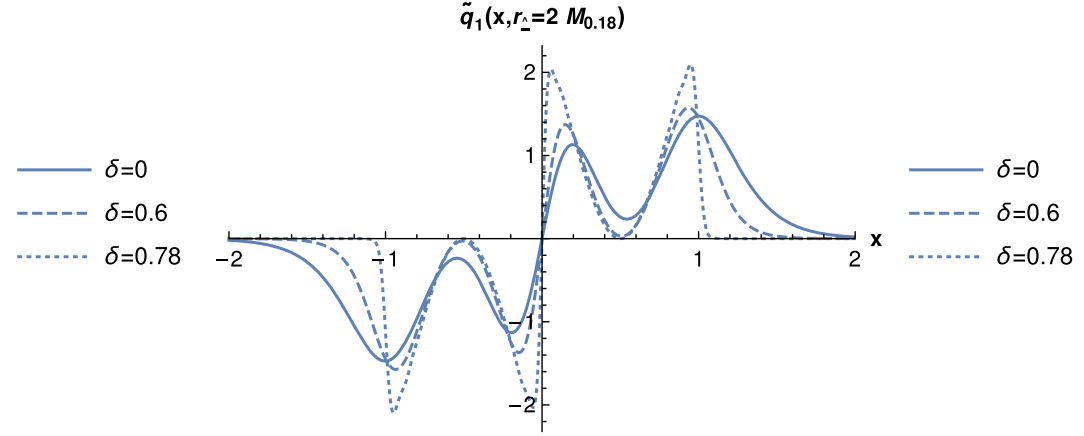

(b)

FIG. 27. Interpolating "quasi-PDFs" at the fixed value of $r_{\hat{\imath}}=2 \mathbf{M}_{0.18}$ for the (a) ground state and (b) first excited state for $m=0$ in three cases of $\delta=0, \delta=0.6$ and $\delta=0.78$. All quantities are in proper units of $\sqrt{2 \lambda}$.

utilizing the interpolating formulation between IFD and LFD for the application to the lattice computation.

For further application of the interpolating bound-state wave functions, one can also consider the interpolating "quasi-distribution amplitude (quasi-DA)" which may be written as

$$
\begin{aligned}
& \tilde{\Phi}_{(n)}\left(r_{\lrcorner}, x\right)=\frac{1}{f^{(n)}} \int_{-\infty}^{+\infty} \frac{d x^{\hat{\imath}}}{2 \pi} \mathrm{e}^{i\left(x-\frac{1}{2}\right) r_{\lrcorner} x^{\wedge}} \\
& \times\left\langle r_{(n)}^{\hat{+}}, r_{\hat{\iota}}\right| \bar{\psi}\left(\frac{x^{\hat{\imath}}}{2}\right) \mathcal{W}\left[\frac{x^{\hat{\imath}}}{2},-\frac{x^{\hat{\imath}}}{2}\right] \\
& \times \gamma_{\wedge} \gamma_{5} \psi\left(-\frac{x^{\hat{A}}}{2}\right)|\Omega\rangle \text {, }
\end{aligned}
$$

where $\mathcal{W}$ is the gauge link introduced in Eq. (130), and $f^{(n)}$ is the decay constant mentioned in Sec. VI A. As mentioned in the definition of the interpolating "quasi-PDFs" given by Eq. (129), the definition of the interpolating "quasi-DAs" is also not unique, e.g., $\gamma^{\hat{+}}$ can be taken instead of $\gamma_{\hat{\epsilon}}$ in front of the interpolating gauge link $\mathcal{W}\left[\frac{x^{\wedge}}{2},-\frac{x^{\hat{2}}}{2}\right]$ in Eq. (133). Whichever definition is taken, they all uniquely approach the DA defined in the LFD as $\delta \rightarrow \pi / 4$, belonging to the same universality class [42] as mentioned for the case of interpolating "quasi-PDFs". Using the definition given by Eq. (133) which coincides with the canonical definition of the quasi-DAs in IFD $(\delta=0)$ [22], we note that the interpolating "quasi-DAs" of the even- $n$ mesonic states can be formulated as

$$
\begin{aligned}
\tilde{\Phi}_{(n)}\left(r_{\hat{\iota}}, x\right)= & \frac{1}{f^{(n)}} \sqrt{\frac{N_{c} r^{\hat{+}}}{\pi r_{\hat{\iota}}}} \sin \frac{\theta\left(x r_{\hat{\iota}}\right)+\theta\left((1-x) r_{\hat{\varkappa}}\right)}{2} \\
& \times\left[\hat{\phi}_{+}^{(n)}\left(r_{\hat{\iota}}, x\right)+\hat{\phi}_{-}^{(n)}\left(r_{\hat{\iota}}, x\right)\right],
\end{aligned}
$$

where $\hat{\phi}_{ \pm}^{(n)}\left(r_{\hat{\imath}}, x\right)$ denote the interpolating mesonic wave functions associated with the $n$th excited mesonic state. The normalization condition of the interpolating "quasi-DAs" given by 


$$
\int_{-\infty}^{+\infty} d x \tilde{\Phi}_{(2 n)}\left(r_{\hat{\imath}}, x\right)=1
$$

is consistent with the explicit form of the decay constant $f^{(n)}$ given by

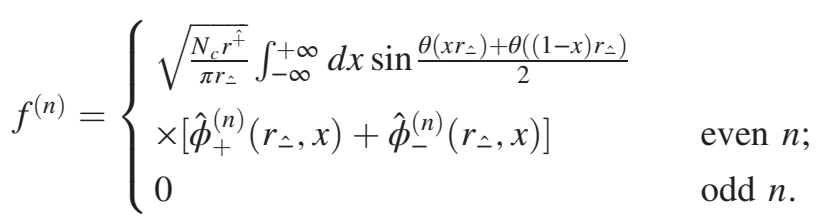

While the corresponding results for the IFD $(\delta=0)$ have been worked out in Refs. [12,22], it is interesting to note that the analytic result for the pion decay constant $f_{\pi}=\sqrt{N_{c} / \pi}$ [30] can be immediately obtained by taking the LFD solution in the chiral limit, i.e., $\hat{\phi}_{+}^{(0)}\left(r_{\hat{\iota}}, x\right)=\phi(x)=1$ for $x \in[0,1]$ and $\hat{\phi}_{-}^{(0)}\left(r_{\hat{\iota}}, x\right)=0$, as well as $\theta\left(x r_{\hat{\iota}}\right)=\theta\left(x r^{+}\right)=\theta\left(p^{+}\right)=$ $\frac{\pi}{2}$ and $\theta\left((1-x) r_{\lrcorner}\right)=\theta\left((1-x) r^{+}\right)=\theta\left(r^{+}-p^{+}\right)=\frac{\pi}{2}$, noting $\frac{r^{+}}{r_{\perp}}=\frac{r^{+}}{r_{-}}=1$ in LFD. As the DAs in LFD are directly involved with the QCD factorization theorem for the hard exclusive reactions involving hadrons, it would be useful to explore the utility of the interpolating "quasi-DAs" further in the future works.

\section{CONCLUSION AND OUTLOOK}

In this work, we interpolated the 't Hooft model (i.e., $\mathrm{QCD}_{2}$ in the large $N_{c}$ limit) with the interpolation angle $\delta$ between IFD $(\delta=0)$ and LFD $(\delta=\pi / 4)$ and analyzed its nontrivial vacuum effects on the quark mass and wave function renormalization as well as the corresponding meson mass and wave function properties taking the meson as the quark-antiquark bound-state. We derived the interpolating mass gap equation between IFD and LFD using not only the algebraic method based on the Bogoliubov transformation between the trivial and nontrivial vacuum as well as the bare and dressed quark but also the diagrammatic method based on the self-consistent embodiment of the quark self-energy. Our mass gap solutions agree not only with the LFD result in Ref. [1] for $\delta=\pi / 4$ but also with the IFD results in Refs. [11,12] for $\delta=0$. The renormalized chiral condensate was computed and the agreement of the result in the chiral limit was verified with the exact result in Ref. [4]. Its invariance regardless of the $\delta$ values between IFD and LFD was also confirmed. Taking into account the wave function renormalization factor $F\left(p_{\iota}\right)$ as well as the mass function $M\left(p_{\iota}\right)$ and expressing the dressed quark propagator $S(p)$ in terms of $F\left(p_{\hat{\iota}}\right)$ and $M\left(p_{\hat{\iota}}\right)$ as given by Eq. (88), we resolved the issue of $E\left(p_{\hat{\varkappa}}\right)$ not being always positive for $m \lesssim 0.56$ discussed in Ref. [10]. Extending the interpolating energymomentum dispersion relation of the on-mass-shell particle given by Eq. (7) to the case of the dressed quark with the rescaled variable given by Eq. (96), we obtained the interpolation angle independent energy function $\tilde{E}\left(p_{\hat{A}}^{\prime}\right)$. Typical profiles of $\tilde{E}\left(p_{\hat{\lrcorner}}^{\prime}\right)$ were exemplified in Fig. 13 .

Utilizing the dressed fermion propagators, we then derived the quark-antiquark bound-state equation interpolating between IFD and LFD for the equal bare quark and antiquark mass $m$ and solved numerically the corresponding bound-state equations. From the numerical solutions of the spectroscopy, we find that the meson mass spectrum is independent of interpolation angle between the IFD and LFD as expected for physical observables. In particular, for the bare quark mass $m \rightarrow 0$, we confirmed the GOR behavior of the pionic ground-state mass square $\mathcal{M}_{(0)}^{2} \sim$ $m \sqrt{\lambda} \rightarrow 0$ as shown in Fig. 15. Our result is consistent with the discussions $[4,12,30,37]$ on the SBCS in the 't Hooft model $\left(N_{c} \rightarrow \infty\right)$. Plotting the meson mass spectra $\mathcal{M}_{(n)}$ $(n=0,1,2, \ldots, 7)$ for various $m$ values as summarized in Table VI, we also observe the Regge trajectory feature as shown in Fig. 16. The corresponding bound-state wave functions $\hat{\phi}_{ \pm}^{(n)}\left(r_{\lrcorner}, x\right)$ were obtained, in particular, for the low-lying states, i.e., $n=0$ and 1 states, and were applied to the interpolating formulation of "quasi-PDFs". The results of the wave functions clearly dictate the charge conjugation symmetry, exhibiting the symmetric and antisymmetric behaviors of $\hat{\phi}_{ \pm}^{(0)}\left(r_{\lrcorner}, x\right)$ and $\hat{\phi}_{ \pm}^{(1)}\left(r_{\lrcorner}, x\right)$, respectively, under the exchange of $x \leftrightarrow 1-x$. It is also interesting to note that the massless Goldstone boson cannot exist in the rest frame due to the null normalization from the equivalence between $\hat{\phi}_{+}^{(0)}\left(p_{\hat{\varkappa}}\right)$ and $\hat{\phi}_{-}^{(0)}\left(p_{\hat{\iota}}\right)$ for the massless ground-state in the rest frame as shown in Fig. 41.

Applying the bound-state wave functions $\hat{\phi}_{ \pm}^{(n)}\left(r_{\hat{\wedge}}, x\right)$ for the computation of the interpolating "quasi-PDFs" given by Eq. (132), we note the consistency with the observation made in Ref. [22] for the quasi-PDFs at $\delta=0$ (IFD) that there exists considerable difference between the shapes of the LFD result and the IFD quasi-PDF result for the light mesons. Our results indicate that the slow approach to the LFD-like results may be remedied by varying the interpolation parameter $\delta$ as well as the interpolating longitudinal meson momentum $r_{\star}$. For the future work, one may explore such idea to search for the "sweet spot" of $\delta$ and $r_{\text {A }}$ to attain most effective computation with the least sensitive numerical errors in getting the LFD result. Extending the Wick rotation technique to the interpolating time $x^{\hat{+}}$, the idea of searching for the "sweet spot" may be applicable to the usual lattice formulation in the Eunclidean space. This would be in good contrast to the recent application of the present interpolating formulation to the two-dimensional $\phi^{4}$ theory using the discretization technique in Minkowsky space consistent with the discrete light-cone quantization (DLCQ) approach $[20,44]$. It will be interesting to explore 
both "Euclidean" and "Minkowsky" numerical approaches implementing the interpolating formulation between IFD and LFD.

\section{ACKNOWLEDGMENTS}

This work was supported by the U.S. Department of Energy Grant No. DE-FG02-03ER41260. This research used resources of the National Energy Research Scientific Computing Center, which is supported by the Office of Science of the U.S. Department of Energy under Contract No. DE-AC02-05CH11231.

\section{APPENDIX A: BOGOLIUBOV TRANSFORMATION FOR THE INTERPOLATING SPINORS BETWEEN IFD AND LFD}

In this Appendix, we summarize the interpolating spinors and $\gamma$ matrices in the $1+1$ dimensional chiral representation and the Bogoliubov transformation between the free and interacting spinors as well as the corresponding creation/annihilation operators.

For the representation of the spinors [19], we adopt the chiral representation (CR), under which the usual $\gamma$ matrices in IFD for the $1+1$ dimensions are given by the Pauli matrices:

$$
\begin{aligned}
& \gamma^{0}=\sigma_{1}=\left(\begin{array}{ll}
0 & 1 \\
1 & 0
\end{array}\right), \quad \gamma^{1}=i \sigma_{2}=\left(\begin{array}{cc}
0 & 1 \\
-1 & 0
\end{array}\right), \\
& \gamma^{5}=\gamma_{5}=-\gamma_{0} \gamma_{1}=\gamma^{0} \gamma^{1}=-\sigma_{3}=\left(\begin{array}{cc}
-1 & 0 \\
0 & 1
\end{array}\right) .
\end{aligned}
$$

These can be transformed into standard representation (SR) easily by a transformation matrix

$$
S=S^{\dagger}=\frac{1}{\sqrt{2}}\left(\begin{array}{cc}
1 & 1 \\
1 & -1
\end{array}\right)
$$

through

$$
\gamma_{\mathrm{SR}}^{\mu}=S \gamma_{\mathrm{CR}}^{\mu} S^{\dagger} .
$$

In the SR representation, the free spinors in the rest frame in IFD are typically given by

$$
u_{\mathrm{SR}}^{(0)}\left(p^{1}=0\right)=\sqrt{2 m}\left(\begin{array}{l}
1 \\
0
\end{array}\right), \quad v_{\mathrm{SR}}^{(0)}\left(p^{1}=0\right)=\sqrt{2 m}\left(\begin{array}{l}
0 \\
1
\end{array}\right),
$$

where we take the normalization factor $\bar{u}_{\mathrm{SR}}^{(0)} u_{\mathrm{SR}}^{(0)}=2 m$ in conformity with the standard textbooks [24]. The corresponding free spinors in the chiral representation are used in this work without denoting the "CR" specification:

$$
\begin{aligned}
& u^{(0)}\left(p^{1}=0\right)=S \cdot u_{\mathrm{SR}}^{(0)}\left(p^{1}=0\right)=\sqrt{m}\left(\begin{array}{c}
1 \\
1
\end{array}\right), \\
& v^{(0)}\left(p^{1}=0\right)=S \cdot v_{\mathrm{SR}}^{(0)}\left(p^{1}=0\right)=\sqrt{m}\left(\begin{array}{c}
1 \\
-1
\end{array}\right) .
\end{aligned}
$$

The IFD spinors in the moving frame with the energy $E_{p}=$ $\sqrt{\left(p^{1}\right)^{2}+m^{2}}$ are then obtained as

$$
\begin{aligned}
u^{(0)}\left(p^{1}\right) & =B(\eta) u^{(0)}(0)=\sqrt{m}\left(\begin{array}{cc}
e^{-\frac{\eta}{2}} & 0 \\
0 & e^{\frac{\eta}{2}}
\end{array}\right)\left(\begin{array}{l}
1 \\
1
\end{array}\right) \\
& =\left(\begin{array}{c}
\sqrt{E_{p}-p^{1}} \\
\sqrt{E_{p}+p^{1}}
\end{array}\right),
\end{aligned}
$$

and

$$
v^{(0)}\left(-p^{1}\right)=B(-\eta) v^{(0)}(0)=\left(\begin{array}{c}
\sqrt{E_{p}+p^{1}} \\
-\sqrt{E_{p}-p^{1}}
\end{array}\right),
$$

where the usual boost operator $B(\eta)$ with the rapidity $\eta=\tanh ^{-1} \frac{p^{1}}{E_{p}}$ and the longitudinal boost generator $K^{1}$ is given by

$B(\eta)=\exp \left(-i \eta \cdot K^{1}\right)=\exp \left(-\frac{1}{2} \eta \sigma_{3}\right)=\exp \left(\frac{1}{2} \eta \gamma_{5}\right)$.

In terms of the interpolating momentum variables, the rapidity $\eta$ can be written [19] as

$$
\eta=\log \left(\frac{p^{\hat{+}}+p_{\hat{\imath}}}{m(\cos \delta+\sin \delta)}\right)
$$

where one can note the following equality as well

$$
-\eta=\log \left(\frac{p^{\hat{+}}-p_{\text {合 }}}{m(\cos \delta-\sin \delta)}\right) .
$$

The boost operator $B(\eta)$ can then be written in terms of the interpolating momentum variables as

$$
B(\eta)=\left(\begin{array}{cc}
\sqrt{\frac{p^{+}-p_{\lrcorner}}{m(\cos \delta-\sin \delta)}} & 0 \\
0 & \sqrt{\frac{p^{+}+p_{\lrcorner}}{m(\cos \delta+\sin \delta)}}
\end{array}\right),
$$

so that the boosted interpolating spinors are given by 


$$
u^{(0)}\left(p_{\hat{\sim}}\right)=\left(\begin{array}{c}
\sqrt{\frac{p^{\hat{f}}-p_{\hat{\iota}}}{\cos \delta-\sin \delta}} \\
\sqrt{\frac{p^{\hat{f}}+p_{\hat{\iota}}}{\cos \delta+\sin \delta}}
\end{array}\right),
$$

and

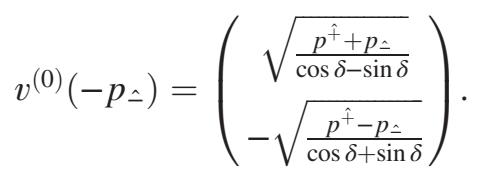

Although Eqs. (A12)-(A13) are expressed in terms of the interpolating momentum variables while Eqs. (A6)-(A7) are written in terms of the IFD momentum variables, one should note that they are intrinsically the same spinors with respect to each other as we have shown in the above derivation. As $\delta \rightarrow \frac{\pi}{4}$, i.e., in the LFD limit, Eqs. (A12)(A13) coincide with the LFD spinors [19]

$$
u^{(0)}\left(p^{+}\right)=\frac{1}{\sqrt{\sqrt{2} p^{+}}}\left(\begin{array}{c}
m \\
\sqrt{2} p^{+}
\end{array}\right),
$$

and

$$
v^{(0)}\left(p^{+}\right)=\frac{1}{\sqrt{\sqrt{2} p^{+}}}\left(\begin{array}{c}
m \\
-\sqrt{2} p^{+}
\end{array}\right),
$$

where one may note the correspondence

$$
\frac{p^{\hat{+}}-p_{\text {二 }}}{\mathbb{C}} \stackrel{\mathbb{C} \rightarrow 0}{\rightarrow} \frac{m^{2}}{2 p^{+}},
$$

with $p^{\hat{+}} \rightarrow p^{+}$, and $p_{\hat{\imath}} \rightarrow p^{+}$. Thus, the interpolating spinors given by Eqs. (A12)-(A13) are nothing but the same spinors as given by Eqs. (A6)-(A7) in IFD and Eqs. (A14)-(A15) in LFD, respectively. There are no differences in the spinors except the expression difference in terms of the momentum variables taken in each different form of the dynamics.

Dropping the "CR" specification again for the $\gamma$ matrices, we follow the link given by Eq. (1) to get the following interpolating $\gamma$ matrices in the chiral representation as

$$
\begin{aligned}
& \gamma^{\hat{+}}=\left(\begin{array}{cc}
0 & \sqrt{1+\mathbb{S}} \\
\sqrt{1-\mathbb{S}} & 0
\end{array}\right), \\
& \gamma^{\hat{\imath}}=\left(\begin{array}{cc}
0 & -\sqrt{1-\mathbb{S}} \\
\sqrt{1+\mathbb{S}} & 0
\end{array}\right) .
\end{aligned}
$$

Lowering the indices with the interpolating metric given by Eq. (2), we also get

$$
\begin{aligned}
& \gamma_{\hat{+}}=\mathbb{C} \gamma^{\hat{+}}+\mathbb{S} \gamma^{\hat{\imath}}=\left(\begin{array}{cc}
0 & \sqrt{1-\mathbb{S}} \\
\sqrt{1+\mathbb{S}} & 0
\end{array}\right), \\
& \gamma_{\hat{\imath}}=\mathbb{S} \gamma^{\hat{+}}-\mathbb{C} \gamma^{\hat{\imath}}=\left(\begin{array}{cc}
0 & \sqrt{1+\mathbb{S}} \\
-\sqrt{1-\mathbb{S}} & 0
\end{array}\right),
\end{aligned}
$$

where $\sqrt{1 \pm \mathbb{S}}$ can be identically given by $\cos \delta \pm \sin \delta$ with $\cos \delta \geq \sin \delta$ always due to $0 \leq \delta \leq \frac{\pi}{4}$. One can explicitly check that the interpolating $\gamma$ matrices satisfy $\left\{\gamma^{\hat{\mu}}, \gamma^{\hat{\nu}}\right\}=2 g^{\hat{\mu} \hat{\nu}} \cdot \mathbf{I}_{2 \times 2}$, in particular,

$\left(\gamma^{\hat{+}}\right)^{2}=\left(\gamma_{\hat{+}}\right)^{2}=\mathbb{C} \cdot \mathbf{I}_{2 \times 2}, \quad\left(\gamma^{\hat{4}}\right)^{2}=\left(\gamma_{\hat{\epsilon}}\right)^{2}=-\mathbb{C} \cdot \mathbf{I}_{2 \times 2}$.

As $\delta \rightarrow \pi / 4$, one gets the usual LFD $\gamma$ matrices given by

$$
\begin{aligned}
& \gamma^{+}=\left(\gamma^{0}+\gamma^{1}\right) / \sqrt{2}=\left(\begin{array}{cc}
0 & \sqrt{2} \\
0 & 0
\end{array}\right)=\gamma_{-}, \\
& \gamma^{-}=\left(\gamma^{0}-\gamma^{1}\right) / \sqrt{2}=\left(\begin{array}{cc}
0 & 0 \\
\sqrt{2} & 0
\end{array}\right)=\gamma_{+},
\end{aligned}
$$

where $\left\{\gamma^{\mu}, \gamma^{\nu}\right\}=2 g^{\mu \nu} \cdot \mathbf{I}_{2 \times 2}$ and $\left(\gamma^{+}\right)^{2}=\left(\gamma^{-}\right)^{2}=0$.

Now, using the Bogoliubov transformation given by Eq. (23) for the creation/annihilation operators of the quark/ anti-quark fields as well as the boost operation for the free quark/antiquark fields, we get the following relationship between the interacting spinors and the free spinors at rest:

$u\left(p_{\hat{\varkappa}}\right)=T\left(p_{\hat{\varkappa}}\right) u^{(0)}(0), \quad v\left(-p_{\text {今 }}\right)=T\left(p_{\hat{\varkappa}}\right) v^{(0)}(0)$,

where

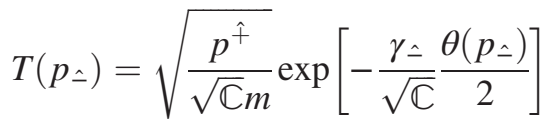

$$
\begin{aligned}
& =\sqrt{\frac{p^{\hat{+}}}{\sqrt{\mathbb{C}} m}} \exp \left[-\frac{\gamma_{\hat{\iota}}}{\sqrt{\mathbb{C}}} \frac{\theta_{f}\left(p_{\lrcorner}\right)+2 \zeta\left(p_{\lrcorner}\right)}{2}\right] \\
& =T_{f}\left(p_{\hat{\iota}}\right) \exp \left[-\frac{\gamma_{\hat{\imath}}}{\sqrt{\mathbb{C}}} \zeta\left(p_{\hat{\iota}}\right)\right]
\end{aligned}
$$

with

$$
\begin{aligned}
& \exp \left[-\frac{\gamma_{\hat{\iota}}}{\sqrt{\mathbb{C}}} \zeta\left(p_{\hat{\lrcorner}}\right)\right]=\cos \zeta\left(p_{\hat{\lrcorner}}\right) \cdot \mathbf{I}_{2 \times 2}-\sin \zeta\left(p_{\hat{\lrcorner}}\right) \frac{\gamma_{\hat{\iota}}}{\sqrt{\mathbb{C}}} \\
& =\left(\begin{array}{cc}
\cos \zeta\left(p_{\lrcorner}\right) & -\sin \zeta\left(p_{\lrcorner}\right) \sqrt{\frac{1+\mathbb{S}}{\mathbb{C}}} \\
\sin \zeta\left(p_{\lrcorner}\right) \sqrt{\frac{1-\mathbb{S}}{\mathbb{C}}} & \cos \zeta\left(p_{\lrcorner}\right)
\end{array}\right),
\end{aligned}
$$


and

$$
T_{f}\left(p_{\hat{\varkappa}}\right)=\sqrt{\frac{p^{\hat{+}}}{\sqrt{\mathbb{C}} m}} \exp \left[-\frac{\gamma_{\hat{\varkappa}}}{\sqrt{\mathbb{C}}} \frac{\theta_{f}\left(p_{\hat{\varkappa}}\right)}{2}\right]=\sqrt{\frac{p^{\hat{+}}}{\sqrt{\mathbb{C}} m}}\left(\cos \frac{\theta_{f}\left(p_{\hat{\varkappa}}\right)}{2} \cdot \mathbf{I}_{2 \times 2}-\sin \frac{\theta_{f}\left(p_{\hat{\varkappa}}\right)}{2} \cdot \frac{\gamma_{\hat{\varkappa}}}{\sqrt{\mathbb{C}}}\right) .
$$

Note here that the free spinors in the moving frame are related to each other as $\frac{\gamma_{\varkappa}}{\sqrt{\mathbb{C}}} \cdot u^{(0)}\left(p_{\hat{\iota}}\right)=v^{(0)}\left(-p_{\hat{\iota}}\right)$ and $\frac{\gamma_{\hat{\iota}}}{\sqrt{\mathbb{C}}} \cdot v^{(0)}\left(-p_{\hat{\iota}}\right)=-u^{(0)}\left(p_{\hat{\iota}}\right)$, and that the free spinors in the moving frame and the rest frame are related to each other

$$
\begin{aligned}
& u^{(0)}\left(p_{\hat{\iota}}\right)=\sqrt{\frac{p^{\hat{+}}}{\sqrt{\mathbb{C}} m}} \exp \left[-\frac{\gamma_{\hat{\iota}}}{\sqrt{\mathbb{C}}} \frac{\theta_{f}\left(p_{\hat{\iota}}\right)}{2}\right] u^{(0)}(0), \\
& v^{(0)}\left(-p_{\lrcorner}\right)=\sqrt{\frac{p^{\hat{+}}}{\sqrt{\mathbb{C}} m}} \exp \left[-\frac{\gamma_{\hat{\iota}}}{\sqrt{\mathbb{C}}} \frac{\theta_{f}\left(p_{\iota}\right)}{2}\right] v^{(0)}(0),
\end{aligned}
$$

where $\theta_{f}\left(p_{\hat{\varkappa}}\right)$ is given by Eq. (27) and is related in the IFD case to the rapidity $\eta \operatorname{as} \sin \theta_{f}=\tanh \eta$, while in general they are related by $\tanh \eta=\frac{\sin \theta_{f}-\tan \delta}{1-\sin \theta_{f} \tan \delta}$, or in other words, $\sin \theta_{f}=\frac{\tanh \eta+\tan \delta}{1+\tan \delta \tanh \eta}$. From Eq. (A21), one can get the interacting quark/ antiquark spinors given by Eqs. (28) and (29) in Sec. II A as well as Eqs. (45) and (46).

\section{APPENDIX B: MINIMIZATION OF THE VACUUM ENERGY WITH RESPECT TO THE BOGOLIUBOV ANGLE}

In this Appendix, we show that the mass gap equation given by Eq. (51) can also be obtained by minimizing the vacuum energy $\mathcal{E}_{v}$ in Eq. (37) with respect to the Bogoliubov angle, $\theta\left(p_{\iota}\right)$ in addition to the methods presented in Sec. II. Recall that

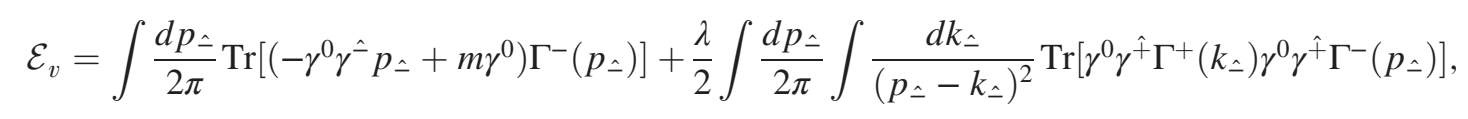

where $\Gamma^{ \pm}$is defined by Eqs. (45) and (46). Let us now compute the small variation of $\mathcal{E}_{v}$ as

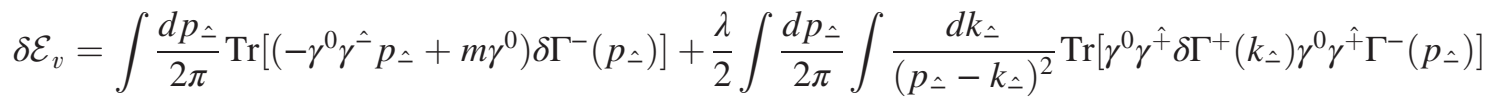

$$
\begin{aligned}
& +\frac{\lambda}{2} \int \frac{d p_{\hat{\varkappa}}}{2 \pi} \int \frac{d k_{\hat{\varkappa}}}{\left(p_{\hat{\iota}}-k_{\hat{\varkappa}}\right)^{2}} \operatorname{Tr}\left[\gamma^{0} \gamma^{\hat{+}} \Gamma^{+}\left(k_{\hat{\varkappa}}\right) \gamma^{0} \gamma^{\hat{+}} \delta \Gamma^{-}\left(p_{\hat{\varkappa}}\right)\right] \text {. }
\end{aligned}
$$

In the second term of the above equation, we are able to swap variables $p_{\varkappa}$ and $k_{\varkappa}$, i.e.,

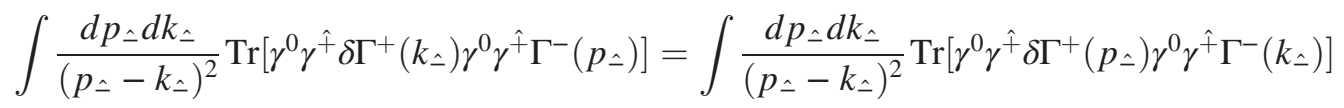

$$
\begin{aligned}
& =-\int \frac{d p_{\hat{\iota}} d k_{\varkappa}}{\left(p_{\hat{\iota}}-k_{\hat{\iota}}\right)^{2}} \operatorname{Tr}\left[\gamma^{0} \gamma^{\hat{+}} \delta \Gamma^{-}\left(p_{\hat{\iota}}\right) \gamma^{0} \gamma^{\hat{+}} \Gamma^{-}\left(k_{\hat{\iota}}\right)\right],
\end{aligned}
$$

where we used the fact that the sum of $\Gamma^{+}\left(p_{\hat{\varkappa}}\right)$ and $\Gamma^{-}\left(p_{\hat{\varkappa}}\right)$ is independent of $p_{\hat{\iota}}$ from the first equality to the second equality. Thus, we obtain

$$
\begin{aligned}
& \delta \mathcal{E}_{v}=\int \frac{d p_{\hat{\varkappa}}}{2 \pi} \operatorname{Tr}\left[\left(-\gamma^{0} \gamma^{\hat{\imath}} p_{\hat{\iota}}+m \gamma^{0}\right) \delta \Gamma^{-}\left(p_{\hat{\iota}}\right)\right]
\end{aligned}
$$

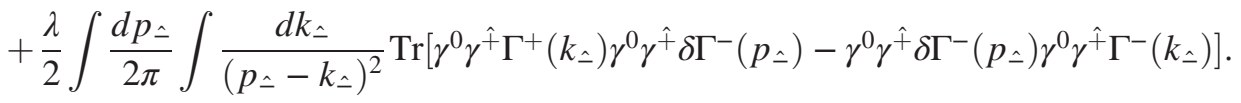

The functional differentiation of $\mathcal{E}_{v}$ relative to $\theta\left(p_{\wedge}\right)$ for a given $p_{\wedge}$ is then given by 


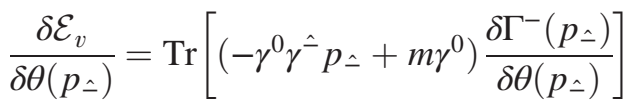

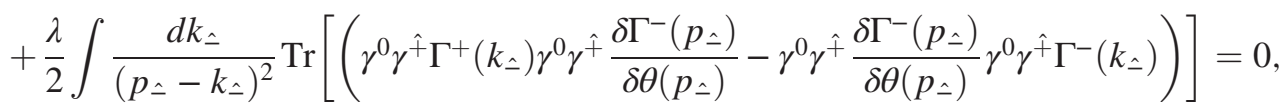

i.e.,

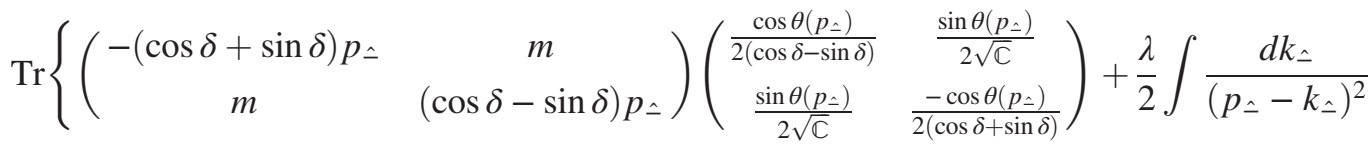

$$
\begin{aligned}
& \times\left[\left(\begin{array}{cc}
\frac{1-\sin \theta\left(k_{\lrcorner}\right)}{2} & \frac{\cos \delta-\sin \delta}{2 \sqrt{\complement}} \cos \theta\left(k_{\lrcorner}\right) \\
\frac{\cos \delta+\sin \delta}{2 \sqrt{\complement}} \cos \theta\left(k_{\lrcorner}\right) & \frac{1+\sin \theta\left(k_{\lrcorner}\right)}{2}
\end{array}\right)\left(\begin{array}{cc}
\frac{\cos \theta\left(p_{\lrcorner}\right)}{2} & \frac{(\cos \delta-\sin \delta)}{2 \sqrt{\complement}} \sin \theta\left(p_{\lrcorner}\right) \\
\frac{(\cos \delta+\sin \delta)}{2 \sqrt{\complement}} \sin \theta\left(p_{\lrcorner}\right) & \frac{-\cos \theta\left(p_{\lrcorner}\right)}{2}
\end{array}\right)\right.
\end{aligned}
$$

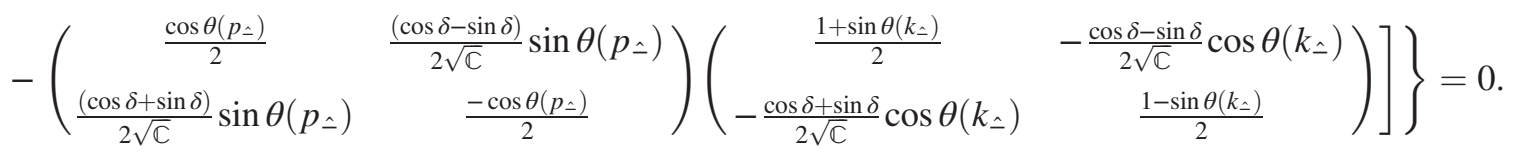

The computation of the trace leads to the gap equation given by Eq. (51).

\section{APPENDIX C: TREATMENT OF THE $\lambda=0$ (FREE) CASE VS THE $\lambda \neq 0$ (INTERACTING) CASE WITH RESPECT TO THE MASS DIMENSION $\sqrt{\mathbf{2} \lambda}$}

As the 't Hooft coupling $\lambda$ given by Eq. (35) has the mass-square dimension, we scaled out the mass dimension $\sqrt{2 \lambda}$ and used the dimensionless mass $m$, longitudinal momentum $p_{\text {^ }}$, etc., in presenting all the figures and tables of our work. Of course, we could have explicitly defined the dimensionless variables, e.g., denoted by

$$
\bar{p}_{\hat{\iota}}=\frac{p_{\hat{\iota}}}{\sqrt{2 \lambda}}, \quad \bar{E}=\frac{E}{\sqrt{2 \lambda}}, \quad \bar{m}=\frac{m}{\sqrt{2 \lambda}},
$$

and rewrite, for example, Eqs. (48) and (51) as

$$
\begin{aligned}
\bar{E}\left(\bar{p}_{\hat{\iota}}\right)= & \bar{p}_{\hat{\iota}} \sin \theta\left(\bar{p}_{\hat{\iota}}\right)+\sqrt{\mathbb{C}} \bar{m} \cos \theta\left(\bar{p}_{\hat{\iota}}\right) \\
& +\mathbb{C} \cdot \frac{1}{4} f \frac{d \bar{k}_{\hat{\iota}}}{\left(\bar{p}_{\hat{\iota}}-\bar{k}_{\hat{\iota}}\right)^{2}} \cos \left(\theta\left(\bar{p}_{\hat{\iota}}\right)-\theta\left(\bar{k}_{\hat{\iota}}\right)\right),
\end{aligned}
$$

and

$$
\begin{aligned}
& \bar{p}_{\lrcorner} \cos \theta\left(\bar{p}_{\hat{\lrcorner}}\right)-\sqrt{\mathbb{C}} \bar{m} \sin \theta\left(\bar{p}_{\hat{\iota}}\right) \\
& \quad=\mathbb{C} \cdot \frac{1}{4} f \frac{d \bar{k}_{\hat{\iota}}}{\left(\bar{p}_{\hat{\iota}}-\bar{k}_{\hat{\iota}}\right)^{2}} \sin \left(\theta\left(\bar{p}_{\hat{\iota}}\right)-\theta\left(\bar{k}_{\hat{\iota}}\right)\right),
\end{aligned}
$$

respectively. Similarly, we can also scale out the interpolation angle dependence by defining the following rescaled variables

$$
\bar{p}_{\hat{\iota}}^{\prime}=\frac{\bar{p}_{\hat{\wedge}}}{\sqrt{\mathbb{C}}}, \quad \bar{E}^{\prime}=\frac{\bar{E}}{\sqrt{\mathbb{C}}},
$$

to reduce Eqs. (C2) and (C3) as

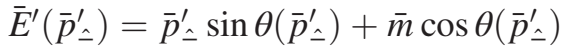

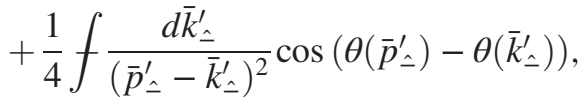

and

$$
\begin{aligned}
& \bar{p}_{\varkappa}^{\prime} \cos \theta\left(\bar{p}_{\varkappa}^{\prime}\right)-\bar{m} \sin \theta\left(\bar{p}_{\varkappa}^{\prime}\right)
\end{aligned}
$$

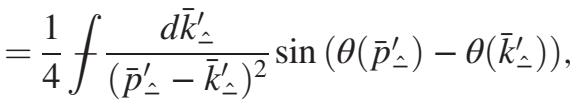

respectively. Indeed, we used such rescaled variables in Eqs. (83), (95) and (96) to present the corresponding results without any dependence of $\delta \in[0, \pi / 4]$, confirming that the physical results are indeed invariant regardless of the interpolation angle $\delta$ as they must be. However, one should note the contrast between the scaling by the dimensionful parameter $\sqrt{2 \lambda}$ and the scaling by the dimensionless parameter $\sqrt{\mathbb{C}}$. While the rescaling over the dimensionless variable $\mathbb{C}$ includes the limit of $\mathbb{C}=0$, i.e., LFD, the rescaling over the dimensionful variable $\sqrt{2 \lambda}$ cannot include the limit to $\lambda=0$. Namely, the free theory without any interaction must be distinguished from the interacting theory and should be discussed separately. For $\lambda=0$, in fact, the mass gap solution $\theta\left(p_{\wedge}\right)=\theta_{f}\left(p_{\wedge}\right)$ can be immediately found even analytically by taking the righthand side of Eq. (51) to be zero, i.e., 


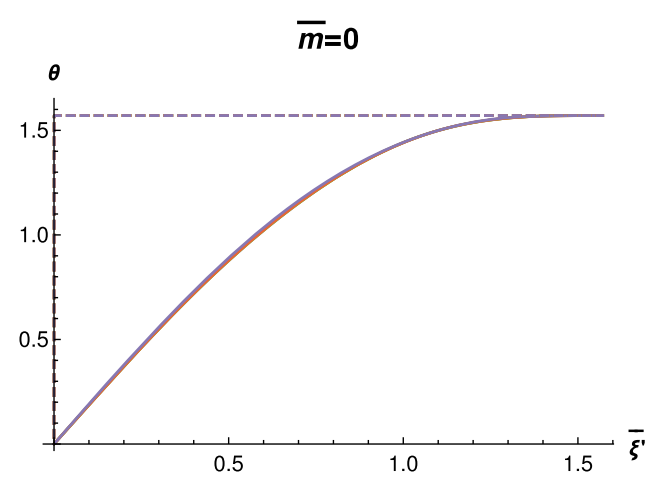

(a)

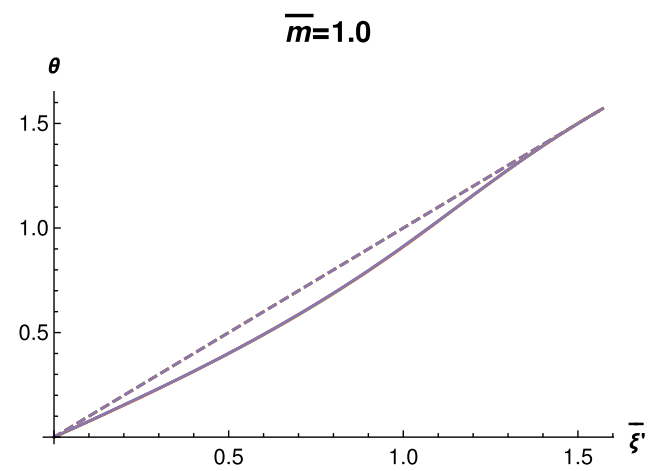

(c)

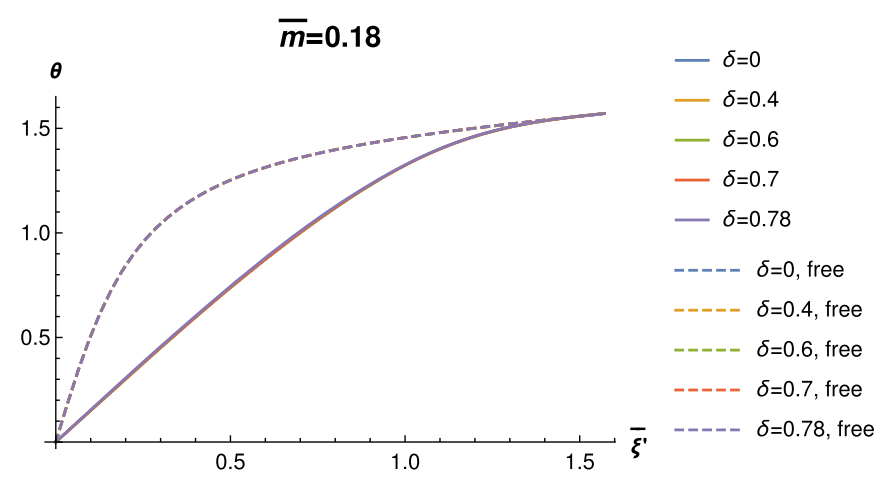

(b)

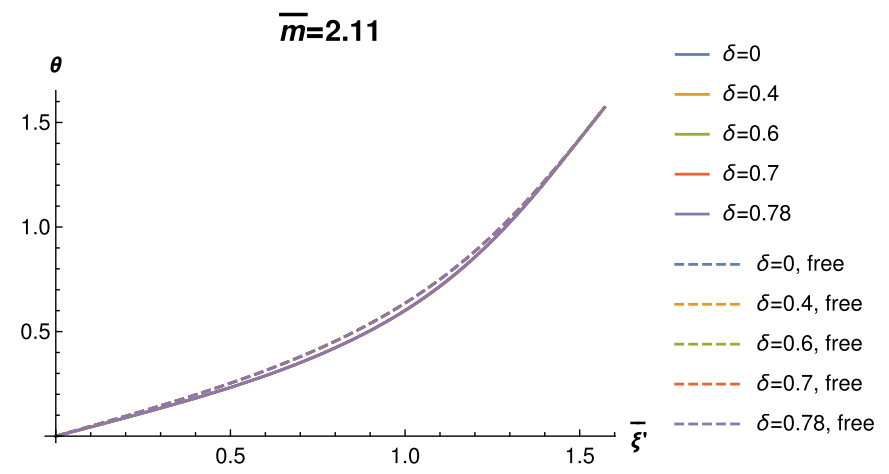

(d)

FIG. 28. The numerical solutions of $\theta\left(\bar{p}_{\hat{\Lambda}}^{\prime}\right)$ versus the free solutions $\theta_{f}\left(\bar{p}_{\hat{\prime}}^{\prime}\right)$ for a few choices of quark mass (a) $\bar{m}=0$ (b) $\bar{m}=0.18$ (c) $\bar{m}=1.0$ and (d) $\bar{m}=2.11$, for several different interpolation angles.

$$
\theta_{f}\left(p_{\hat{\iota}}\right)=\arctan \left(\frac{p_{\hat{\iota}}}{\sqrt{\mathbb{C} m}}\right)
$$

where the dimensionless ratio $p_{\wedge} / m$ can still be written as $\bar{p}_{-} / \bar{m}$ with the cancellation of the $\lambda=0$ factor in the ratio. In terms of the rescaled variables in Eqs. (C1) and (C4), this analytic free solution becomes

$$
\theta_{f}\left(\bar{p}_{\hat{\prime}}^{\prime}\right)=\arctan \left(\frac{\bar{p}_{\hat{\iota}}^{\prime}}{\bar{m}}\right)
$$

In Fig. 28, we plot the interacting mass gap solution $\theta\left(\bar{p}_{-}^{\prime}\right)$ in comparison with the free mass gap solution $\theta_{f}\left(\bar{p}_{\hat{\prime}}^{\prime}\right)$ as functions of rescaled variable $\bar{\xi}^{\prime}=\tan ^{-1} \bar{p}_{\hat{.}}^{\prime}$. Note here that the interacting mass gap solution $\theta\left(\bar{p}_{\dot{A}}^{\prime}\right)$ includes the LFD solution analytically given by Eq. (67) while the free analytic solution $\theta_{f}\left(\bar{p}_{-}^{\prime}\right)$ is clearly distinguished from the interacting solution $\theta\left(\bar{p}_{\lrcorner}^{\prime}\right)$, although the difference between the free solution and the interacting solution gets reduced as $\bar{m}$ gets larger. It confirms that the entire nontrivial contributions from the interaction to the LFD solution are provided by the zero-mode $p^{+}=0$ as the finite $\bar{p}_{\hat{\iota}}^{\prime}$ can be attained only if $\bar{p}_{\hat{\iota}}=p^{+} / \sqrt{2 \lambda}=0$ in the limit $\sqrt{\mathbb{C}} \rightarrow 0$ (LFD).

\section{APPENDIX D: MESONIC WAVE FUNCTIONS FOR $m=0.045,1.0$, AND 2.11 IN THE UNIT OF $\sqrt{2 \lambda}$}

In Sec. VIB, we discussed the wave functions of the quark-antiquark bound states for the cases of $m=0$ and $m=0.18$ in the unit of $\sqrt{2 \lambda}$, i.e., $\bar{m}=0$ and 0.18 . In this Appendix, we summarize the numerical results of $\hat{\phi}_{ \pm}^{(0)}\left(r_{\lrcorner}, x\right)$ and $\hat{\phi}_{ \pm}^{(1)}\left(r_{\lrcorner}, x\right)$ for a few other bare quark/ antiquark mass cases; $m=0.045,1.0$ and 2.11 in the unit of $\sqrt{2 \lambda}$, i.e., $\bar{m}=0.045,1.0$ and 2.11. In particular, $\bar{m}=$ 0.045 corresponds to the physical pion mass $M_{\pi}=0.41$ in the unit of $\sqrt{2 \lambda}$ according to the reasoning [12] mentioned in Sec. III. We also note that the free mass gap solution $\theta_{f}\left(\bar{p}_{\wedge}^{\prime}\right)$ given by Eq. (C8) for $\bar{m}=1.0$ exhibits the straight line profile in the plot with respect to $\bar{\xi}^{\prime}=\tan ^{-1} \bar{p}_{-}^{\prime}$ while the profile of the solution for $\bar{m}>1.0$, e.g., $\bar{m}=2.11$, gets bent toward the concave shaped profile from the convex shaped profile for $\bar{m}<1.0$ as one can see in Fig. 28 . 


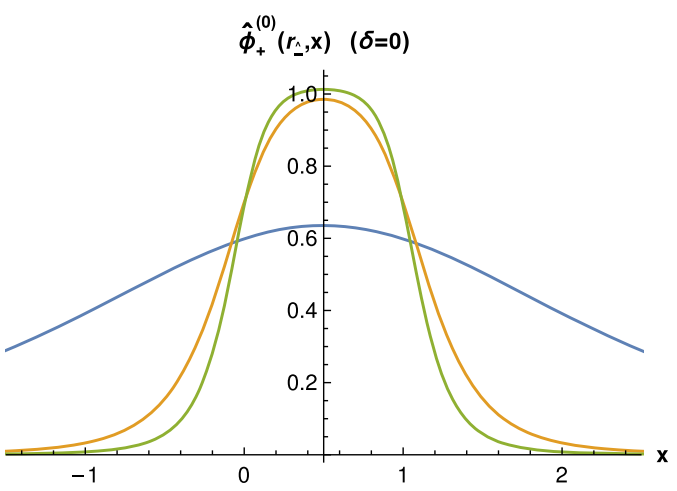

(a)

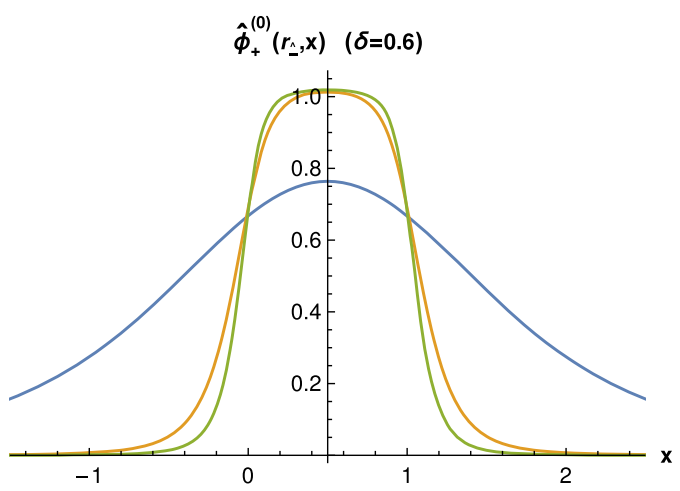

(c)

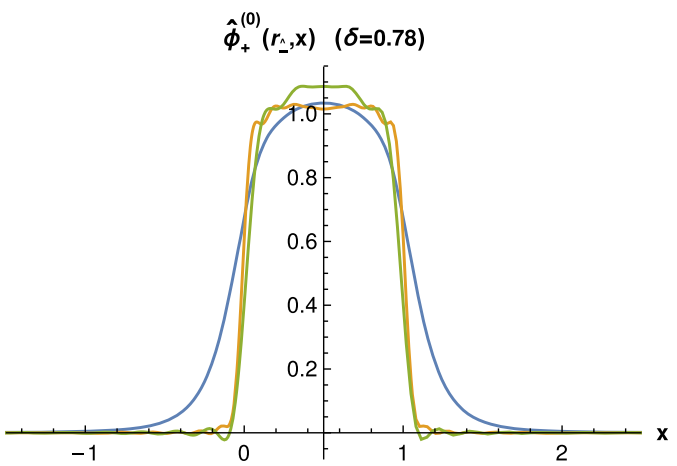

(e)

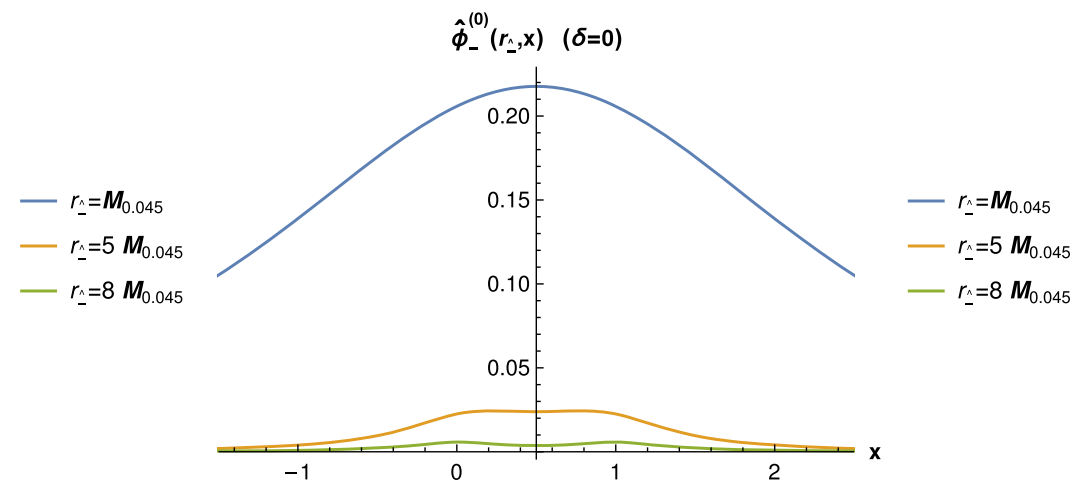

(b)

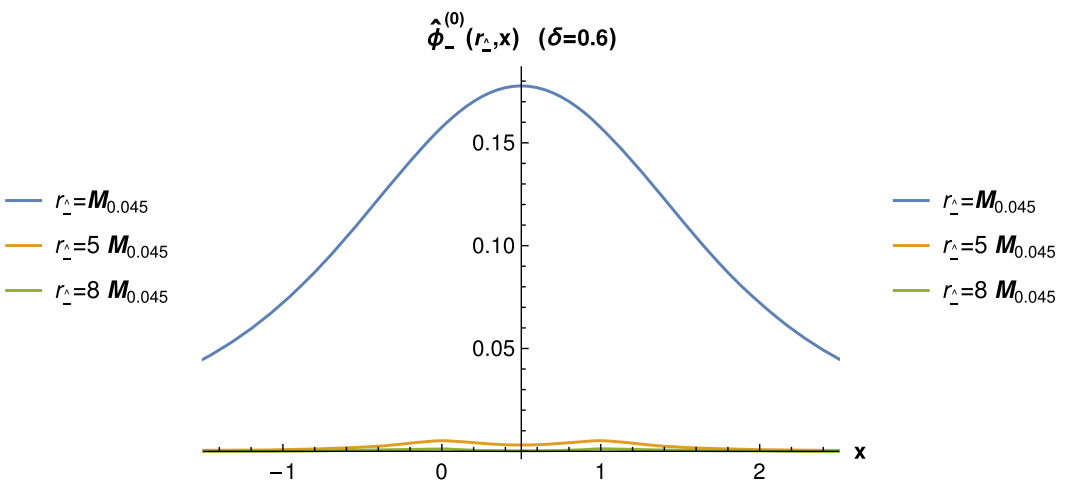

(d)
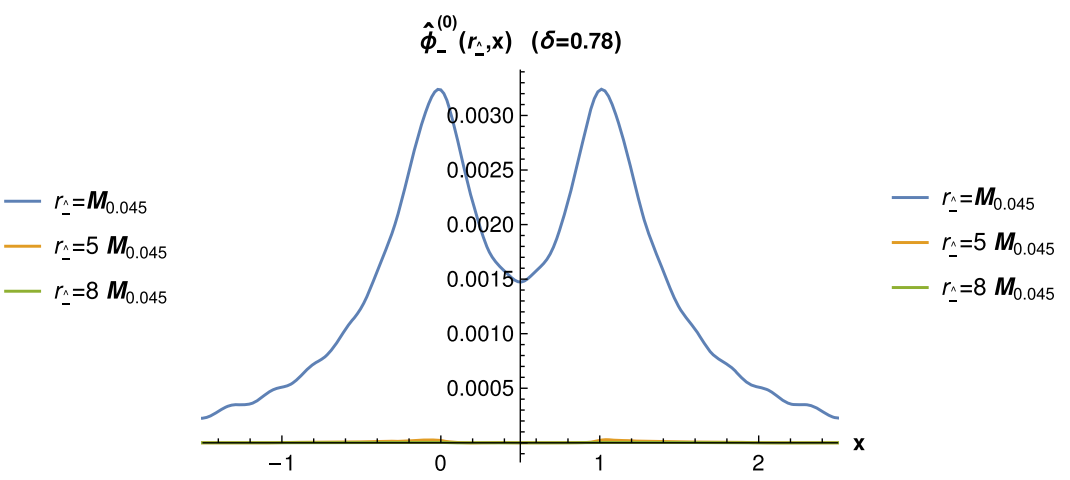

(f)

FIG. 29. Ground state wave functions $\hat{\phi}_{+}^{(0)}\left(r_{\hat{\iota}}, x\right)$ and $\hat{\phi}_{-}^{(0)}\left(r_{\hat{\iota}}, x\right)$ for $m=0.045$. All quantities are in proper units of $\sqrt{2 \lambda}$.

For the case of $\bar{m}=0.045$, the numerical results of $\hat{\phi}_{ \pm}^{(0)}\left(r_{\lrcorner}, x\right)$ and $\hat{\phi}_{ \pm}^{(1)}\left(r_{\lrcorner}, x\right)$ are presented in Figs. 29 and 30 , respectively. In each figure, the results of $\delta=0,0.6$ and 0.78 are shown in the top, middle and bottom panels, respectively. In each panel, the results of $r_{\hat{\imath}}=\mathbf{M}_{0.045}$, $5 \mathbf{M}_{0.045}$ and $8 \mathbf{M}_{0.045}$, where $\mathbf{M}_{0.045}=0.42$ (see Table VI) is the ground state meson mass for the quark mass value $\bar{m}=0.045$, are depicted by the solid lines in blue, yellow, and green, respectively. We note that the $\operatorname{IFD}(\delta=0)$ results shown in Figs. 29(a), 29(b), 30(a), and 30(b) coincide with the corresponding plots in Fig. 9 of Ref. [12]. The results for the case of $\bar{m}=0.045$ exhibit the similar features that we discussed for the case of $\bar{m}=0$ in Sec. VI B. Namely, the large-momentum IFD $(\delta=0)$ numerical results approach to the LFD results quite slowly [22] as the momenta $r_{-}$get large [see Figs. 29(a), 29(b), 30(a), and 30(b)], while the results getting close to $\delta=\pi / 4$ (e.g., $\delta=0.78$ ) yield very quickly the essential features of the LFD results fitting in the region 


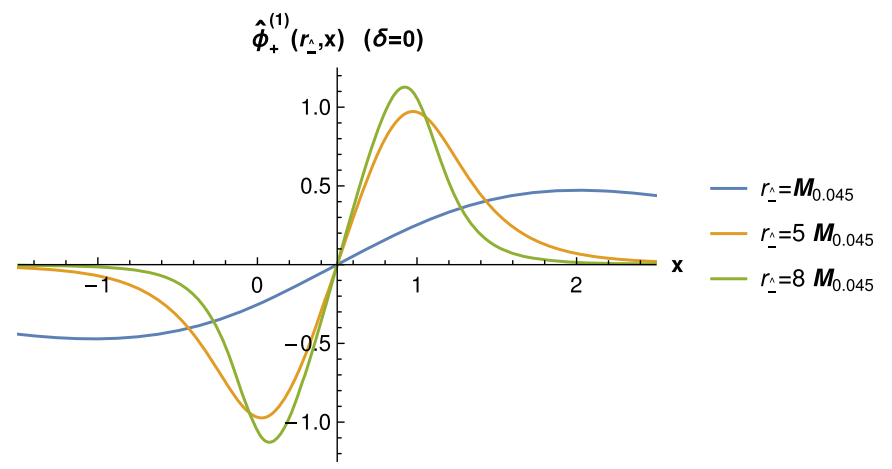

(a)

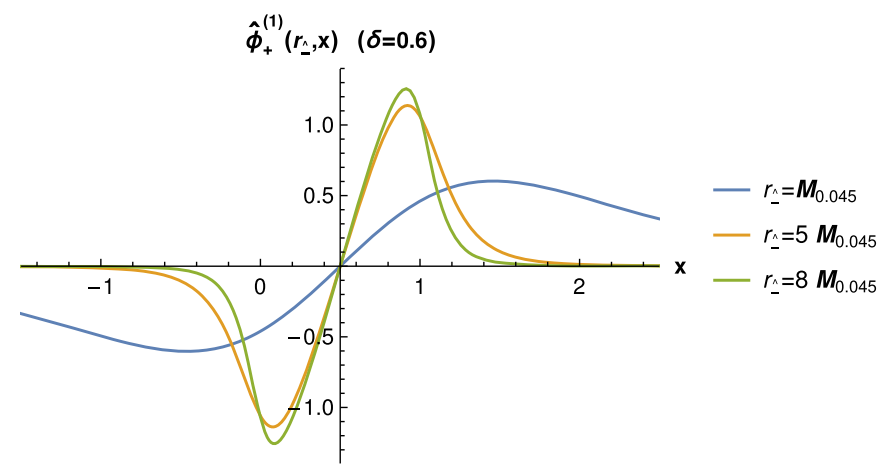

(c)

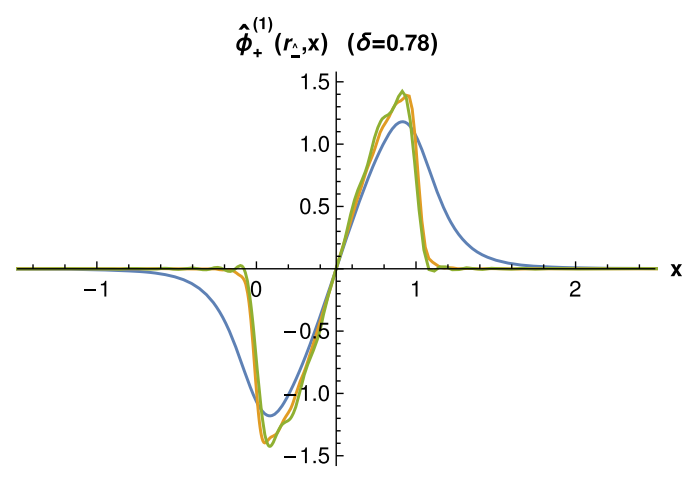

(e)

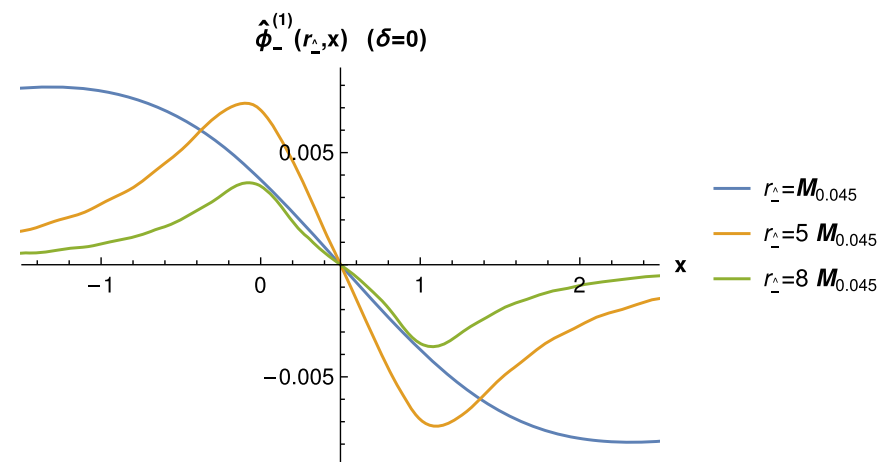

(b)

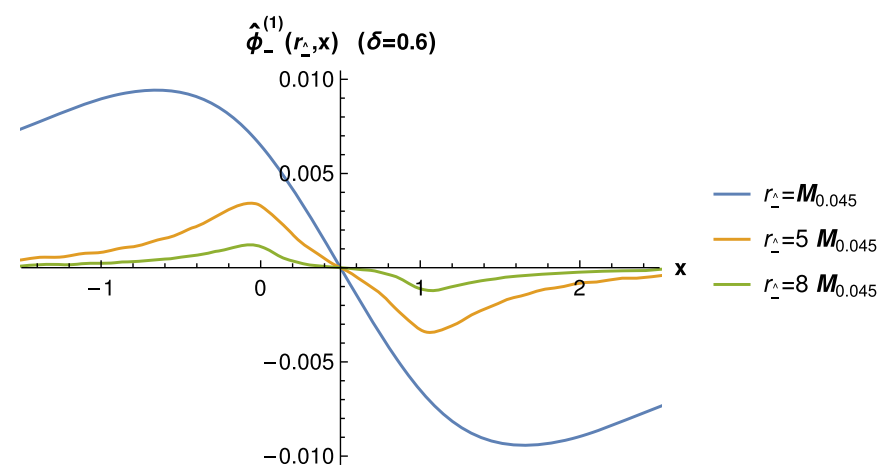

(d)

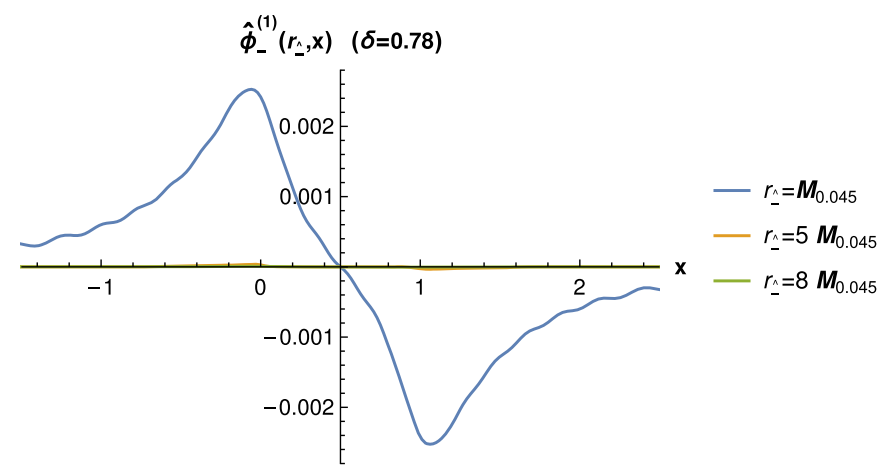

(f)

FIG. 30. First excited state wave functions $\hat{\phi}_{+}^{(1)}\left(r_{\hat{\iota}}, x\right)$ and $\hat{\phi}_{-}^{(1)}\left(r_{\hat{\imath}}, x\right)$ for $m=0.045$. All quantities are in proper units of $\sqrt{2 \lambda}$.

$[0,1]$ regardless of the momenta $r_{\hat{\varkappa}}=\mathbf{M}_{0.045}, 5 \mathbf{M}_{0.045}$, or $8 \mathbf{M}_{0.045}$ and the minus component disappears [see Figs. 29(e), 29(f), 30(e), and 30(f)] although the numerical sensitivity gets enhanced with some wiggles or bulges in $\hat{\phi}_{+}^{(0)}\left(r_{\hat{\imath}}, x\right)(\delta=0.78)$ for $r_{\hat{\imath}}=5 \mathbf{M}_{0.045}$ and $8 \mathbf{M}_{0.045}$ due to the enhanced demand of numerical accuracy as $\mathbb{C}$ gets close to zero and $r_{\text {^ }}$ gets large. In Figs. 29 and 30, the charge conjugation symmetry under the exchange of $x \leftrightarrow 1-x$ is manifest as we have discussed for the ground state and the first excited state previously in Sec. VIB, i.e., $\hat{\phi}_{+}^{(1)}\left(r_{\lrcorner}, x\right)$ and $\hat{\phi}_{-}^{(1)}\left(r_{-}, x\right)$ reveal the antisymmetric profiles while $\hat{\phi}_{+}^{(0)}\left(r_{\hat{\iota}}, x\right)$ and $\hat{\phi}_{-}^{(0)}\left(r_{\lrcorner}, x\right)$ exhibit the symmetric profiles.

For the case of $\bar{m}=1.0$ which we noted above its straight line profile for $\theta_{f}\left(\bar{p}_{\hat{A}}^{\prime}\right)$ in Fig. 28, the numerical results of $\hat{\phi}_{ \pm}^{(0)}\left(r_{\hat{\iota}}, x\right)$ and $\hat{\phi}_{ \pm}^{(1)}\left(r_{\hat{\iota}}, x\right)$ are presented in Figs. 31 and 32, respectively. The frames for $\bar{m}=1.0$ are chosen as $r_{\imath}=0.2 \mathbf{M}_{1.0}, 2 \mathbf{M}_{1.0}$, and $5 \mathbf{M}_{1.0}$, where 


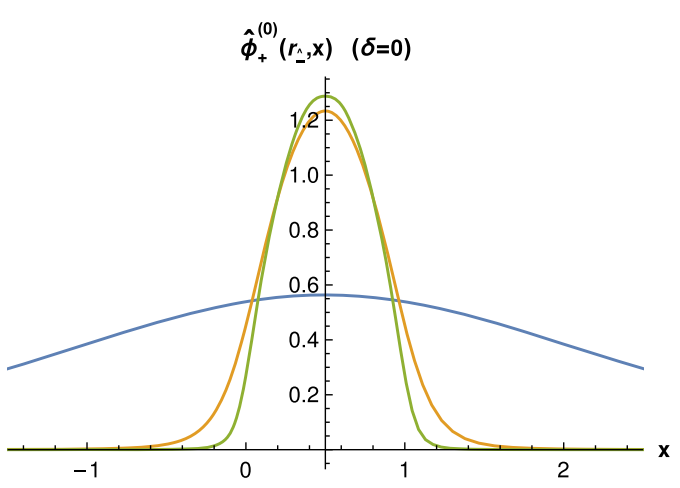

(a)

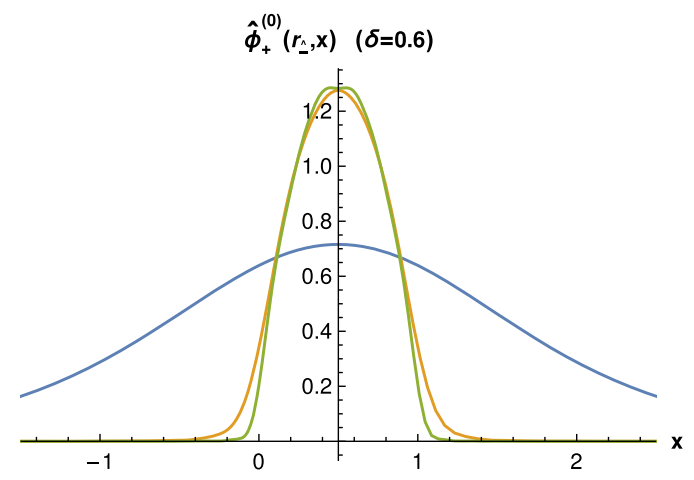

(c)

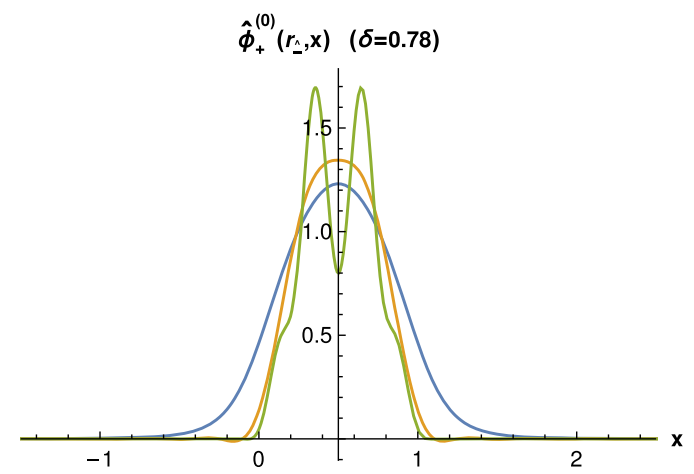

(e)

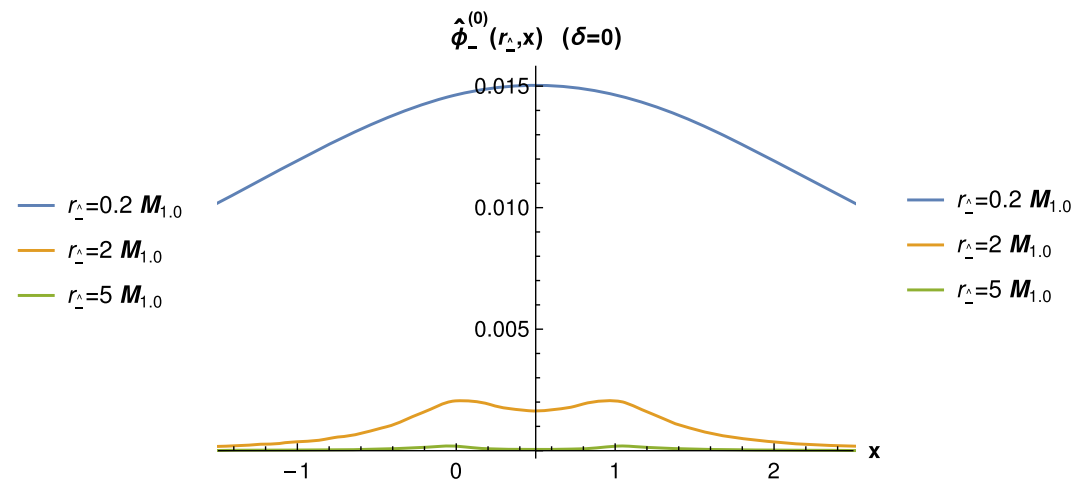

(b)

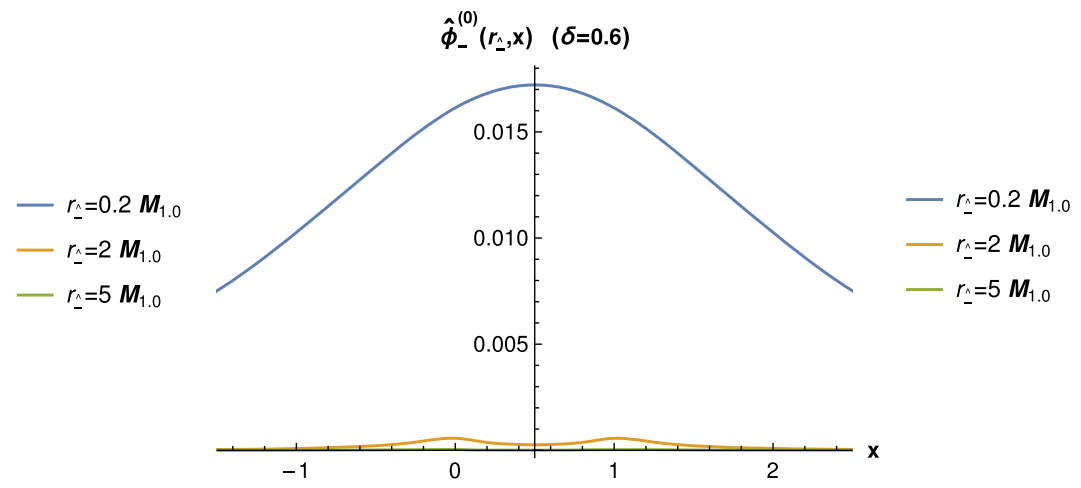

(d)
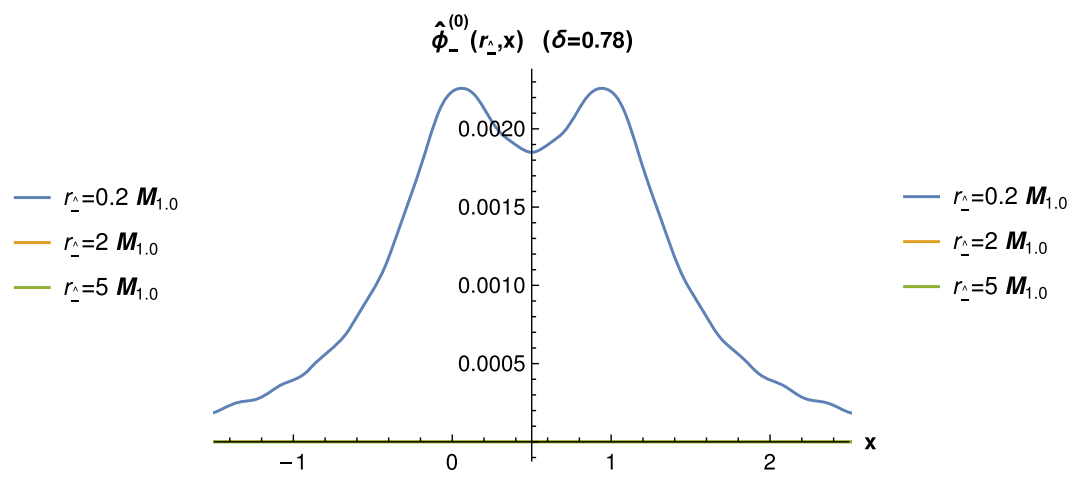

(f)

FIG. 31. Ground state wave functions $\hat{\phi}_{+}^{(0)}\left(r_{\hat{\iota}}, x\right)$ and $\hat{\phi}_{-}^{(0)}\left(r_{\hat{\iota}}, x\right)$ for $m=1.0$. All quantities are in proper units of $\sqrt{2 \lambda}$.

$\mathbf{M}_{1.0}=2.70$ (see Table VI) is the ground state meson mass for the quark mass value $\bar{m}=1.0$. The essential features that we discussed for the lower masses $(\bar{m}=0,0.045,0.18)$ in Sec. VIB and above remain without much change. As mentioned earlier, we have not increased the number of grid points beyond 600 while the numerical accuracy is much more demanded as $\mathbb{C}$ gets close to zero and $r_{\text {^ }}$ gets large. However, we are not alarmed by the appearance of "rabbit ear" for $\delta=0.78$ and $r_{\hat{\imath}}=5 \mathbf{M}_{1.0}=13.5$ in Fig. 31(e) as we are convinced from our numerical analyses that such numerical noise would disappear as we keep pushing the number of grid points even higher.

Finally, in Figs. 33 and 34, we present our numerical results of $\hat{\phi}_{+}^{(0)}\left(r_{\hat{\iota}}, x\right), \hat{\phi}_{-}^{(0)}\left(r_{\hat{\sim}}, x\right), \hat{\phi}_{+}^{(1)}\left(r_{\lrcorner}, x\right)$ and $\hat{\phi}_{-}^{(1)}\left(r_{\hat{\iota}}, x\right)$ for the case of $\bar{m}=2.11$. In this case, the $\theta\left(\bar{p}_{\hat{A}}^{\prime}\right)$ solution gets close to the free mass gap solution $\theta_{f}\left(\bar{p}_{\hat{\prime}}^{\prime}\right)$ as shown in Fig. 28, which may indicate that the binding effect gets lesser while the quark mass effect gets larger. In fact, the extreme heavy quark mass limit would yield the nonrelativistic $\delta$-function type of ground-state meson 


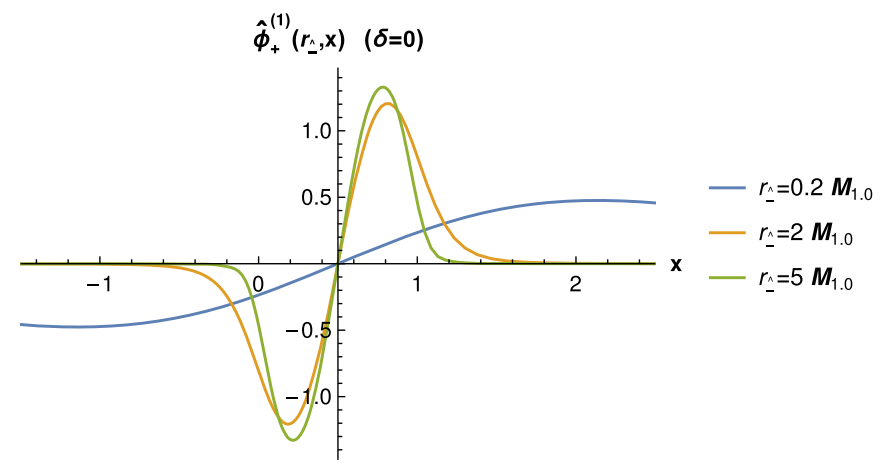

(a)

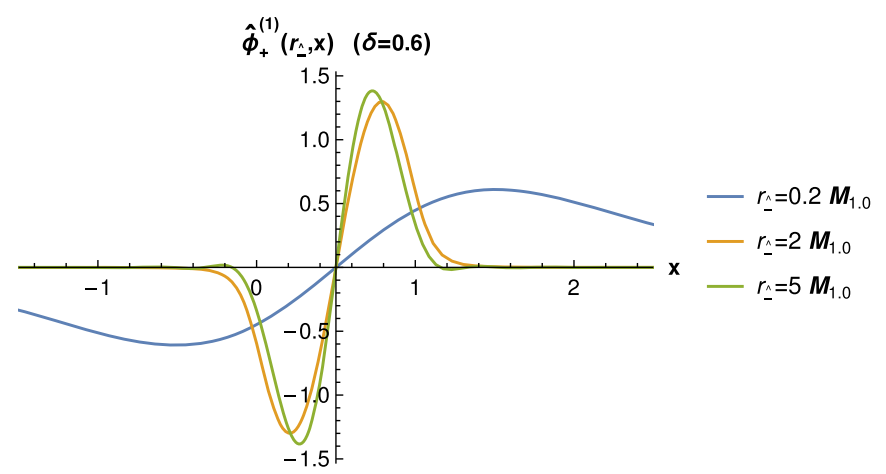

(c)

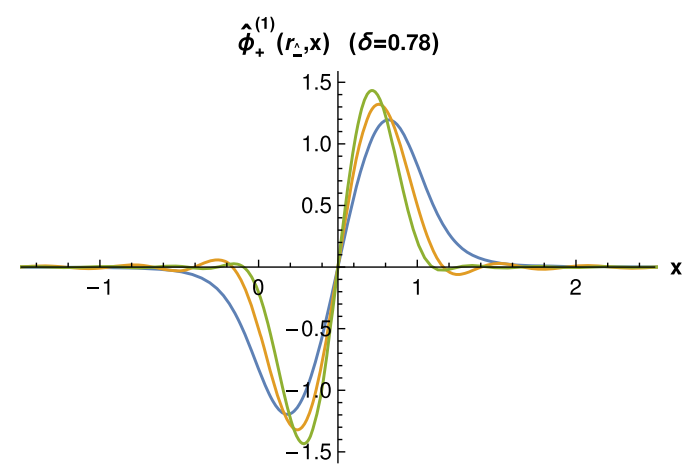

(e)

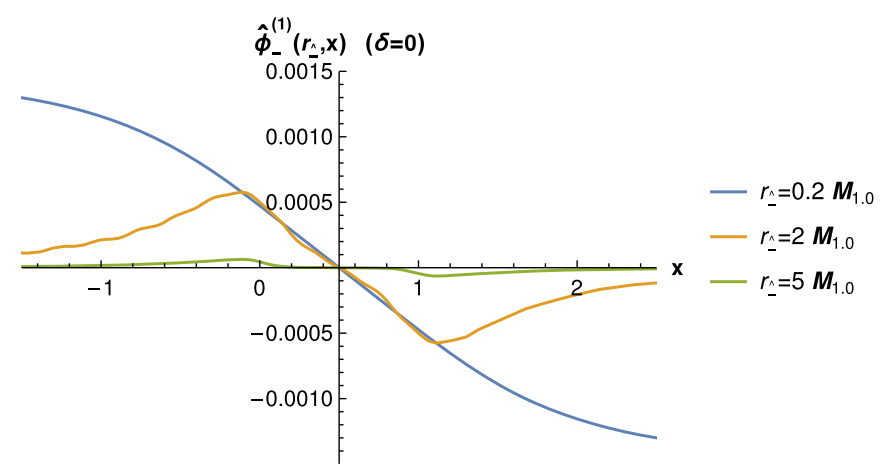

(b)

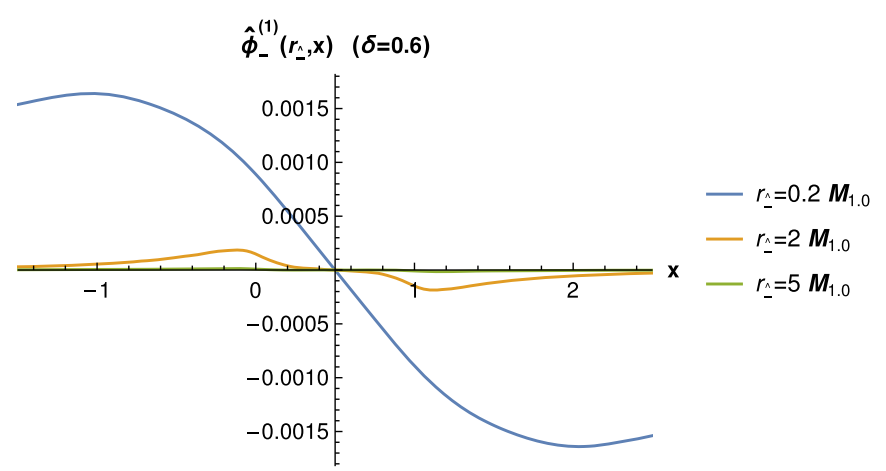

(d)

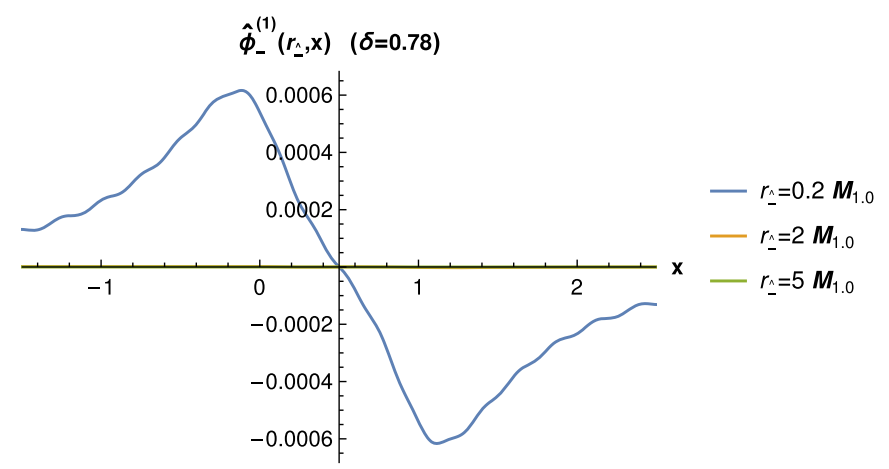

(f)

FIG. 32. First excited state wave functions $\hat{\phi}_{+}^{(1)}\left(r_{\lrcorner}, x\right)$ and $\hat{\phi}_{-}^{(1)}\left(r_{\lrcorner}, x\right)$ for $m=1$.0. All quantities are in proper units of $\sqrt{2 \lambda}$.

wave function peaked highly at $x=1 / 2$ to share the longitudinal momentum equally between the two equal mass quark and antiquark. In Fig. 33, we see a kind of precursor for such tendency toward the heavy quarkantiquark bound-state system. In the case of $\bar{m}=2.11$, we take our frames as $r_{\hat{\imath}}=0.2 \mathbf{M}_{2.11}, \mathbf{M}_{2.11}$ and $2 \mathbf{M}_{2.11}$, where $\mathbf{M}_{2.11}=4.91$ (see Table VI). As $2 \mathbf{M}_{2.11}$ is already large enough for our numerical computation, we do not go beyond $r_{\hat{\imath}}=2 \mathbf{M}_{2.11}$. Besides the tendency toward the heavy quark-antiquark bound-state system, the essential features that we discussed previously including the charge conjugation symmetry under the exchange of $x \leftrightarrow 1-x$ for the ground state and the first excited state appear similar in Figs. 33 and 34. We notice some wiggles in the $\hat{\phi}_{-}$component of the wave function solution in e.g., Fig. 33(f), but the overall magnitude of the $\hat{\phi}_{-}$wave function is always negligible compared to $\hat{\phi}_{+}$whenever this occurs, thus it does not cause concern to us. 


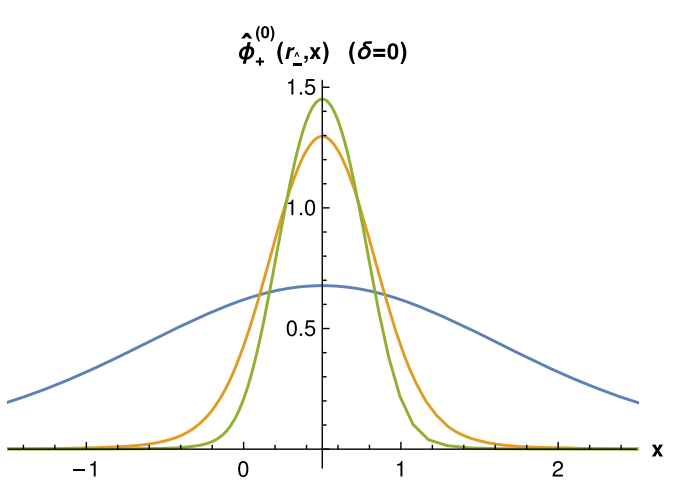

(a)

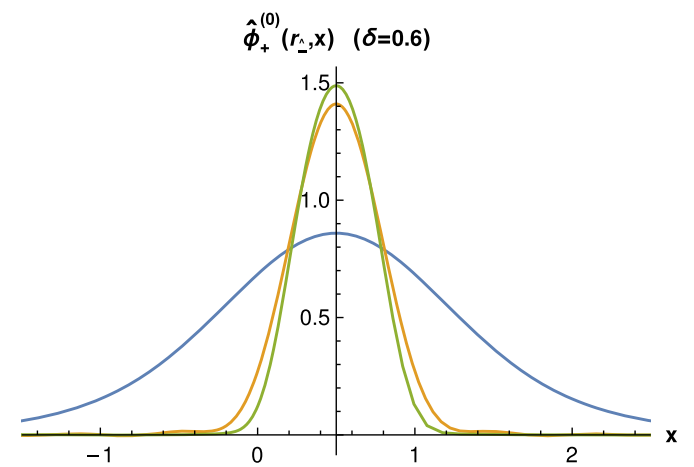

(c)

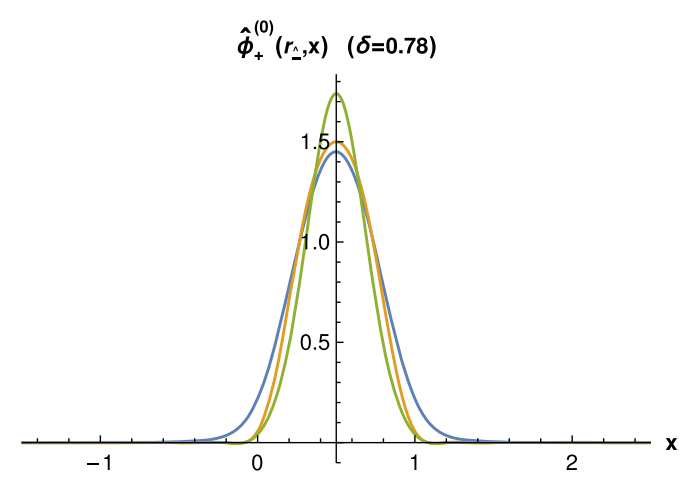

(e)

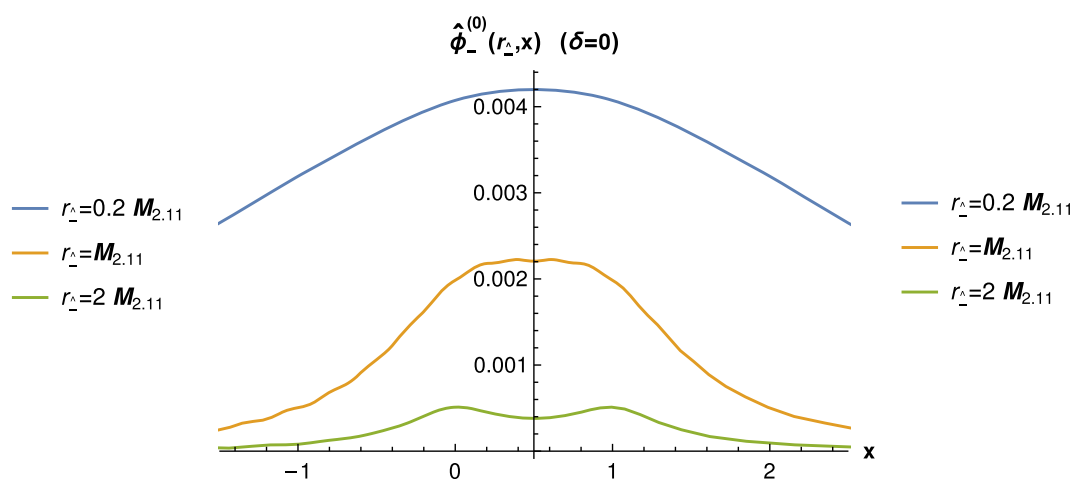

(b)
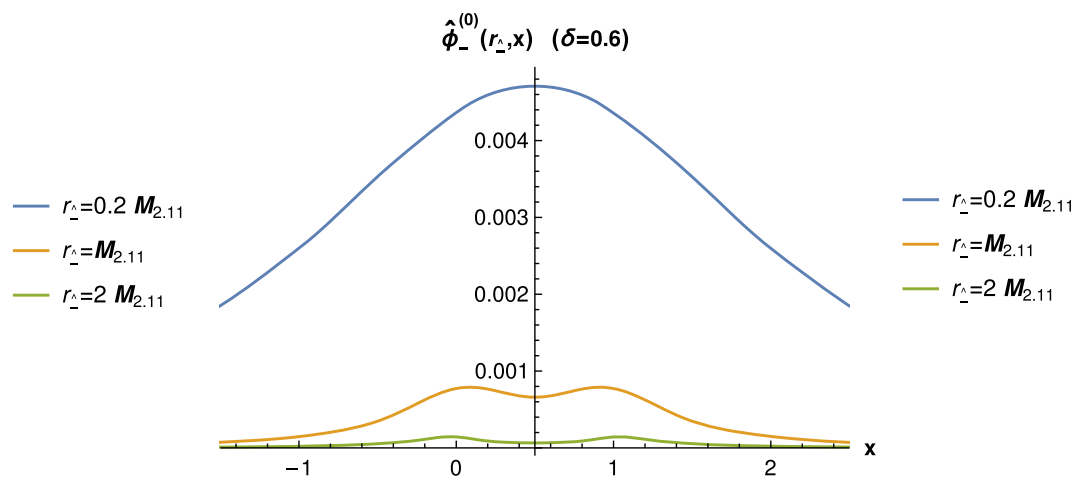

(d)
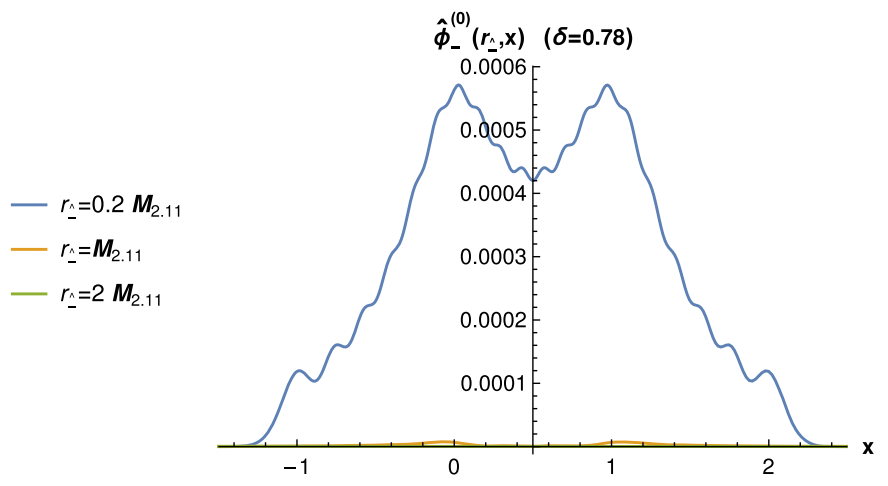

(f)

FIG. 33. Ground state wave functions $\hat{\phi}_{+}^{(0)}\left(r_{\lrcorner}, x\right)$ and $\hat{\phi}_{-}^{(0)}\left(r_{\underline{\Lambda}}, x\right)$ for $m=2.11$. All quantities are in proper units of $\sqrt{2 \lambda}$. 


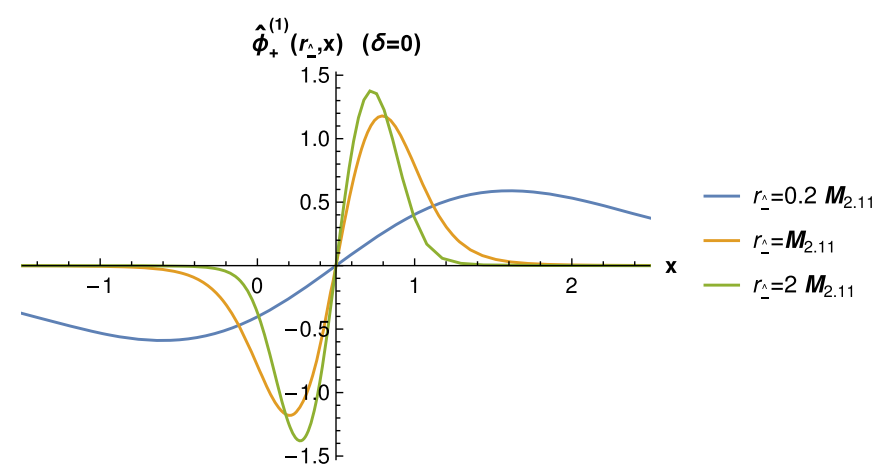

(a)

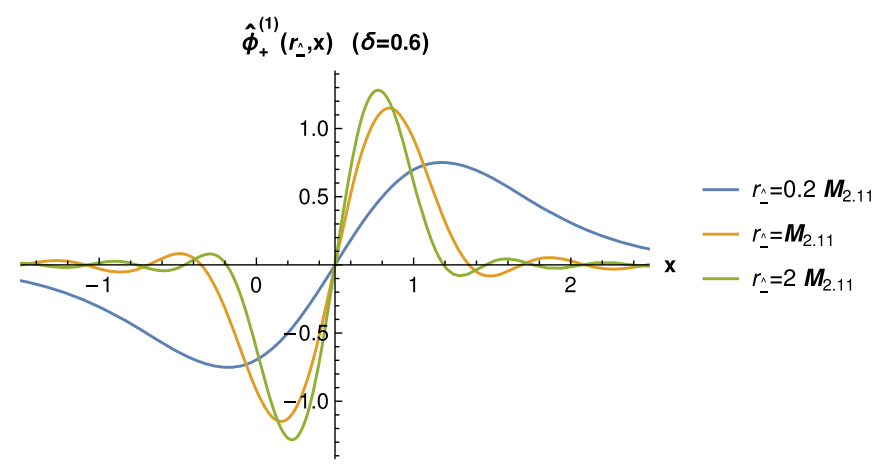

(c)

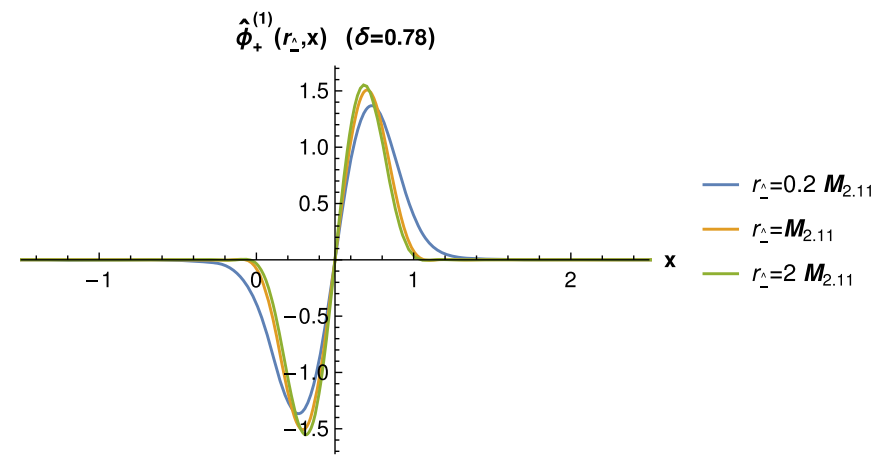

(e)

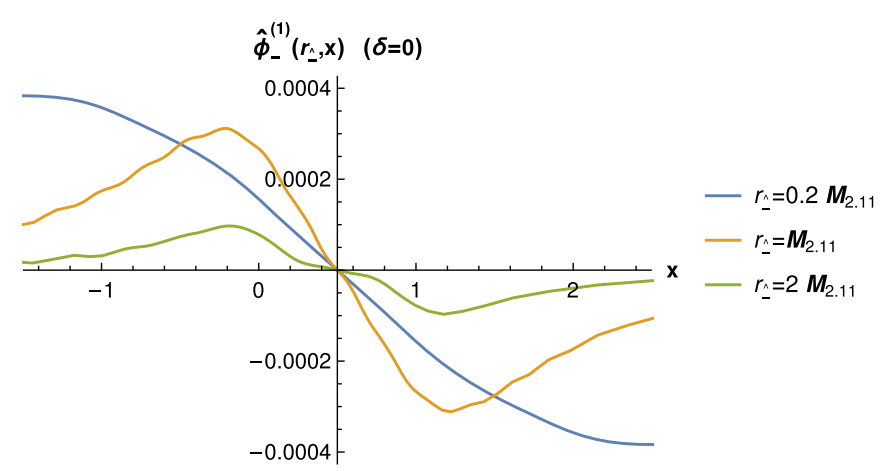

(b)

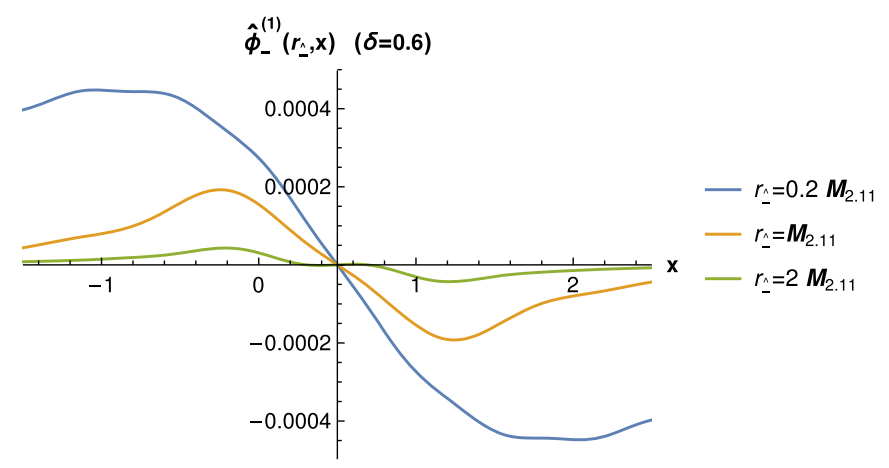

(d)

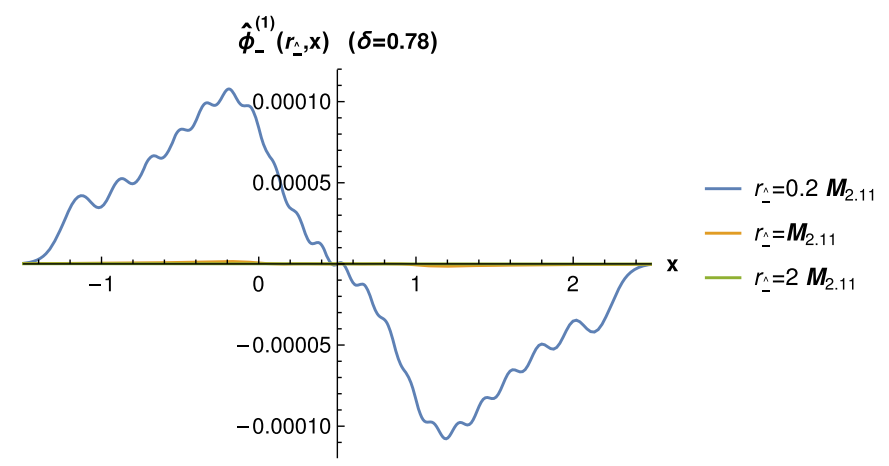

(f)

FIG. 34. First excited state wave functions $\hat{\phi}_{+}^{(1)}\left(r_{\hat{\iota}}, x\right)$ and $\hat{\phi}_{-}^{(1)}\left(r_{\hat{\iota}}, x\right)$ for $m=2.11$. All quantities are in proper units of $\sqrt{2 \lambda}$.

\section{APPENDIX E: "QUASI-PDFs" CORRESPONDING TO MESONIC WAVE FUNCTIONS FOR $\boldsymbol{m}=0.045$, 1.0, AND 2.11 IN THE UNIT OF $\sqrt{2 \lambda}$}

Starting from the definition of the "quasi-PDFs" interpolating between IFD and LFD given by Eq. (129), we obtained the "quasi-PDFs" using the mesonic wave functions of the quark-antiquark bound states in the interpolating axial gauge, $A_{\wedge}^{a}=0$, as given by Eq. (132) and discussed the "quasi-PDFs" for the cases of $m=0$ and $m=0.18$ in Sec. VIC. As the mesonic wave functions for $m=0.045,1.0$, and 2.11 were presented in the previous Appendix D, we now discuss the corresponding "quasiPDFs" in this Appendix E.
First, the $\delta=0$ (IFD) results of the ground state and first excited state mesonic quasi-PDFs are shown in Figs. 35 and 36 , respectively, for a few different quark mass values, not only $m=0.045$ as taken in Ref. [22] but also $m=1.0$ and $m=2.11$. The results of $m=0.045$ shown in Figs. 35(a) and 36(a) agree very well with the top right panels of Figs. 2 and 3 of Ref. [22]. Due to the charge conjugation symmetry under the exchange of $x \leftrightarrow 1-x, \hat{\phi}_{ \pm}^{(0)}\left(r_{\lrcorner}, x\right)=$ $\hat{\phi}_{ \pm}^{(0)}\left(r_{\hat{\sim}}, 1-x\right)$ and $\hat{\phi}_{ \pm}^{(1)}\left(r_{\hat{\varkappa}}, x\right)=-\hat{\phi}_{ \pm}^{(1)}\left(r_{\hat{\iota}}, 1-x\right)$, we see the peak and valley at $x=1 / 2$ for $\tilde{q}_{0}\left(r_{\hat{\Delta}}, x\right)$ and $\tilde{q}_{1}\left(r_{\hat{\Delta}}, x\right)$, respectively, as shown in Figs. 35 and 36. Although the peak and valley get a little more sharpened as $m$ gets larger, the essential features of the symmetry remain intact 


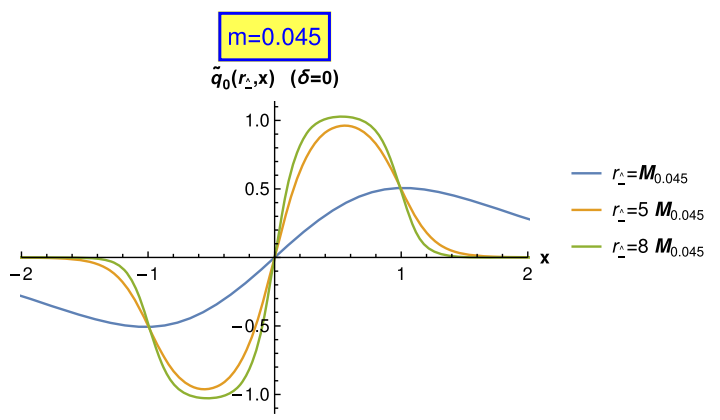

(a)

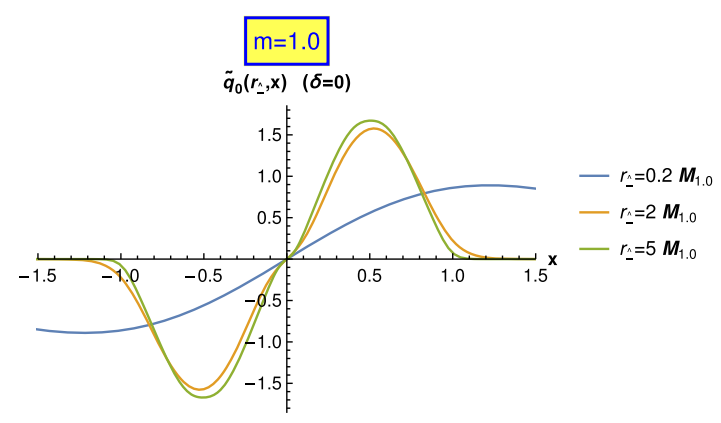

(b)

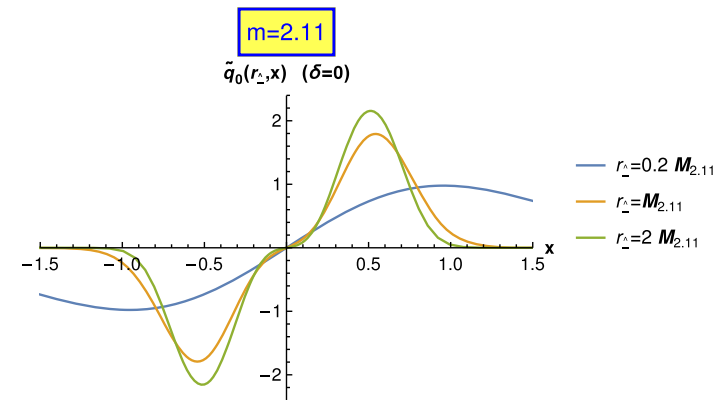

(c)

FIG. 35. Quasi-PDFs in IFD $(\delta=0)$ for the ground state $(n=0)$ wave functions of (a) $m=0.045$, (b) $m=1.00$, and (c) $m=2.11$ in the unit of $\sqrt{2 \lambda}$.

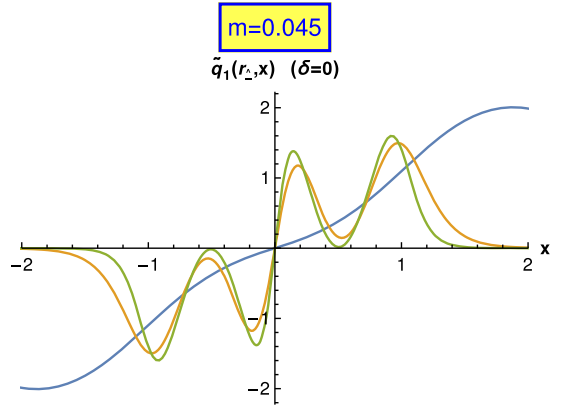

(a)

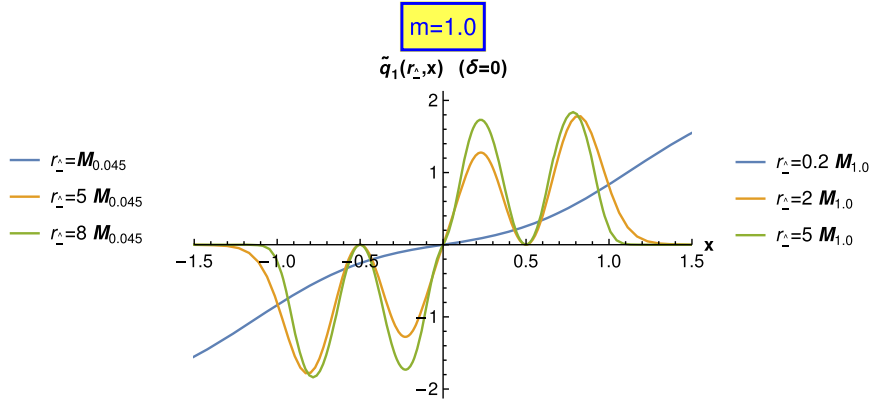

(b)

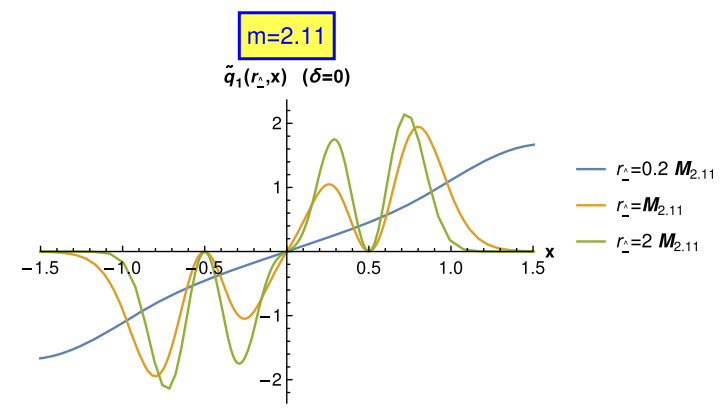

(c)

FIG. 36. Quasi-PDFs in IFD $(\delta=0)$ for the first excited state $(n=1)$ wave functions of (a) $m=0.045$, (b) $m=1.00$, and (c) $m=2.11$ in the unit of $\sqrt{2 \lambda}$. 


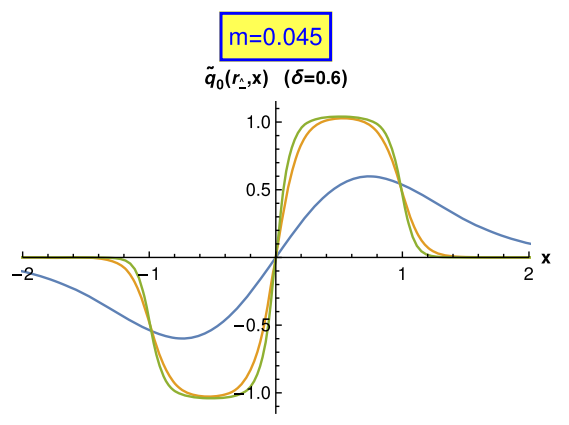

(a)

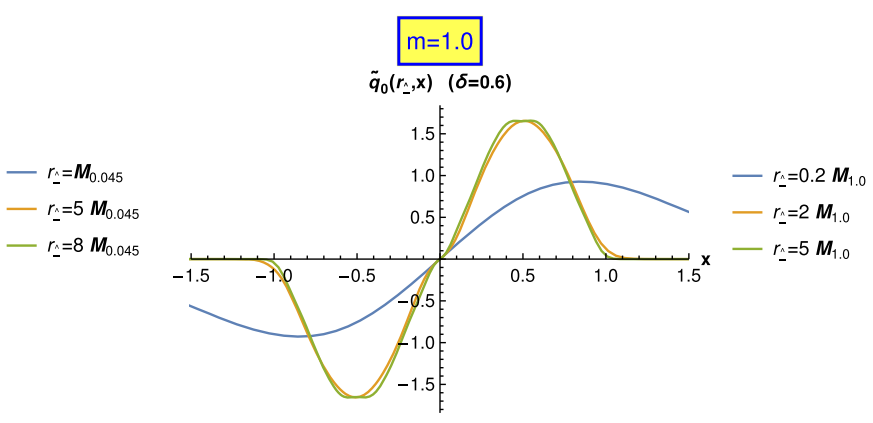

(b)

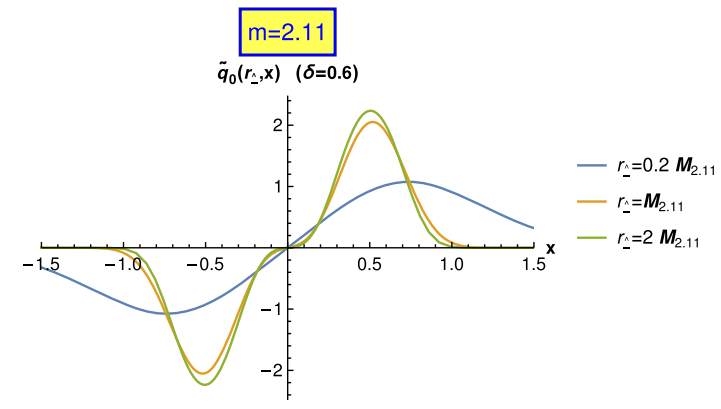

(c)

FIG. 37. $\delta=0.6$ interpolating "quasi-PDFs" for the ground state $(n=0)$ wave functions of (a) $m=0.045$, (b) $m=1.00$, and (c) $m=2.11$ in the unit of $\sqrt{2 \lambda}$.

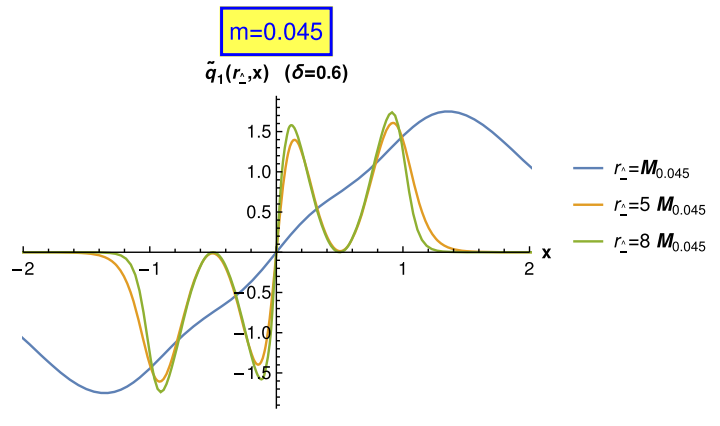

(a)

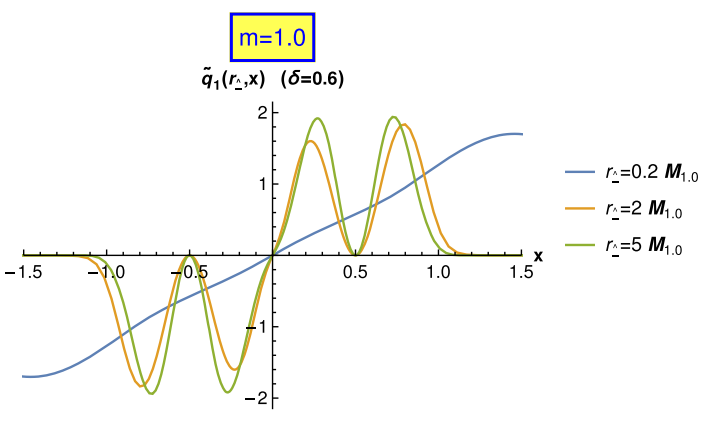

(b)

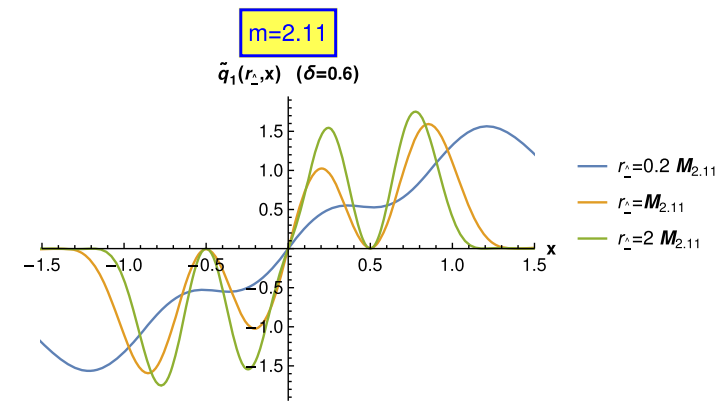

(c)

FIG. 38. $\delta=0.6$ interpolating "quasi-PDFs" for the first excited state $(n=1)$ wave functions of (a) $m=0.045$, (b) $m=1.00$, and (c) $m=2.11$ in the unit of $\sqrt{2 \lambda}$. 


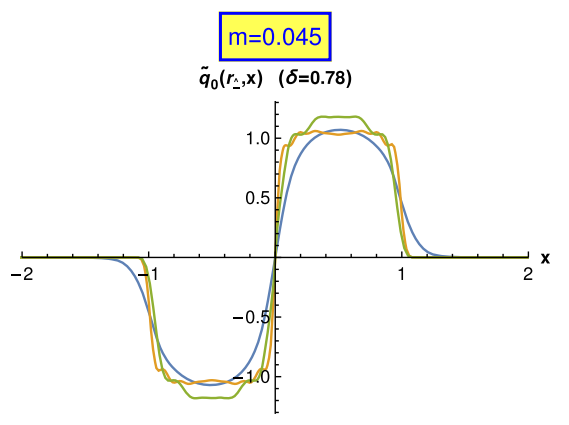

(a)

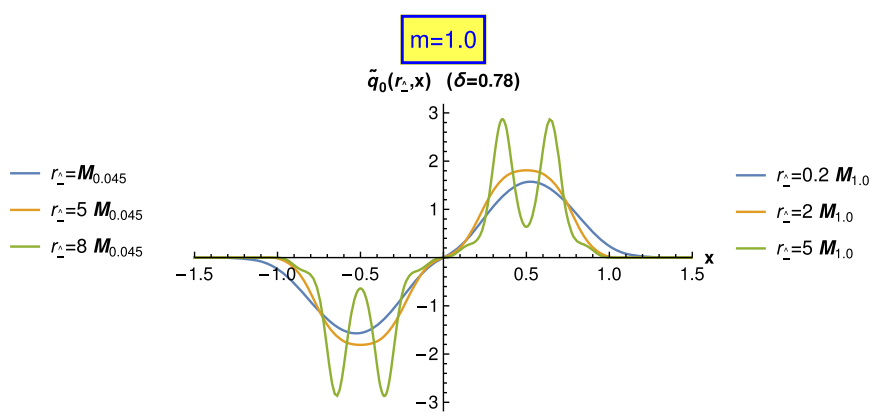

(b)

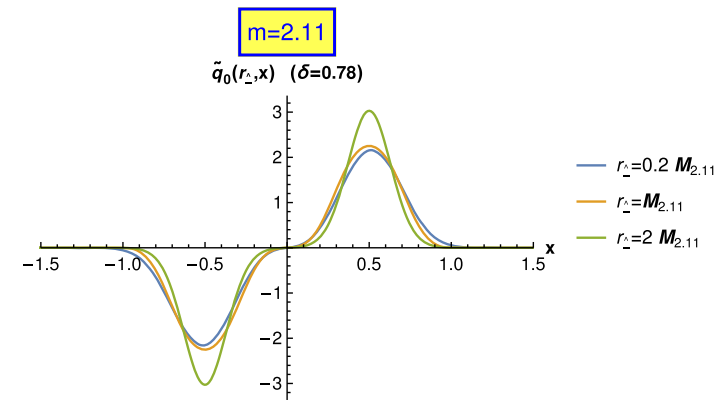

(c)

FIG. 39. $\delta=0.78$ interpolating "quasi-PDFs" for the ground state $(n=0)$ wave functions of (a) $m=0.045$, (b) $m=1.00$, and (c) $m=2.11$ in the unit of $\sqrt{2 \lambda}$.

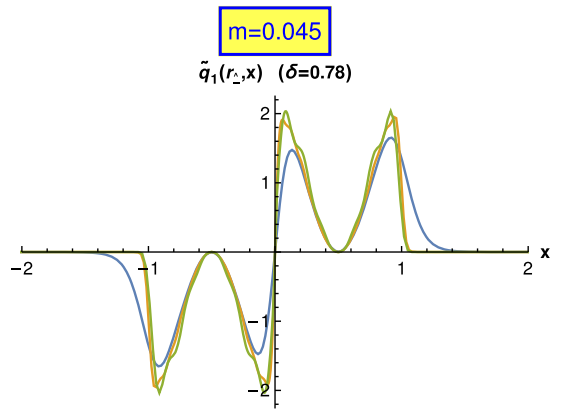

(a)

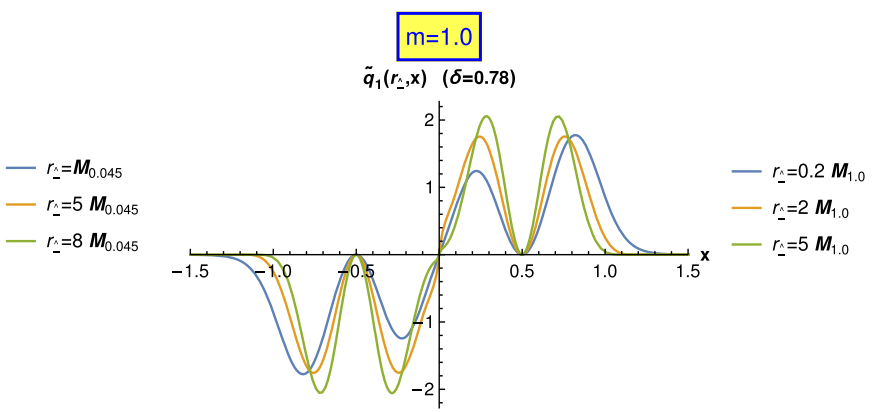

(b)

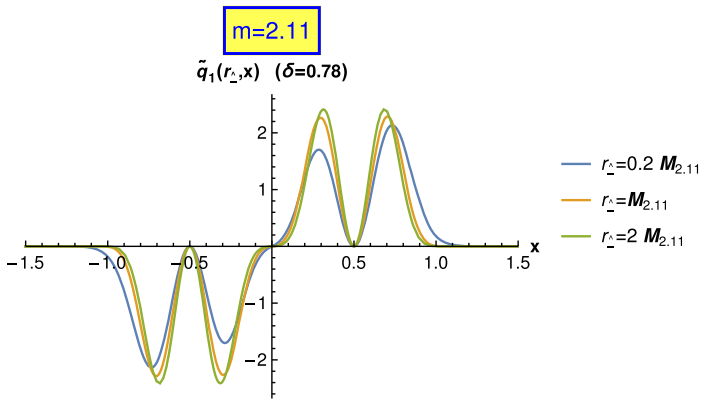

(c)

FIG. 40. $\delta=0.78$ interpolating "quasi-PDFs" for the first excited state $(n=1)$ wave functions of (a) $m=0.045$, (b) $m=1.00$, and (c) $m=2.11$ in the unit of $\sqrt{2 \lambda}$. 
regardless of the $m$ values. In each panel, the results of the moving frames with the longitudinal meson momentum $r_{\hat{\iota}}=r^{1}=\mathbf{M}_{0.045}, 5 \mathbf{M}_{0.045}$, and $8 \mathbf{M}_{0.045}$, where $\mathbf{M}_{0.045}=$ 0.42 (see Table VI) is the ground state meson mass for the quark mass value $m=0.045$, are depicted by the solid lines in blue, yellow, and green, respectively. As noted in Ref. [22], the large-momentum IFD $(\delta=0)$ numerical results approach to the LFD results quite slowly as the momentum $r_{\hat{\imath}}=r^{1}$ gets large. The corresponding results for the larger $m$ values, $m=1.0$ and $m=2.11$, are shown in Figs. 35(b) and 36(b) and Figs. 35(c) and 36(c) for the ground state and the first excited state, respectively.

As discussed in Sec. VIC, the variation of the interpolating parameter $\delta$ may remedy the slow approach to the LFD results in IFD $(\delta=0)$. To exhibit this feature, we show the results of the ground state and first excited state mesonic quasi-PDFs for different $\delta$ values $(\delta=0.6$ and 0.78) in Figs. 37-40, respectively, with the same arrangement of corresponding $m$ values ( $m=0.045,1.0$ and 2.11). For the $\delta=0.6$ case shown in Figs. 37 and 38, one may see already some improvement in the approach to the LFD results by comparing the corresponding $\tilde{q}_{0}\left(r_{\hat{\wedge}}, x\right)$ and $\tilde{q}_{1}\left(r_{\wedge}, x\right)$ results in Figs. 35 and 36 with the corresponding $r_{\wedge}$ values. For the $\delta=0.78$ case shown in Figs. 39 and 40, the results get improved much more dramatically yielding very quickly the essential features of the LFD results fitting in the region $x \in[0,1]$. The similar features have been noted earlier in Secs. VIB and VIC as well as in Appendix D, i.e., taking $\delta$ away from the IFD $(\delta=0)$, the resemblance to the PDFs in LFD may appear more swiftly achieved with the boost of the meson longitudinal momentum $r_{\wedge}$ to the larger value. Nevertheless, one should note here a numerical caveat demanding much higher numerical accuracy as the meson momentum $r_{-}$gets larger while the $\delta$ value gets close to $\pi / 4$ (e.g., $\delta=0.78$ ). In such situation, the numerical sensitivity kicks in so strongly that the results cannot be trusted unless they get tested for the improvement with much higher numerical accuracy. However, as discussed in Sec. VIC, one does not need to boost the longitudinal momentum $r_{\wedge}$ too large if the $\delta$ value gets close to $\pi / 4$. As the $\delta$ value gets close to $\pi / 4$, relatively smaller $r_{\hat{\imath}}$ value can do the job. Thus, it would be worthwhile to search for a "sweet spot" by varying both $\delta$ and $r_{\text {^ }}$ together to obtain the "LFD-like" result.

\section{APPENDIX F: REST FRAME BOUND-STATE EQUATION AND ITS SOLUTION}

While we presented our numerical solutions of the bound-state wave functions $\hat{\phi}_{ \pm}^{(n)}\left(r_{\hat{\iota}}, x\right)$ in terms of the interpolating longitudinal momentum fraction variable $x=$ $p_{\iota} / r_{\iota}$ in Sec. VI B and Appendix D to discuss the moving frame dependence of the interpolating wave functions between IFD and LFD, the rest frame is special and deserves separate description/discussion. In particular, the massless particles cannot exist in the rest frame according to the relativity although the GOR relation $\mathcal{M}_{(0)}^{2} \sim m \sqrt{\lambda} \rightarrow 0$ indicates that the meson mass $\mathcal{M}_{(0)} \rightarrow 0$ as $m \rightarrow 0$ in the chiral limit. As the massless Goldstone boson moves with the speed of light, it cannot exist in the rest frame. We thus devote this final Appendix for the discussion of the rest frame bound-state equation and its solution.

Taking $r_{\hat{\imath}}=0$ in Eq. (116), we get

$$
\begin{aligned}
& {\left[-r^{\hat{+}}+2 E\left(p_{\lrcorner}\right)\right] \hat{\phi}_{+}\left(p_{\hat{\iota}}\right)} \\
& =\lambda \mathbb{C} f \frac{d k_{\hat{\varkappa}}}{\left(p_{\hat{\imath}}-k_{\hat{\varkappa}}\right)^{2}}
\end{aligned}
$$

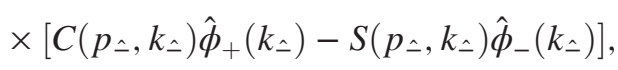

$$
\begin{aligned}
& {\left[r^{\hat{+}}+2 E\left(p_{\hat{\varkappa}}\right)\right] \hat{\phi}_{-}\left(p_{\hat{\lrcorner}}\right)} \\
& =\lambda \mathbb{C} f \frac{d k_{\hat{-}}}{\left(p_{\hat{\imath}}-k_{\hat{\iota}}\right)^{2}}
\end{aligned}
$$

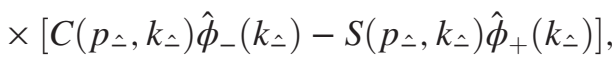

where

$C\left(p_{\hat{\lrcorner}}, k_{\hat{\varkappa}}\right)=C\left(p_{\hat{\lrcorner}}, k_{\hat{\iota}}, r_{\hat{\iota}}=0\right)=\cos ^{2}\left(\frac{\theta\left(p_{\hat{\varkappa}}\right)-\theta\left(k_{\hat{\lrcorner}}\right)}{2}\right)$,

and

$S\left(p_{\hat{\iota}}, k_{\hat{\iota}}\right)=S\left(p_{\hat{\iota}}, k_{\hat{\iota}}, r_{\hat{\iota}}=0\right)=-\sin ^{2}\left(\frac{\theta\left(p_{\hat{\iota}}\right)-\theta\left(k_{\hat{\iota}}\right)}{2}\right)$.

The basis wave function is also provided without scaling the interpolating momentum variable $p_{\wedge}$ with respect to $r_{\text {. }}$ [in contrast to Eq. (125)]

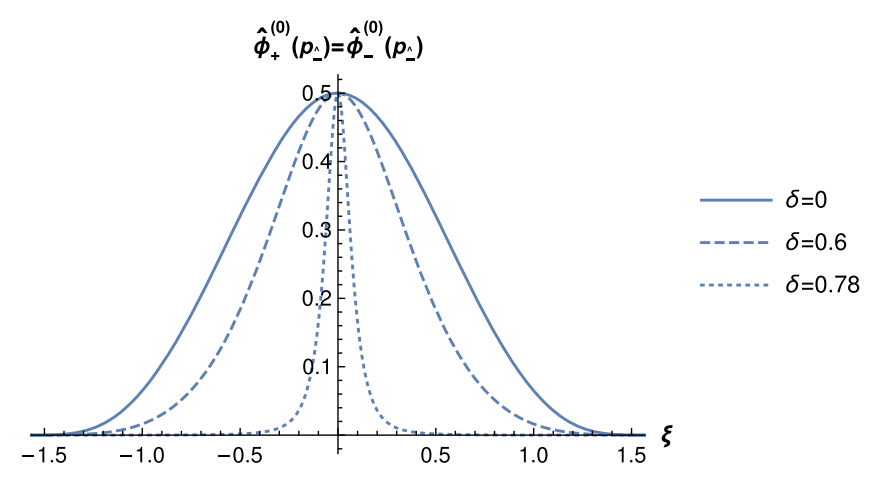

FIG. 41. Analytic solutions for the ground state wave functions in the rest frame for the bare quark mass $m=0$ for three different interpolation angles as functions of $\xi=\tan ^{-1} p_{\hat{\Lambda}}$. All quantities are in proper units of $\sqrt{2 \lambda}$. 


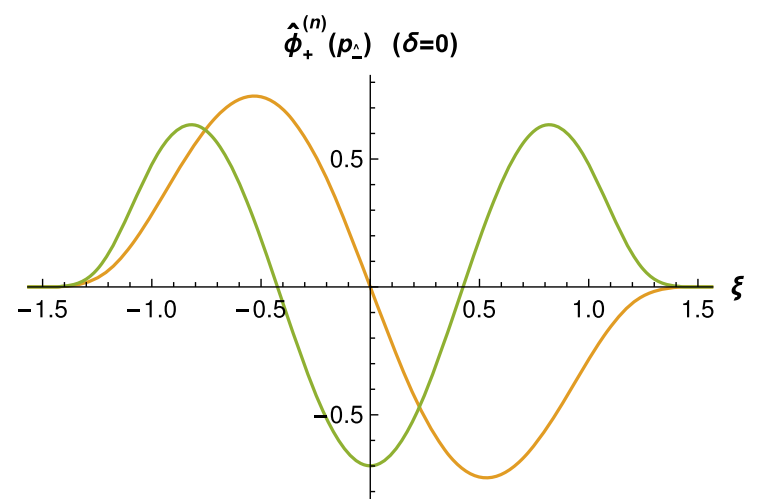

(a)

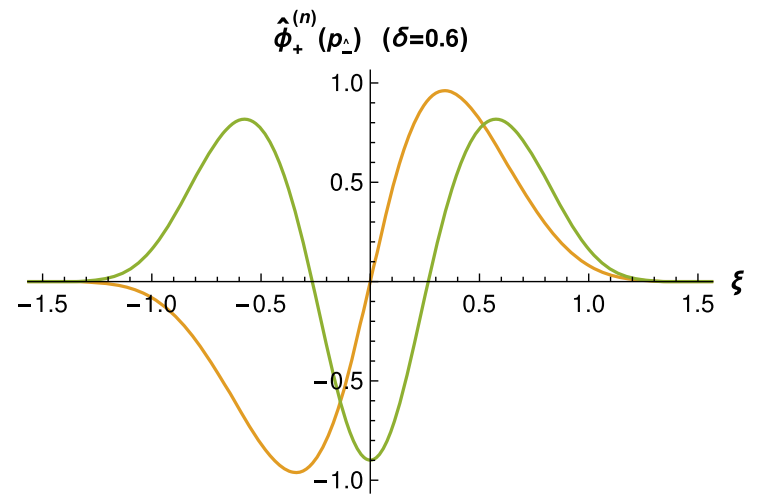

(c)

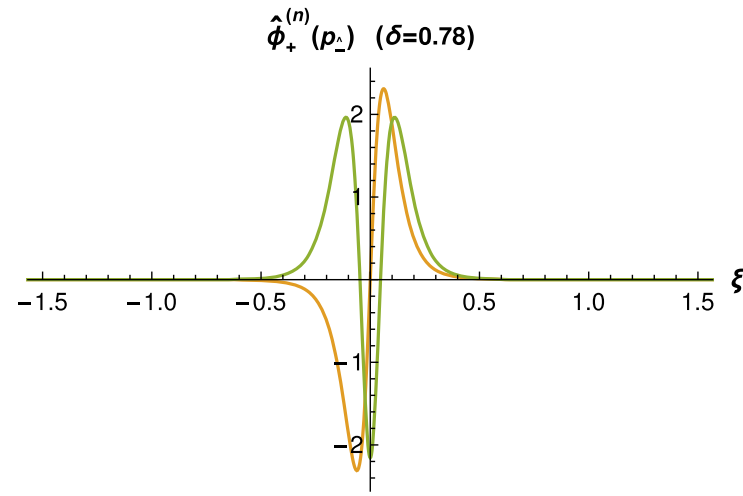

(e)

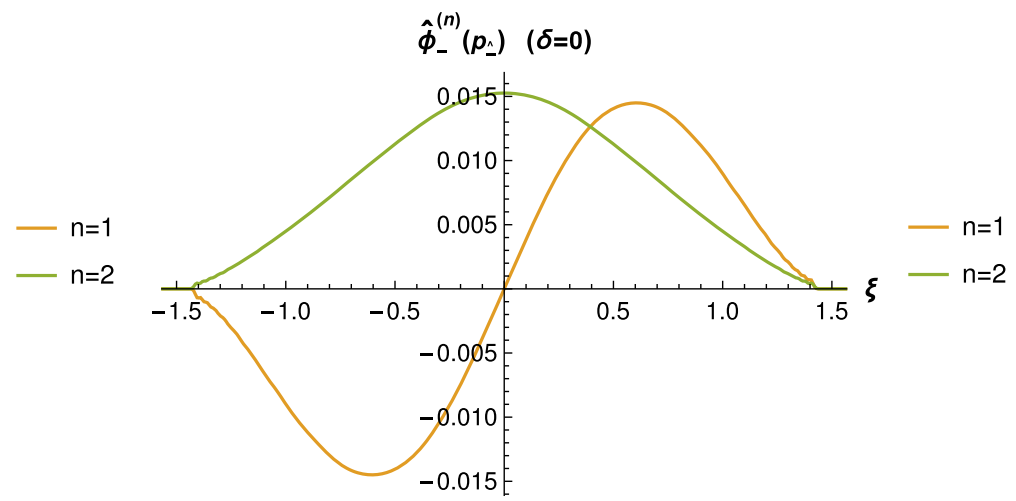

(b)

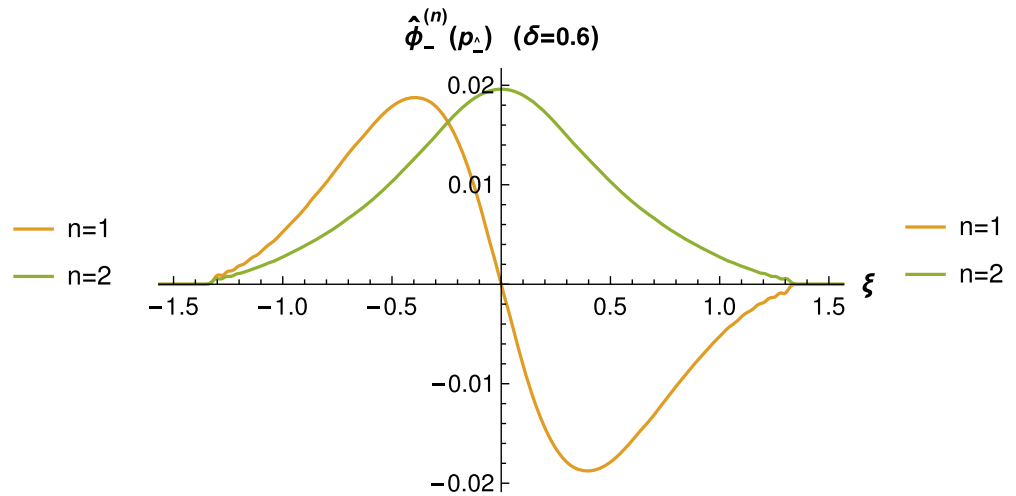

(d)

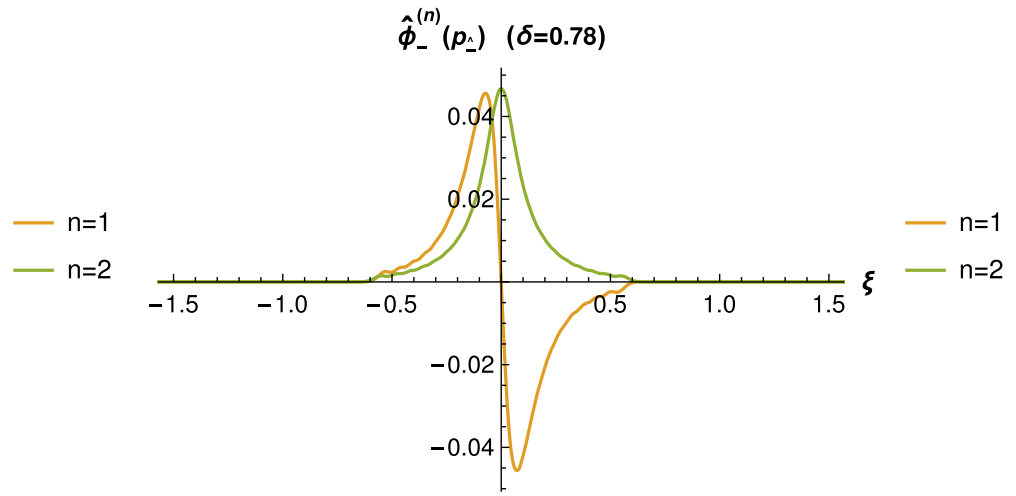

(f)

FIG. 42. Rest frame wave functions $\hat{\phi}_{+}^{(n)}\left(p_{\hat{\sim}}\right)$ and $\hat{\phi}_{-}^{(n)}\left(p_{\hat{-}}\right)$ for $m=0(n=1$ and $n=2)$ as functions of $\xi=\tan ^{-1} p_{\hat{\iota}}$. All quantities are in proper units of $\sqrt{2 \lambda}$.

$\Psi_{m}\left(\alpha, p_{\hat{\iota}}\right)=\sqrt{\frac{\alpha}{2^{m} m ! \sqrt{\pi}}} \exp \left[-\frac{1}{2} \alpha^{2} p_{\hat{\iota}}^{2}\right] H_{m}\left(\alpha p_{\hat{\iota}}\right)$,

and the $n$th bound-state wave functions $\hat{\phi}_{ \pm}^{(n)}\left(p_{\hat{\Delta}}\right)$ are normalized as

$$
\int d p_{\hat{\iota}}\left\{\left|\hat{\phi}_{+}^{(n)}\left(p_{\hat{\varkappa}}\right)\right|^{2}-\left|\hat{\phi}_{-}^{(n)}\left(p_{\hat{\varkappa}}\right)\right|^{2}\right\}=1,
$$

where one should note the caveat of $n=0$ solution for $m=0$ that becomes null in the rest frame due to the relativity.

In the frame $r_{\hat{\imath}}=0$, the meson mass square $\mathcal{M}_{(n)}^{2}=$ $\frac{\left(r_{(n)}^{\hat{+}}\right)^{2}}{\mathbb{C}}$ and the corresponding wave functions $\hat{\phi}_{ \pm}^{(n)}\left(p_{\hat{\varkappa}}\right)$ are obtained by solving the coupled bound-state equations, Eqs. (F1a) and (F1b), using essentially the same technique that we described in Sec. VI A.

Due to the GOR relation $\mathcal{M}_{(0)}^{2} \sim m \sqrt{\lambda} \rightarrow 0$, the groundstate meson mass $\mathcal{M}_{(0)} \rightarrow 0$ as $m \rightarrow 0$. As mentioned 


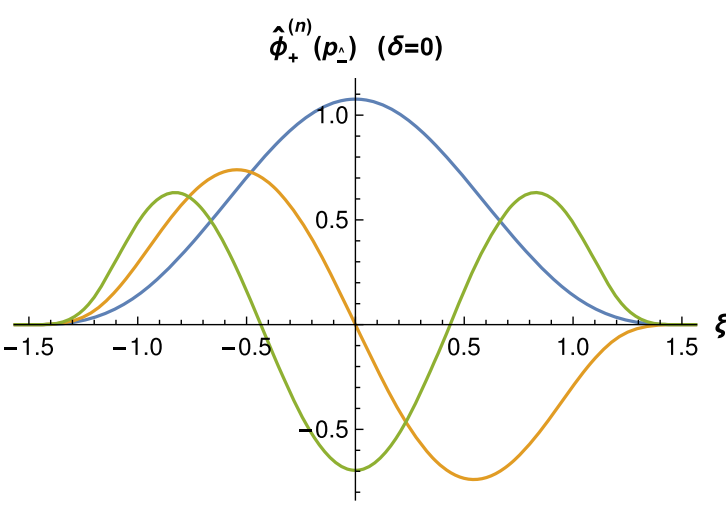

(a)
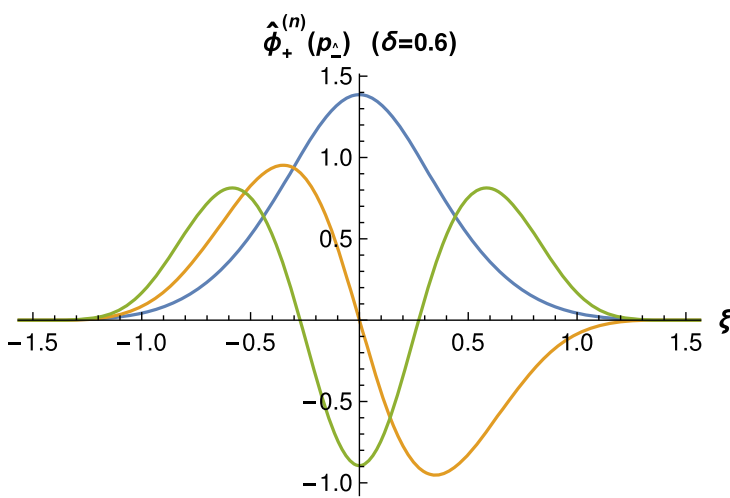

(c)

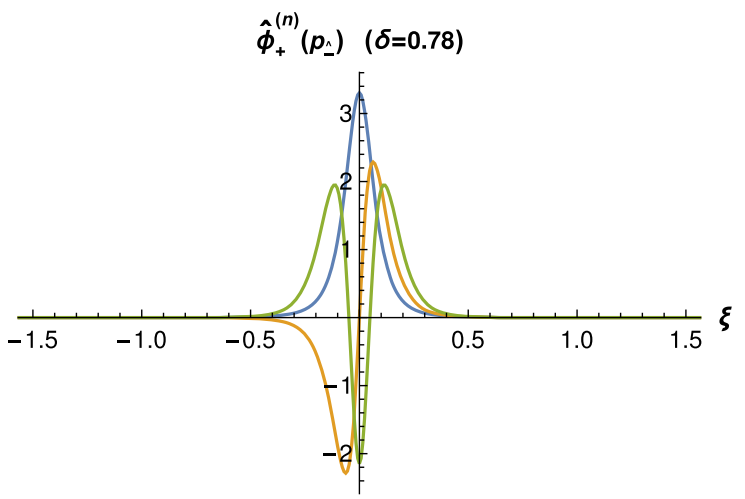

(e)

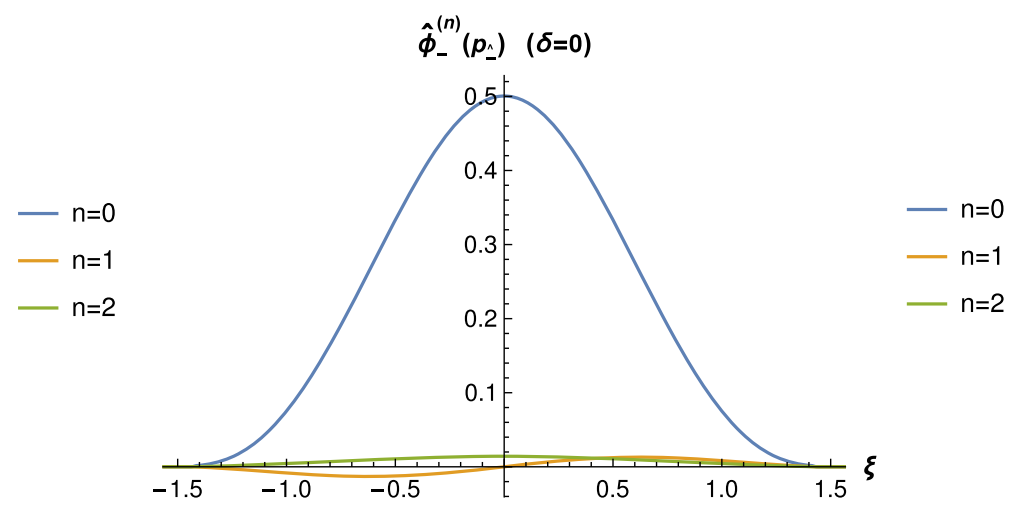

(b)

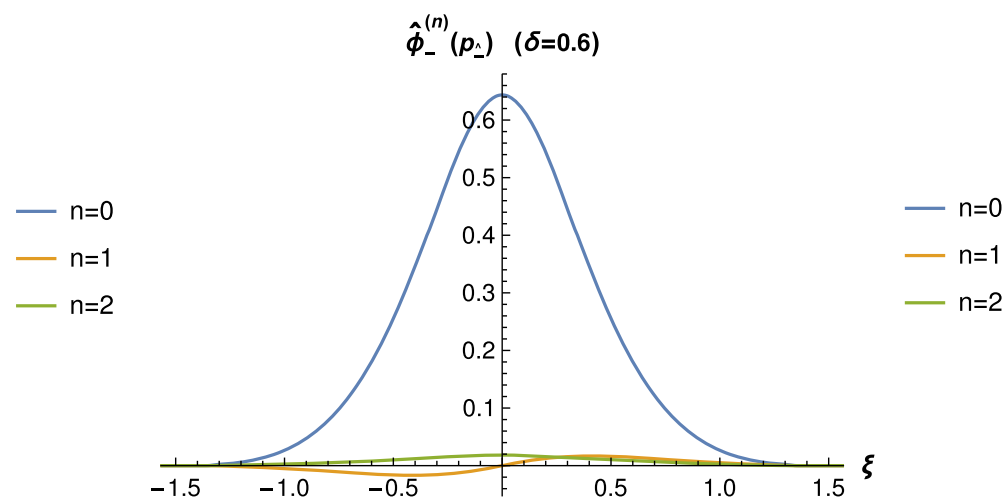

(d)
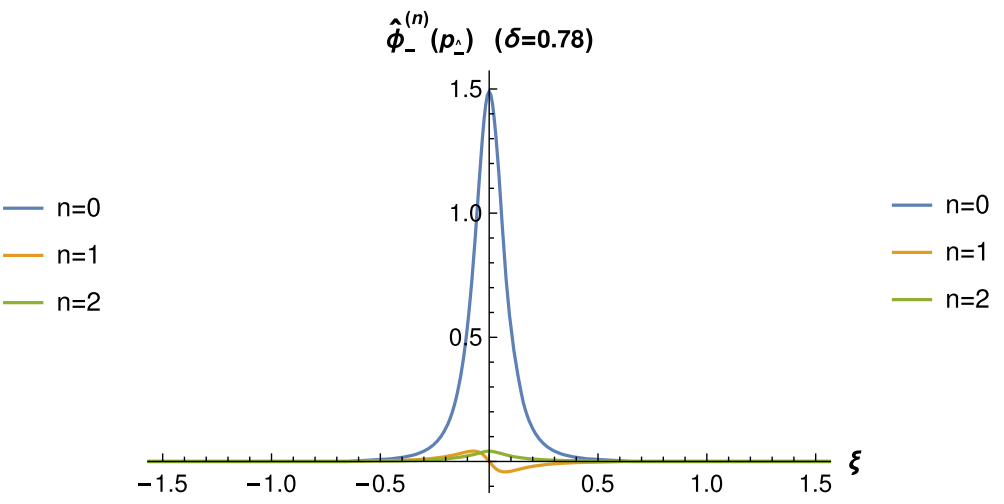

(f)

FIG. 43. Rest frame wave functions $\hat{\phi}_{+}^{(n)}\left(p_{\lrcorner}\right)$and $\hat{\phi}_{-}^{(n)}\left(p_{\lrcorner}\right)$for $m=0.045$ as functions of $\xi=\tan ^{-1} p_{\wedge}$. All quantities are in proper units of $\sqrt{2 \lambda}$.

earlier, the massless Goldstone boson then moves with the speed of light and cannot exist in the rest frame according to the relativity. How can one understand this distinction of the massless Goldstone boson in the rest frame? To realize it, one may take a look more closely the analytic solution of the ground-state $(n=0)$ wave function $\left(\hat{\phi}_{ \pm}^{(0)}\left(p_{\hat{\iota}}\right)\right)$ given by Eq. (128) and find that the rest frame $r_{\hat{\imath}}=0$ yields $\hat{\phi}_{ \pm}^{(0)}\left(p_{\wedge}\right)$ as

$$
\hat{\phi}_{+}^{(0)}\left(p_{\hat{\lrcorner}}\right)=\hat{\phi}_{-}^{(0)}\left(p_{\hat{\lrcorner}}\right)=\frac{1}{2} \cos \theta\left(p_{\hat{\lrcorner}}\right) .
$$

Then, indeed, the normalization condition given by Eq. (124) becomes null indicating the absence of the massless Goldstone boson in the rest frame. However, the individual interpolating wave functions $\hat{\phi}_{+}^{(0)}\left(p_{\hat{\sim}}\right)$ and $\hat{\phi}_{-}^{(0)}\left(p_{\hat{\iota}}\right)$ do not vanish as plotted in Fig. 41. Here, we use 


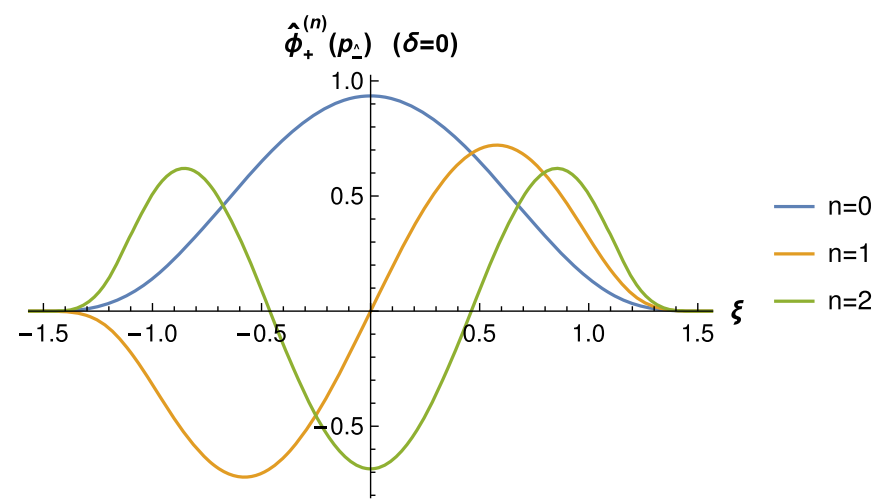

(a)

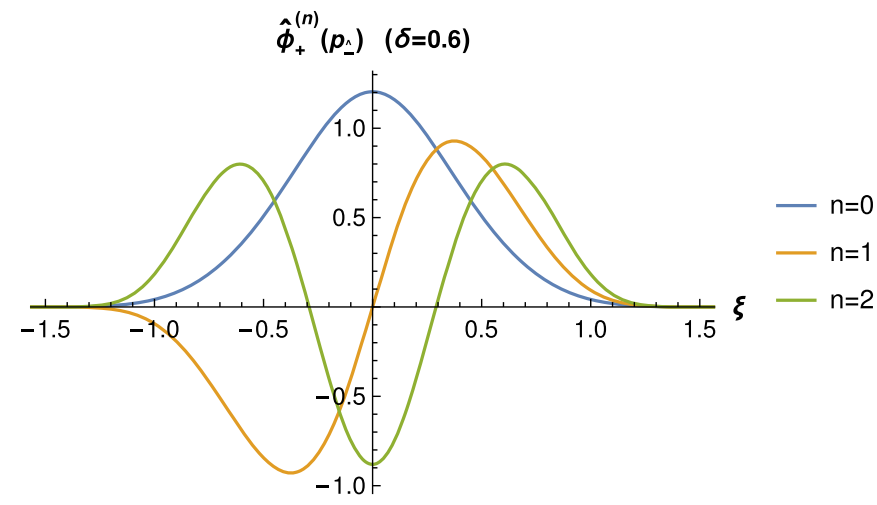

(c)

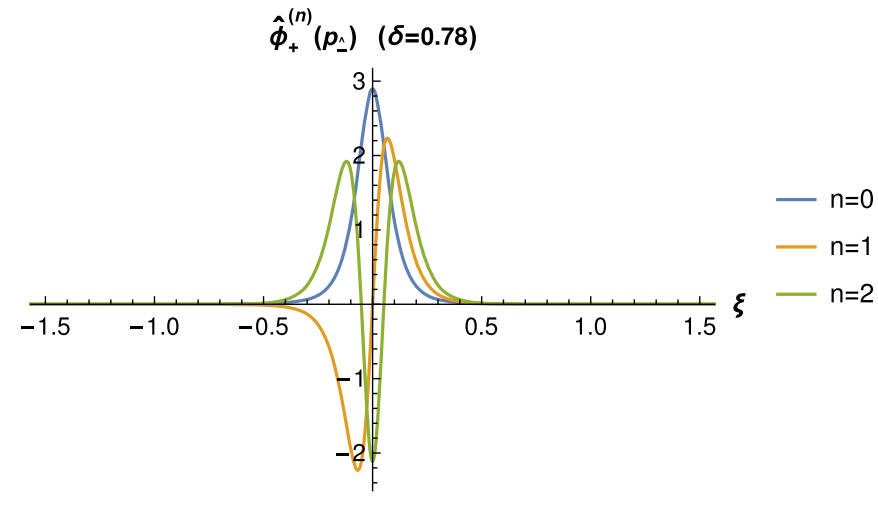

(e)

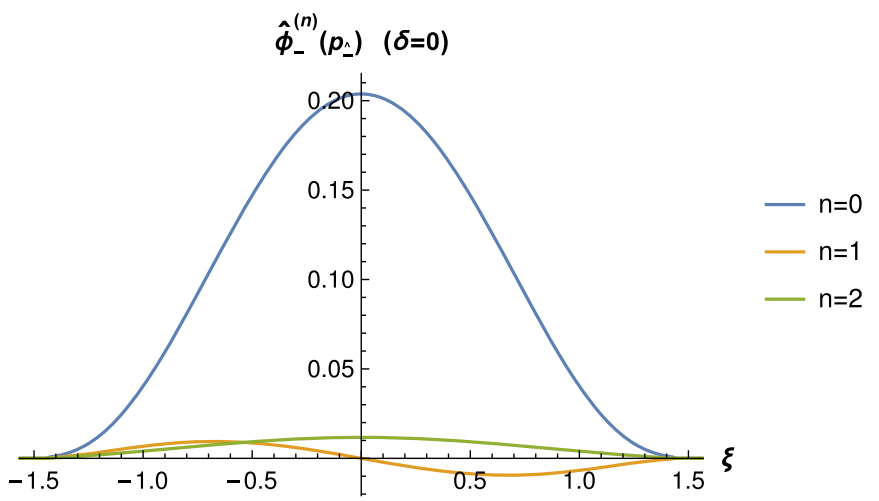

(b)

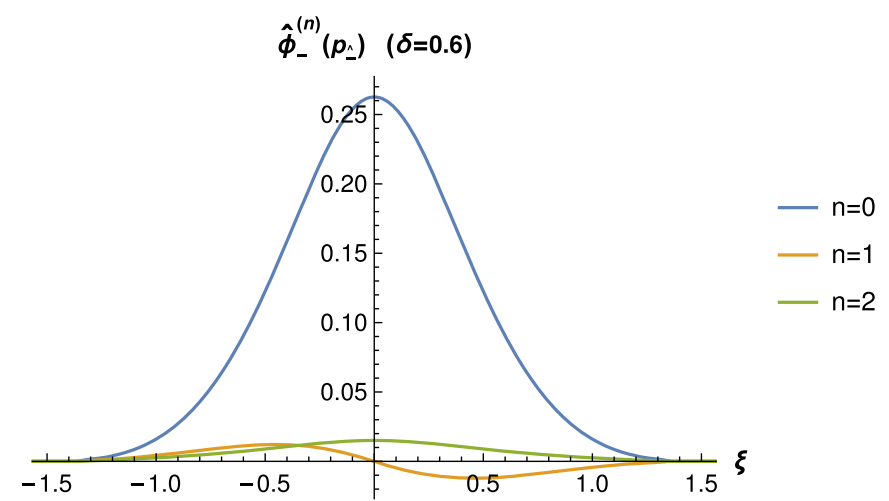

(d)

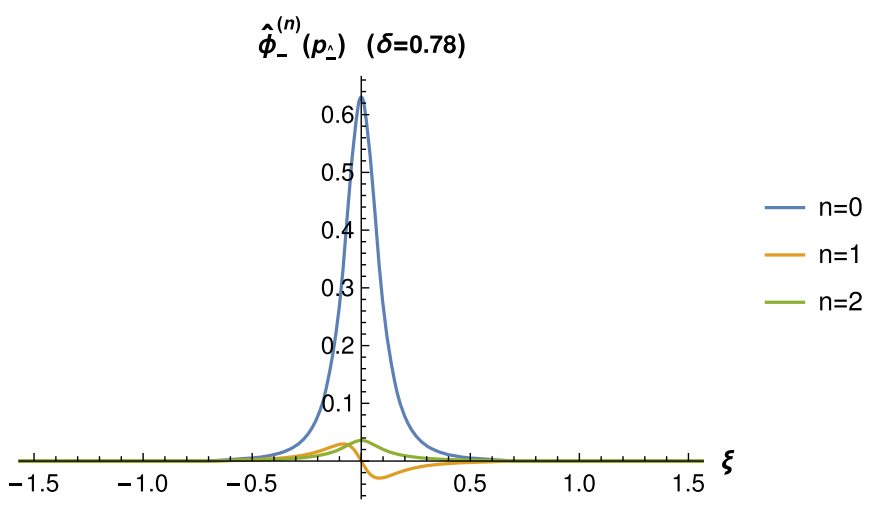

(f)

FIG. 44. Rest frame wave functions $\hat{\phi}_{+}^{(n)}\left(p_{\hat{\iota}}\right)$ and $\hat{\phi}_{-}^{(n)}\left(p_{\curlywedge}\right)$ for $m=0.18$ as functions of $\xi=\tan ^{-1} p_{\iota}$. All quantities are in proper units of $\sqrt{2 \lambda}$.

the variable of the horizontal axis $\xi=\tan ^{-1}\left(p_{\wedge}\right)$. In Fig. 41, the interpolation angle $\delta=0,0.6$, and 0.78 results are depicted in solid, dashed, and dotted lines, respectively.

In contrast to the ground state, the excited states $(n \neq 0)$ for $m=0$ acquire the nonzero bound-state mass as shown in Table VI, e.g., $\mathcal{M}_{(1)}=2.43, \mathcal{M}_{(2)}=3.76$, etc. in the unit of $\sqrt{2 \lambda}$, and they can take the rest frame $r_{\hat{\imath}}=0$. In Fig. 42, the bound-state wave functions $\hat{\phi}_{+}^{(n)}\left(p_{\hat{\varkappa}}\right)$ and $\hat{\phi}_{-}^{(n)}\left(p_{\lrcorner}\right)$for the first $(n=1)$ and second $(n=2)$ excited states obtained by numerically solving Eq. (F1) are plotted with yellow and green lines, respectively. From Fig. 42, we can see the odd and even parities respectively for $n=1$ and $n=2$ state wave functions $\hat{\phi}_{ \pm}^{(n)}\left(p_{\hat{\iota}}\right)$ under the exchange of $p_{\iota} \leftrightarrow-p_{\iota}$. For the $\delta$ value close to $\pi / 4$, the wave functions are very sharply peaked and constrained in a relatively small $\left|p_{\hat{A}}\right|$ region, indicating that ultimately only the zero-mode $p^{+}=0$ survives for $r^{+}=0$ frame in LFD. Not only does the supporting momentum region 


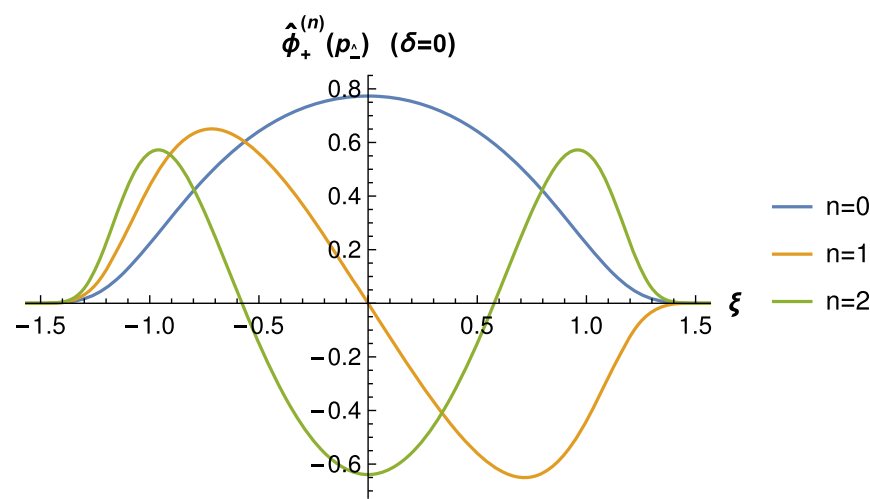

(a)

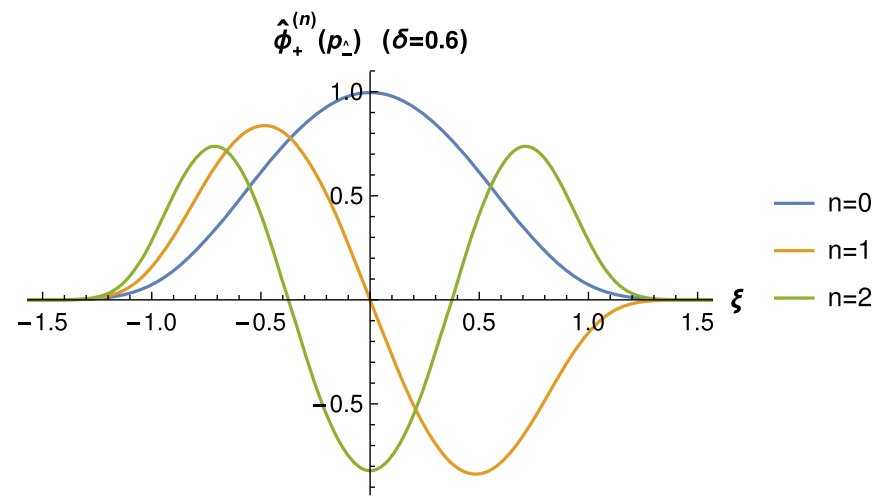

(c)

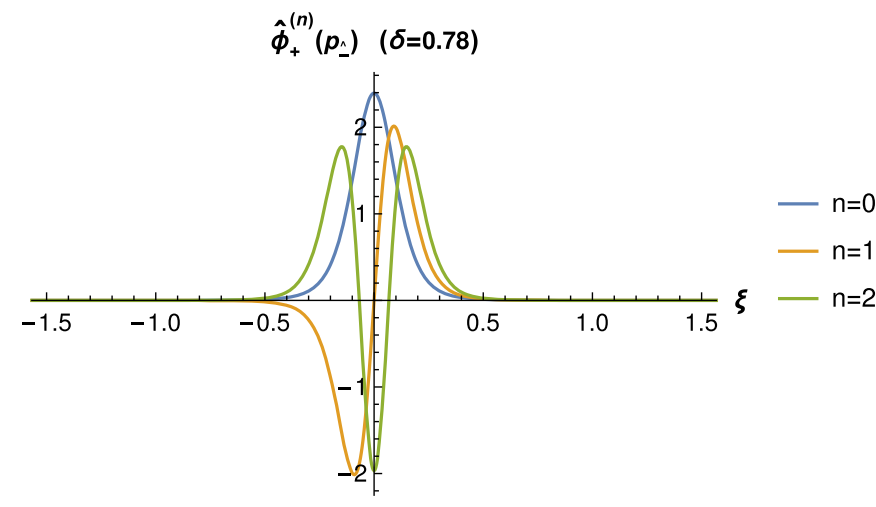

(e)

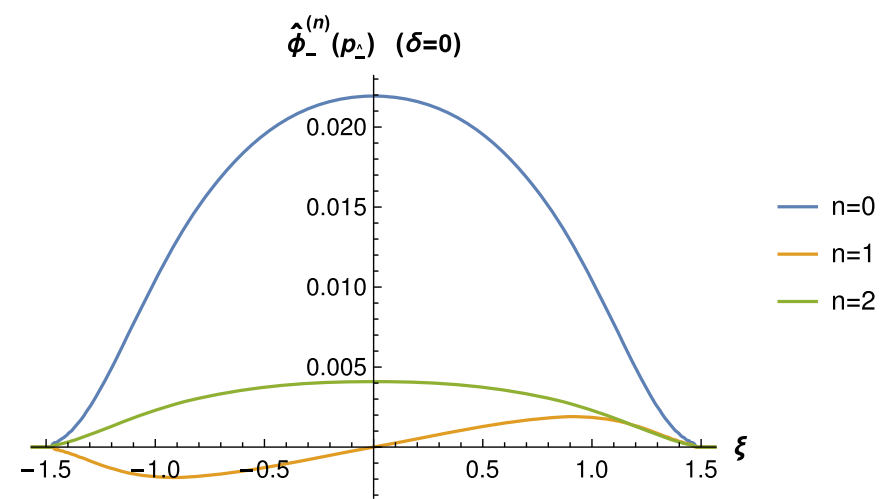

(b)

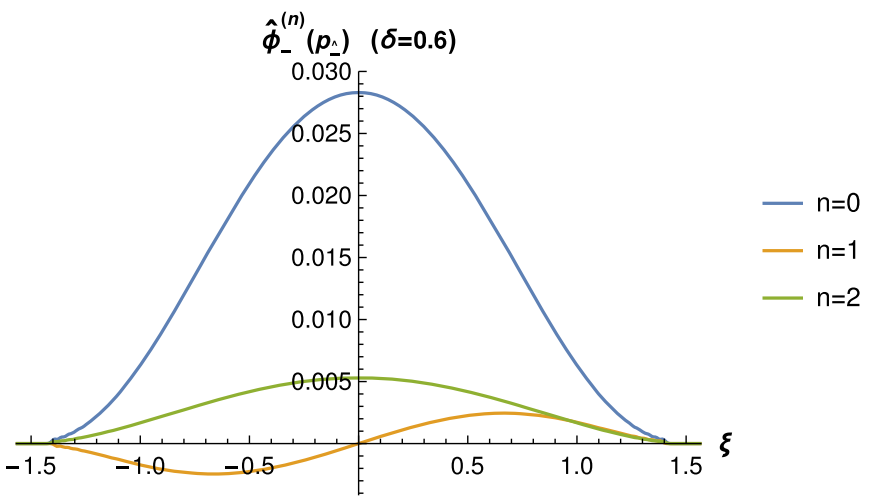

(d)

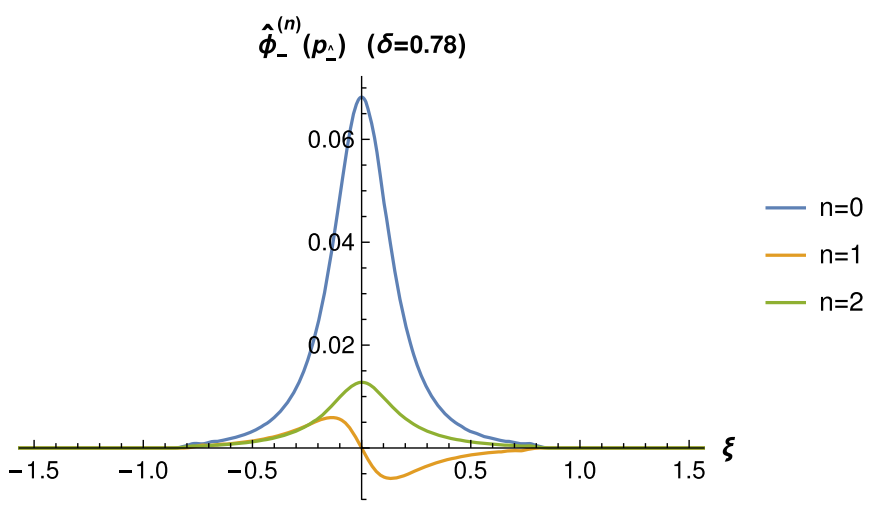

(f)

FIG. 45. Rest frame wave functions $\hat{\phi}_{+}^{(n)}\left(p_{\lrcorner}\right)$and $\hat{\phi}_{-}^{(n)}\left(p_{\lrcorner}\right)$for $m=1.0$ as functions of $\xi=\tan ^{-1} p_{\lrcorner}$. All quantities are in proper units of $\sqrt{2 \lambda}$.

$\xi=\tan ^{-1}\left(p_{\hat{\iota}}\right)$ for $\hat{\phi}_{ \pm}^{(n)}\left(p_{\hat{\iota}}\right)$ get shrunken to the zero-mode $\xi=\tan ^{-1}\left(p^{+}\right)=0$ but also the magnitude of $\hat{\phi}_{-}^{(n)}\left(p_{\hat{\varkappa}}\right)$ gets much more suppressed compared to $\hat{\phi}_{+}^{(n)}\left(p_{\hat{\Lambda}}\right)$ as $\delta$ value gets close to $\pi / 4$, indicating the absence of $\hat{\phi}_{-}^{(n)}\left(p^{+}\right)$ solutions in LFD.

For the nonzero mass cases, we plot $n=0,1$, and 2 together for the rest frame wave functions $\hat{\phi}_{+}^{(n)}\left(p_{\hat{\iota}}\right)$ and $\hat{\phi}_{-}^{(n)}\left(p_{\lrcorner}\right)$as shown in Fig. 43 for $m=0.045$, Fig. 44 for $m=0.18$, Fig. 45 for $m=1.0$, and Fig. 46 for $m=2.11$, respectively.
For $m=0.045$, although we have compared our results for the moving frames shown in Figs. 29 and 30 with the corresponding results in Ref. [12] as discussed in Appendix D, we could not compare our results shown in Fig. 43 with Ref. [12] as the rest frame was not considered in Ref. [12]. However, the results shown in Fig. 43 are not much different from the corresponding results for $m=0$ discussed above as the difference in the mass $m$ is rather marginal.

For $m=0.18,1.0$ and 2.11, all of our $\delta=0$ results shown in Figs. 44, 45, and 46 can be respectively compared 


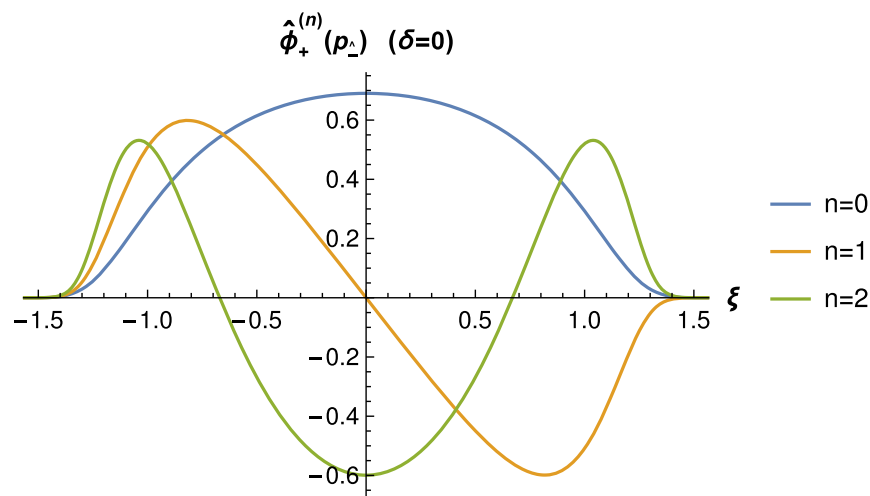

(a)

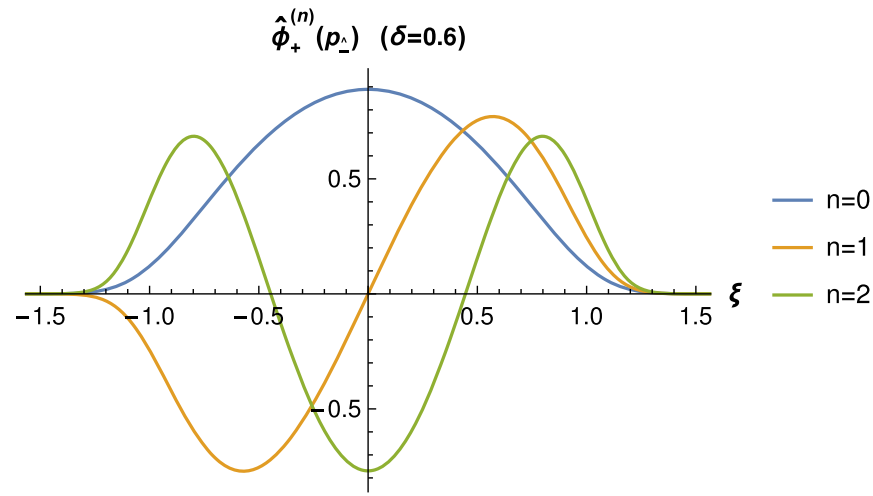

(c)

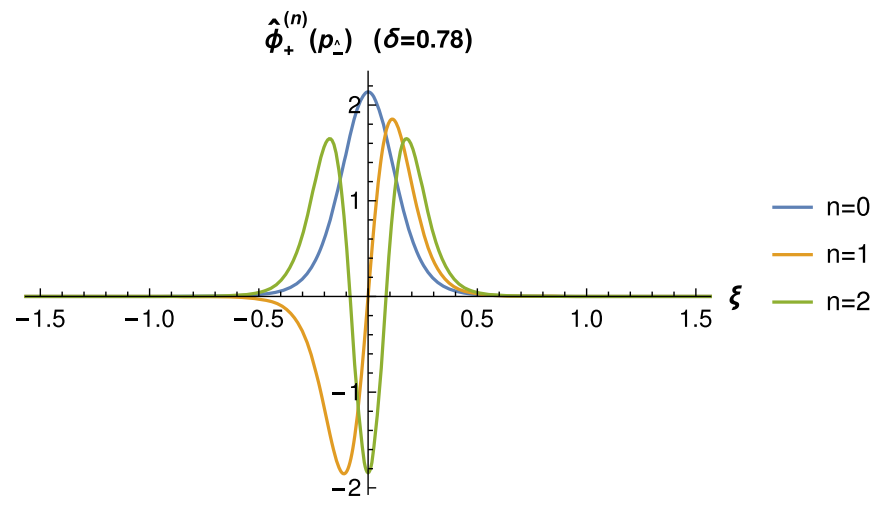

(e)

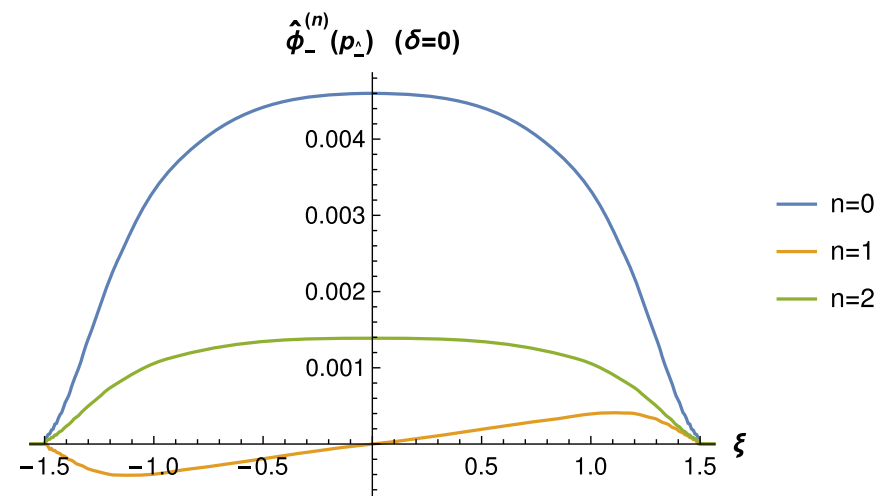

(b)

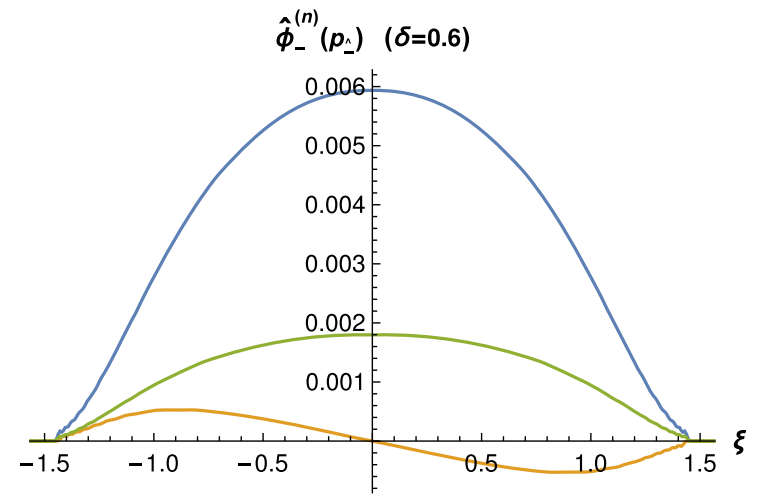

(d)

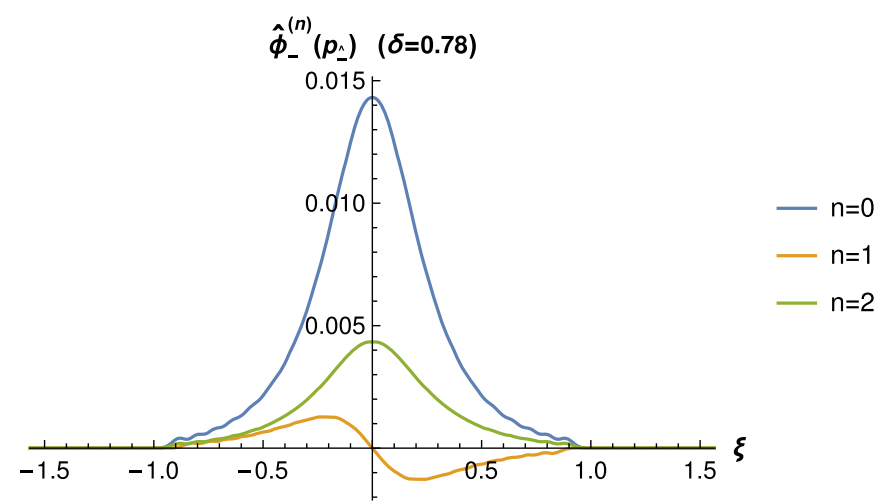

(f)

FIG. 46. Rest frame wave functions $\hat{\phi}_{+}^{(n)}\left(p_{\hat{\iota}}\right)$ and $\hat{\phi}_{-}^{(n)}\left(p_{\hat{\iota}}\right)$ for $m=2.11$ as functions of $\xi=\tan ^{-1} p_{\hat{\iota}}$. All quantities are in proper units of $\sqrt{2 \lambda}$.

with the corresponding rest frame results shown in Figs. 7, 8, 9 and 10 of Ref. [11]. Although the normalization of the bound-state wave function was mistaken in Ref. [11] by taking the + sign between $\left|\hat{\phi}_{+}^{(n)}\left(p_{\hat{A}}\right)\right|^{2}$ and $\left|\hat{\phi}_{-}^{(n)}\left(p_{\hat{A}}\right)\right|^{2}$ in Eq. (F5), our results look quite consistent with theirs as the magnitude of $\hat{\phi}_{+}^{(n)}\left(p_{\hat{\iota}}\right)$ is much larger than the magnitude of $\hat{\phi}_{-}^{(n)}\left(p_{\hat{A}}\right)$ to reveal any sizable difference for the comparison. All of our results look consistent with the characteristics of $\hat{\phi}_{+}^{(n)}\left(p_{\hat{\iota}}\right)$ and $\hat{\phi}_{-}^{(n)}\left(p_{\hat{\iota}}\right)$ discussed previously. 
[1] G. 't Hooft, Nucl. Phys. B75, 461 (1974).

[2] G. 't Hooft, Nucl. Phys. B72, 461 (1974).

[3] E. Witten, Nucl. Phys. B160, 57 (1979).

[4] A. R. Zhitnitsky, Phys. Lett. 165B, 405 (1985); 44, 139 (1986); Phys. Rev. D 53, 5821 (1996).

[5] V. Baluni, Phys. Lett. 90B, 407 (1980).

[6] G. Bhattacharya, Nucl. Phys. B205, 461 (1982).

[7] P. Steinhardt, Nucl. Phys. B176, 100 (1980).

[8] C. G. Callan, N. Coote, and D. J. Gross, Phys. Rev. D 13, 1649 (1976).

[9] Y. Frishman, C. T. Sachrajda, H. Abarbanel, and R. Blankenbecler, Phys. Rev. D 15, 2275 (1977).

[10] I. Bars and M. B. Green, Phys. Rev. D 17, 537 (1978).

[11] M. Li, L. Wilets, and M. C. Birse, J. Phys. G 13, 915 (1987).

[12] Y. Jia, S. Liang, L. Li, and X. Xiong, J. High Energy Phys. 11 (2017) 151.

[13] K. Hornbostel, Phys. Rev. D 45, 3781 (1992).

[14] P. A. M. Dirac, Rev. Mod. Phys. 21, 392 (1949).

[15] C.-R. Ji, Z. Li, B. Ma, and A. T. Suzuki, Phys. Rev. D 98, 036017 (2018).

[16] C.-R. Ji and C. Mitchell, Phys. Rev. D 64, 085013 (2001).

[17] C.-R. Ji and A. T. Suzuki, Phys. Rev. D 87, 065015 (2013).

[18] C.-R. Ji, Z. Li, and A. T. Suzuki, Phys. Rev. D 91, 065020 (2015).

[19] Z. Li, M. An, and C.-R. Ji, Phys. Rev. D 92, 105014 (2015).

[20] S. S. Chabysheva and J. R. Hiller, Phys. Rev. D 102, 116010 (2020).

[21] A. Ilderton, G. Torgrimsson, and J. Wardh, Phys. Rev. D 92 , 065001 (2015).

[22] Y. Jia, S. Liang, X. Xiong, and R. Yu, Phys. Rev. D 98, 054011 (2018).

[23] C.-R. Ji and S. Rey, Phys. Rev. D 53, 5815 (1996).

[24] M. E. Peskin and D. V. Schroeder, An Introduction to Quantum Field Theory (Addison-Wesley Publishing Company, Reading, 1997).
[25] F. Lenz, M. Thies, S. Levit, and K. Yazaki, Ann. Phys. (N.Y.) 208, 1 (1991).

[26] S. Coleman, Commun. Math. Phys. 31, 259 (1973).

[27] M. Burkardt, Phys. Rev. D 53, 933 (1996).

[28] M. Li, Phys. Rev. D 34, 3888 (1986).

[29] P. J. A. Bicudo, J. E. F. T. Ribeiro, and A. V. Nefediev, Phys. Rev. D 65, 085026 (2002).

[30] Y.S. Kalashnikova and A. V. Nefed'ev, Phys. Usp. 45, 347 (2002).

[31] C.-R. Ji, Few-Body Syst. 52, 421 (2012).

[32] T. T. Wu, Phys. Rep. 49, 245 (1979); Phys. Lett. 71B, 142 (1977).

[33] D. B. Carpenter, Nucl. Phys. B228, 365 (1983).

[34] R. C. Brower, W. L. Spence, and J. H. Weis, Phys. Rev. D 19, 3024 (1979).

[35] B. Grinstein and R. F. Lebed, Phys. Rev. D 57, 1366 (1998).

[36] M. Burkardt and N. Uraltsev, Phys. Rev. D 63, 014004 (2000).

[37] L. Ya. Glozman, V. K. Sazonov, M. Shifman, and R. F. Wagenbrunn, Phys. Rev. D 85, 094030 (2012).

[38] S. Dalley and I. R. Klebanov, Phys. Rev. D 47, 2517 (1993).

[39] J. Ellis, Acta Phys. Pol. B 8, 1019 (1977).

[40] M. M. Brisudová, L. Burakovsky, and T. Goldman, Phys. Rev. D 61, 054013 (2000).

[41] X. Ji, Phys. Rev. Lett. 110, 262002 (2013); Sci. China Phys. Mech. Astron. 57, 1407 (2014).

[42] Y. Hatta, X. Ji, and Y. Zhao, Phys. Rev. D 89, 085030 (2014).

[43] J. Collins, Foundations of Perturbative QCD (Cambridge University Press, Cambridge, England, 2013).

[44] K. Hornbostel, The application of light-cone quantization to quantum chromodynamics in one-plus-one dimensions, SLAC-333, UC-34D (T), Ph.D. dissertation, Stanford, 1988. 